Aus der Abteilung Psychosomatische Medizin und Psychotherapie

(Prof. Dr. med. C. Hermann-Lingen)

im Zentrum Psychosoziale Medizin

der Medizinischen Fakultät der Universität Göttingen

\title{
Veränderung von Problemen und Zielen im Therapieverlauf
}

Eine Untersuchung von verhaltenstherapeutisch und psychodynamisch behandelten Patienten mit Generalisierter Angststörung

$$
\begin{gathered}
\text { INAUGURAL - DISSERTATION } \\
\text { zur Erlangung des Doktorgrades } \\
\text { der Medizinischen Fakultät }
\end{gathered}
$$

der Georg-August-Universität zu Göttingen

vorgelegt von

Frauke Carola Wiegand

aus

Siegen

Göttingen 2010 
Dekan:

I.Berichterstatter:

II.Berichterstatter:

III.Berichterstatterin:

Tag der mündlichen Prüfung:
Prof. Dr. med. C. Frömmel

Prof. Dr. med. H. Staats

Prof. Dr. med. B. Bandelow

Prof. Dr. rer. nat. P. Virsik-Köpp

13.12.2010 


\section{Inhalt:}

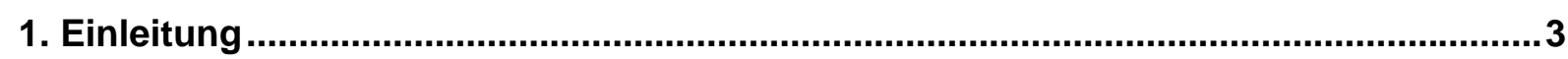

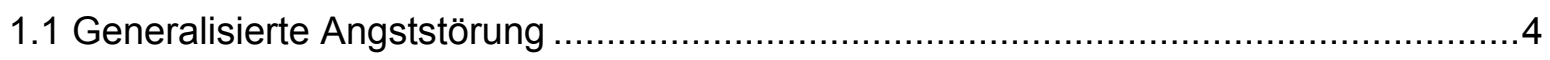

1.1.1 Definition, Epidemiologie und Therapie der Generalisierten Angststörung ..............4

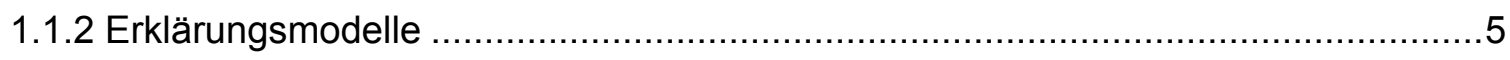

1.1.3 Darstellung der beiden in der vorliegenden Dissertation verglichenen

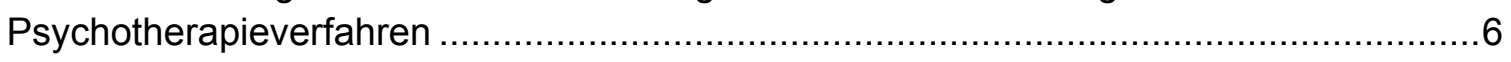

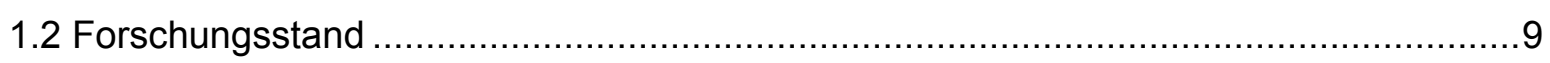

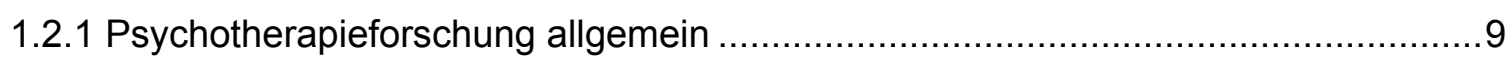

1.2.2 Psychotherapie bei Generalisierter Angststörung.......................................... 12

1.3 Zielsetzung der Studie, Fragestellung und Hypothesen .......................................13

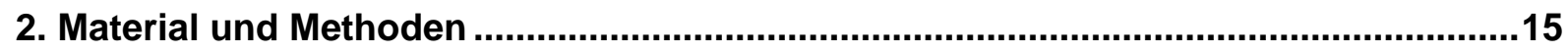

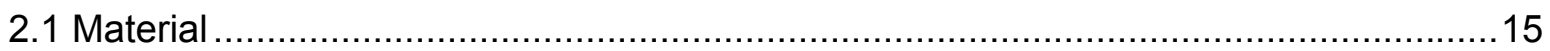

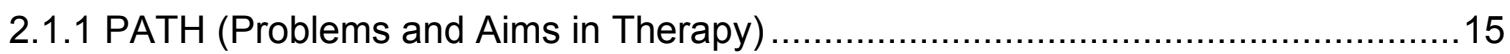

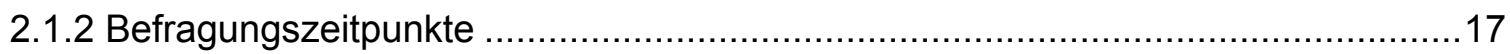

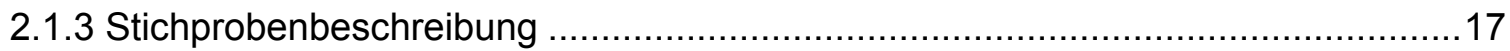

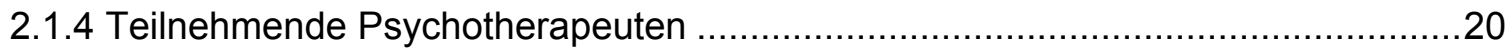

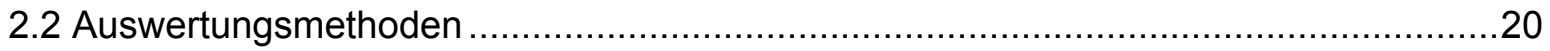

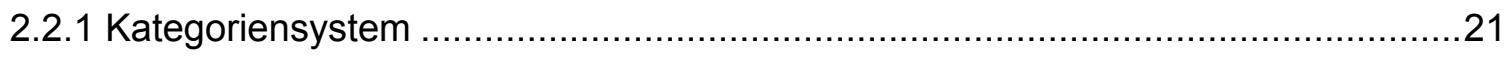

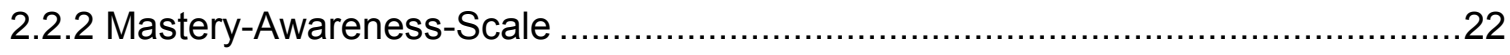

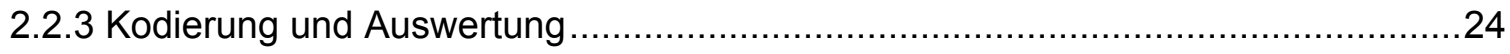

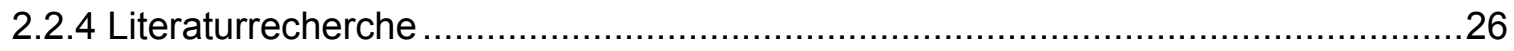

3. Darstellung der eigenen Untersuchungen, Ergebnisse …....................................27

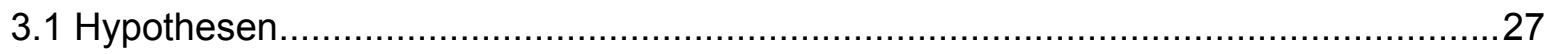

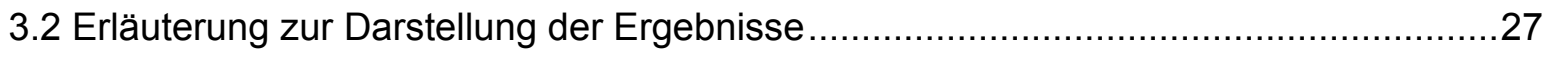

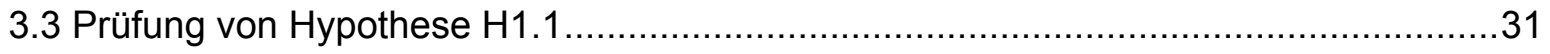

3.3.1 Verteilung von allen genannten Problemen und Zielen auf Inhaltskategorien des Kategoriensystems...

3.3.2 Verteilung von allen genannten Problemen und Zielen auf Inhaltskategorien des Kategoriensystems, Vergleich Frauen und Männer .................................................... 37

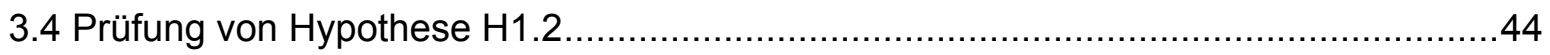

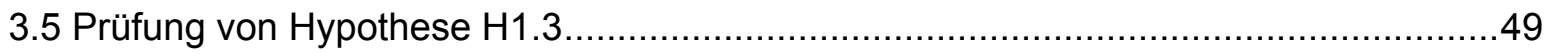

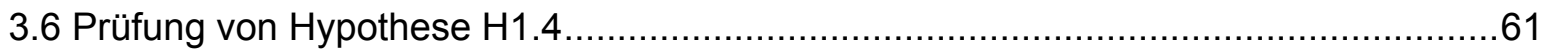

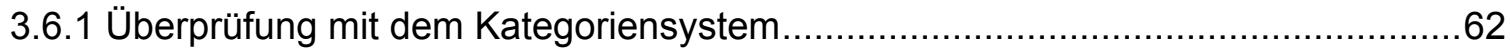

3.6.2 Überprüfung mit der Mastery-Awareness-Scale ...............................................62 
3.6.3 Prüfung der Hypothese mittels Selbsteinschätzung der Patienten bezüglich ihres Leidensdrucks unter zu Befragungszeitpunkt $t 1$ angegebenen Problemen und der

Verwirklichung zu t1 angegebener Ziele .69

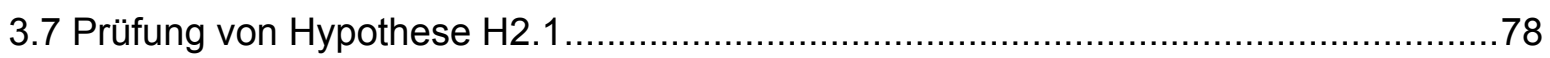

3.7.1 Allgemeine Prädiktoren verschiedener Therapieverläufe .81

3.7.2 Inhaltskategorien des Kategoriensystems als Prädiktoren verschiedener Therapieverläufe

3.7.3 Wahrnehmungs- und Bewältigungskategorien der Mastery-Awareness-Scale als

Prädiktoren verschiedener Therapieverläufe .84

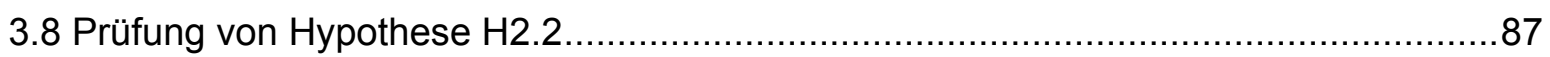

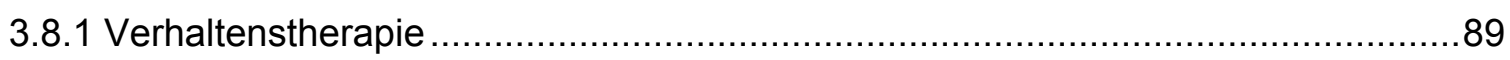

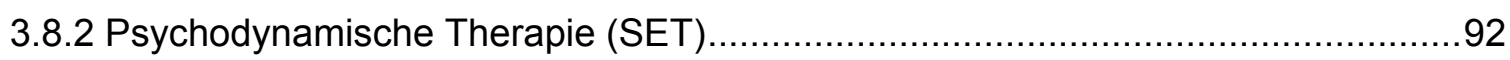

3.8.3 Vergleich der Verteilung von Prädiktoren innerhalb der Therapierichtungen VT

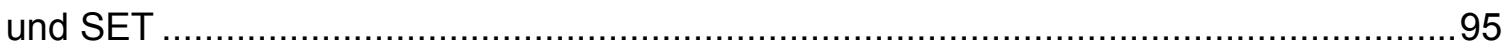

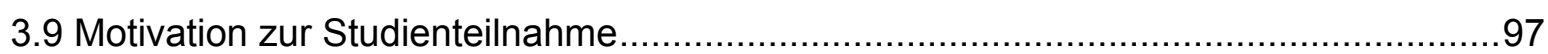

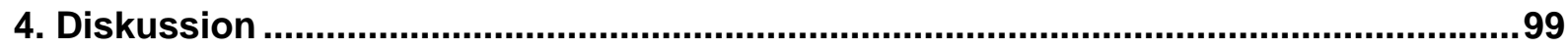

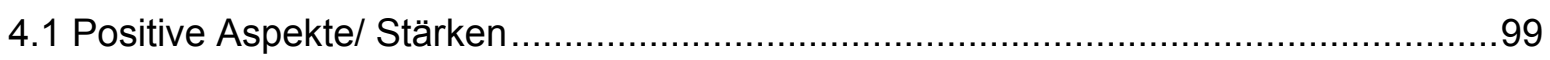

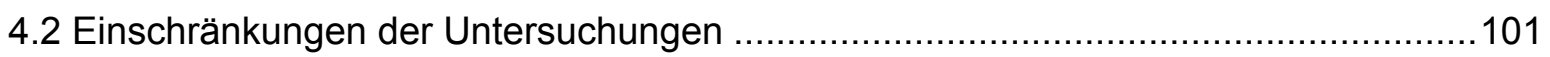

4.3 Diskussion der Ergebnisse im Einzelnen............................................................ 104

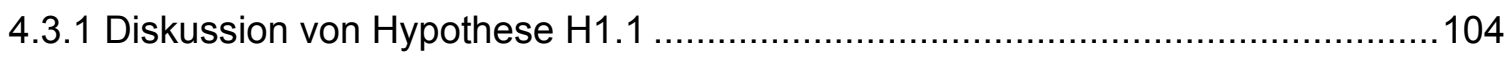

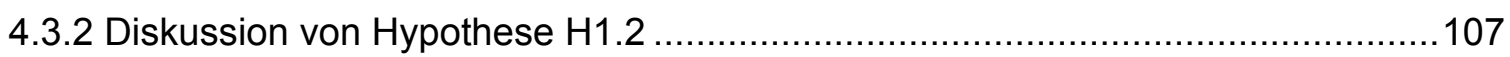

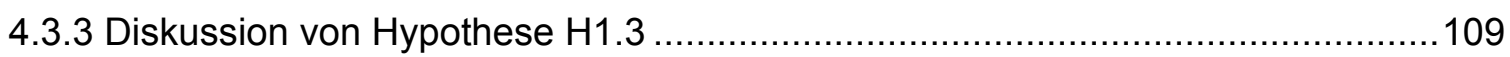

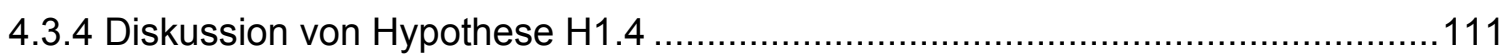

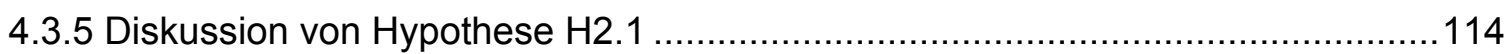

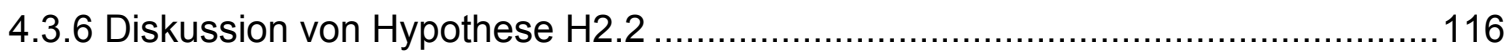

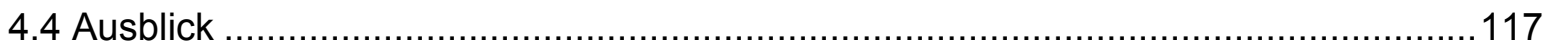

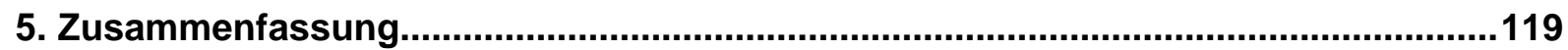

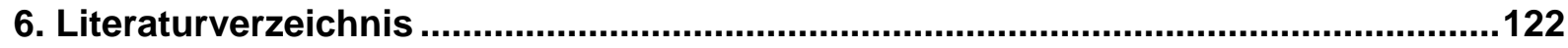

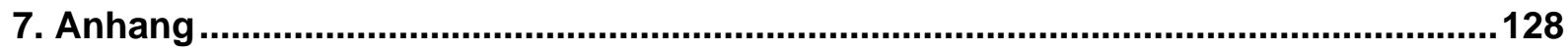

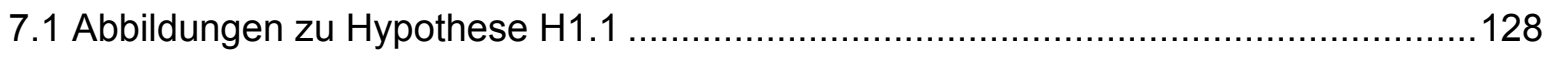

7.1.1 Problem- und Zielangaben aller Patienten, Verteilung auf Inhaltskategorien des

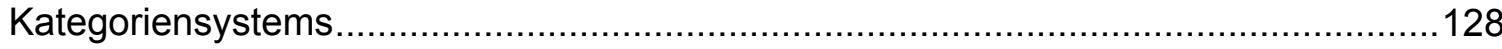

7.1.2 Problem- und Zielangaben aller Patienten, Verteilung auf Inhaltskategorien des Kategoriensystems, Vergleich Frauen und Männer .................................................. 129

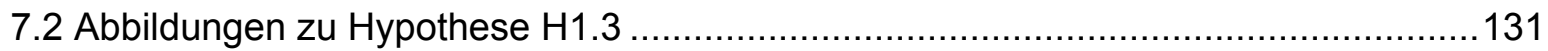

7.3 Abbildungen, Tabellen und statistische Definitionen zu Hypothese H1.4................132

7.3.1 Rating von Problemen und Zielen mit der Mastery-Awareness-Scale .................132 
7.3.2 Systematische Veränderungen der Selbsteinschätzung von Patienten/ Signifikanztests 144

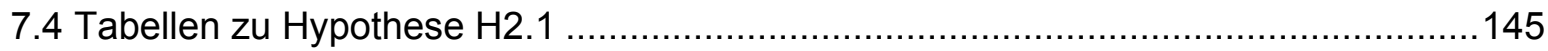

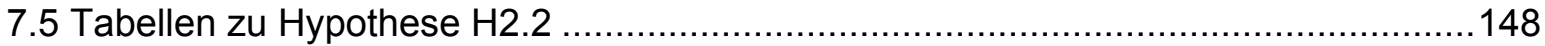




\section{Abkürzungsverzeichnis}

DSM Diagnostisches und Statistisches Manual Psychischer Störungen (Diagnostic and Statistical Manual of Mental Disorders) ist ein Klassifikationssystem psychischer Störungen der American Psychiatric Association, ähnlich dem ICD (Internationale Klassifikation der Krankheiten) der WHO (Häcker und Stapf 2004).

\section{GAS Generalisierte Angststörung}

ist eine häufige Form der Angststörung. Die in dieser Dissertation ausgewertete Studie untersucht verschiedene Therapieverfahren bei Patienten mit GAS. Nähere Angaben unter 1.1.

\section{PATH Problems and Aims in Therapy}

ist ein Instrument zur Erfassung von inhaltlichen Veränderungen in einer Psychotherapie (Staats et al. 1996). Dabei werden Probleme und Ziele der Patienten erfragt und durch diese selbst wiederholt bezüglich des Leidensdrucks und der Zielverwirklichung beurteilt. Nähere Angaben unter 2.1.1.

\section{supportiv-expressive Therapie}

ist eine von Lester Luborsky entwickelte Form der psychodynamischen Psychotherapie. In der vorliegenden Arbeit wird unter dem Begriff „psychodynamische Therapie" oder "SET" eine speziell für das Störungsbild der GAS operationalisierte Variante dieser Behandlung verstanden (vgl. Leichsenring et al. 2005). Nähere Angaben unter 1.1.3.

\section{Verhaltenstherapie}

wird hier synonym für kognitiv-behaviorale Therapie (CBT) verwendet und ist eine Psychotherapieform, die auf der Grundlage der Lerntheorie basiert (Häcker und Stapf 2004).

In der vorliegenden Arbeit wird eine für das Krankheitsbild der Generalisierten Angststörung erstellte, manualisierte Form der kognitivbehavioralen Therapie zugrunde gelegt. Nähere Angaben unter 1.1.3. 
ist eine Operationalisierung des Konzeptes der Übertragung, die in der supportiv-expressiven Therapie (SET) nach Lester Luborsky eine zentrale Bedeutung hat. Interpersonelle und intrapsychische Konflikte werden als ein zentrales Konfliktthema formuliert und sind so der weiteren Analyse zugänglich (Leichsenring et al. 2005). Nähere Angaben unter 1.1.3.

In Anbetracht besserer Lesbarkeit wurden folgende Vereinfachungen vorgenommen: Für Bezeichnungen von Personen wird (abgesehen vom Vergleich weiblicher und männlicher Studienteilnehmer) die männliche Sprachform verwendet.

Wie bereits erwähnt, werden die Begriffe psychodynamische Therapie und SET im Folgenden gleichgesetzt (siehe dazu Diskussion unter 4.2). 


\section{Einleitung}

Wird eine Erkrankung behandelt, so soll dies mit einer möglichst für das Krankheitsbild passenden und effektiven Therapie geschehen. Häufig bieten sich mehrere Behandlungsformen an, welche in ihrer Wirksamkeit und Art der Wirkung überprüft werden müssen. Im Vergleich kann ggf. herausgefunden werden, für welches Krankheitsbild und für welche Patienten das jeweilige Verfahren besonders geeignet ist.

Diese Dissertation untersucht die Kurzzeit-Psychotherapie des Störungsbildes der Generalisierten Angststörung (im Folgenden GAS). Als Teilbereich einer durch die Deutsche Forschungsgemeinschaft (DFG) geförderten, randomisierten und kontrollierten Studie wurden nach einem besonderen Verfahren (PATH, siehe 2.1.1) frei formulierte Texte von GAS-Patienten während und nach einer Kurzzeitpsychotherapie erhoben. Diese bereits vorliegenden Daten werden u. a. mit der vorliegenden Dissertation überwiegend deskriptiv ausgewertet.

Teilergebnisse der Gesamtstudie mit anderen Fragestellungen wurden bereits veröffentlicht (Leichsenring et al. 2009, Winkelbach 2007).

Diese Promotionsarbeit soll einen Beitrag zur Psychotherapie-Forschung leisten, indem zwei psychotherapeutische Behandlungsmethoden in der Behandlung von GAS näher untersucht werden. Dieses Forschungsfeld gewinnt zunehmend an Bedeutung, zumal Forschungslücken im Bereich der Therapieeffekte psychodynamischer Psychotherapie-Formen bei der Behandlung von GAS bestehen (Leichsenring et al. 2009). In dieser Arbeit wird daher erforscht, ob und wie messbar sich wichtige Ziele und Probleme im Verlauf einer Psychotherapie aus Sicht der Patienten verändern. Die nach PATH erhobenen Daten bilden dafür die Grundlage. Erstmals wird damit das PATH-Verfahren im Rahmen von Kurzzeitpsychotherapien eines bestimmten Störungsbildes angewendet und somit auch ein Beitrag zur weiteren Erprobung von PATH geleistet. Die Probleme und Ziele werden inhaltlich analysiert und hinsichtlich der Problemwahrnehmung und -bewältigung der Patienten beurteilt. Zentral ist hierbei auch die Frage, wie diese Entwicklung unter den zwei untersuchten Psychotherapieformen ist. Schließlich können Änderungen der Probleme und Ziele sowie deren Wahrnehmung und Bewältigung einen therapeutischen Prozess anzeigen und Anlass zu weiteren (Forschungs-)Überlegungen bieten. 


\subsection{Generalisierte Angststörung}

Allen 51 Patienten, deren Daten in dieser Arbeit ausgewertet werden, ist gemeinsam, dass bei innen eine GAS diagnostiziert wurde. Im Folgenden wird daher dieses Störungsbild genauer vorgestellt.

\subsubsection{Definition, Epidemiologie und Therapie der Generalisierten Angststörung} Nach ICD-10 ${ }^{1}$ Definition wird unter der Generalisierten Angststörung Folgendes verstanden (F41.1, DIMDI 2008, S. 185): „Die Angst ist generalisiert und anhaltend. Sie ist nicht auf bestimmte Umgebungsbedingungen beschränkt, oder auch nur besonders betont in solchen Situationen, sie ist vielmehr "frei flottierend“. Die wesentlichen Symptome sind variabel, Beschwerden wie ständige Nervosität, Zittern, Muskelspannung, Schwitzen, Benommenheit, Herzklopfen, Schwindelgefühle oder Oberbauchschmerzen gehören zu diesem Bild. Häufig wird die Befürchtung geäußert, der Patient selbst oder ein Angehöriger könnten demnächst erkranken oder einen Unfall haben."

Die Definition nach DSM-IV² betont stattdessen stärker das Sich-Sorgen im Sinne einer "furchtsamen Erwartung" mit "Schwierigkeiten, die Sorgen zu stoppen“ (Saß et al. 2003, S. 525). Das klinische Bild muss definitionsgemäß über mindestens sechs Monate an den meisten Tagen vorhanden sein, um die Kriterien einer GAS zu erfüllen.

Nach Leibing et al. (2003) wurde GAS erstmalig 1980 als eigenes Krankheitsbild dokumentiert und ist nach der Depression die am zweithäufigsten vorkommende psychische Störung. Die Lebenszeitprävalenz beträgt 4-5\%, in der Praxis treten oftmals Komorbiditäten mit anderen psychischen Erkrankungen auf, wobei vor allem Depression und andere Angststörungen zu nennen sind. Häufig zeigt sich eine Chronifizierung.

Aufgrund bisheriger Untersuchungen ist davon auszugehen, dass Frauen öfters betroffen sind als Männer. Der Anteil von GAS-Patientinnen im Rahmen epidemiologischer Studien liegt demnach bei ca. zwei Dritteln (Saß et al. 2003). Allerdings liegen

\footnotetext{
${ }^{1}$ ICD $10=$ Internationale statistische Klassifikation der Krankheiten und verwandter Gesundheitsprobleme, 10. Revision (DIMDI 2008)

${ }^{2}$ DSM IV = Diagnostisches und Statistisches Manual Psychischer Störungen, Version IV (Saß et al. 2003)
} 
zur Fragestellung der Geschlechterverteilung bisher nur wenig verlässliche Daten vor (Becker und Margraf 2007).

Außerdem weisen neuere Studien darauf hin, dass das Störungsbild der GAS zunehmend häufiger auftritt (Mecker und Margraf 2007).

Meist findet sich bei GAS-Patienten ein deutliches Vermeidungsverhalten gegen ängstigende Situationen, entweder offen oder verdeckt (Leibing et al. 2003). Oftmals beginnt die Symptomatik der GAS bereits im Kindesalter oder der Adoleszenz, allerdings kann die Erkrankung auch nach dem 20. Lebensjahr auftreten (Saß et al. 2003). Differentialdiagnostisch abzugrenzen sind Diagnosen wie Zwangsstörung (F42), depressive Episode (F32), phobische Störung (F40) oder Panikstörung (F42).

Darüber hinaus sind substanzinduzierte oder in medizinischen Ursachen begründete Angststörungen sowie posttraumatische Belastungsstörungen (F43.1) oder Anpassungsstörungen (F43.2) von einer GAS zu unterscheiden (vgl. Saß et al. 2003). Saß et al. grenzen die GAS folgendermaßen von Angst ohne Krankheitswert ab: GASPatienten haben Schwierigkeiten, die mit der Krankheit einhergehenden Sorgen zu kontrollieren und werden dadurch funktionell eingeschränkt. Die Sorgen im Rahmen einer GAS sind insgesamt schwerwiegender als Sorgen ohne pathologische Bedeutung, oftmals treten sie ohne konkreten Auslöser auf. Zudem zeigen sich bei einer GAS zusätzlich körperliche Begleitsymptome, welche bei „normalen“ Sorgen kaum vorkommen. Insgesamt sind die GAS-Sorgen in Dauerhaftigkeit, Ausmaß und Häufigkeit im Vergleich zu nicht pathologischen Sorgen klar überzogen.

Gemäß Leichsenring et al. (2005) wird ungefähr die Hälfte der GAS-Patienten medikamentös behandelt (z.B. durch Antidepressiva oder Benzodiazepine). Allerdings kommt es unter diesen Therapien häufig zu Rückfällen sowie Mediakamentenabhängigkeit. Als Alternative oder in Kombination steht die psychotherapeutische Behandlung zur Verfügung, hier sind Verfahren wie kognitive Verhaltenstherapie oder psychodynamische Psychotherapie zu nennen, welche im Rahmen dieser Dissertation untersucht und unter 1.1.3 dargestellt werden.

\subsubsection{Erklärungsmodelle}

Ätiologisch existiert kein einheitliches Erklärungsmodell der GAS. Es wird vielmehr von einem multifaktoriellen Geschehen ausgegangen, wobei genetische, neurobiologische und psychologische Vulnerabilitäten entscheidend zu sein scheinen. So spre- 
chen beispielsweise Zwillingsstudien für eine genetische Disposition der Entstehung dieser Störung (Saß et al. 2003).

Darüber hinaus existieren in beiden mit der vorliegenden Arbeit verglichenen Psychotherapieformen (siehe 1.1.3) eigene Störungsmodelle der Pathogenese einer GAS, woraus sich wiederum Schwerpunkte der jeweiligen Behandlung ableiten lassen. Nach Senf und Broda (2005) werden diese Modelle im Folgenden dargestellt:

\section{Kognitiv-behaviorales Erklärungsmodell der GAS:}

Der verhaltenstherapeutische Erklärungsentwurf teilt Sorgen, welche bei GASPatienten auftreten, in zwei unterschiedliche Kategorien ein. „Typ-1-Sorgen“ beschäftigen sich mit alltäglichen, wie beispielsweise finanziellen oder beruflichen Fragestellungen. „Typ-2-Sorgen“ („Meta-Sorgen“) beinhalten hingegen das eigene SichSorgen und charakterisieren dieses als krankheitsbringend. Diese Art von Besorgnis bedingt damit maßgeblich den Leidensdruck der Patienten.

Die Aufrechterhaltung der Meta-Sorgen geschieht durch verschiedene Mechanismen. Zum einen sind die Patienten besonders empfindsam für schlechte Neuigkeiten, sei es im privaten Umfeld oder z.B. in den Medien. Dies führt auf der anderen Seite zur angstvollen Erwartung negativer Ereignisse, was wiederum die Empfindsamkeit für derartige Informationen erhöht.

\section{Psychodynamisches Erklärungsmodell der GAS:}

Die psychodynamische Erklärung der GAS beinhaltet eine Schwäche der IchStruktur. Demnach besteht ein Unvermögen, Angst durch bestimmte Abwehrmechanismen zu kontrollieren. Ätiologisch lassen sich in der Kindheit von GAS-Patienten oftmals traumatisierende Erfahrungen wie z.B. unsichere Bindungserlebnisse eruieren. Diese führen dazu, dass die Patienten möglicherweise weniger beständige Eigen- oder Fremdrepräsentanzen verinnerlichen können, so dass sie sich über eine Bezugnahme auf diese verinnerlichten Repräsentanzen vergleichsweise schwerer selbst steuern und weniger leicht Sicherheit verschaffen können.

\subsubsection{Darstellung der beiden in der vorliegenden Dissertation verglichenen Psychotherapieverfahren}

Die vorliegende Dissertation untersucht Problem- und Zielangaben von an GAS erkrankten Patienten, welche eine verhaltenstherapeutische oder psychodynamische 
Kurzzeitpsychotherapie erhielten. Kurzzeittherapie bedeutet dabei, dass sowohl unter VT, als auch unter psychodynamischer Psychotherapie (SET) bis zu 30 Behandlungssitzungen (meist fünf probatorische und 25 therapeutische Sitzungen mit jeweils 50 minütiger Länge, in der Regel wöchentlich) durchgeführt wurden.

Beiden Therapieformen ist laut Leichsenring et al. (2009) gemeinsam, dass die GASPatienten darin bestärkt werden, sich Angst auslösenden Situationen zu nähern, allerdings mit verschiedener Begründung. Bei VT sollen das Vermeiden und der Rückzug verändert werden, während SET auf eine Veränderung zentraler Beziehungskonflikte abzielt.

Es werden an dieser Stelle beide Therapieformen kurz vorgestellt. Da sie vielseitige Ausprägungen aufweisen, wird in diesem Zusammenhang das spezielle Vorgehen bei GAS erläutert.

\section{Verhaltenstherapie (VT):}

VT orientiert sich an beobachtbarem Verhalten, Lernen und an Kognitionen (Vorstellungen) der Patienten. Dabei liegt nach Wittchen und Hoyer (2006, S. 420) folgender Ansatz zu Grunde: „In der Verhaltenstherapie wird es dem Therapeuten zunächst mit bestimmten Übungen und Aufgaben sowie Befragungen darum gehen, dem Patienten zu verdeutlichen, wie bestimmte problematische Verhaltensweisen, Gewohnheiten, Denkstile und Ereignisse das Verhalten, das Denken, das Fühlen und den Körper beeinflussen und möglicherweise psychische Störungen bedingen. Er wird mit dem Patienten in der Regel dann entsprechend abgestimmte gedankliche (die Fachbezeichnung heißt: kognitive) sowie praktische Verhaltensübungen ableiten, um Veränderung zu bewirken."

Auch innerhalb der VT existieren verschiedene Verfahren, wie z.B. kognitive Therapie. Die bekanntesten Behandlungsmethoden sind dabei Exposition, Entspannungsübungen und kognitive Umstrukturierung.

Für die Kurzzeit-VT der GAS wurde ein Manual verfasst (Leibing et al. 2003), welches Besonderheiten bei der Therapie dieses Krankheitsbildes mit dem Leitsymptom des Sich-Sorgens hervorhebt. Der folgende Absatz beinhaltet Aussagen dieser Veröffentlichung: Als zentrale Begriffe für das Verständnis der GAS sind Vulnerabilität und vegetative Hyperreagibilität zu nennen. Die Verschiebung der Aufmerksamkeit hin zu "anxiogener Kognition“ (Leibing et al. 2003, S. 520) wird dabei als eine Art Denkfehler verstanden, es entsteht oftmals ein Teufelskreis der Angst, indem es zum 
Sorgen über eigentliche Sorgengedanken kommt. In der verhaltenstherapeutischen Behandlung finden daher folgende Inhalte Anwendung: Es werden Entspannungsübungen mit dem Patienten eingeübt. Darüber hinaus werden Störungsmodelle entwickelt (z.B. gedankliche Automatismen bestimmt), um „die Identifikation und Modifikation von dysfunktionalen Kognitionen und Einstellungen" (Leibing et al. 2003, S. 524) zu erreichen. Sorgen werden als "ineffektiver Versuch der Problemlösung“ (Leibing et al. 2003, S. 525) verstanden. Während einer Exposition mit ängstigenden Auslösern soll der Patient sich seine Sorgen-Inhalte möglichst realistisch vorstellen, um sie im Sinne einer Habituation verarbeiten zu können. Leibing et al. beschreiben diese Methode als eine Form der Desensibilisierung. Des Weiteren wird dem Patienten empfohlen, ein Angst-Tagebuch zu führen und es werden als Hausaufgaben bestimmte Übungen verabredet. Sind all diese Therapieschritte erfolgt, sollen mit dem GAS-Patienten Bewältigungsmöglichkeiten und eine Rückfallprophylaxe erarbeitet werden. Supportiv können sportliche Maßnahmen den Therapieverlauf günstig beeinflussen, da so versucht wird, die gesteigerte Reagibilität zu reduzieren.

\section{Psychodynamische Therapie/ supportiv-expressive Therapie (SET):}

„Im internationalen Sprachgebrauch werden alle psychoanalytisch basierten Therapien als "psychodynamische Psychotherapie“ zusammengefasst.“ (Rudolf 2006, S. 110). Damit geht diese Therapieform von Grundkonzepten der Psychoanalyse nach Freud aus, in welcher seelische Störungen mit inneren Konflikten (Wunsch und Abwehr) und einem dynamischen Unbewussten (Laplanche und Pontalis 1973) erklärt werden. Dabei spielen Erfahrungen in den frühen Beziehungen zwischen Selbst und Objekt eine wichtige Rolle. Das therapeutische Vorgehen ist an Übertragung und Gegenübertragung orientiert. Der Begriff der Übertragung wird dabei verstanden als "Wiederholung der Vergangenheit in Beziehungen der Gegenwart" (Staats 2004, S. 16). „In psychoanalytischen Behandlungen werden Übertragungen explizit gefördert, um sie dann ansprechen und verändern zu können - sie werden gedeutet." (Staats 2004, S. 14).

Die 25 psychodynamisch behandelten GAS-Patienten der hier ausgewerteten Studie wurden mit einem speziellen Verfahren therapiert. Es handelt sich dabei um eine Modifikation des SET-Konzeptes nach Lester Luborsky. Dieses wird im Folgenden nach Leichsenring et al. (2005) erläutert: 
Basierend auf einer Abwandlung von Luborskys SET-Konzept durch Crits-Christoph et al. (1995) verfassten Leichsenring et al. ein Manual für die Behandlung von GASPatienten mit SET bei einer Höchstzahl von 25 Therapiesitzungen (Kurzzeitpsychotherapie). Dabei wird von folgendem Störungsmodell ausgegangen: „Psychischen Symptomen liegen intrapsychische und interpersonelle Konflikte („zentrale Beziehungskonflikte“) zugrunde. Zentrale Beziehungskonflikte werden in Form des zentralen Beziehungskonflikt-Themas (ZBKT) formuliert. Ein ZBKT umfasst drei Komponenten: einen Wunsch (W), eine Reaktion der Objekte auf diesen Wunsch (RO), eine Reaktion des Selbst auf die Reaktion der Objekte (RS).“ (Leichsenring et al. 2005, S. 259). Das SET-Verfahren basiert somit auf der Identifizierung eines ZBKT, welches im Rahmen der Therapie genauer analysiert wird. Dabei werden expressive (deutende) und supportive (unterstützende) Techniken verwendet. Beispielsweise wird eine helfende Beziehung zwischen Patient und Therapeut innerhalb der Behandlung etabliert, in der dem Patienten Akzeptanz, Einfühlungsvermögen und Aufmunterung entgegen gebracht werden. Anders als in der klassischen Psychoanalyse wird der Therapeut angehalten, sich seinem Patienten gegenüber nicht zu „neutral“ (Leichsenring et al. 2005, S. 262) zu verhalten. Dies scheint ein speziell für GAS-Patienten besonders passender Aspekt zu sein. Widerstand und Abwehr werden nur angesprochen, wenn dies für eine Verbesserung des zentralen Beziehungskonfliktes eines Patienten erforderlich ist. Umfangreiche Veränderungen der Persönlichkeit werden nicht beabsichtigt, vielmehr soll der Patient durch die genannten Interventionen lernen, seine Krankheitssymptome als Teil der Lösung des ZBKT zu begreifen und andere Lösungsmöglichkeiten zu erproben. Ausdrücklich wird darauf hingewiesen, dass sich Ziele im Prozess der SET-Therapie verändern können.

\subsection{Forschungsstand}

\subsubsection{Psychotherapieforschung allgemein}

Nach Rudolf (2006) kommt es derzeitig im Bereich von Psychotherapien insgesamt $\mathrm{zu}$, ,...) Entwicklungen auf mehreren Ebenen:

- Im therapeutischen Umgang mit spezifischen Störungen werden behandlungspraktische Modifikationen erprobt.

- Die gewonnenen therapeutischen Erfahrungen führen zur Weiterentwicklung der Störungskonzepte (z.B. bezüglich Persönlichkeitsstörungen). 
- Die Konzepte begründen die Entwicklung von diagnostischen und therapeutischen Vorgehensweisen.

- Die solchermaßen modifizierten diagnostischen und therapeutischen Verfahren können auf ihre Effektivität und Effizienz hin wissenschaftlich überprüft werden.

- Die konzeptuell begründeten und wissenschaftlich evaluierten Therapieansätze können in standardisierter Form (manualisiert) niedergelegt und in Fort- und Weiterbildungen gelehrt und supervidiert werden.

Entwicklungen dieser Art vollziehen sich notwendigerweise angesichts neuer Aufgaben - an neuen Störungsbildern, unter veränderten gesellschaftlichen Bedingungen, auch angesichts sich wandelnder Bedingungen der Krankenversorgung und des Gesundheitssystems unter dem Einfluss der Forderung nach Ergebnistransparenz und Qualitätssicherung." (Rudolf 2006, S. 110).

Die Effektivität von Psychotherapie zu untersuchen ist dabei komplexer, als beispielsweise die Wirksamkeit bestimmter Pharmaka zu überprüfen. Hier die geeigneten Instrumente zu finden ist daher von großer Wichtigkeit.

Bei der Effektivitätsuntersuchung psychodynamischer Therapie ist laut Milrod (2009) besonders zu berücksichtigen, dass bisherige Schwierigkeiten auch darin lagen, Therapien, welche als psychodynamisch beschrieben werden, zu standardisieren. Dennoch wurden durch randomisierte, kontrollierte Studien bereits verschiedene Formen psychodynamischer Therapie (wie Psychoanalyse versus tiefenpsychologisch fundierte Psychotherapie) miteinander verglichen (Grande et al. 2006). Beutel führt ferner zur psychodynamischen Kurzzeittherapie aus: „Forschungsbedarf besteht weiterhin zur Indikation, zum Stellenwert von Kurzzeitbehandlungen im Verlauf der Behandlungskarriere und zu geeigneten Formen der Ausbildung." (Beutel 2000, S. 203).

In Deutschland hat der Wissenschaftliche Beirat Psychotherapie (WBP) 2007 erstmals Kriterien zur Anerkennung psychotherapeutischer Verfahren festgelegt (WBP 2009). Danach sollen nicht nur neue Therapien (wie z.B. Gesprächspsychotherapie), sondern auch bisher nach dem Psychotherapeutengesetz anerkannte Verfahren ihre Wirksamkeit in Bezug auf die häufigsten Störungsbilder nachweisen. Dies bezieht sich beispielsweise auf Angst- und Zwangsstörungen als oft auftretende seelische Erkrankungen. Für Art und Anzahl der zu berücksichtigenden Studien wurden genaue Kriterien festgelegt. 
Forschungsbedarf besteht zudem in der Untersuchung der Wirksamkeit psychodynamischer Verfahren in störungsspezifischen Studien sowie hinsichtlich längerer Nachbeobachtungszeiten bei Studien bezüglich der Wirksamkeit von VT. Für beide Therapieverfahren soll diese Forschungslücke durch die vorliegende, störungsspezifische (GAS) Studie mit einjähriger Nachbeobachtung geschlossen werden.

Der Vergleich von psychoanalytischer Psychotherapie und VT wird schon seit einiger Zeit kontrovers diskutiert. In einer Meta-Analyse hat Grawe bereits 1994 psychodynamische und verhaltenstherapeutische Behandlungen sowohl in Langzeit-, als auch in Kurzzeittherapien verglichen und kam zu dem Ergebnis, dass psychoanalytische Kurzzeittherapien weniger wirksam seien (Grawe et al. 1994). Diese Ergebnisse wurden von Leichsenring (1996) und anderen Autoren aus methodischen Gründen in Frage gestellt. Aktuelle Arbeiten (Leichsenring et al. 2009, Winkelbach 2007) zeigen die Gleichwertigkeit beider Methoden. Darüber hinaus trägt das Verfahren nur zu einem gewissen, nicht sehr hohen Teil zur Prädiktion eines Behandlungsergebnisses bei. Andere ausschlaggebende Faktoren sind beispielsweise auch die Persönlichkeit und Kompetenz des Therapeuten (vgl. Wampold 2001). Bezogen auf die diagnostische Situation in Psychotherapien haben Faller et al. (2002) den Einfluss des jeweiligen Therapieverfahrens (verhaltenstherapeutisch versus psychodynamisch) auf die Einschätzung der Arbeitsbeziehung und einer möglichen Prognose durch den Therapeuten untersucht. Eine Bewertung von Seiten der Patienten wurde dabei nicht erhoben.

Zur Wirsamkeitsprüfung und Qualitätssicherung wird von mehreren Autoren die Bedeutung der Erfassung von Therapiezielen betont. „Sie sind u.a. eine wichtige Komponente zur Beurteilung des Therapieerfolgs, sie haben positiven Einfluss auf therapeutische Prozesse, schaffen gemeinsame Bezugspunkte für verschiedene therapeutische Professionen und können zur Kontinuität des Behandlungsprozesses beitragen." (Dirmaier et al. 2002, S. 25). Ähnliches stellen auch Grosse Holtforth und Grawe (2002) fest, die im Rahmen einer umfangreichen klinischen Studie eine Taxonomie zur Erfassung von Therapiezielen entwickelten (Berner Inventar für Therapieziele, BIT-T).

Therapieziele sind nicht zwangsläufig symptomorientiert. Stiles (2006) hebt in seiner Untersuchung ferner hervor, dass es in konventionellen Betrachtungsweisen von erfolgreichen Psychotherapien häufig allein um Symptomreduktion geht. Dem stellt er 
ein Konzept gegenüber, das er als „Assimilationsmodell“ (Stiles 2006, S. 389) bezeichnet. Hierbei geht es um die Integration eines Problems, was den Fortschritt einer Therapie kennzeichnet. Dabei werden sieben verschiedene Stadien definiert, welche den Therapieprozess charakterisieren.

\subsubsection{Psychotherapie bei Generalisierter Angststörung}

Leibing et al. (2003) stellten fest, dass sich aufgrund der erwähnten Vielzahl der an GAS erkrankten Patienten, des frühen Auftretens oftmals zusammen mit anderen psychischen Erkrankungen sowie der häufigen Chronifizierung ein beträchtlicher gesundheitspolitischer Stellenwert ergibt. „Das häufig eher somatische Krankheitskonzept der Patienten führt vielfach zur Inanspruchnahme verschiedenster Fachärzte, bevor eine adäquate Behandlung eingeleitet werden kann." (Wittmund et al. 2003, S. 284). Trotz dieser Bedeutung ist GAS empirisch bisher vergleichsweise wenig untersucht worden, zumal dieses Störungsbild zunehmend häufiger aufzutreten scheint (Becker und Margraf 2007).

Wie bereits erwähnt, werden viele GAS-Patienten medikamentös behandelt (z.B. durch Antidepressiva oder Benzodiazepine). Allerdings kommt es unter diesen Therapien häufig zu Rückfällen (Leichsenring et al. 2005) oder zu einer Mediakamentenabhängigkeit. Darüber hinaus existieren bisher nur wenige Studien, welche die medikamentöse Behandlung von GAS über einen längeren Zeitraum (als nur einige Wochen) untersuchen und in Katamnesen beobachten, wie sich der Verlauf der Störung nach Absetzen der Pharmakotherapie entwickelt (Becker und Margraf 2007). Eine rein medikamentöse Behandlung kann den Nachteil mit sich bringen, "negative Effekte auf die Entwicklung von Coping-Fähigkeiten“ (Winkelbach 2007, S. 27) seitens des Patienten zu haben. Es wird eine eher „passive Rolle des Patienten“ (Becker und Margraf 2007, S. 30) gefördert. Ein direkter Vergleich von VT und medikamentöser Behandlung bei GAS ergab zumeist bessere Ergebnisse für VT, besonders hinsichtlich katamnestischer Untersuchungen (Becker und Margraf 2007). Auch pharmakologisch-psychotherapeutische Kombinationstherapien der GAS sind nach bisherigen Erkenntnissen der alleinigen Psychotherapie bezüglich der Effektstärken unterlegen (vgl. Becker und Margraf 2007). Somit erhält die Psychotherapie als alternative bzw. ergänzende Option der Behandlung der GAS eine besondere Bedeutung. 
Es liegen bislang keine eindeutigen Resultate vor, welche die Bevorzugung einer bestimmten Psychotherapieform gegenüber einer anderen begründen könnten (Senf und Broda 2005). Laut Leichsenring et al. (2009) stellt sich der Forschungsstand folgendermaßen dar: Es wurden einige kontrollierte Studien durchgeführt, welche kognitive Verhaltenstherapie (CBT) bei GAS als effizient erwiesen, während zu psychodynamischer Kurzzeittherapie von GAS bislang eher wenige Untersuchungen vorliegen. Zu nennen ist hier die Studie von Crits-Christoph et al. (2004), der die Wichtigkeit der Erwartungen an den Therapeuten seitens des Patienten sowie interpersoneller Probleme als Fokus in einer psychodynamischen Behandlung betont. Positive Erwartungen der Patienten an die Psychotherapie (im Gegensatz zu jenen an eine Pharmakotherapie) wurden dabei als Prädiktoren des Therapieerfolgs identifiziert.

Leichsenring et al. (2009) stellen fest, dass bereits VT mit psychodynamischer Kurzzeittherapie bei GAS verglichen wurde, jedoch mit methodischen Einschränkungen, wie beispielsweise dem Vorhandensein eines Behandlungsmanuals nur für VT. Außerdem hatten bisherige Studien kleinere Patientenzahlen als die mit dieser Arbeit ausgewertete Studie.

Es werden mit der vorliegenden Untersuchung beide Therapieformen in Kurzzeittherapien der GAS unter gleichen Bedingungen (z.B. Manual als Anleitung beider Therapieverfahren) beurteilt.

\subsection{Zielsetzung der Studie, Fragestellung und Hypothesen}

Mit der hiermit zum Teil ausgewerteten Studie wird die verhaltenstherapeutische und psychodynamische Kurzzeittherapie von GAS-Patienten mittels verschiedener Auswertungstools untersucht. Die vorliegende Arbeit geht dabei in einer vergleichenden Untersuchung speziell der Fragestellung nach, inwiefern sich (durch die Patienten selbst auf PATH-Fragebögen formulierte) Probleme und Ziele im Verlauf der Behandlung verändern. Außerdem ist von Interesse, ob bestimmte Prädiktoren für besonders erfolgreiche (oder auch weniger erfolgreiche) Therapieverläufe identifiziert werden können und ob sich diese nach angewandtem Therapieverfahren voneinander unterscheiden.

Eine zeitgleich entstehende Dissertation (Werling 2010) vergleicht demgegenüber die PATH-Daten der GAS-Patienten dieser Studie mit denen einer StudentenStichprobe. 
Die Fragestellung wurde folgendermaßen eingegrenzt:

1. Sind im Therapieverlauf Veränderungen der durch die Patienten benannten Probleme und Ziele feststellbar? Lassen sich diese den beiden Therapieverfahren zuordnen?

2. Inwieweit stellen zu Beginn der Therapien von Patienten genannte Probleme und Ziele (siehe oben) Prädiktoren für den Erfolg der Therapie dar? Sind diese innerhalb der beiden Vergleichsgruppen (VT und SET) unterschiedlich verteilt? Nennen dabei Patienten, die in ihrer Psychotherapie erfolgreich sind, andere Probleme und Ziele?

Aus der Zielsetzung lassen sich folgende Hypothesen ableiten:

H1.1 Ziele und Probleme verändern sich im Therapieverlauf.

H1.2 Die behandelten Patienten formulieren unterschiedliche Probleme und Ziele zu Befragungszeitpunkt $\mathrm{t}$, je nachdem, ob sie verhaltenstherapeutisch oder psychodynamisch behandelt werden. Die Patienten wurden zwar randomisiert den Therapieverfahren zugewiesen, jedoch fanden vor Zeitpunkt t1 bereits drei diagnostische und therapeutische Sitzungen statt.

H1.3 Je nach Therapieverfahren (verhaltenstherapeutisch oder psychodynamisch/ SET) werden im Therapieverlauf unterschiedliche Probleme und Ziele genannt.

H1.4 Im Therapieverlauf sind systematische Veränderungen festzustellen.

Hypothesen, die auf der Gruppeneinteilung nach reduziertem Leidensdruck beruhen:

H2.1 Es gibt Prädiktoren, die für einen guten oder eher mäßigen Therapieverlauf sprechen.

H2.2 Die in Hypothese H2.1 ermittelten Prädiktoren für den Erfolg von Therapieverläufen sind innerhalb der beiden untersuchten Therapiegruppen (supportivexpressive Therapie (SET) und Verhaltenstherapie) unterschiedlich verteilt.

Bestätigen sich die Hypothesen, so würde dies bedeuten, dass sich Hinweise auf unterschiedliche Indikationsstellungen aufgrund von Untersuchungen mit PATH ergeben. Therapeutische Konsequenzen könnten sich auch für die empfohlene Dauer der Behandlung oder die Wahl eines Psychotherapieverfahrens ableiten lassen. 


\section{Material und Methoden}

\subsection{Material}

Bei dem untersuchten Material handelt es sich um im Rahmen einer von der Deutschen Forschungs-Gemeinschaft (DFG Geschäftszeichen LE 1250/1-1/1-2) geförderten Studie erhobene Daten (Leichsenring et al. 2009), welche dem Vergleich von VT und SET bei Patienten mit GAS dienen sollen.

Die Studie wurde an der Abteilung für Psychosomatische Medizin und Psychotherapie der Universität Göttingen zwischen Mai 2001 und Juni 2007 im Raum Göttingen durchgeführt. Die Datenerhebung selbst wurde nicht durch die Verfasserin dieser Arbeit, sondern durch Angehörige der Abteilung für Psychosomatische Medizin und Psychotherapie der Universität Göttingen durchgeführt. PATH-Fragebögen wurden im Rahmen der Studie wiederholt ausgeteilt. Die Auswertung dieser Daten ist Grundlage der vorgelegten Arbeit.

Die Ethikkommission der Medizinischen Fakultät der Georg-August-Universität Göttingen genehmigte die Studie. Alle Patienten wurden über die Auswertung informiert, haben ihr Einverständnis gegeben sowie dieses schriftlich dokumentiert. Die Daten unterliegen der ärztlichen Schweigepflicht und wurden vor der Auswertung anonymisiert, so dass Personen den Daten nicht zu zuordnen sind. Es besteht somit keine Gefahr oder Schädigung für die an der Studie teilnehmenden Patienten.

Die Studienteilnehmer wurden randomisiert einer der beiden Behandlungsgruppen zugewiesen.

Im Folgenden werden die verwendeten Datenerhebungsverfahren erläutert.

\subsubsection{PATH (Problems and Aims in Therapy)}

Nach Staats et al. (1996) ist PATH ein Instrument zur Erfassung von Veränderungen der Probleme und Ziele im Prozess einer psychotherapeutischen Behandlung. Grundgedanke ist, dass sich, vor allem während langer Psychotherapien, Ziele und Probleme verändern. Dies gilt insbesondere für psychodynamische Therapieverfahren, kann aber auch bei verhaltenstherapeutischen Behandlungen festgestellt werden. Der Fokus der Behandlung sollte daher nicht nur auf zu Beginn angegebene Probleme und Ziele der Patienten gerichtet sein.

Durch Modifikation ähnlicher Instrumente wurde PATH ursprünglich speziell für die Anforderungen der Auswertung von Langzeitpsychotherapien konzipiert (Frölich 
2009). Mit der vorliegenden Dissertation wird PATH nun auch bei Kurzzeittherapien und erstmals bei Patienten mit einem definierten Störungsbild (hier GAS) eingesetzt. Bei den ausgewerteten, mittels PATH erhaltenen Daten handelt es sich um frei formulierte, kurze (schriftliche) Beschreibungen ambulant behandelter Patienten zu den für sie drei wichtigsten Problemen und Zielen. Diese werden als Problem bzw. Ziel 1 bis 3 angegeben, jedoch durch die Patienten nicht danach „sortiert", welches Problem oder Ziel am dringendsten ist.

Zusätzlich wird in den Fragebögen darum gebeten, die zuvor genannten Probleme und Ziele auf einer fünfstufigen Skalierung wiederholt zu beurteilen (im Folgenden als Selbsteinschätzung bezeichnet). Dabei gilt für die Bewertung des Leidensdrucks unter angegebenen Problemen: 0 = überhaupt nicht, 1 = wenig, 2 = etwas, 3 = stark, $4=$ könnte nicht schlimmer sein. Analog bedeuten betreffend der Verwirklichung formulierter Ziele die Zahlen 0 = überhaupt nicht, $1=$ wenig, $2=$ etwas, $3=$ viel, $4=$ vollständig. Diese Angaben können beispielsweise eine Bewertung des Therapieverlaufs aus Sicht der Patienten ermöglichen.

Tabelle 1: Auszug aus PATH-Fragebogen (Frölich 2009, S. 53)

„Bitte machen Sie eine Liste der drei Probleme, unter denen Sie zur Zeit am meisten
leiden. (...) Schätzen Sie nun ein, wie sehr Sie zur Zeit unter diesen Problemen leiden.“
„Bitte geben Sie als nächstes an, welche Ziele Sie im Laufe der Therapie erreichen
möchten. Versuchen Sie bitte, diese Ziele möglichst konkret zu formulieren. Bitte geben
Sie drei dieser Ziele an.“
Im Verlauf:
"Sie haben zu Beginn der Therapie formuliert, wie Sie Ihre Probleme und Ziele damals
gesehen haben. (...) Bitte schätzen Sie nun ein, wie sehr Sie zur Zeit unter diesen Prob-
lemen leiden, bzw. inwieweit Sie die Ziele erreicht haben.“

Da mit PATH Probleme und Ziele (statt Diagnosen) berücksichtigt werden, geben die Schilderungen der Patienten näher deren tatsächliches Krankheitserleben wieder. Schließlich sind nicht alle Patienten, bei denen GAS diagnostiziert wurde, gleich. Es gibt viele verschiedene Ausprägungen und individuelle Beeinträchtigungen. Formulierte Probleme und Ziele können hier eine prädiktive Kraft haben und so für die einzelne Therapieplanung wichtige Hinweise liefern. Durch die offene Fragestellung der 
PATH-Methode werden ferner nicht nur Therapieziele angegeben, es können vielmehr auch Lebensziele der Patienten ermittelt und (falls PATH als FeedbackMethode eingesetzt würde) in der Therapie berücksichtigt werden bzw. diese evaluieren.

Eine Befragung nach PATH lässt sich gut in den Verlauf einer ambulanten Psychotherapie integrieren. Die Behandlung wird dabei durch den Patienten selbst reflektiert, indem Ziele und Probleme eigens schriftlich verfasst sowie Gedanken und Erwartungen an die Therapie konkretisiert und bilanziert werden. Darüber hinaus erhalten die Patienten durch ihre wiederholte Bewertung bereits angegebener Probleme und Ziele eine Rückmeldung über den Behandlungsprozess. Ausgefüllte PATHFragebögen werden von den Patienten in der Regel anonym zurückgesendet, die Therapeuten erhalten dabei keine Information über deren Inhalt (Staats, 2000). Die Patienten können somit unbefangen Probleme und Ziele angeben und beurteilen, inwiefern die Therapie zu deren Besserung bzw. Erreichen führte.

\subsubsection{Befragungszeitpunkte}

Die verschiedenen Therapie-Zeitpunkte der Befragung (im Folgenden auch Erhebungszeitpunkte) wurden folgendermaßen gewählt:

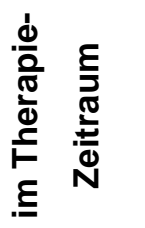

t1: 3. Therapiesitzung (Beginn der Therapie)

t2: 12. Therapiesitzung (während der Therapie)

t3: Abschlussuntersuchung (posttherapeutisch)

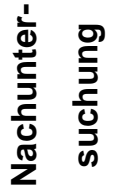

t4: 6 Monate nach Therapieende (Katamnese 1)

t5: 12 Monate nach Therapieende (Katamnese 2)

\subsubsection{Stichprobenbeschreibung}

Vor Studienbeginn wurden bestimmte Ein- und Ausschlusskriterien formuliert. Demnach war Voraussetzung für die Teilnahme an der Studie, dass die Patienten während des Studienablaufs zwischen 18 und 65 Jahre alt waren und GAS die Hauptdiagnose war. Die Hauptdiagnose wurde definiert als die hauptsächliche mentale Störung nach $A D I S-R^{3}$. Folgende Ausschlusskriterien wurden angewandt:

\footnotetext{
${ }^{3}$ ADIS-R = Anxiety Disorder Interview Schedule - Revised; strukturiertes, diagnostisches Interview
} 
1) Das Vorliegen einer akuten, instabilen oder massiven körperlichen Erkrankung (medizinische DSM-Achse-III-Störung ${ }^{4}$ ), welche möglicherweise eine erfolgreiche Therapie-Beendigung stören könnte

2) Jegliche gegenwärtige oder frühere schizophrene oder bipolare Störung, oder Gruppe-A- oder -B-Achse-II-Störung ${ }^{5}$

3) Jegliche gegenwärtige oder frühere neurologische Störung

4) Anzeichen von Alkohol- oder Substanzmissbrauch oder -abhängigkeit, einer Essstörung oder einer Major Depression innerhalb der vergangenen 12 Monate

5) Patienten, welche sich in einer gleichzeitig laufenden psychotherapeutischen oder psychopharmakologischen Behandlung befinden

Die Patienten wurden durch Empfehlungen von niedergelassenen Ärzten und Psychotherapeuten sowie durch Inserate und Informationen über die Untersuchung in den Medien für die Studie gewonnen. Alle Patienten wurden mittels Strukturierter Klinischer Interviews für DSM-IV (SCID I, II) ${ }^{6}$ diagnostiziert. Sämtliche Interviews wurden von einem erfahrenen und besonders ausgebildeten klinischen DiplomPsychologen durchgeführt. Die Diagnosestellung erfolgte bei Übereinstimmung von mindestens zwei erfahrenen klinischen Psychologen.

Insgesamt wurden 231 Patienten überprüft, von welchen 57 die Einschlusskriterien erfültten und auf die keine Ausschlusskriterien zutrafen (Leichsenring et al. 2009). Sechs dieser Patienten schieden (wegen Umzug, Problemen in der therapeutischen Beziehung, etc.) aus, sodass die Stichprobe der vorliegenden Arbeit aus $\mathrm{n}=51$ Patienten besteht, die ihre Therapie abgeschlossen haben (26 VT- und 25 SETPatienten). Nach Behandlungsende schieden drei Patienten wegen weiterer Behandlungsbedürftigkeit oder somatischer Erkrankungen aus (ein VT- und zwei SETStudienteilnehmer), sodass die Behandlung einschließlich eines einjährigen Katamnese-Zeitraumes von 25 VT- und 23 SET-Patienten absolviert wurde.

\footnotetext{
${ }^{4}$ DSM Achse III = $\quad$ Diagnostisches und Statistisches Manual Psychischer Störungen; Klassifikationssystem der American Psychiatric Association, Achse III: medizinische Krankheitsfaktoren (Häcker und Stapf 2004)

${ }^{5}$ DSM Achse II = $\quad$ Persönlichkeitsstörungen, geistige Behinderung (Häcker und Stapf 2004)

${ }^{6} \mathrm{SCID} \quad=\quad$ Structured Clinical Interview for DSM Disorders
} 
Das Durchschnittsalter der 51 Patienten lag zu Therapiebeginn bei 45 Jahren (arithmetisches Mittel), die Standardabweichung beträgt SD = 11. Die Altersspanne reichte von 21 bis 65 Jahren, 80,4\% der Studienteilnehmer sind Frauen, 19,6\% Männer, was den absoluten Häufigkeiten von 41 Frauen und 10 Männern entspricht. 84,3\% der Patienten befanden sich zum Zeitpunkt der Studie in einer festen Partnerschaft.

Patienten ohne Berufsabschluss stellten einen Gesamtanteil von 9,8\%, eine fertige Lehre hatten $64,7 \%$ und ein abgeschlossenes Hochschulstudium 25,5\% der Studienteilnehmer.

$50,98 \%$ der Patienten wurden verhaltenstherapeutisch, 49,02\% psychodynamisch (nach SET-Verfahren) behandelt. In der SET-Gruppe befanden sich zum Zeitpunkt des Therapiebeginns mehr Patienten in einer festen Partnerschaft $(p=0,02)$. Ansonsten bestehen keine signifikanten Unterschiede in der Gruppenzusammensetzung.

Tabelle 2: Zusammensetzung der Therapiegruppen

\begin{tabular}{|c|c|c|c|c|c|c|c|}
\hline & \multicolumn{3}{|c|}{$\begin{array}{c}\text { VT } \mathbf{n}=\mathbf{2 6} \\
(50,98 \% \text { der untersuchten } \\
51 \text { Patienten })\end{array}$} & \multicolumn{3}{|c|}{$\begin{array}{c}\text { SET } \mathbf{n}=25 \\
(49,02 \% \text { der untersuchten } \\
51 \text { Patienten })\end{array}$} & $\begin{array}{c}\text { Signifikanztest } \\
\text { signifikanter Unterschied in } \\
\text { Gruppenzusammen- } \\
\text { setzung, wenn } p<0,5\end{array}$ \\
\hline & $\mathbf{n}$ & \multicolumn{2}{|c|}{ \% Therapiegruppe } & $\mathbf{n}$ & \multicolumn{2}{|c|}{ \% Therapiegruppe } & p \\
\hline Frauen & 20 & \multicolumn{2}{|c|}{$76,92 \%$} & 21 & \multicolumn{2}{|c|}{$84 \%$} & \multirow[t]{2}{*}{0,52} \\
\hline Männer & 6 & \multicolumn{2}{|c|}{$23,08 \%$} & 4 & \multicolumn{2}{|c|}{$16 \%$} & \\
\hline in fester Partnerschaft & 19 & \multicolumn{2}{|c|}{$73,08 \%$} & 24 & \multicolumn{2}{|c|}{$96 \%$} & 0,02 \\
\hline & \multicolumn{2}{|c|}{$\begin{array}{c}\text { Durchschnitt } \\
\text { in Jahren }\end{array}$} & SD & \multicolumn{2}{|c|}{$\begin{array}{c}\text { Durchschnitt } \\
\text { in Jahren }\end{array}$} & SD & \\
\hline $\begin{array}{l}\text { Alter bei } \\
\text { Therapiebeginn }\end{array}$ & \multicolumn{2}{|r|}{44,65} & 11,57 & \multicolumn{2}{|r|}{44,46} & 11,27 & 0,87 \\
\hline
\end{tabular}

Für 16 der 51 Studienteilnehmer stellte GAS die einzige Diagnose einer mentalen Störung dar (entspricht 31,37\%), folglich bestand bei 35 der Patienten eine Kombination von GAS und mindestens einer zusätzlichen komorbiden Störung (entspricht 68,63\%). Hierbei waren andere Angsterkrankungen (33,33\%) und depressive Störungen $(23,53 \%)$ die häufigsten komorbiden Störungsbilder. Es zeigte sich außerdem, dass einige der Patienten unter komorbiden Anpassungsstörungen (15,69\%), Zwangsstörungen $(11,76 \%)$ oder somatoformen Störungen $(7,84 \%)$ litten. 


\subsubsection{Teilnehmende Psychotherapeuten}

An der Studie nahmen neun niedergelassene Psychotherapeuten teil, wovon drei weiblich waren. Die Therapeuten arbeiteten bereits vor Studienbeginn entweder mit VT oder psychodynamischer Psychotherapie als Behandlungsverfahren. Vor Studienbeginn wurden sie mit den Manualen der jeweiligen Therapierichtung für GAS speziell geschult. Die Umsetzung der Manuale während der ambulanten Therapien im Rahmen der Studie wurde sichergestellt, indem regelmäßige GruppenSupervisionen stattfanden.

Das durchschnittliche Alter der Therapeuten lag zu Therapiebeginn bei 47,9 Jahren mit einer Altersspanne von 41 bis 53 Jahren). Die durchschnittliche Berufserfahrung lag bei den verhaltenstherapeutisch arbeitenden Psychotherapeuten bei 18,7 Jahren (Spanne von zwölf bis 30 Jahren) und bei den psychodynamisch vorgehenden SETTherapeuten bei 16,3 Jahren (Spanne von vier bis 26 Jahren).

\subsection{Auswertungsmethoden}

Die Beurteilung der Angaben aus den PATH-Fragebögen erfolgte mit Hilfe zweier Skalen, dem Kategoriensystem und der Mastery-Awareness-Scale. Mittels dieser Instrumente wurden die von Patienten formulierten Probleme und Ziele durch auswertende Personen (Doktorandinnen Verena Werling und Frauke Wiegand, im Folgenden Rater) sinnvoll den verschiedenen Kategorien der Skalen zugeteilt. Zusätzlich fand eine Auswertung der Selbsteinschätzungs-Aussagen der Studienteilnehmer statt. Die Entwicklung des (wiederholt durch die Patienten selbst beurteilten) Leidensdrucks unter den genannten Problemen bzw. der Zielverwirklichung der zuvor genannten Ziele kann so über den gesamten Behandlungszeitraum hinweg beurteilt werden. Dies ermöglicht eine quantitative Begutachtung des Therapieverlaufs.

Die Rater verfügten über keine besonderen Vorkenntnisse im Bereich der Psychotherapieforschung. Durch ein Training der Rater wurde die korrekte und zuverlässige Anwendung der Auswertungsskalen auf PATH-Daten gewährleistet.

Wie bereits angegeben, waren die Antwortbögen selbst anonymisiert, so dass die Rater keine Rückschlüsse auf persönliche Daten der Patienten ziehen konnten. Im Folgenden werden die beiden verwendeten Skalen näher erläutert. 


\subsubsection{Kategoriensystem}

Das Kategoriensystem zur qualitativen Inhaltsanalyse frei verfasster PatientenAussagen wurde von Frölich (2009) publiziert. Mit diesem Instrument können eventuell stattfindende Veränderungen von Problemen und Zielen im Therapieverlauf untersucht werden. Es gliedert sich in jeweils zehn Problem- und Zielkategorien (siehe folgende Tabelle). Meist deckt eine Kategorie (je 1 bis10) inhaltlich Probleme und Ziele aus ähnlichen Bereichen ab. Die Problem- und Zielangaben eines Patienten müssen in den Kategorien jedoch nicht zwangsläufig einander entsprechen.

Zur klaren Einordung der Patienten-Aussagen in die unterschiedlichen Kategorien wurden Ankerbeispiele und Kodierregeln verfasst (Frölich 2009).

Tabelle 3: Kategoriensystem (Frölich 2009, S. 22-24)

\section{Probleme}

P1 körperliche Beschwerden: Äußerungen/ Beschwerden/ Probleme/ Symptome betreffend, auch Diagnosen, Sexual- und Schlafstörungen, Albträume

P2 depressive Beschwerden: Überlastung, Erschöpfung, keine Entspannung, fehlender Antrieb, Einsamkeit, Gefühl der Gefühllosigkeit, sich selber nichts gönnen, Darniederliegen von Stimmungen, innere Getriebenheit, die zu Lähmung führt; Suizidalität

P3 Angst: Alle Textstellen, in denen Angst deutlich genannt wird, kein Vermeidungsverhalten

\section{P4 Sucht, Essstörung}

P5 mangelndes Selbstwertgefühl, Selbstvertrauen: Nur, wenn Begriff eindeutig genannt

P6 ungelöste Probleme, Konflikte: (z.B. Alltag und Beruf)

Äußerer Anteil der Konflikte oder Probleme wird beschrieben oder eine konkrete Situation genannt, alle genannten beruflichen Probleme, auch, wenn sie z.B. mit depressiven Beschwerden einhergehen P7 sonstige selbstbezogene Probleme: Eigene Befindlichkeit, unzufrieden, unglücklich, Gefühle nicht ausdrücken können

P8 interpersonelle Probleme: Andere werden explizit genannt

Differenzierung in Sub-Kategorien:

P8K Kontrolle/ Unterwerfung (moving against others): Andere kontrollieren wollen, Autorität Anderer schwer anzuerkennen, Machtkämpfe, aber auch sich unterwerfen

P8D Distanz/ Nähe (moving from others): Schwierigkeiten Kontakt zu knüpfen, sich Anderen zu öffnen, aber auch sich nicht lösen können

P8S Selbstaufgabe/ -bild (moving towards others): Angst vor Ablehnung, Wunsch nach Anerkennung; Erwartungen Anderer erfüllen wollen, sich ausnutzen lassen

P8X sonstige bzw. nicht näher zuzuordnende interpersonelle Probleme

P9 existentielle Probleme: Nach Yalom Grundfragen von Leben und Tod, das Leben ist ungerecht, man ist allein, man trägt die Verantwortung für sein Handeln, man kann gewissen Nöten nicht entgehen, älter werden 
P10 Zwänge: Entscheidungsschwierigkeiten, ausgeprägte und lähmende Ambivalenzen, auch, wenn dies als Angst oder Befürchtung beschrieben wird, jedoch ohne depressive Komponente (dann P2); Perfektionsansprüche, übertriebenes Verantwortungsgefühl

\section{$\underline{\text { Ziele }}$}

Z1 körperliche Beschwerden bessern

Z2 depressive Beschwerden bessern

Z3 Angst bewältigen, Mut entwickeln

Z4 Sucht, Essstörung bewältigen

Z5 Selbstvertrauen stärken (siehe P5)

Z6 (äußere) Probleme bewältigen: Beruf, Finanzen, Alltag

Z7 Ruhe/ Gelassenheit finden: Mit sich in Einklang kommen, sich selbst erkennen, Lebensfreude/

Glück/ Zufriedenheit erlangen, Gefühle ausdrücken/ regulieren/ zulassen

Z8 interpersonelle Probleme lösen (entsprechend P8)

Z8K Andere weniger kontrollieren wollen, Autoritäten akzeptieren lernen, aber auch lernen, sich durchzusetzen

Z8D Distanz überwinden; sich Anderen öffnen, Hilfe annehmen, aber auch allein sein können

Z8S Sich abgrenzen lernen, selbstbestimmt handeln, eigene Maßstäbe setzen, Kritik äußern

ZX Sonstige interpersonelle Probleme lösen

Z9 Freiheiten, Fähigkeiten erlangen, sonstige Ziele erreichen

Z10 Zwänge abbauen, Ambivalenzen überwinden; sich entscheiden lernen (nur, wenn depressive Komponente fehlt

In der vorliegenden Dissertation werden die entsprechenden Problemkategorien mit einem „P“ versehen, Zielkategorien mit einem „Z“ (z.B. P1 = Problemkategorie „körperliche Beschwerden").

\subsubsection{Mastery-Awareness-Scale}

Das Auswertungsinstrument der Mastery-Awareness-Scale weist Ähnlichkeiten zur Heidelberger Umstrukturierungsskala (Rudolf et al. 2000) auf, allerdings werden die Ebenen der Wahrnehmung und Bewältigung getrennt voneinander beurteilt. Dahlbender et al. (2001) entwarfen eine Methode zur Beurteilung des Zentralen Beziehungskonflikts, die sogenannte Mastery Scale. „Meisterung wird hier verstanden als die Fähigkeit zur emotionalen Selbstkontrolle und zum kognitiven Selbstverständnis im Rahmen zwischenmenschlicher Beziehungen." (Dahlbender et al. 2001, S. 176).

Mit der Mastery-Awareness-Scale (Problemwahrnehmungs- und Problembewältigungs-Skala) kann darüberhinausgehend überprüft werden, ob sich die Wahrneh- 
mung und Einflussnahme auf die genannten Probleme mit der Zeit verändern. Es werden mit dieser Skalierung somit nur Problemaussagen untersucht.

Die Mastery-Awareness-Scale wurde im Rahmen der Entwicklung und Erprobung von PATH konzipiert. Die Weiterentwicklung durch Staats führte zu der in dieser Dissertation angewandten Version mit je vier Wahrnehmungs- und Bewältigungskategorien (Staats 2009), welche auf einer vorläufigen, bereits publizierten Fassung (Frölich 2009) basiert. Die Kategorien gliedern sich bei der Problemwahrnehmung in „nicht greifbares Problem“, „vage Problemwahrnehmung“, „greifbares Problem“ und „abgrenzbares Problem: Zusammenhänge werden gesehen bzw. äußere Probleme“. Die Problembewältigung wird unterteilt in „Hilflosigkeit, Ausweglosigkeit“, „noch keine Bewältigungsmöglichkeit“, „Handlungs- und Bewältigungsmöglichkeit“ sowie „abgrenzbares Problem: aktive Einflussnahme bzw. selbstverständlicher Umgang mit Problemen“.

Tabelle 4: Mastery-Awareness-Scale (Staats 2009)

\begin{tabular}{|c|c|c|c|c|c|}
\hline \multirow{2}{*}{$\begin{array}{l}\text { Wahrnehmung } \\
\text { (Awareness) }\end{array}$} & \multirow{2}{*}{$\begin{array}{l}1 . \\
\text { Nicht greifba- } \\
\text { res Problem }\end{array}$} & \multirow{2}{*}{$\begin{array}{l}2 . \\
\text { Vage Problem- } \\
\text { wahrnehmung }\end{array}$} & \multirow{2}{*}{$\begin{array}{l}3 . \\
\text { Greifbares } \\
\text { Problem }\end{array}$} & \multicolumn{2}{|c|}{ 4. Abgrenzbares Problem } \\
\hline & & & & $\begin{array}{l}\text { a) } \\
\text { Zusammen- } \\
\text { hänge wer- } \\
\text { den gesehen }\end{array}$ & $\begin{array}{l}\text { b) } \\
\text { Äußere } \\
\text { Probleme }\end{array}$ \\
\hline $\begin{array}{l}\text { Erläuterung + } \\
\text { Ankerbeispiele }\end{array}$ & $\begin{array}{l}\text { Beschwerden } \\
\text { werden be- } \\
\text { schrieben } \\
\text { Zusammen- } \\
\text { hänge zwischen } \\
\text { Problem und } \\
\text { Beschwerden } \\
\text { werden nicht } \\
\text { gesehen } \\
\text { Bsp. „tiefe Trau- } \\
\text { rigkeit“, Schuld- } \\
\text { gefühle, körper- } \\
\text { liche Be- } \\
\text { schwerden }\end{array}$ & $\begin{array}{l}\text { sieht Zusam- } \\
\text { menhang zwi- } \\
\text { schen Problem } \\
\text { und Beschwer- } \\
\text { den } \\
\text { Problem wird } \\
\text { umschrieben }\end{array}$ & $\begin{array}{l}\text { zeigt Problem- } \\
\text { bewusstsein } \\
\text { sieht Problem } \\
\text { als zu sich ge- } \\
\text { hörig } \\
\text { Bsp.: „muss } \\
\text { meine Frau vor } \\
\text { den Kopf stos- } \\
\text { sen, so bin ich } \\
\text { halt“ }\end{array}$ & $\begin{array}{l}\text { „muss Schall- } \\
\text { plattensamm- } \\
\text { lung sortieren, } \\
\text { um mich ab- } \\
\text { zureagieren“ }\end{array}$ & $\begin{array}{l}\text { akzeptierende } \\
\text { Wahrnehmung }\end{array}$ \\
\hline
\end{tabular}




\begin{tabular}{|c|c|c|c|c|c|}
\hline \multirow{2}{*}{$\begin{array}{l}\text { Mastery } \\
\text { (Bewältigung) }\end{array}$} & \multirow{2}{*}{$\begin{array}{l}1 . \\
\text { Hilflosigkeit, } \\
\text { Ausweglosig- } \\
\text { keit }\end{array}$} & \multirow{2}{*}{$\begin{array}{l}. \\
\text { Noch keine } \\
\text { Bewältigungs- } \\
\text { möglichkeit }\end{array}$} & \multirow[b]{2}{*}{\begin{tabular}{|l} 
Handlungs- \\
und \\
Bewältigungs- \\
möglichkeit
\end{tabular}} & \multicolumn{2}{|c|}{ 4. Abgrenzbares Problem } \\
\hline & & & & $\begin{array}{l}\text { a) } \\
\text { Aktive Ein- } \\
\text { flussnahme }\end{array}$ & $\begin{array}{l}\text { b) } \\
\text { Selbstver- } \\
\text { ständlicher } \\
\text { Umgang mit } \\
\text { Problemen }\end{array}$ \\
\hline $\begin{array}{l}\text { Erläuterung + } \\
\text { Ankerbeispiele }\end{array}$ & $\begin{array}{l}\text { „Sog, aus dem } \\
\text { man nicht ent- } \\
\text { kommt“, „,ühle } \\
\text { mich verloren“, } \\
\text { „Selbstmordge- } \\
\text { danken“ }\end{array}$ & $\begin{array}{l}\text { Patient sieht } \\
\text { sich nicht als } \\
\text { aktiv handelnd } \\
\text { und auch nicht } \\
\text { dazu in der } \\
\text { Lage } \\
\text { „weiterhin häu- } \\
\text { fig“, „müsste } \\
\text { aktiver sein“, } \\
\text { "hat sich wenig } \\
\text { geändert“ }\end{array}$ & $\begin{array}{l}\text { muss sich diese } \\
\text { immer wieder } \\
\text { bewusst ma- } \\
\text { chen } \\
\text { „kann nur } \\
\text { schwer abrü- } \\
\text { cken“ }\end{array}$ & $\begin{array}{l}\text { ist sich seiner } \\
\text { Einflussmög- } \\
\text { lichkeiten } \\
\text { bewusst }\end{array}$ & $\begin{array}{l}\text { sieht Problem } \\
\text { als lustvolle } \\
\text { Herausforde- } \\
\text { rung }\end{array}$ \\
\hline
\end{tabular}

Die entsprechenden Problemkategorien werden mit einem "W“ für Wahrnehmung oder einem "M“ für Mastery (Bewältigung) versehen (z.B. W2 = Problemwahrnehmungskategorie „vage Problemwahrnehmung“).

\subsubsection{Kodierung und Auswertung}

Zwei Rater kodierten unabhängig voneinander die Angaben der Patienten nach dem Kategoriensystem und der Mastery-Awareness-Scale. Das bedeutet, dass die schriftlichen Aussagen der Patienten Kategorien der jeweiligen Skala zugeordnet wurden. Dabei waren Doppelkodierungen einer Problem- oder Zielangabe innerhalb einer Skala (Kategoriensystem, Mastery-Awareness-Scale mit Problemwahrnehmung und Problembewältigung) unzulässig.

Die Interrater-Übereinstimmung der Ergebnisse wurde kontrolliert, indem das Cohens Kappa (Bortz 2005) berechnet wurde. Dabei gilt folgende Einstufung der einzelnen Werte:
$0,0-0,39$
ungenügend
$0,4-0,59$
befriedigend
$0,6-1,0$
sehr gut 
Es ergibt sich für die Kodierung nach dem Kategoriensystem der Probleme Kappa = 0,79 bei einer prozentualen Übereinstimmung der Rater von 82,79\%. Für die Auswertung aller genannter Ziele beträgt das Kappa $=0,75$ und die prozentuale Übereinstimmung 79,42\%. Bezüglich der Kappa Werte der Auswertung mittels MasteryAwareness-Scale ergeben sich für die Wahrnehmung Kappa = 0,43 mit prozentualer Übereinstimmung von 63,07\% und für die Bewältigung Kappa $=0,47$ bei einer prozentualen Übereinstimmung von $66,83 \%$.

Bei Aussagen der Studienteilnehmer, zu denen keine Übereinstimmung im Rating erzielt wurde, fand ein sogenanntes Konsensus-Rating statt, indem die betreffenden Texte gemeinsam mit beiden Ratern diskutiert und im Konsens eine Kodierung ermittelt wurde.

Nach der Kodierung der Texte erfolgte die Auswertung dieser Daten mit der Software SPSS 17 (IBM), Excel 2007 (Microsoft) und STATISTICA Version 8 (StatSoft). Dazu wurde die Anzahl der Probleme und Ziele einer jeden Kategorie zu den Zeitpunkten $\mathrm{t} 1$ bis $\mathrm{t} 5$ ermittelt. Die Häufigkeit dieser Problem- oder Zielnennungen jeder Kategorie wurde außerdem zur Gesamtzahl der zum jeweiligen Zeitpunkt kodierten Problemoder Zielaussagen in Bezug gesetzt, sodass zusätzlich ein Prozentsatz die Verteilung der einzelnen Kategorien wiedergibt.

Darüber hinaus wurden der Leidensdruck und das Erreichen angegebener Ziele als Selbsteinschätzung der Patienten im Verlauf analysiert. Zur Gegenüberstellung des Leidensdrucks unter zu t1 angegebenen Problemen bzw. der Zielverwirklichung zu t1 genannter Ziele verschiedener Zeitpunkte bei der Überprüfung von Hypothese H1.4 wurde der Sign-Test (Vorzeichen-Test) angewandt. Dies ist ein statistisches Testverfahren für nichtparametrische Daten und anwendbar für Signifikanztests zweier abhängiger Verteilungen (Häcker und Stapf 2004).

Der Vergleich der Selbsteinschätzungen des Leidensdrucks unter zu t1 genannten Problemen und des Erreichens zu t1 angegebener Ziele beider Behandlungsgruppen zu einzelnen Zeitpunkten wurde mit dem Mann-Whitney-U-Test durchgeführt. Hierbei handelt es sich ebenfalls um ein Testverfahren für nichtparametrische Daten. Es können so Signifikanztests zweier unabhängiger Verteilungen durchgeführt werden (Häcker und Stapf 2004). Unabhängige Verteilung bedeutet in diesem Fall, dass die Patienten nur in eine der beiden Therapiegruppen randomisiert wurden, da sie entweder verhaltenstherapeutisch oder psychodynamisch behandelt wurden. 
Die p-Werte wurden schließlich gemäß Bonferroni adjustiert, um eine zu hohe Anzahl falsch positiver Testentscheidungen zu vermeiden. Mit dieser Rechnung wird das Signifikanzniveau (siehe unten) an die Anzahl durchgeführter Tests angepasst. Dabei wird der Wert des Testniveaus durch die Anzahl durchgeführter Testungen dividiert (Höfler 2004). Multiples Testen fand in der Prüfung von Hypothese H1.4 beispielsweise im Vergleich des Leidensdrucks oder der Zielverwirklichung verschiedener Zeitpunkte miteinander statt. Das globale Signifikanzniveau wurde mit alpha = $5 \%$ festgelegt, Testergebnisse mit einem Bonferroni-korrigierten Signifikanzniveau von alpha $=0,05 / 3=0,017$ können als signifikant bezeichnet werden.

Die Definitionen weiterer statistischer Begriffe können im Anhang dieser Arbeit unter 7.3.2 nachgelesen werden.

\subsubsection{Literaturrecherche}

Zunächst wurden im Zuge der Literatursuche Arbeiten im Zusammenhang mit PATH und den verwendeten Rating-Skalen sowie vergleichbaren Auswertungsmethoden evaluiert. Im weiteren Verlauf wurden wichtige Fragestellungen wie z.B. Probleme und Ziele im Rahmen einer Psychotherapie über Lehrbücher, Artikel in Fachzeitschriften und Recherche im Internet erörtert. Dabei wurden vorrangig InternetDatenbanken wie PubMed ${ }^{7}$, PSYNDEX ${ }^{8}$, oder MEDLINE ${ }^{9}$ verwendet.

\footnotetext{
${ }^{7}$ www.pubmed.gov (17.03.2010)

${ }^{8}$ www.zpid.de/index.php?wahl=PSYNDEX (17.03.2010)

${ }^{9}$ www.medline.de (17.03.2010)
} 


\section{Darstellung der eigenen Untersuchungen, Ergebnisse}

Die aufgestellten Hypothesen werden im Folgenden überprüft. Dabei sind die Ergebnisse meist deskriptiv dargestellt, die Signifikanz der Veränderungen und Unterschiede von Problemen und Zielen wurde im Rahmen dieser Arbeit nur partiell überprüft.

\subsection{Hypothesen}

H1.1 Ziele und Probleme verändern sich im Therapieverlauf.

H1.2 Die behandelten Patienten formulieren unterschiedliche Probleme und Ziele zu Befragungszeitpunkt $\mathrm{t}$, je nachdem, ob sie verhaltenstherapeutisch oder psychodynamisch behandelt werden. Die Patienten wurden zwar randomisiert den Therapieverfahren zugewiesen, jedoch fanden vor Zeitpunkt $\mathrm{t} 1$ bereits drei diagnostische und therapeutische Sitzungen statt.

H1.3 Je nach Therapieverfahren (verhaltenstherapeutisch oder psychodynamisch/ SET) werden im Therapieverlauf unterschiedliche Probleme und Ziele genannt.

H1.4 Im Therapieverlauf sind systematische Veränderungen festzustellen.

H2.1 Es gibt Prädiktoren, die für einen guten oder eher mäßigen Therapieverlauf sprechen.

H2.2 Die in Hypothese H2.1 ermittelten Prädiktoren für den Erfolg von Therapieverläufen sind innerhalb der beiden untersuchten Therapiegruppen (supportivexpressive Therapie (SET) und Verhaltenstherapie) unterschiedlich verteilt.

\subsection{Erläuterung zur Darstellung der Ergebnisse}

Um die Darstellung der Ergebnisse verständlich zu machen, wird an dieser Stelle eine Abbildung beispielhaft näher erklärt. 
Abbildung 1: Beispiel Abbildung

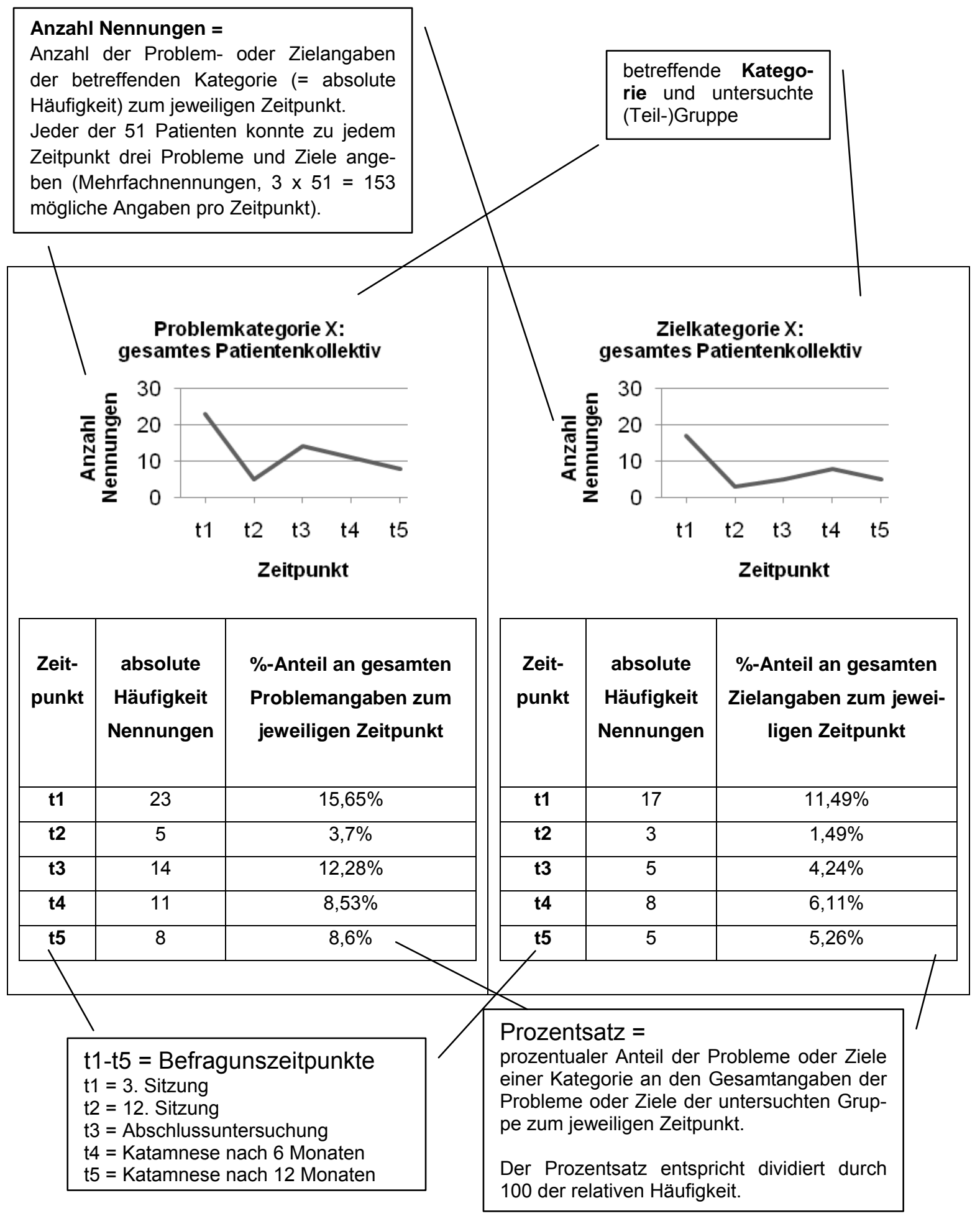

Absolute Häufigkeit bzw. Anzahl Nennungen bedeutet die absolute Häufigkeit der genannten Probleme oder Ziele einer betreffenden Kategorie zum jeweiligen Zeitpunkt. Dabei ist zu beachten, dass jeder Patient zu jedem Erhebungszeitpunkt nach drei Problemen und Zielen gefragt wurde, weshalb mitunter die Anzahl der Studien- 
teilnehmer $(n=51)$ durch die Problem- oder Zielangaben einer Kategorie überschritten wird (Mehrfachnennungen einer Kategorie aufgrund der Zuordnungen von bis zu drei Problemen oder Zielen pro Patient und Befragungszeitpunkt möglich).

\%-Anteil ist der Prozentsatz von Problemen oder Zielen der betreffenden Kategorie an allen zum jeweiligen Zeitpunkt gemachten Problem- oder Zielaussagen der Patienten einer untersuchten (Teil-)Gruppe oder des gesamten Patientenkollektives (einschließlich der Angabe, momentan "keine Probleme" oder "keine Ziele" zu haben). Der Prozentsatz drückt also den Anteil der Nennungen einer Problem- oder Zielkategorie an den tatsächlich gemachten Problem- oder Zielnennungen zu einem Zeitpunkt aus. Diese Form der Darstellung wurde gewählt, da an den unterschiedlichen Erhebungszeitpunkten nicht immer die gleiche Anzahl an Antworten in den Fragebögen vorlag und somit fehlende Angaben zu den entsprechenden Messzeitpunkten berücksichtigt werden. Im Verlauf der Studie nahm die Anzahl fehlender Angaben zu (siehe dazu Erläuterung unter 3.9).

Wird eine Teilgruppe der Studienteilnehmer (z.B. VT-Patienten, SET-Patienten, Frauen, Männer) näher analysiert, so bezieht sich der Prozentsatz auf die Problemoder Zielangaben dieser Gruppe (als 100\%) zum jeweiligen Zeitpunkt. Dabei wurden folgende Daten zu Grunde gelegt:

- Gesamtes Patientenkollektiv 51 Patienten $=51 \times 3$ = 153 mögliche Problem- oder Zielangeben zu jedem Zeitpunkt

- Verhaltenstherapeutisch wurden 26 Patienten behandelt $=26 \times 3=78$ mögliche Problem- oder Zielangeben zu jedem Zeitpunkt

- Psychodynamisch wurden 25 Patienten behandelt $=25 \times 3=75$ mögliche Problem- oder Zielangeben zu jedem Zeitpunkt

- 41 der teilnehmenden Patienten sind Frauen = 41 × 3 = 123 mögliche Problem- oder Zielangeben zu jedem Zeitpunkt

- 10 der teilnehmenden Patienten sind Männer $=10 \times 3=30$ mögliche Problem- oder Zielangeben zu jedem Zeitpunkt

$\mathrm{Da}$ (wie bereits erwähnt) von den Studienteilnehmern nicht immer alle drei gefragten Probleme und Ziele angegeben wurden, müssen für die Berechnung des Prozentsat- 
zes die fehlenden Werte von den möglichen 153 Angaben (pro Zeitpunkt, jeweils für Probleme und Ziele) subtrahiert werden.

Die Prozentsätze der Kategorien des Kategoriensystems und der MasteryAwareness-Scale werden zudem als Kreisdiagramme dargestellt, bei denen $100 \%$ allen zum jeweiligen Zeitpunkt gemachten Problem- oder Zielaussagen der untersuchten Gruppe entsprechen. Wegen der auf zwei Nachkommastellen gerundeten Ergebniswerte ergibt die Summe der Kategorien eines Kreisdiagramms in Einzelfällen bis auf wenige Nachkommastellen mehr oder weniger als 100\% (beispielsweise $100,01 \%$ oder $99,99 \%)$.

Rechnungsbeispiel: Angaben einer (Problem- oder Ziel-)Kategorie als prozentualer Anteil an allen zu einem Zeitpunkt gemachten Angaben

Untersucht wird Kategorie X und ihre Verteilung in der SET-Therapiegruppe zum Erhebungszeitpunkt $\mathrm{t} 1$.

Probleme der Kategorie $X$ wurden zum gefragten Zeitpunkt nur von einem nach SET therapierten Patienten angegeben. Dieser nannte zwei Probleme der Kategorie X. Zu t1 machte außerdem einer der 25 SET-Patienten nur zwei statt drei möglichen Problemangaben.

- absolute Häufigkeit (= Anzahl Nennungen) Kategorie X zu t1, SET-Gruppe: 2 - \%-Anteil an allen Problemangaben der SET-Patienten zum Zeitpunkt t1: 2,7\%

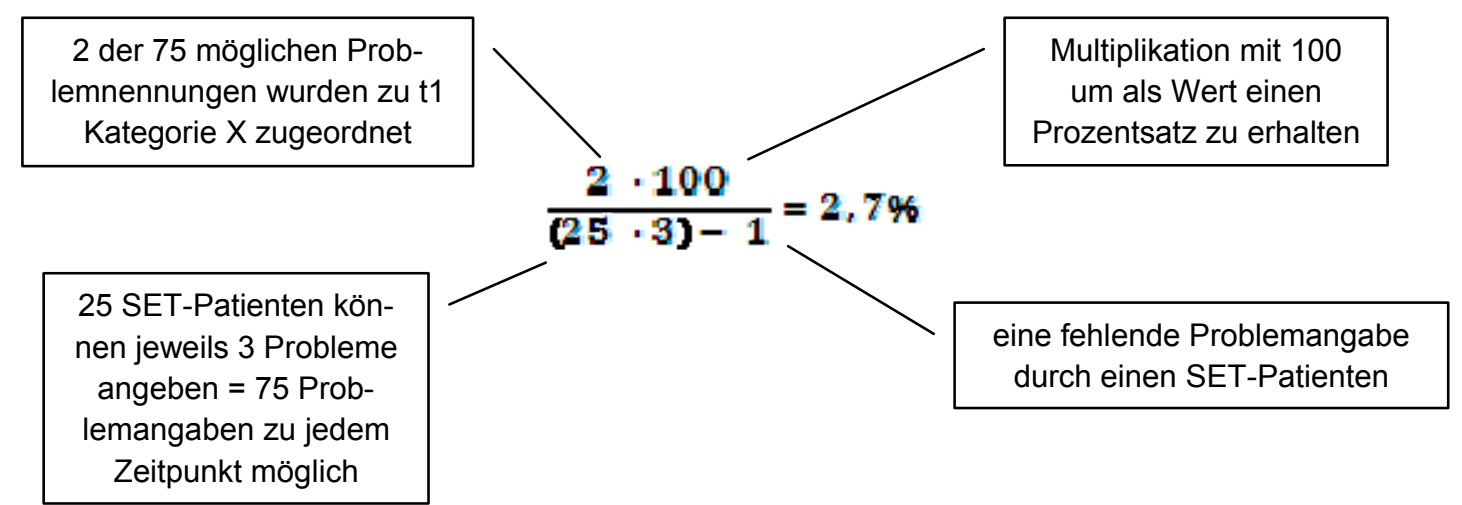

Da Prozentsatz und absolute Häufigkeit (= Anzahl Probleme oder Ziele einer Kategorie) angegeben werden, kommt gelegentlich vor, dass sich im zeitlichen Verlauf der Studie in der absoluten Häufigkeit z.B. im Diagramm kurzzeitig keine Änderung ergibt, wohl aber der Prozentsatz z.B. steigt. Die ist darin begründet, dass der Anteil 
der fehlenden Angaben mit der Zeit zunimmt (siehe Abschnitt 3.9 „Motivation zur Studienteilnahme“).

Außerdem werden oftmals höhere Werte einer Kategorie verzeichnet, als Patienten in der untersuchten Gruppe sind. Dies hat (wie bereits zuvor erläutert) seinen Ursprung in der Tatsache, dass ein Patient zu jedem Befragungszeitpunkt drei Probleme und Ziele angeben kann, die auch Inhalte derselben Kategorie enthalten können (Mehrfachnennungen, pro Patient so bis zu drei Angaben derselben Problem- oder Zielkategorie möglich). In der Grafik einer Kategorie werden zum jeweiligen Zeitpunkt alle Probleme oder Ziele der untersuchten Gruppe zu einem Gesamtgraphen addiert.

\subsection{Prüfung von Hypothese H1.1}

„Ziele und Probleme verändern sich im Therapieverlauf.“

Diese Hypothese überschneidet sich thematisch mit der zeitgleich entstehenden Dissertation von Verena Werling (Werling 2010), welche sich ebenfalls mit der Auswertung der dieser Arbeit zugrunde liegenden Studiendaten beschäftigt. Die in der folgenden Hypothese dargestellte Entwicklung der Patienten-Aussagen ist für die Einordnung weiterer Einzelergebnisse essentiell. Gleichwohl wurden die Hypothesen beider Dissertationen separat geprüft.

Die Hypothese wurde in erster Linie durch das Kategoriensystem untersucht, da dieses Instrument eine inhaltliche Beurteilung der Patientenangaben mittels zehn Kategorien ermöglicht.

Die Mastery-Awareness-Scale und die Selbsteinschätzungs-Ergebnisse der Studienteilnehmer wurden schwerpunktmäßig für die Prüfung anderer Hypothesen genutzt, da sie eher den Umgang mit Problemen und Zielen dokumentieren, jedoch nicht, ob sich diese inhaltlich verändern.

\subsubsection{Verteilung von allen genannten Problemen und Zielen auf Inhaltskatego- rien des Kategoriensystems}

Um einen ersten Überblick der Inhalte von Problemen und Zielen zu verschiedenen Zeitpunkten zu vermitteln, wurde die Darstellung mittels mehrerer Kreisdiagramme gewählt. Dabei werden zum jeweiligen Erhebungszeitpunkt $\mathrm{t} 1$ bis $\mathrm{t} 5$ alle gemachten 
Problem- oder Zielaussagen als 100\% gewertet, der Prozentsatz einer Kategorie bezieht sich also auf den Anteil an den getätigten Problem- oder Zielaussagen eines jeden Zeitpunktes. Bei Kategorie „keine Probleme“ oder „keine Ziele“ handelt es sich um Angaben von Patienten, welche ausdrücken, momentan kein Problem oder Ziel zu haben. Davon abzugrenzen sind fehlende Aussagen (Weiteres unter 3.9).

Abbildung 2: Verteilung zu t1 und t5 genannter Probleme und Ziele auf die Inhaltskategorien des Kategoriensystems (alle Patienten). Alle zum jeweiligen Zeitpunkt gemachten Problem- oder Zielaussagen entsprechen im Kreisdiagramm 100\%.

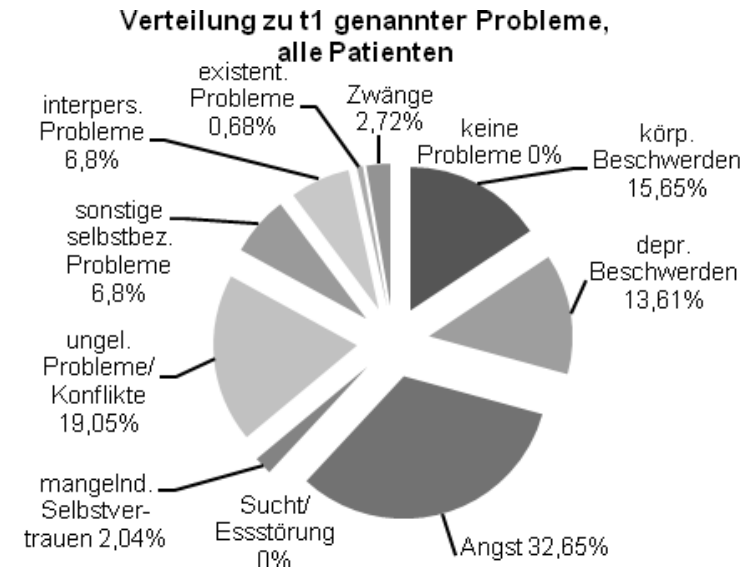

Verteilung zu t1 genannter Ziele, alle Patienten

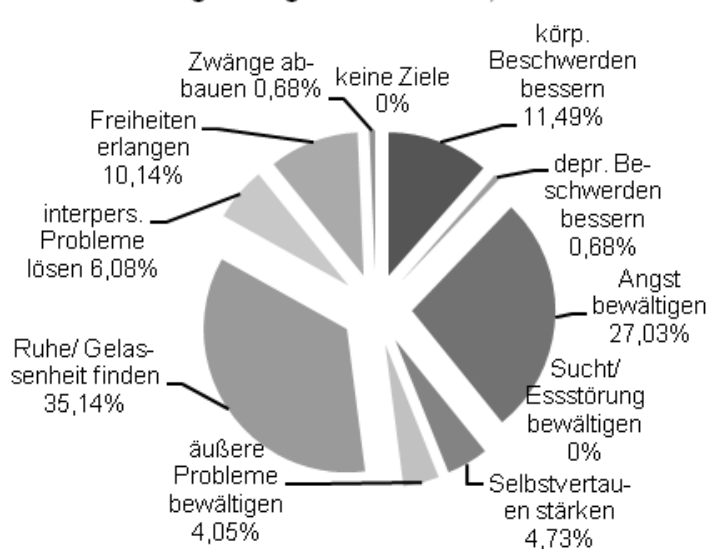

Verteilung zu t5 genannter Probleme, alle Patienten

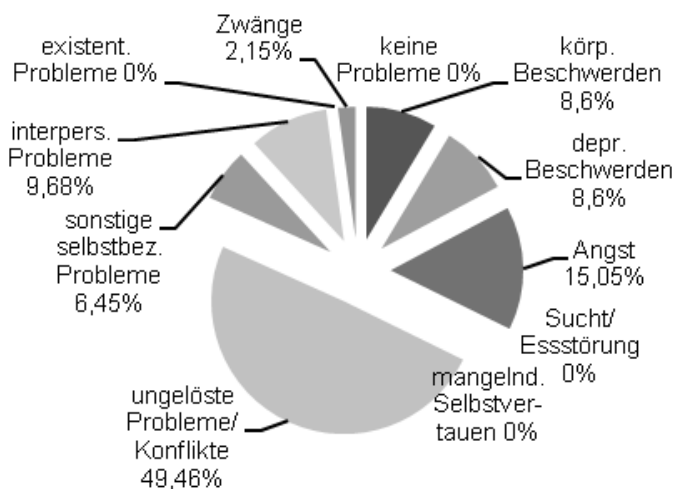

Verteilung zu t5 genannter Ziele, alle Patienten

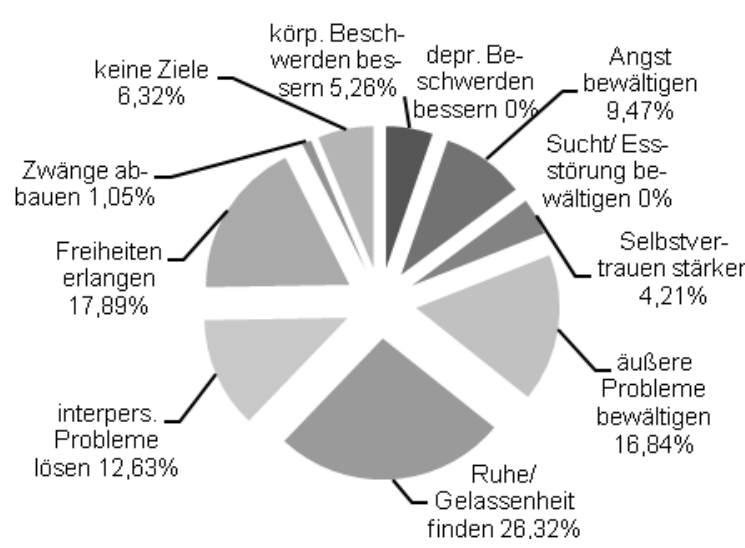

Weitere Kreisdiagramme, wie die Verteilung zu Befragungszeitpunkt t2, t3 und t4 befinden sich im Anhang dieser Arbeit unter 7.1.1.

Im Folgenden werden einzelne Kategorien genauer analysiert und ihre absoluten Häufigkeiten im gesamten Therapieverlauf dargestellt.

\section{Körperliche Beschwerden:}


Ein Beispiel für deutliche Veränderungen im Therapieverlauf ist die Kategorie „körperliche Beschwerden" (P1) des Kategoriensystems. Zu Behandlungsbeginn (Zeitpunkt t1) liegt der Anteil dieser Kategorie an allen genannten Problemen bei 15,65\% und an allen angegebenen Zielen bei 11,49\%. Nach der 12. Sitzung (t2) sinken die Probleme mit körperlichen Beschwerden auf 3,7\%, die Ziele auf 1,49\% aller Angaben. $\mathrm{Zu}$ Therapieende (t3) kann ein Anstieg bei den Problemen auf 12,28\% verzeichnet werden (Ziele, körperliche Symptome zu bessern liegen hier bei 4,24\%), im Folgejahr nach der Therapie sinkt dies Kategorie jedoch wieder, der Problemanteil liegt zu t5 bei 8,6\% und die Ziele bei 5,26\%.

Abbildung 3: zeitliche Entwicklung von Kategorie „körperliche Beschwerden“ des Kategoriensystems

Die unterschiedlichen Zahlen der absoluten Häufigkeit haben zu t4 und t5 ähnliche Prozentsätze, da die fehlenden Angaben zum Erhebungszeitpunkt t4 am höchsten sind (siehe dazu Angaben unter 3.9).

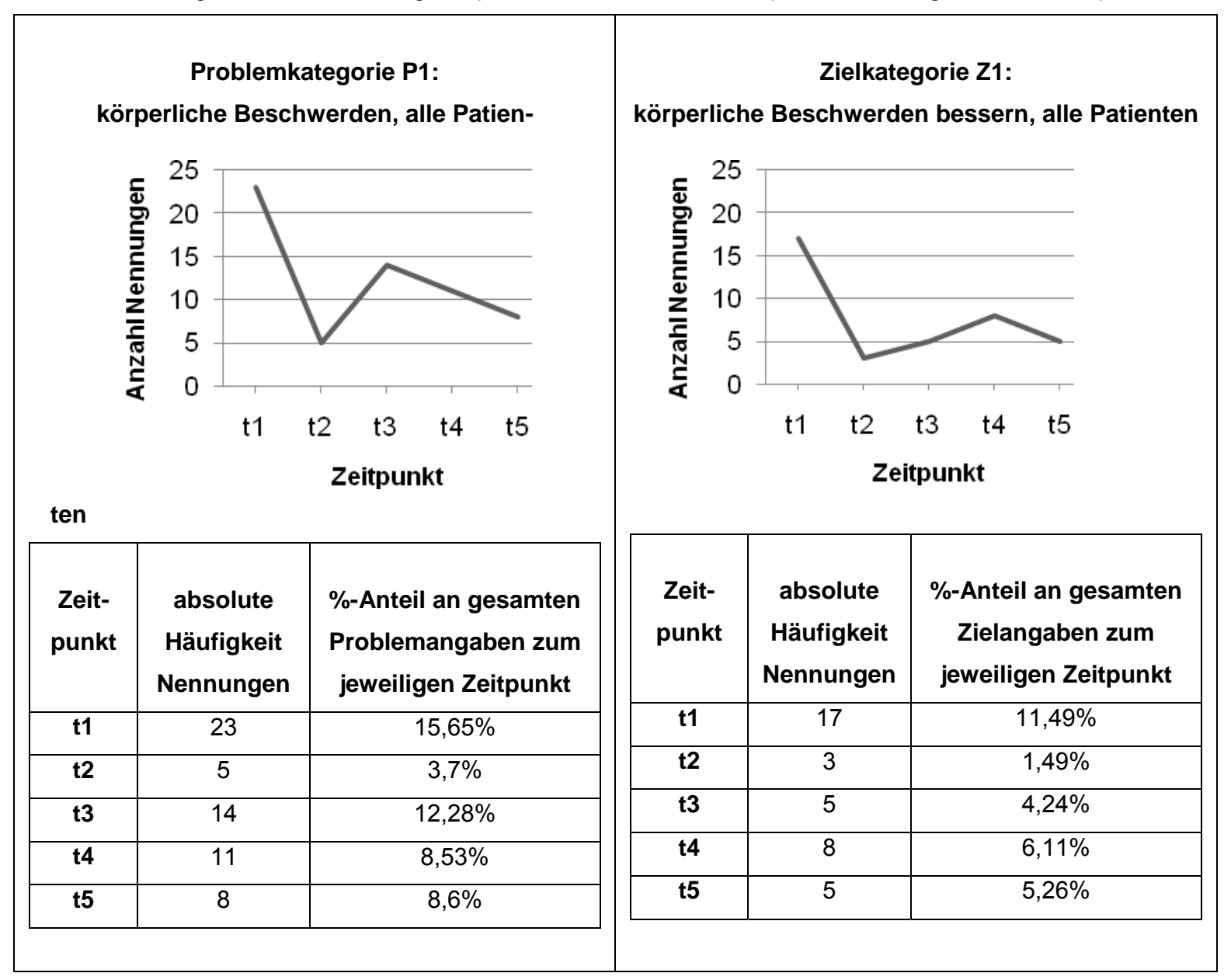

Depressive Beschwerden: 
Einen ähnlichen Verlauf zeigen Probleme der Kategorie „depressive Beschwerden“ (P2), sie gehen insgesamt bis zum Ende der Therapie zurück (danach kurzzeitig leichter Anstieg).

\section{Angst:}

Angst wird im Laufe der Therapie stetig seltener als Problem oder Ziel, Angst zu bewältigen, genannt. Zum Zeitpunkt t1 beträgt der Anteil dieser Kategorie an allen genannten Problemen 32,65\%, an allen angegeben Zielen 27,03\%. Zu Therapieende beinhalten nur noch 19,3\% der Probleme Angst, das Ziel, diese zu bessern wird in $8,47 \%$ der Zielangaben beschrieben. Auch nach Therapieende sinkt der prozentuale Anteil der Angstprobleme weiter, zu t5 beträgt er nur noch 15,05\% von allen genannten Problemen. Die Zielsetzung Angst zu bewältigen steigt nach Therapieende prozentual leicht an, zu t5 liegt der Anteil dieser Kategorie an allen Zielangaben bei $9,47 \%$.

Abbildung 4: zeitliche Entwicklung von Kategorie „Angst“ des Kategoriensystems

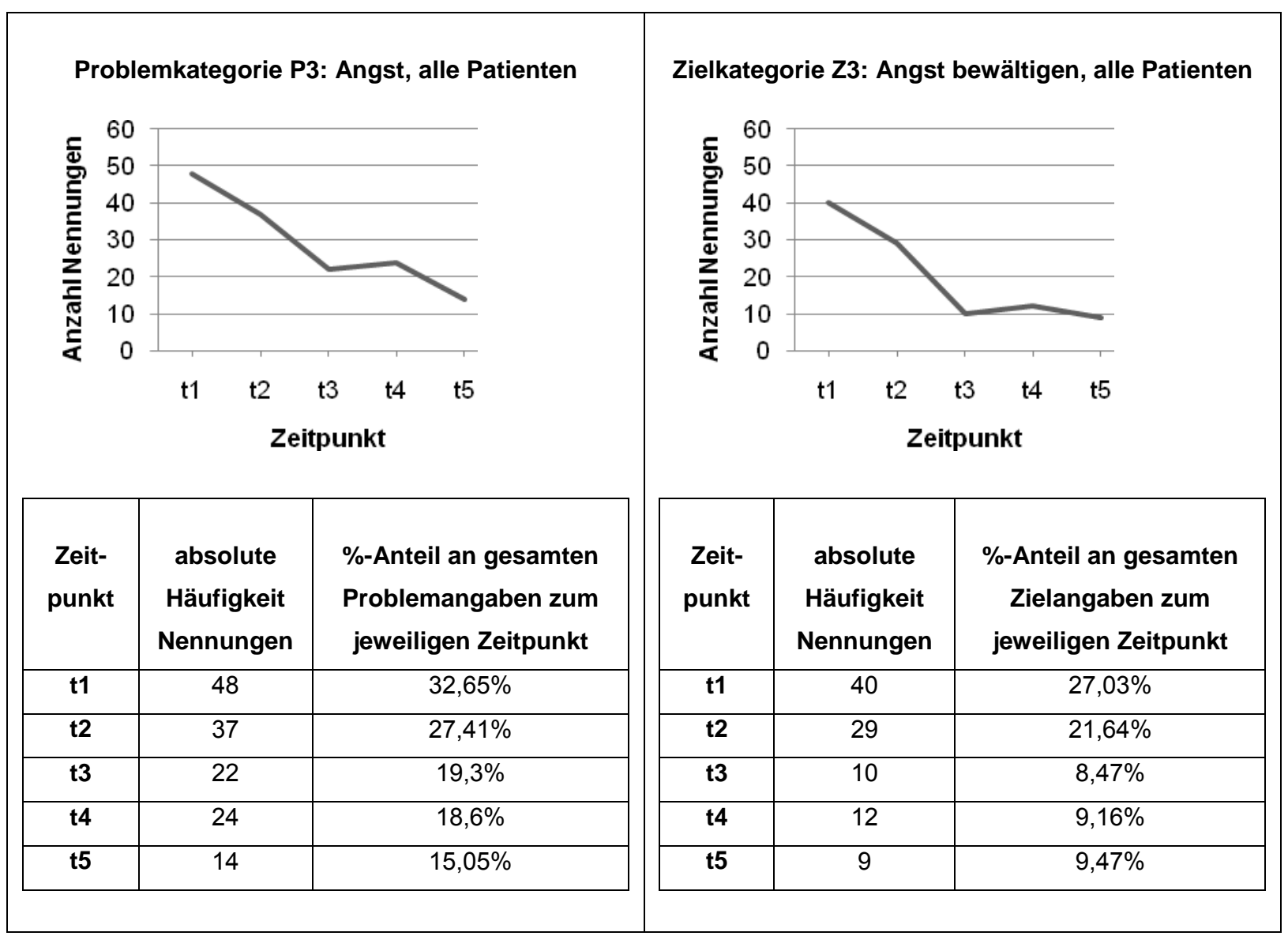


$\underline{\text { Ungelöste Probleme/ Konflikte, z.B. in Alltag oder Beruf und das Ziel, diese Probleme }}$ zu bewältigen:

Analysiert man die Kollektivdaten aller an der Studie teilnehmenden Patienten, ergibt sich von Beginn bis zum Ende der Studie ein Anstieg der Kategorie „ungelöste Probleme/ Konflikte, z.B. Alltag, Beruf" (Kategorie P6). Die Patienten gaben zu Anfang der Behandlung insgesamt seltener derartige Probleme an, der Prozentsatz dieser Kategorie an allen zum Zeitpunkt t1 gemachten Problemangaben beträgt 19,05\%. Am Ende der Therapie (Befragungszeitpunkt $\mathrm{t} 3$ ) liegt dieser Prozentsatz bei $30,7 \%$ und zum Katamnese-Zeitpunkt t5 sogar bei $49,46 \%$. Es kommt folglich bei vielen Patienten zu einer Änderung der Probleminhalte. Mit ähnlichem Verlauf wandeln sich auch die Zielsetzungen dahingehend, Altagsprobleme lösen zu wollen.

Abbildung 5: zeitliche Entwicklung von Kategorie „ungelöste Probleme/ Konflikte, z.B. Alltag und Beruf, Finanzen" des Kategoriensystems

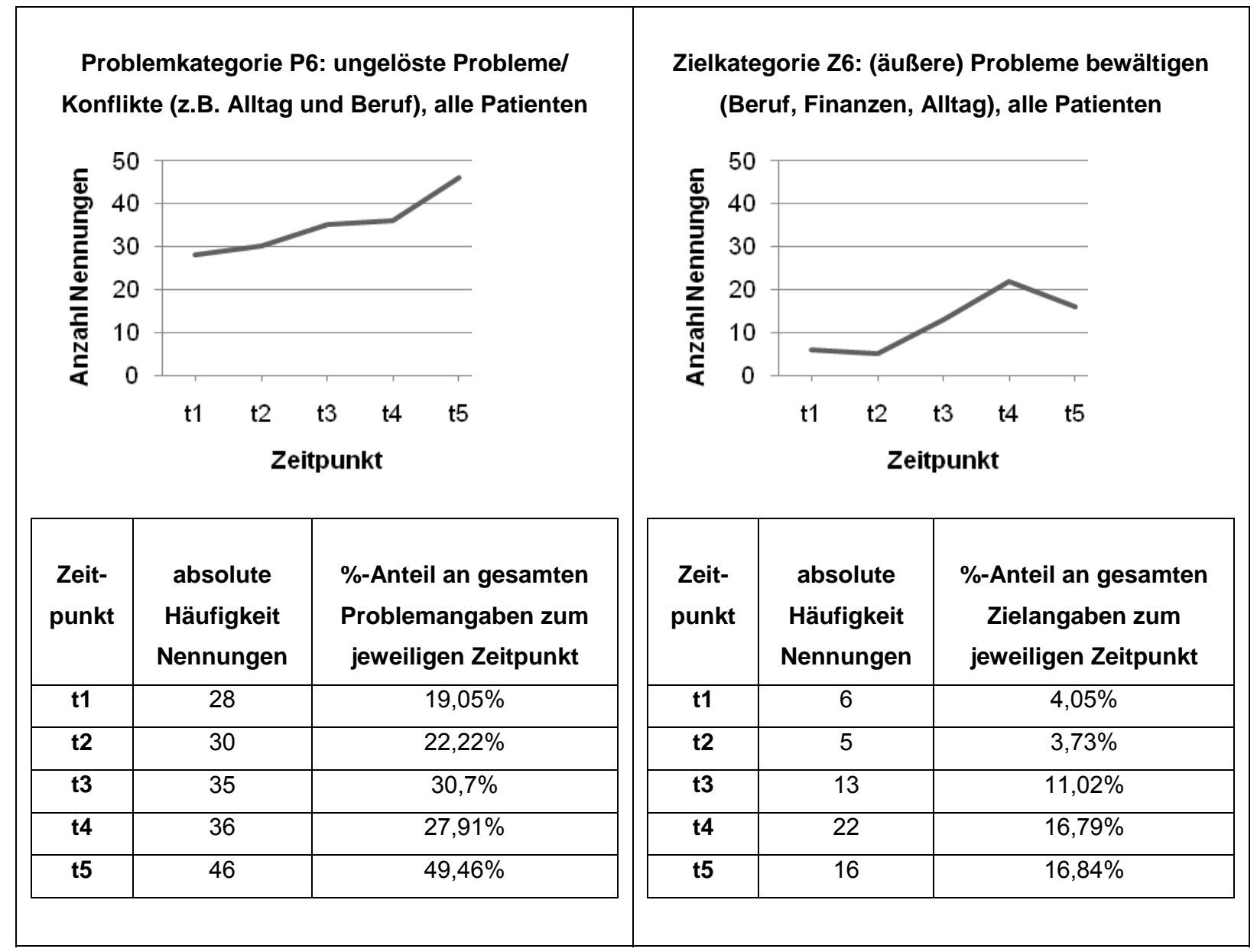

Selbstbezogene Probleme und das Ziel, Ruhe und Gelassenheit zu finden: Aussagen, welche das Ziel enthalten, Ruhe und Gelassenheit zu finden (Kategorie Z7), werden zu Beginn der Psychotherapie insgesamt recht häufig genannt (zu t1 
liegt der Anteil derartiger Probleme bei 35,14\%). In der ersten Hälfte der Behandlung (zwischen $\mathrm{t} 1$ und $\mathrm{t} 2$ ) reduzieren sich solche Aussagen, ebenso nach Therapieende. So liegt ein Jahr nach Beendigung der Behandlung (t5) der Anteil dieser Zielkategorie bei $26,32 \%$.

Probleminhalte dieser Kategorie („sonstige selbstbezogene Probleme“) sind vergleichsweise seltener vorkommend. Im Verlauf der Therapie steigt der prozentuale Anteil dieser Probleme an allen genannten Problemen von $\mathrm{t} 1$ (3. Therapiesitzung) $6,8 \%$ auf $\mathrm{t} 3$ (Therapieende) $11,4 \%$ an.

Abbildung 6: zeitliche Entwicklung von Kategorie „eigene Befindlichkeit“ des Kategoriensystems

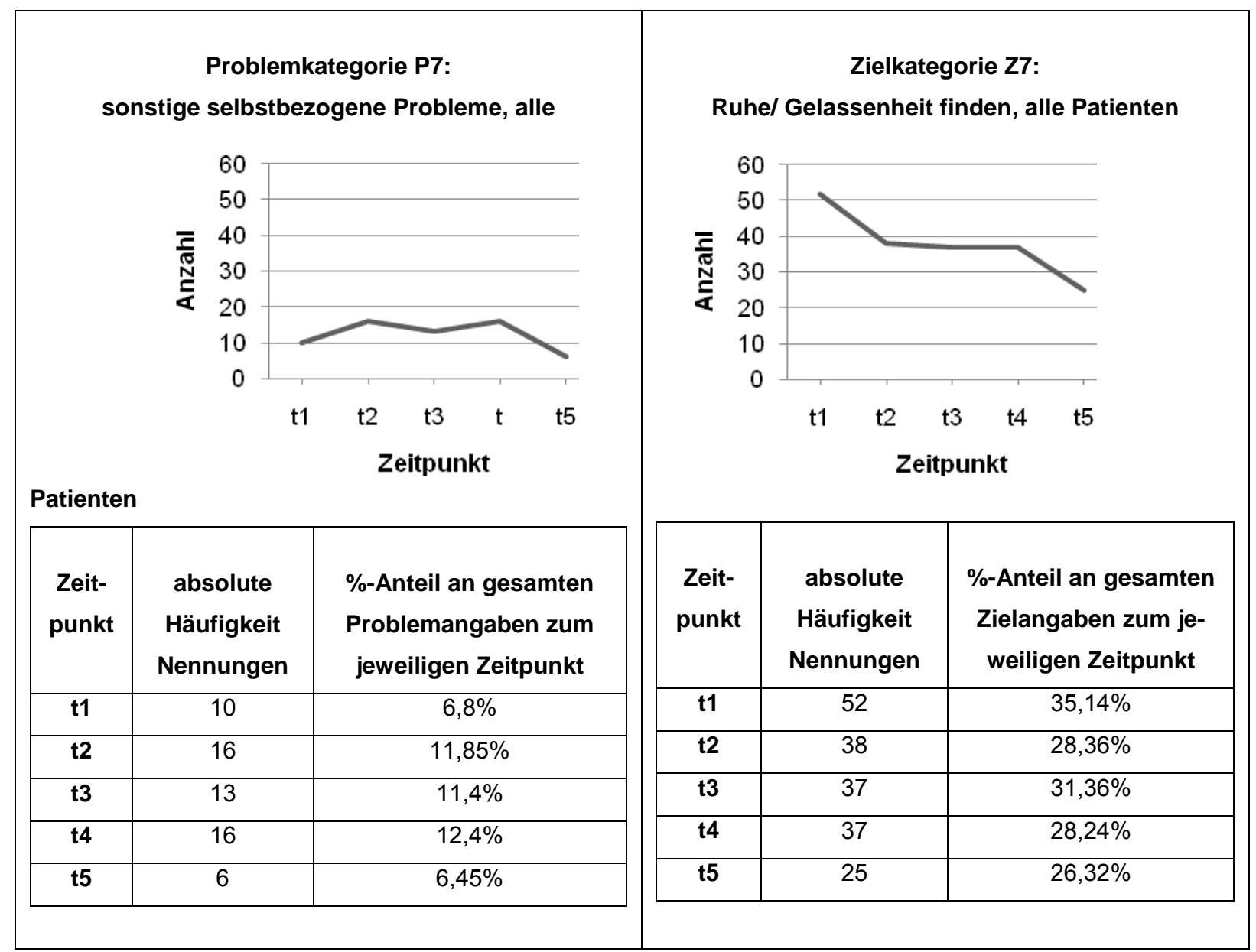

Interpersonelle Probleme:

Interpersonelle Probleme (also Kategorie P8 bzw. Z8 mit den entsprechenden SubKategorien) zeigen insgesamt deutliche Veränderungen in der Anzahl der Nennungen während der Therapie. Insgesamt beträgt ihr Anteil zu t1 6,8\%, zu t3 11,4\% und zu t5 9,68\% von allen genannten Probleme zum jeweiligen Zeitpunkt. 


\section{Sucht/ Essstörung:}

Ein relativ gleichbleibender Verlauf ergibt sich bei der Problemkategorie „Sucht/ Essstörung" (Kategorie P4). Während der Therapie werden dieser Kategorie inhaltlich keine und zum Zeitpunkt t4 nur zwei Probleme zugeordnet.

Freiheiten und Fähigkeiten erlangen:

Ziele, welche der Kategorie „Freiheiten, Fähigkeiten erlangen“ (Kategorie Z9) zugeordnet wurden, nehmen einen peakartigen Verlauf. Nach einem anfänglichen Anstieg wird hier die Höchstzahl zum Zeitpunkt t2, also nach 12 Therapiesitzungen erreicht (t2: 32 Problem Nennungen dieser Kategorie, der Anteil an allen zu t2 genannten Problemen liegt damit bei $23,88 \%$ ).

Abbildung 7: zeitliche Entwicklung von Zielkategorie „Freiheiten, Fähigkeiten erlangen“ des Kategoriensystems

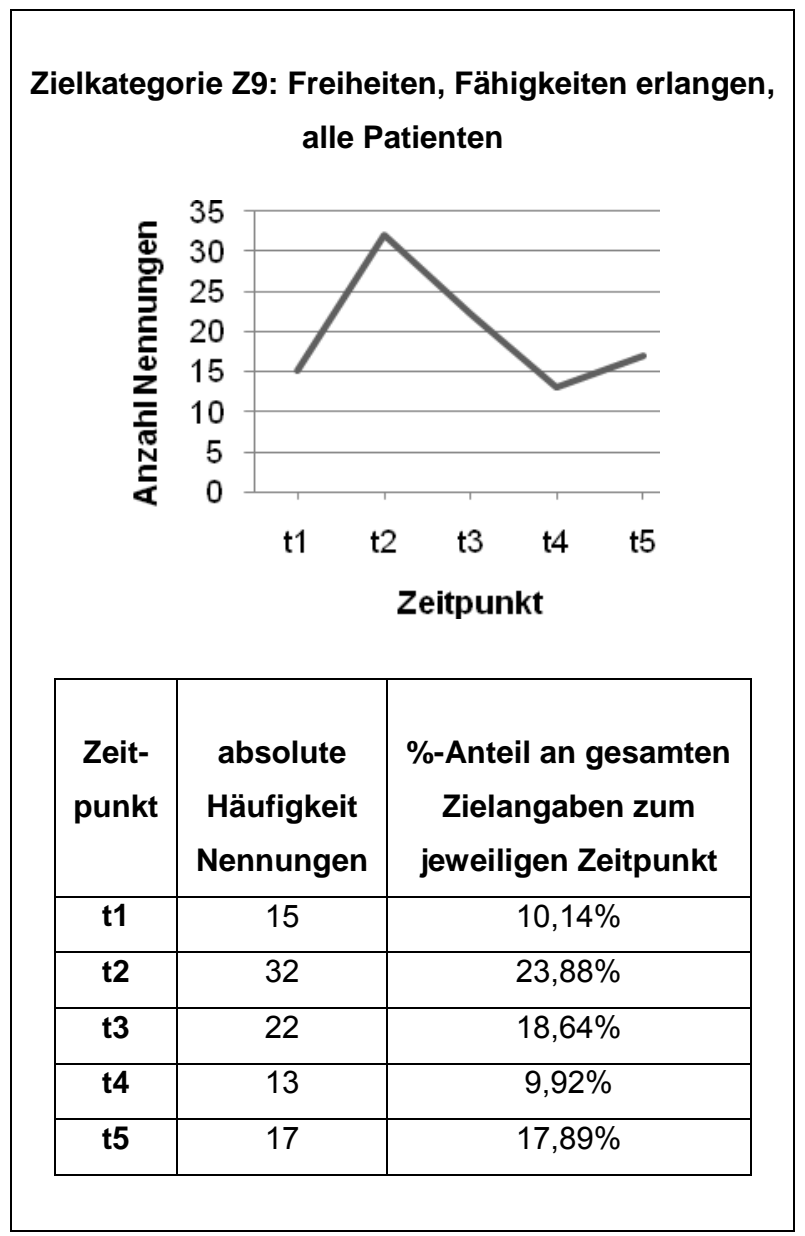

\subsubsection{Verteilung von allen genannten Problemen und Zielen auf Inhaltskatego-} rien des Kategoriensystems, Vergleich Frauen und Männer:

Die Problem- und Zielangaben der Studienteilnehmer wurden zudem getrennt nach beiden Geschlechtern analysiert. Ähnlich der Geschlechterverteilung des 
Krankheitsbildes der GAS beinhaltet die Untersuchungsgruppe (wie zuvor bereits ausgeführt) deutlich mehr weibliche als männliche Patienten. Unter den 51 Patienten sind 41 Frauen und 10 Männer. Besonders wichtig bei der Gegenüberstellung beider Geschlechter bezüglich angegebener Probleme und Ziele ist folglich der Aspekt, nicht nur die absolute Häufigkeit einer Kategorie des jeweiligen Zeitpunktes zu beurteilen, sondern vor allem deren prozentualen Anteil an allen Angaben.

Zur Übersicht dienen erneut Kreisdiagramme verschiedener Befragungszeitpunkte, welche die Verteilung der von Frauen und Männern genannten Probleme und Ziele auf die Inhaltskategorien des Kategoriensystems wiedergeben. Dabei beziehen sich die Angaben auf alle zum betrachteten Zeitpunkt jeweils von Frauen oder Männern genannten Probleme oder Ziele als 100\%.

Abbildung 8: Verteilung zu t1 und t5 genannter Probleme auf Inhaltskategorien des Kategoriensystems, Frauen und Männer getrennt
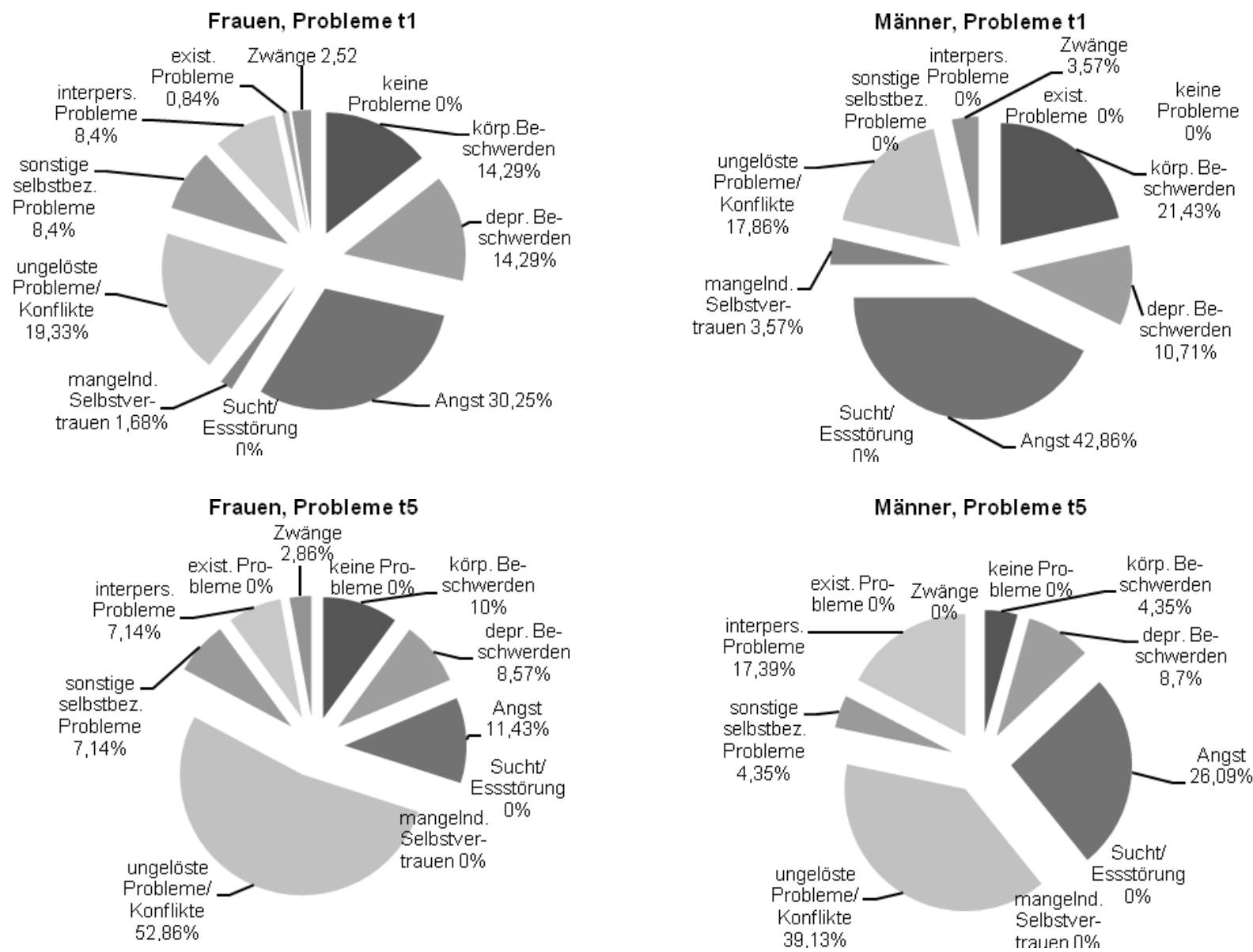
Abbildung 9: Verteilung zu t1 und t5 genannter Ziele auf Inhaltskategorien des Kategoriensystems,

Frauen und Männer getrennt
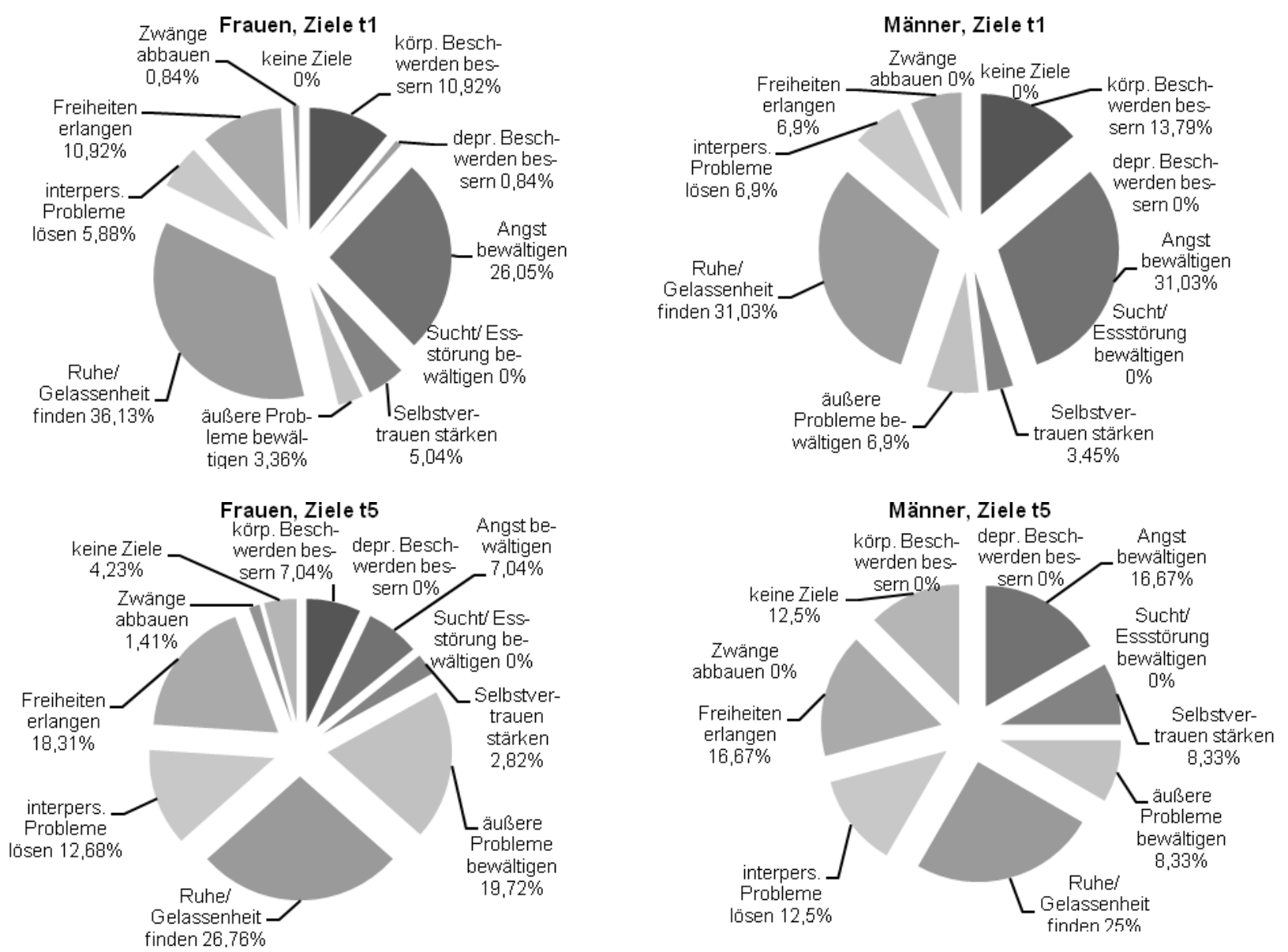

Kreisdiagramme der Zeitpunkte t2 bis t4 befinden sich im Anhang dieser Arbeit unter 7.1.2.

\section{Angst:}

Kategorie „Angst“ (P3) wird zum Befragungszeitpunkt t1 (3. Therapiesitzung) von weiblichen und männlichen Studienteilnehmern häufig genannt. Der Anteil dieser Kategorie an allen Problemaussagen des jeweiligen Geschlechts beträgt zu t1 bei den Frauen $30,25 \%$ und bei den Männern 42,86\%. Im weiteren Verlauf der Therapie geht dieser Prozentsatz bei den Frauen zurück, so dass zu Therapieende (Befragungszeitpunkt t3) nur noch 16,48\% der angegeben Probleme der Patientinnen Angst beinhalten. Bei den Männern wird die Angstproblematik im 
Rahmen der Therapie ebenfalls reduziert, allerdings kommt es zwischen Zeitpunkt t2 und $\mathrm{t3}$ (also zwischen 12. Sitzung und Ende der Therapie) zu einem prozentualen Anstieg auf 30,43\% der von den männlichen Patienten angegebenen Probleme. Hier ist zwar die Anzahl der Problemnennungen, welche Angst beinhalten, von t2 und t3 mit sieben identisch, da jedoch die Anzahl fehlender Angaben steigt, ist der Prozentsatz an den gemachten Aussagen zu t3 entsprechend höher. Unmittelbar posttherapeutisch (also zwischen t3 und t4) sinkt bei den Männern der Prozentsatz angegebener Angstprobleme, während es bei den Frauen zu einem leichten Anstieg kommt. Ein halbes Jahr nach Therapieende kommt es dann bei den Männern ebenfalls zu einem prozentualen Anstieg dieser Kategorie, während bei den Frauen ein Rückgang zu verzeichnen ist.

Abbildung 10: zeitliche Entwicklung von Problemkategorie „Angst“ des Kategoriensystems,

\section{Vergleich Frauen und Männer}

Aufgrund des deutlichen Unterschiedes der Größe beider Vergleichsgruppen (41 Frauen, 10 Männer) ist in dieser Abbildung ein zusätzliches Diagramm zur Darstellung des Prozentsatzes von Angst-Probleme an allen Problemangaben teilnehmender Frauen oder Männer zum jeweiligen Zeitpunkt aufgeführt.

Problemkategorie P3: absolute Häufigkeit Problem

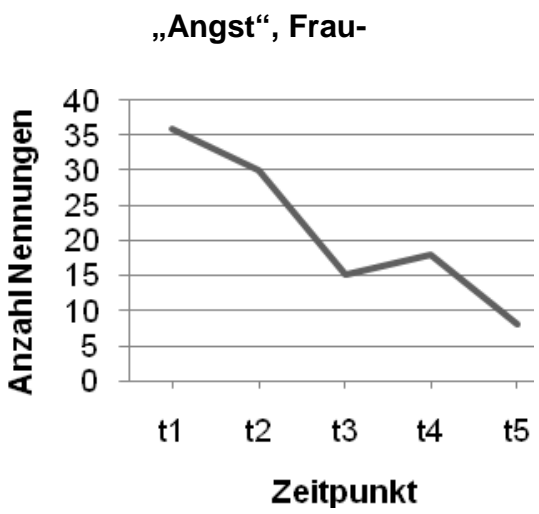

en

Problemkategorie P3: Prozentsatz Problem „Angst“, Frauen

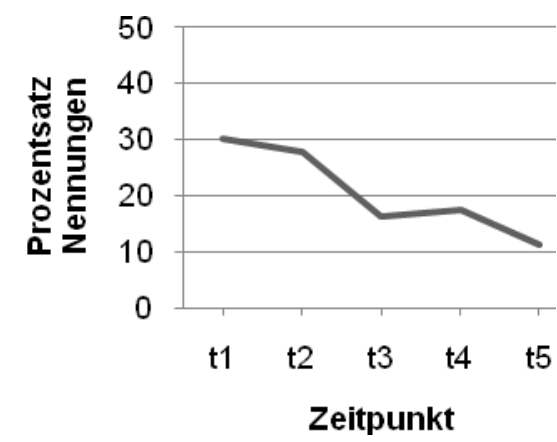

Problemkategorie P3: absolute Häufigkeit Problem „Angst“, Män-

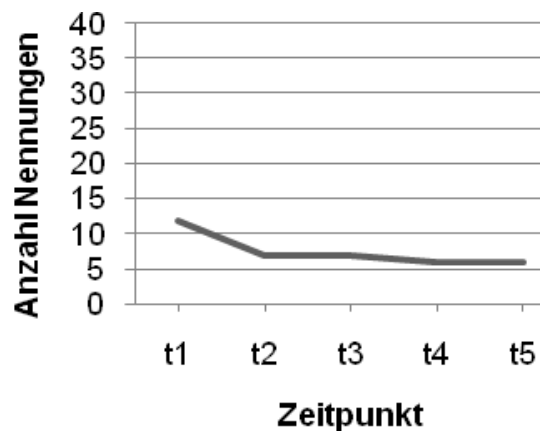

ner

Problemkategorie P3: Prozentsatz Problem „Angst", Männer

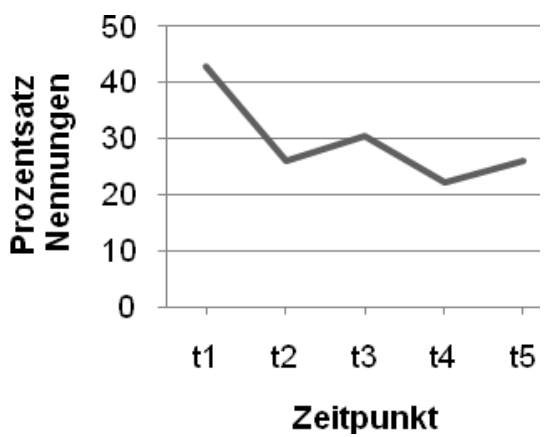




\begin{tabular}{|c|c|c|c|c|c|}
\hline $\begin{array}{l}\text { Zeit- } \\
\text { punkt }\end{array}$ & $\begin{array}{c}\text { absolute } \\
\text { Häufigkeit } \\
\text { Nennungen }\end{array}$ & $\begin{array}{l}\text { \%-Anteil an gesamten } \\
\text { Problemangaben der } \\
\text { weiblichen Patienten } \\
\text { zum jeweiligen Zeitpunkt }\end{array}$ & $\begin{array}{l}\text { Zeit- } \\
\text { punkt }\end{array}$ & $\begin{array}{c}\text { absolute } \\
\text { Häufigkeit } \\
\text { Nennungen }\end{array}$ & $\begin{array}{l}\text { \%-Anteil an gesamten } \\
\text { Problemangaben der } \\
\text { männlichen Patienten } \\
\text { zum jeweiligen Zeitpunkt }\end{array}$ \\
\hline t1 & 36 & $30,25 \%$ & t1 & 12 & $42,86 \%$ \\
\hline t2 & 30 & $27,78 \%$ & t2 & 7 & $25,93 \%$ \\
\hline t3 & 15 & $16,48 \%$ & t3 & 7 & $30,43 \%$ \\
\hline t4 & 18 & $17,65 \%$ & t4 & 6 & $22,22 \%$ \\
\hline t5 & 8 & $11,43 \%$ & t5 & 6 & $26,09 \%$ \\
\hline
\end{tabular}

Körperliche Beschwerden und das Ziel, diese zu bessern:

Zum Zeitpunkt t1 (3. Therapiesitzung) liegt der Anteil von Problemen mit körperlichen Beschwerden (Kategorie P1) an allen von den männlichen Patienten angegebenen Problemen bei 21,43\%, zu t2 (12. Therapiesitzung) bei 7,41\% und am Ende der Therapie, also zum Zeitpunkt $\mathrm{t}$, bei 8,7\%. Insgesamt wurden im Laufe der Therapie folglich die Angaben von körperlichen Beschwerden reduziert. Einschränkend muss jedoch die geringe Anzahl dieser Probleme aufgrund der wenigen männlichen Studienteilnehmer berücksichtigt werden.

Bei den Frauen macht zum Zeitpunkt t1 (3. Therapiesitzung) diese Kategorie 14,29\% der von ihnen genannten Probleme aus und zu t2 schließlich nur noch 2,78\%. Zwischen t2 und Therapieende kommt es zu einem Anstieg, so dass zu t3 13,19\% der von Frauen angegebenen Probleme körperliche Beschwerden beinhalten.

Nach der Therapie sinkt der prozentuale Anteil körperlicher Beschwerden an allen genannten Problemen bei den Männern, während er zwischen t4 und t5 (also zwischen dem 6. und 12. Monat nach der Behandlung) aufgrund der zunehmenden Zahl fehlender Angaben bei den Frauen leicht ansteigt (absolute Anzahl der Probleme sinkt bei den Frauen zwischen $t 4$ und t5).

Abbildung 11: zeitliche Entwicklung von Problemkategorie „körperliche Beschwerden“ des Kategoriensystems, Vergleich Frauen und Männer

Aufgrund des deutlichen Unterschiedes der Größe beider Vergleichsgruppen (41 Frauen, 10 Männer) ist in dieser Abbildung ein zusätzliches Diagramm zur Darstellung des Prozentsatzes von körperlicher Beschwerden an allen Problemangaben teilnehmender Frauen oder Männer zum jeweiligen Zeitpunkt aufgeführt. 


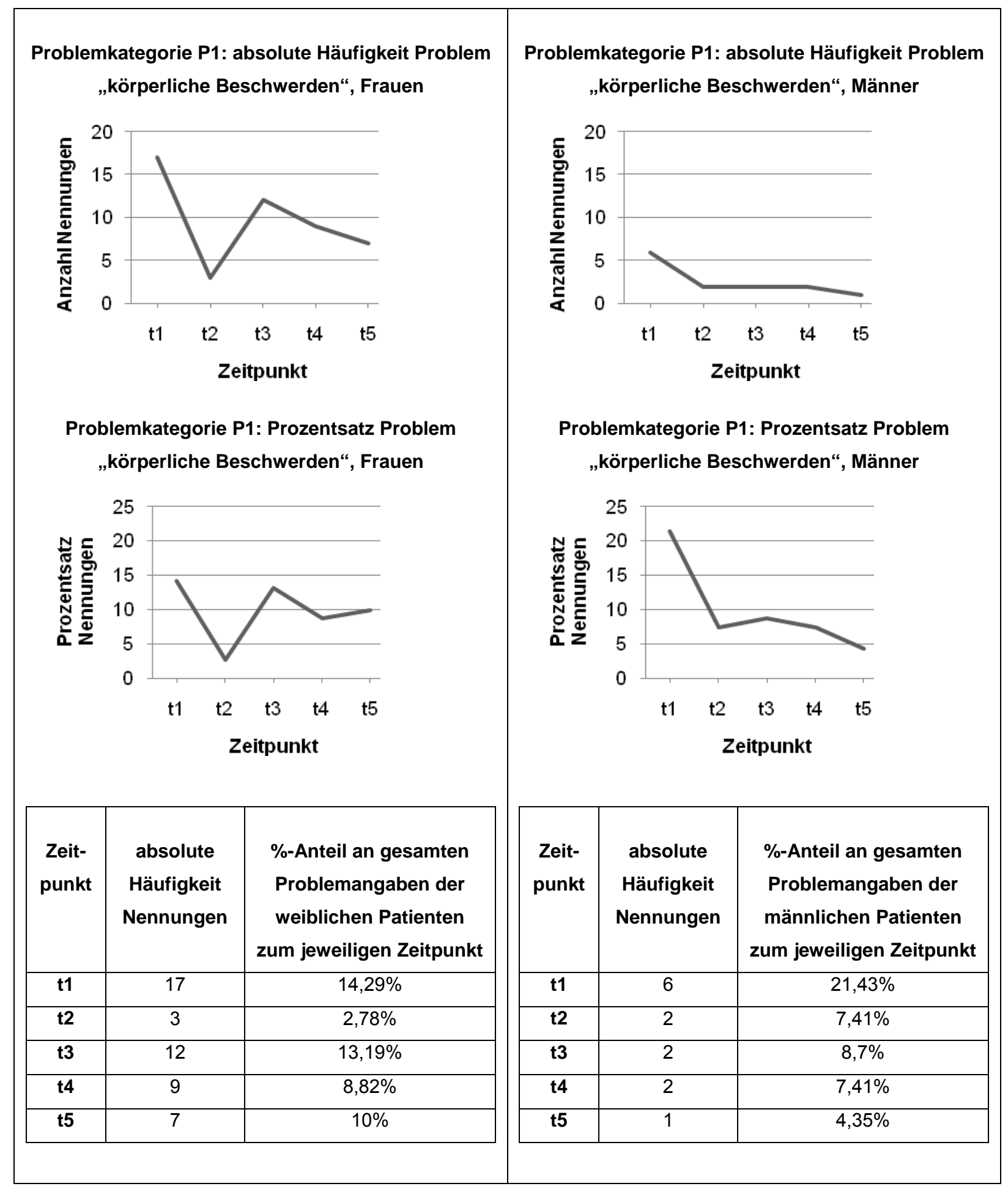

Die Abnahme des prozentualen Anteils der Zielangaben, körperliche Beschwerden zu bessern (Z1), ist bei beiden Geschlechtern zwischen $\mathrm{t} 1 \mathrm{und} \mathrm{t} 2 \mathrm{am}$ deutlichsten.

Abbildung 12: zeitliche Entwicklung von Zielkategorie „körperliche Beschwerden bessern“ des Kategoriensystems, Vergleich Frauen und Männer

Aufgrund des deutlichen Unterschiedes der Größe beider Vergleichsgruppen (41 Frauen, 10 Männer) ist in dieser Abbildung ein zusätzliches Diagramm zur Darstellung des Prozentsatzes von Zielen, körperliche Beschwerden zu bessern an allen Zielangaben teilnehmender Frauen oder Männer zum jeweiligen Zeitpunkt aufgeführt. 


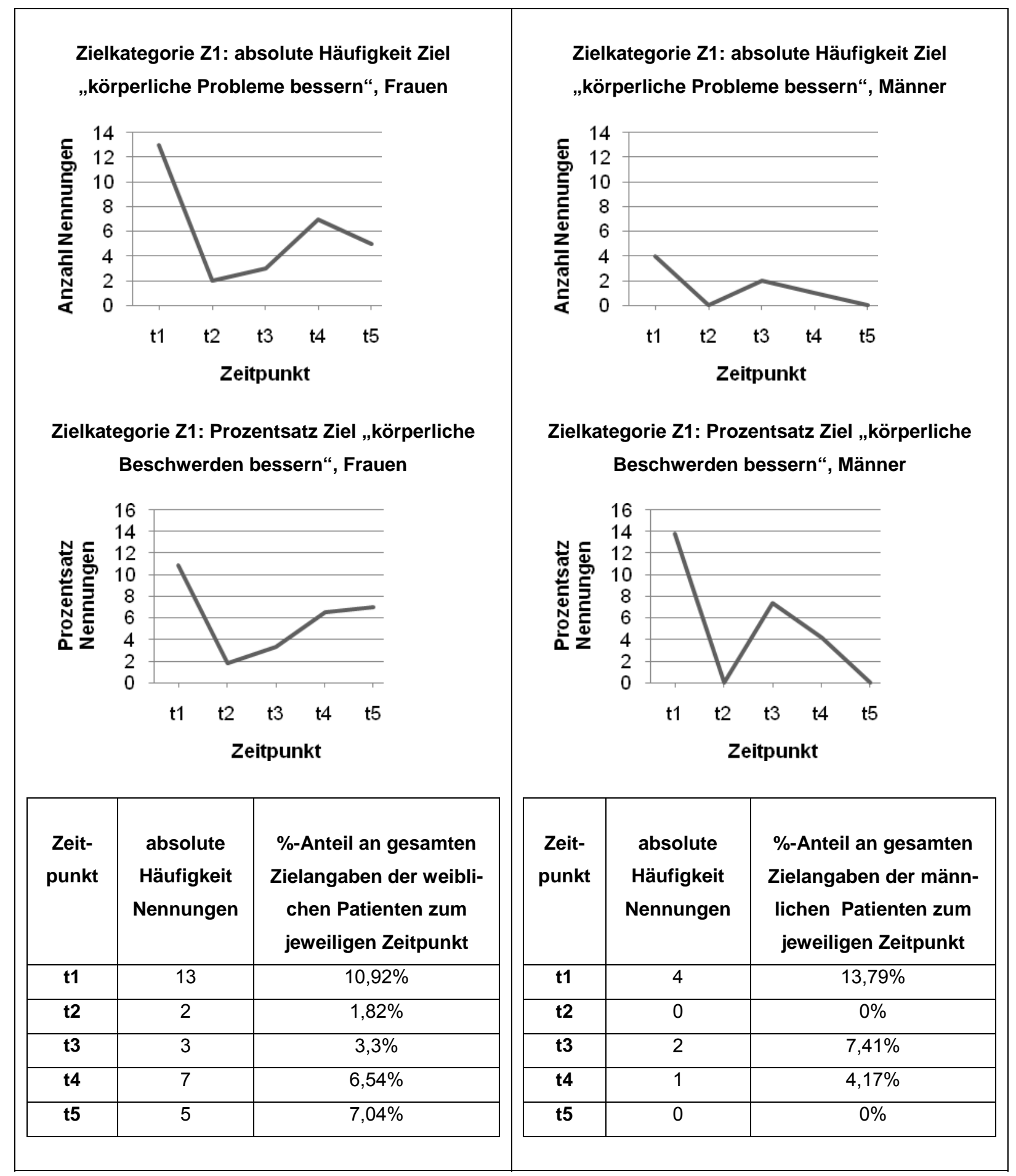

Fazit:

Die Ergebnisse der Prüfung dieser Hypothese sind rein deskriptiv und somit ohne Signifikanztest dargestellt, es zeigen sich jedoch deutliche Veränderungen im Sinne der Hypothese, da sich Probleme und Ziele im in allen Phasen des Therapieverlaufs inhaltlich verändern. 


\subsection{Prüfung von Hypothese $\mathrm{H} 1.2$}

„Die behandelten Patienten formulieren unterschiedliche Probleme und Ziele zu Befragungszeitpunkt $\mathrm{t} 1$, je nachdem, ob sie verhaltenstherapeutisch oder psychodynamisch behandelt werden. Die Patienten wurden zwar randomisiert den Therapieverfahren zugewiesen, jedoch fanden vor Zeitpunkt $\mathrm{t} 1$ bereits drei diagnostische und therapeutische Sitzungen statt."

Insgesamt haben 51 Patienten an der Studie teilgenommen, von denen 26 verhaltenstherapeutisch und 25 psychodynamisch behandelt wurden.

Auch bei der vorliegenden Hypothese fand die Auswertung mit Hilfe des Kategoriensystems statt. Inhaltliche Unterschiede von Problemen oder Zielen können mit diesem Hilfsmittel am besten festgestellt werden, wobei für die Hypothesen Überprüfung H1.2 der Fokus auf Therapiezeitpunkt t1 (3. Therapiesitzung) gesetzt wurde. Zu diesem Zeitpunkt der Befragung fanden bereits in drei Therapiestunden Gespräche mit dem behandelnden Psychotherapeuten statt.

Die Ergebnisse des Ratings der zum Erhebungszeitpunkt $\mathrm{t} 1$ angegebenen Probleme und Ziele anhand des Kategoriensystems sind in den folgenenden Kreisdiagrammen getrennt nach Therapieverfahren dargestellt. Es werden alle zum Zeitpunkt t1 vorhandenen Problem- und Zielaussagen einer Therapiegruppe als 100\% gewertet, der Prozentsatz einer Kategorie bezieht sich also auf den Anteil an den abgegebenen Problem- und Zielaussagen der Therapiegruppe zu t1. Aussagen "keine Probleme“ oder "keine Ziele“ zu haben wurde zum Befragungszeitpunkt t1 nicht genannt. Hierbei handelt es sich um einzelne Angaben von Patienten zu den Befragungszeitpunkten $\mathrm{t} 2$ bis $\mathrm{t} 5$, welche ausdrücken, momentan kein Problem oder Ziel zu haben. Davon abzugrenzen sind fehlende Aussagen (nähere Angaben unter 3.9). 
Abbildung 13: Verteilung der zu Befragungszeitpunkt t1 angegebenen Probleme und Ziele auf Inhaltskategorien des Kategoriensystems, Vergleich der Therapiegruppen

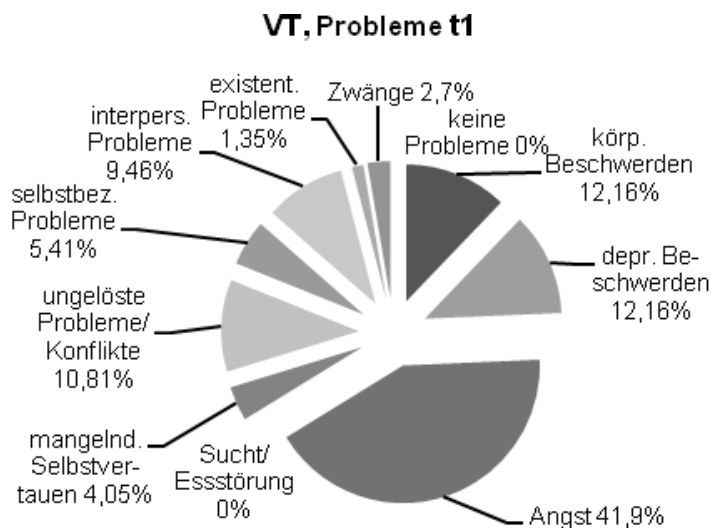

VT, Ziele t1

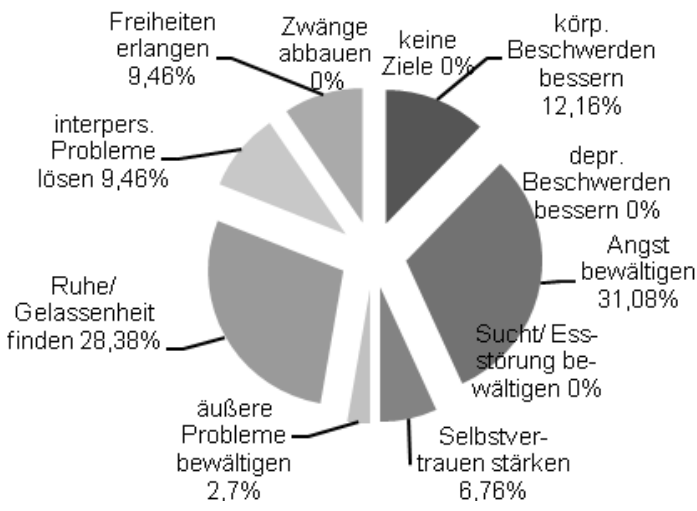

SET, Probleme t1
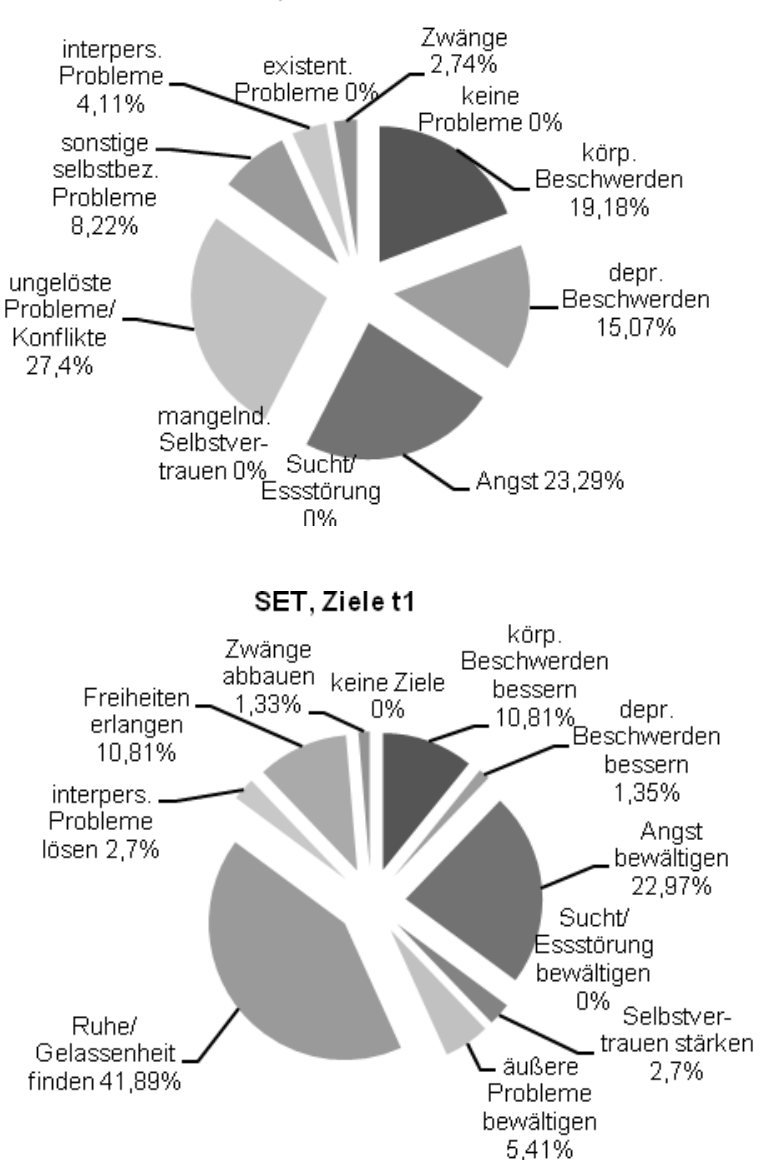

In den nächsten Unterpunkten werden einzelne Kategorien nach Therapiegruppen separat analysiert und ihre absoluten Häufigkeiten zum Zeitpunkt t1 dargestellt.

\section{Körperliche Beschwerden:}

Betrachtet man die Kategorie „körperliche Beschwerden“ (Kategorie P1) des Kategoriensystems, so fällt auf, dass zur SET-Gruppe randomisierte Patienten zu Beginn der Behandlung (Erhebungszeitpunkt t1) häufiger Probleminhalte dieser Kategorie angeben.

Tabelle 5: Verteilung von Problemkategorie 1 des Kategoriensystems („,körperliche Beschwerden“) zum Zeitpunkt t1, Vergleich der Therapiegruppen

Problemkategorie 1 „körperliche Beschwerden“ zum Zeitpunkt t1

\begin{tabular}{|c|c|c|}
\hline & VT & SET \\
\hline Absolute Häufigkeit Problemnennungen & 9 & 14 \\
\hline
\end{tabular}




\begin{tabular}{|c|c|c|}
\hline $\begin{array}{c}\text { \%-Anteil an gesamten Problemangaben der } \\
\text { Patienten einer Therapiegruppe zu t1 }\end{array}$ & $12,16 \%$ & $19,18 \%$ \\
\hline
\end{tabular}

Die Zielangaben der Patienten, welche körperliche Beschwerden zu bessern beinhalten (Kategorie Z1), sind zu t1 unter beiden Therapieformen prozentual nahezu identisch vertreten (VT 12,16\%, SET 10,81\% der Zielangaben ihrer Behandlungsgruppe).

\section{Angst:}

Kategorie „Angst" (Kategorie P3), bei welcher die Patienten in den Formulierungen explizit Angst angeben, macht zum Befragungszeitpunkt $\mathrm{t} 1$ in der Gruppe der verhaltenstherapeutisch behandelten Personen 41,9\% der Problemnennungen aus, während in der SET-Gruppe nur 23,29\% der Probleme dieser Kategorie zugeordnet werden konnten.

Entsprechend beinhalten zum Erhebungszeitpunkt t1 unter VT 31,08\% der Zielangaben Aussagen, Angst bewältigen zu wollen. Unter psychodynamischer Behandlung beträgt dieser Prozentsatz nur 22,97\%.

Tabelle 6: Verteilung von Problem- und Zielkategorie 3 des Kategoriensystems („Angst“) zum Zeitpunkt t1, Vergleich der Therapiegruppen

\begin{tabular}{|c|c|c|c|c|}
\hline \multicolumn{3}{|c|}{ Kategorie 3 zum Zeitpunkt t1 } \\
\hline & \multicolumn{2}{|c|}{ VT } & \multicolumn{2}{c|}{ SET } \\
\cline { 2 - 5 } & $\begin{array}{c}\text { Problem } \\
\text { „Angst“ }\end{array}$ & $\begin{array}{c}\text { Ziel „Angst „Angst } \\
\text { bewältigen“ }\end{array}$ & $\begin{array}{c}\text { Problem } \text { "Angst“ } \\
\text { bewältigen“ }\end{array}$ & 17 \\
\hline $\begin{array}{c}\text { Absolute Häufigkeit Problem- oder } \\
\text { Zielnennungen der Kategorie }\end{array}$ & 31 & 23 & 17 & $22,97 \%$ \\
\hline $\begin{array}{c}\text { \%-Anteil an gesamten Problem- oder } \\
\text { Zielangaben der Patienten einer } \\
\text { Therapiegruppe zu t1 }\end{array}$ & $41,9 \%$ & $31,08 \%$ & $23,29 \%$ & \\
\end{tabular}

Ungelöste Probleme und Konflikte sowie das Ziel, diese zu bewältigen:

Auch in Kategorie "ungelöste Probleme/ Konflikte, z.B. Altag und Beruf" (Kategorie P6 des Kategoriensystems) fallen Unterschiede der Probleme zum Zeitpunkt der 3. Therapiesitzung (t1) auf. Demnach machen Patienten der SET-Gruppe häufiger derartige Angaben (SET 27,4\%, VT 10,81\% aller Problemangaben der jeweiligen Therapiegruppe zu t1). In der Anzahl der Ziele dieser Kategorie („(äußere) Probleme bewältigen") unterscheiden sich beide Therapieformen ebenfalls voneinander. Jedoch 
werden solche Ziele insgesamt seltener als Probleme dieser Kategorie angegeben (SET 5,41\%, VT 2,7\% aller Zielangaben ihrer Therapiegruppe zu t1).

Tabelle 7: Verteilung von Problem- und Zielkategorie 6 des Kategoriensystems („ungelöste Problemel Konflikte“) zum Zeitpunkt t1, Vergleich der Therapiegruppen

\begin{tabular}{|c|c|c|c|c|}
\hline \multicolumn{5}{|c|}{ Kategorie 6 zum Zeitpunkt t1 } \\
\hline & \multicolumn{2}{|c|}{ VT } & \multicolumn{2}{|c|}{ SET } \\
\hline & $\begin{array}{l}\text { Problem } \\
\text { „ungelöste } \\
\text { Probleme“ }\end{array}$ & $\begin{array}{l}\text { Ziel „(äußere) } \\
\text { Probleme } \\
\text { bewältigen“ }\end{array}$ & $\begin{array}{l}\text { Problem } \\
\text { „ungelöste } \\
\text { Probleme“ }\end{array}$ & $\begin{array}{c}\text { Ziel „(äußere) } \\
\text { Probleme } \\
\text { bewältigen“ }\end{array}$ \\
\hline $\begin{array}{l}\text { Absolute Häufigkeit Problem- oder } \\
\text { Zielnennungen der Kategorie }\end{array}$ & 8 & 2 & 20 & 4 \\
\hline $\begin{array}{c}\text { \%-Anteil an gesamten Problem- oder } \\
\text { Zielangaben der Patienten einer } \\
\text { Therapiegruppe zu t1 }\end{array}$ & $10,81 \%$ & $2,7 \%$ & $27,4 \%$ & $5,41 \%$ \\
\hline
\end{tabular}

Sonstige selbstbezogene Probleme und das Ziel, Ruhe und Gelassenheit zu finden: Probleme, welche inhaltlich der Kategorie „sonstige selbstbezogene Probleme“ (Kategorie P7) zugeordnet wurden, zeigen eine ähnliche Verteilung in beiden Therapieformen.

Die Angaben, welche Ziele der Kategorie „Ruhe und Gelassenheit finden“ (Kategorie Z7) enthalten, unterscheiden sich in den Anzahlen zwischen beiden verglichenen Therapieorientierungen: Die SET-Patienten geben Ziele dieser Kategorie demnach zu Therapiebeginn häufiger an (41,89\% der Zielangaben bei SET, 28,38\% bei VT).

Tabelle 8: Verteilung von Zielkategorie 7 des Kategoriensystems („Ruhe und Gelassenheit finden“) zum Zeitpunkt t1, Vergleich der Therapiegruppen

Anhand dieser Tabelle wird erneut deutlich, dass jeder Patient nach drei Zielen gefragt wurde, da in der SETGruppe mehr Probleme dieser Kategorie als Patienten vorliegen (31 Probleme dieser Kategorie wurden von einer Gruppe aus 25 SET-Patienten genannt)

\begin{tabular}{|c|c|c|}
\hline \multicolumn{2}{|c|}{ Zielkategorie 7 „Ruhe und Gelassenheit finden“ zum Zeitpunkt t1 } \\
\hline & VT & SET \\
\hline Absolute Häufigkeit Zielnennungen der Kategorie & 21 & $31,89 \%$ \\
\hline $\begin{array}{c}\text { \%-Anteil an gesamten Zielangaben der Patienten } \\
\text { einer Therapiegruppe zu t1 }\end{array}$ & $28,38 \%$ & \\
\hline
\end{tabular}


Interpersonelle Probleme und das Ziel, diese zu lösen:

"Interpersonelle Probleme“ der Kategorie P8 (Sub-Kategorien 8k, 8d, 8s und 8x zusammengefasst) werden von VT-Patienten zu t1 häufiger angegeben. Der Anteil an allen Problemangaben der Therapiegruppe zu diesem Befragungszeitpunkt beträgt hier $9,46 \%$, zeitgleich in der SET-Gruppe 4,11\%. Ziele der Kategorie „interpersonelle Probleme lösen" (Z8) werden ebenfalls häufiger von Patienten der VT-Gruppe angegeben $(9,46 \%$ aller Zielaussagen bei VT und $2,7 \%$ bei SET).

Tabelle 9: Verteilung von Problem- und Zielkategorie 8 des Kategoriensystems

(„interpersonelle Probleme“) zum Zeitpunkt t1, Vergleich der Therapiegruppen

\begin{tabular}{|c|c|c|c|c|}
\hline \multicolumn{2}{|c|}{ Kategorie 8 zum Zeitpunkt t1, Gesamtzahlen (Sub-Kategorien k, d, s und x summier) } \\
\hline \multirow{2}{*}{$\begin{array}{c}\text { Problem „inter- } \\
\text { personelle } \\
\text { Probleme“ }\end{array}$} & $\begin{array}{c}\text { Ziel „,interper- } \\
\text { sonelle Prob- } \\
\text { leme lösen“ }\end{array}$ & $\begin{array}{c}\text { Problem „inter- } \\
\text { personelle Prob- } \\
\text { leme“ }\end{array}$ & $\begin{array}{c}\text { Ziel „interper- } \\
\text { sonelle Proble- } \\
\text { me lösen“ }\end{array}$ \\
\hline $\begin{array}{c}\text { Absolute Häufigkeit Problem- oder } \\
\text { Zielnennungen der Kategorie }\end{array}$ & 7 & 7 & 3 & 2 \\
\hline $\begin{array}{c}\text { \%-Anteil an gesamten Problem- } \\
\text { oder Zielangaben der Patienten } \\
\text { einer Therapiegruppe zu t1 }\end{array}$ & $9,46 \%$ & $9,46 \%$ & $4,11 \%$ & $2,7 \%$ \\
\hline
\end{tabular}

Freiheiten und Fähigkeiten erlangen:

Der Zielkategorie „Freiheiten, Fähigkeiten erlangen“ (Kategorie Z9) wurden zu Therapiebeginn unter VT sieben Ziele zugewiesen (entspricht 9,46\% aller Zielangaben der VT-Patienten zu t1), unter SET waren es acht solcher Ziele (entspricht 10,81\% der SET-Problemangaben zu t1).

\section{Zwänge:}

Kategogie „Zwänge“ (Kategorie P10, Z10) wird sehr selten genannt, beide Therapieformen unterscheiden sich zu t1 kaum.

\section{Fazit:}

Die vorliegenden Ergebnisse der Hypothesenprüfung wurde im Rahmen dieser Arbeit nicht auf ihre statistische Signifikanz hin überprüft. Deskriptiv weichen zu Therapiebeginn beide verglichenen Behandlungsgruppen hinsichtlich der 
Inhalte genannter Probleme und Ziele oftmals voneinander ab, so dass sich Unterschiede im Sinne der Hypothese zeigen. Erhebliche Abweichungen bestehen demnach z.B. in Angaben von Problemen oder Zielen des Inhaltes „Angst“, „ungelöste Problemel Konflikte“ oder „Ruhe und Gelassenheit finden".

\subsection{Prüfung von Hypothese H1.3}

„Je nach Therapieverfahren (verhaltenstherapeutisch oder psychodynamisch/ SET) werden im Therapieverlauf unterschiedliche Probleme und Ziele genannt.“

Diese Hypothese setzt den Grundgedanken der vorherigen ( $\mathrm{H} 1.2)$ fort, indem Unterschiede der Problem- und Zielangaben beider Therapiegruppen im gesamten Untersuchungszeitraum erfasst werden. Da diese Hypothese mögliche inhaltliche Unterschiede von Problemen und Zielen im Zeitverlauf analysiert, wurde die Auswertung erneut mit dem Kategoriensystem durchgeführt.

Kreisdiagramme der prozentualen Verteilung von Problem- und Zielnennungen beider Therapiegruppen auf Inhaltskategorien können eine erste Übersicht vermitteln. Dabei entsprechen 100\% allen zum jeweiligen Zeitpunkt gemachten Problem- oder Zielangaben einer Therapiegruppe. Die Kreisdiagramme des Zeitpunktes $\mathrm{t} 1$ sind unter 3.4. der Hypothesen Prüfung H1.2 einsehbar, die der Zeitpunkte t2 bis t4 befinden sich im Anhang unter 7.2.

Abbildung 14: Verteilung zu t5 genannter Probleme und Ziele beider Therapiegruppen auf Inhaltskategorien des Kategoriensystems
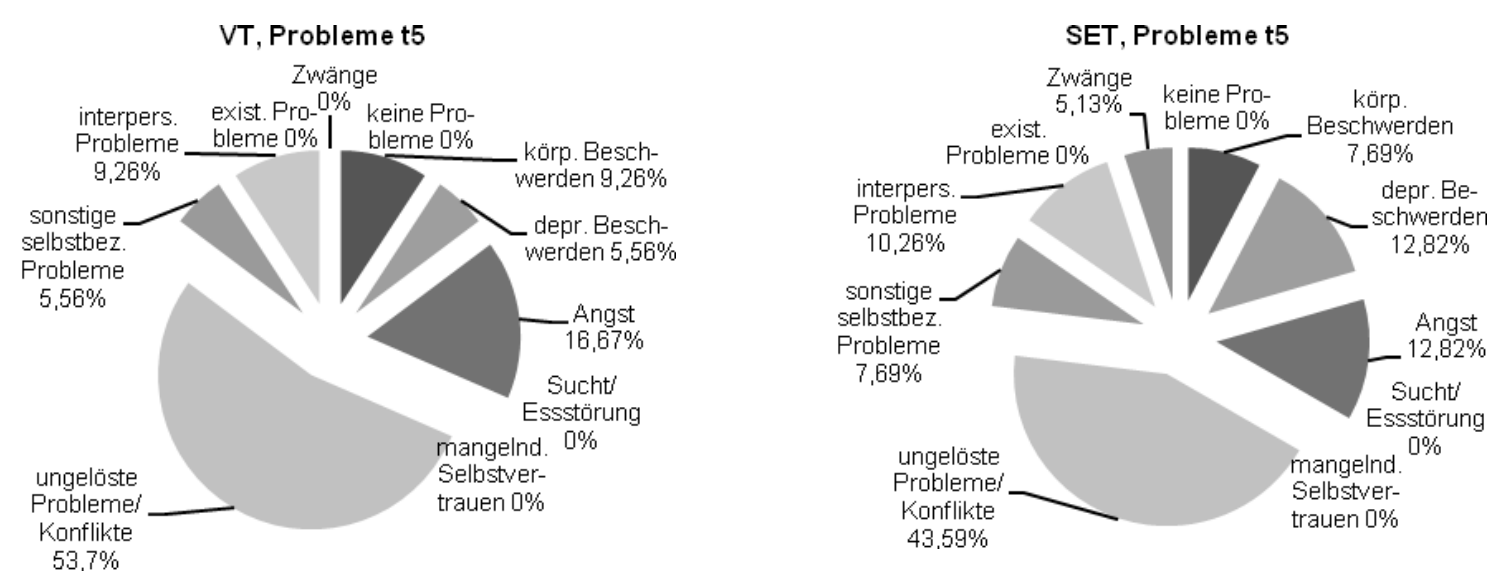

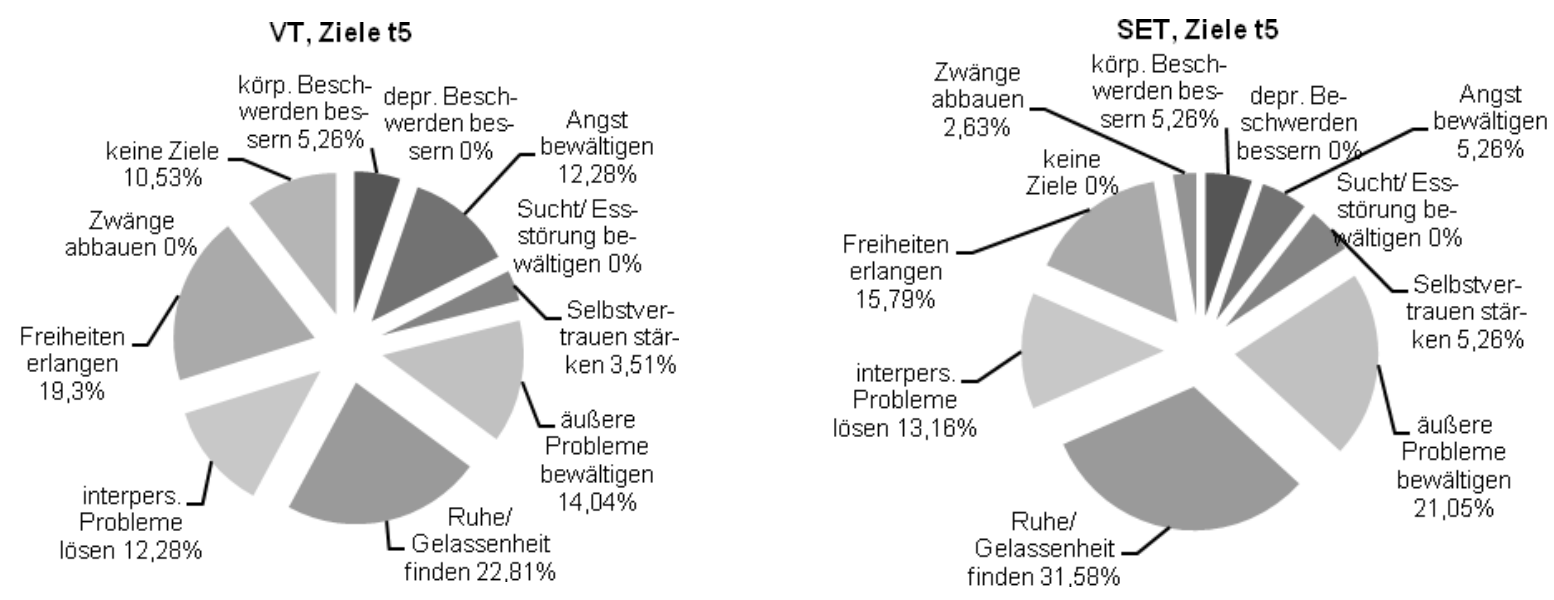

Körperliche Beschwerden und das Ziel, diese zu bessern:

Bei der genauen Analyse von Kategorie „körperliche Beschwerden“ (P1) des Kategoriensystems ist bemerkenswert, dass psychodynamisch behandelte Patienten zu t1 (3. Sitzung) und t2 (12. Sitzung) prozentual häufiger körperliche Beschwerden als Problem schildern. Unter beiden Therapieverfahren kann anfangs (zwischen $\mathrm{t} 1$ und t2) ein Rückgang dieser Angaben erreicht werden, bis zum Ende der Therapie kommt es dann aber in beiden Untersuchungsgruppen zu einem Anstieg von Problemen dieser Kategorie.

Abbildung 15: zeitliche Entwicklung von Kategorie P1 („körperliche Beschwerden“) des Kategoriensystems, Vergleich der Therapiegruppen

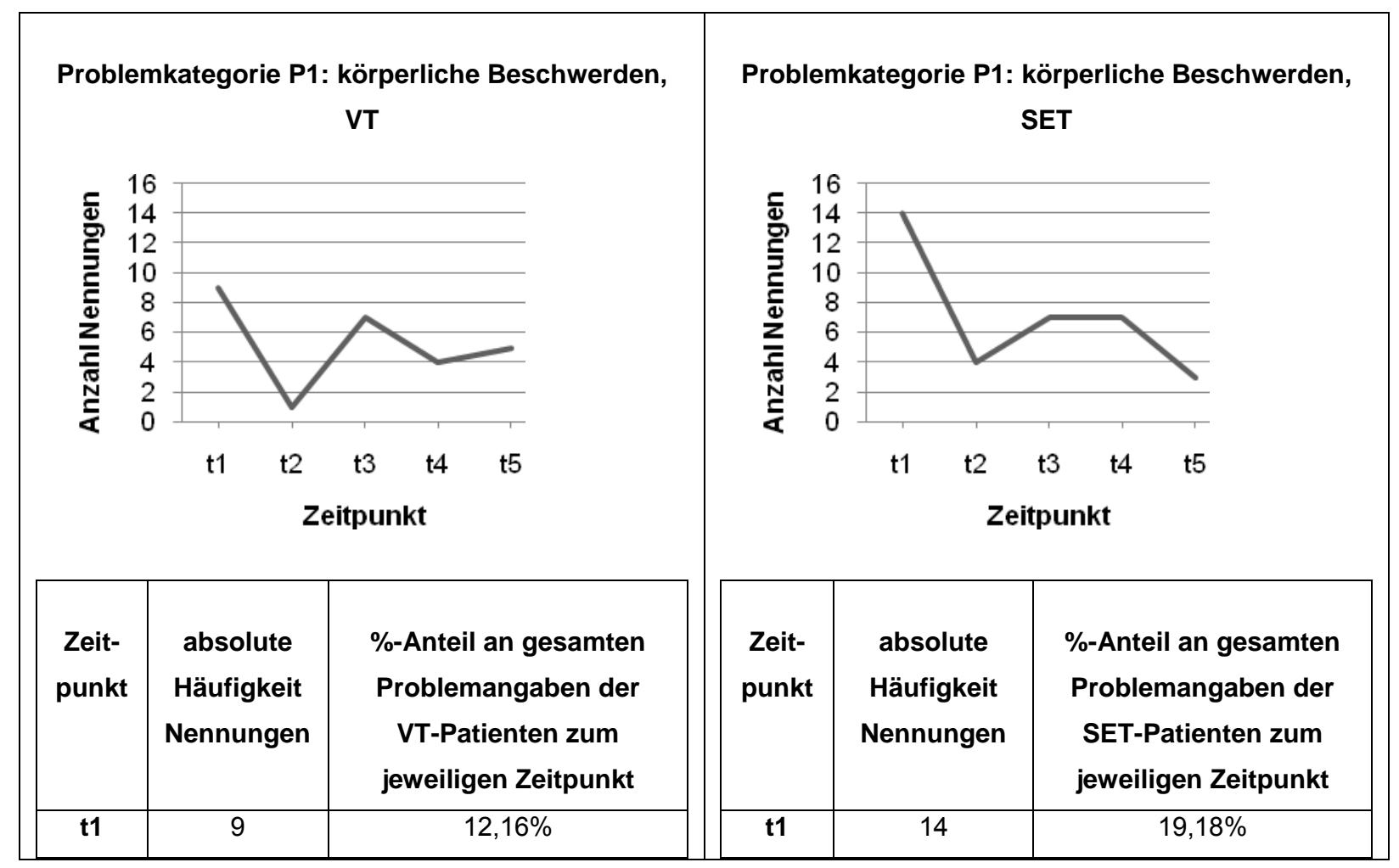




\begin{tabular}{|r|c|c|}
\hline $\mathbf{t 2}$ & 1 & $1,47 \%$ \\
\hline $\mathbf{t 3}$ & 7 & $12,28 \%$ \\
\hline $\mathbf{t 4}$ & 4 & $6,25 \%$ \\
\hline $\mathbf{t 5}$ & 5 & $9,26 \%$ \\
\hline
\end{tabular}

\begin{tabular}{|c|c|c|}
\hline $\mathbf{t 2}$ & 4 & $5,97 \%$ \\
\hline $\mathbf{t 3}$ & 7 & $12,28 \%$ \\
\hline $\mathbf{t 4}$ & 7 & $10,77 \%$ \\
\hline $\mathbf{t 5}$ & 3 & $7,69 \%$ \\
\hline
\end{tabular}

Bezüglich genannter Ziele der Patienten, welche die Besserung körperlicher Beschwerden beinhalten, unterscheiden sich die beiden Therapierichtungen prozentual kaum voneinander. Die Abnahme derartiger Zielinhalte ist von Befragungszeitpunkt t1 (3. Therapiesitzung) zu t2 (12. Therapiesitzung) unter beiden Therapieverfahren am stärksten.

Abbildung 16: zeitliche Entwicklung von Kategorie Z1 („körperliche Beschwerden bessern“) des Kategoriensystems, Vergleich der Therapiegruppen

Anmerkung: die unterschiedlichen Prozentsätze gleicher Zahlen der absoluten Nennungen kommen dadurch zustande, dass der Prozentsatz den Anteil an allen zum jeweiligen Zeitpunkt vorliegenden Zielaussagen einer Gruppe bildet.

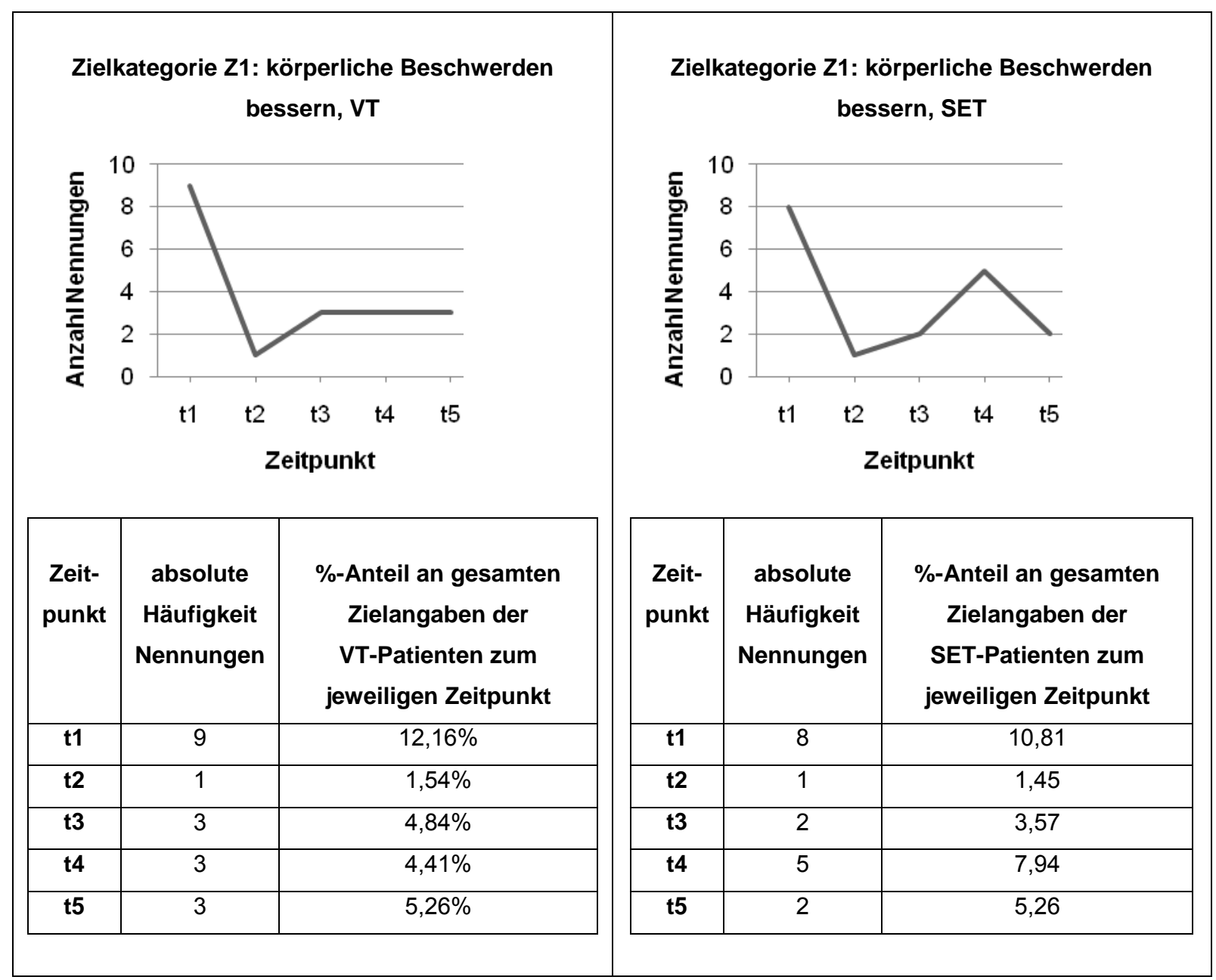


Angst und das Ziel, diese zu bewältigen:

Wie unter 3.4 bereits erläutert, nannten Patienten, welche verhaltenstherapeutisch behandelt wurden, zu Therapiebeginn häufiger Probleme mit Angstinhalten. Der Anteil dieser Kategorie an den zu t1 genannten Problemen einer Therapiegruppe beträgt in der VT-Gruppe 41,9\% und in der Gruppe der SET-Patienten 23,29\%. Der weitere zeitliche Verlauf dieser Kategorie ist dann unter beiden Behandlungsformen ähnlich, Angst wird immer seltener als Problem genannt. Am Ende der Therapie (Befragungszeitpunkt $\mathrm{t} 3$ ) beinhalten nach VT $24,56 \%$ der hier angegebenen Probleme Angst. Nach psychodynamischer Behandlung sind es $14,04 \%$. Auch nach Therapieende sinken die Nennungen von Problemen im Zusammenhang mit Angst prozentual in beiden Therapiegruppen.

Abbildung 17: zeitliche Entwicklung von Kategorie P3 („Angst“) des Kategoriensystems, Vergleich der Therapiegruppen

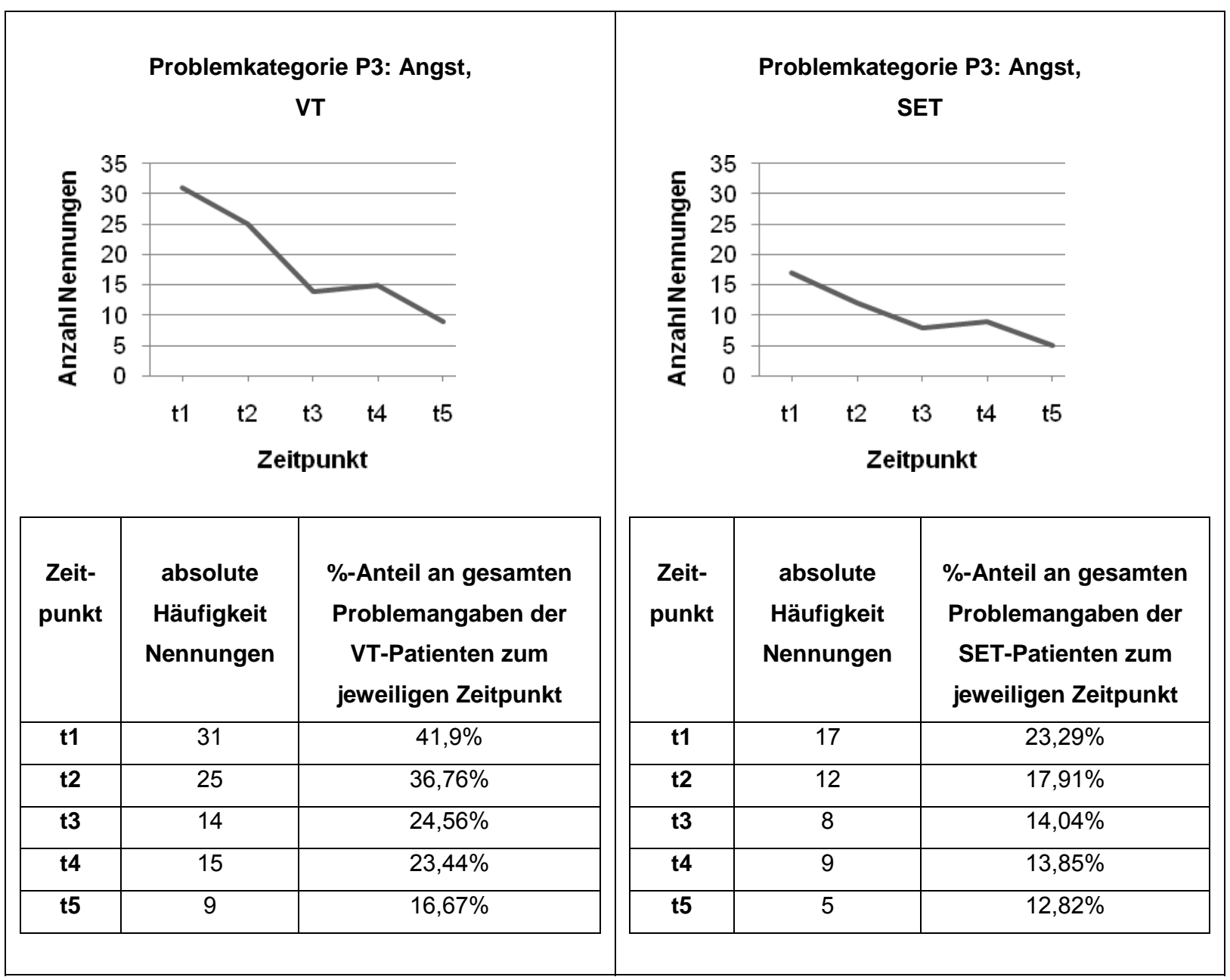


Zielaussagen, Angst bewältigen zu wollen, treten bei verhaltenstherapeutisch behandelten Patienten prozentual nur zu t 1 und t5 häufiger als bei SET-Patienten auf. Von t2 bis t4 ähneln sich hier beide Therapieformen in den Prozentsätzen.

Abbildung 18: zeitliche Entwicklung von Kategorie Z3 („Angst bewältigen“) des Kategoriensystems, Vergleich der Therapiegruppen

\begin{tabular}{|c|c|c|c|c|c|}
\hline \multicolumn{2}{|c|}{ Zielkategorie Z3: Angst bewältigen, } & \multicolumn{3}{|c|}{ Zielkategorie Z3: Angst bewältigen, } \\
\hline
\end{tabular}

Ungelöste Probleme und Konflikte sowie das Ziel, äußere Probleme zu bewältigen:

Probleme der Kategorie „ungelöste Probleme/ Konflikte, z.B. Altag und Beruf“ (P6) werden prozentual im Verlauf der Studie von Patienten der VT zunehmend häufiger genannt. Demnach liegt der Anteil solcher Aussagen an allen angegebenen Problemen der VT-Gruppe zu t1 bei 10,81\%, zu t3 $26,32 \%$ und zu t5 53,7\%.

Unter psychodynamischer Therapie liegen die Prozentsätze dieser Kategorie zu t1 bei $27,4 \%$, zu t3 35,09\%, und zu t5 43,59\%. Insgesamt bleibt unter SET die absolute Häufigkeit der Nennungen von Problemen dieser Kategorie relativ konstant, es steigt jedoch mit der Zeit der prozentuale Anteil dieser Nennungen an den Angaben in der Gruppe, da Patienten zunehmend häufiger keine Angaben zu ihren Problemen mehr 
machten (siehe dazu Informationen unter 3.9).

Abbildung 19: zeitliche Entwicklung von Kategorie P6 („ungelöste Problemel Konflikte, z.B. Alltag und Beruf, Finanzen“) des Kategoriensystems, Vergleich der Therapiegruppen

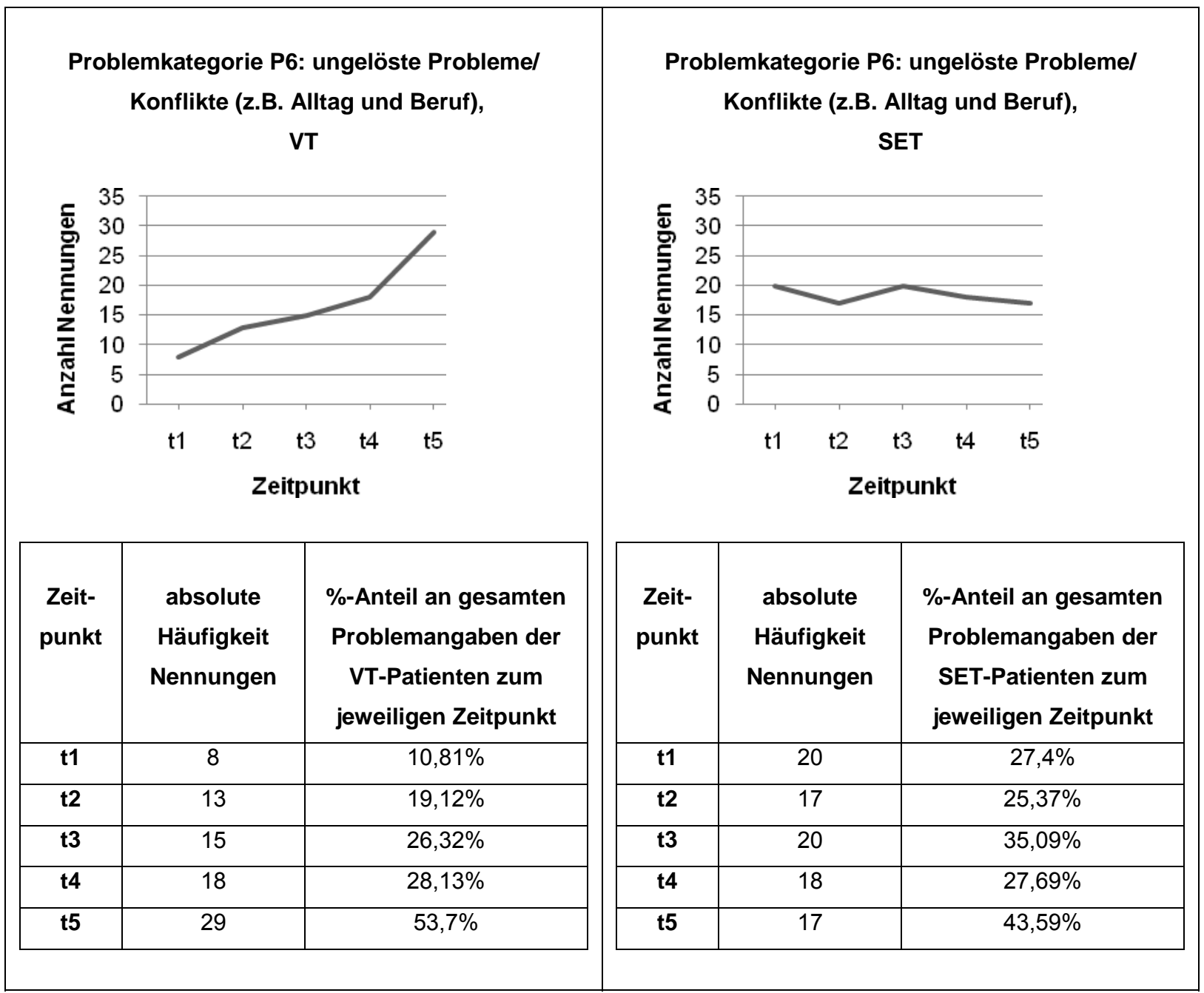

Analog zur zeitlichen Zunahme der Problemangaben im Bereich ungelöster Probleme z.B. des Alltags in der Gruppe der verhaltenstherapeutisch behandelten Patienten, steigt hier in der Formulierung von Zielen der Wunsch, diese („äußeren“) Probleme zu bewältigen (Kategorie Z6). Aber auch die Ziele der psychodynamisch behandelten Studienteilnehmer beinhalten im Verlauf zunehmend Angaben dieser Kategorie, so dass sich beide Therapiegruppen angleichen. 
Abbildung 20: zeitliche Entwicklung von Kategorie Z6 („(äußere) Probleme

bewältigen, z.B. Beruf, Finanzen, Alltag“) des Kategoriensystems, Vergleich der Therapiegruppen

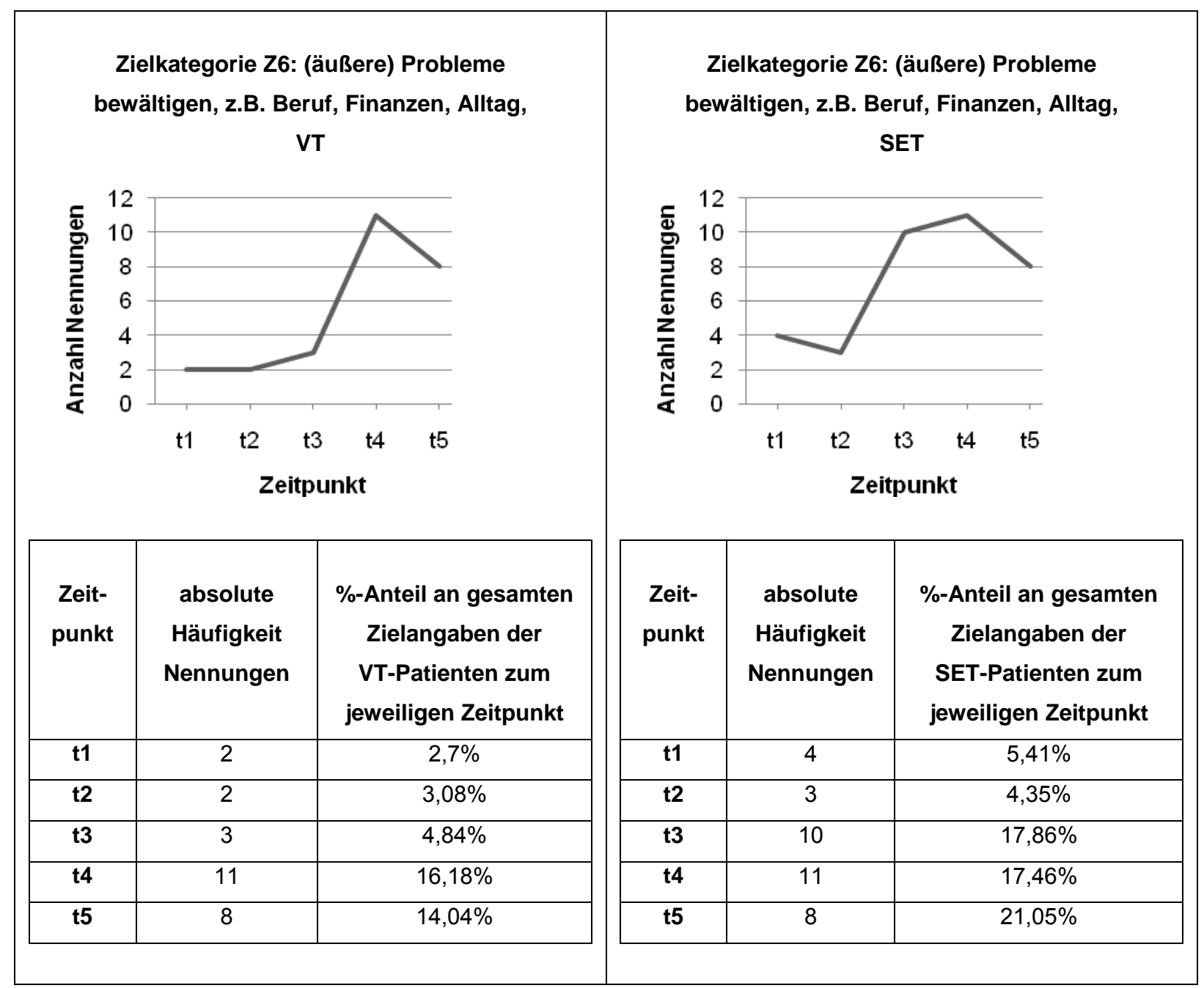

Selbstbezogene Probleme und das Ziel, Ruhe und Gelassenheit zu finden:

Einen bei beiden Behandlungsgruppen in den Anzahlen der Aussagen ähnlichen Verlauf ergibt Kategorie „sonstige selbstbezogene Probleme“ (Kategorie P7).

Abbildung 21: zeitliche Entwicklung von Kategorie P7 („sonstige selbstbezogene Probleme“) des Kategoriensystems, Vergleich der Therapiegruppen

\begin{tabular}{|c|c|}
\hline $\begin{array}{c}\text { Problemkategorie P7: sonstige selbstbezogene } \\
\text { Probleme, VT }\end{array}$ & $\begin{array}{c}\text { Problemkategorie P7: sonstige selbstbezogene } \\
\end{array}$ \\
& \\
\hline
\end{tabular}




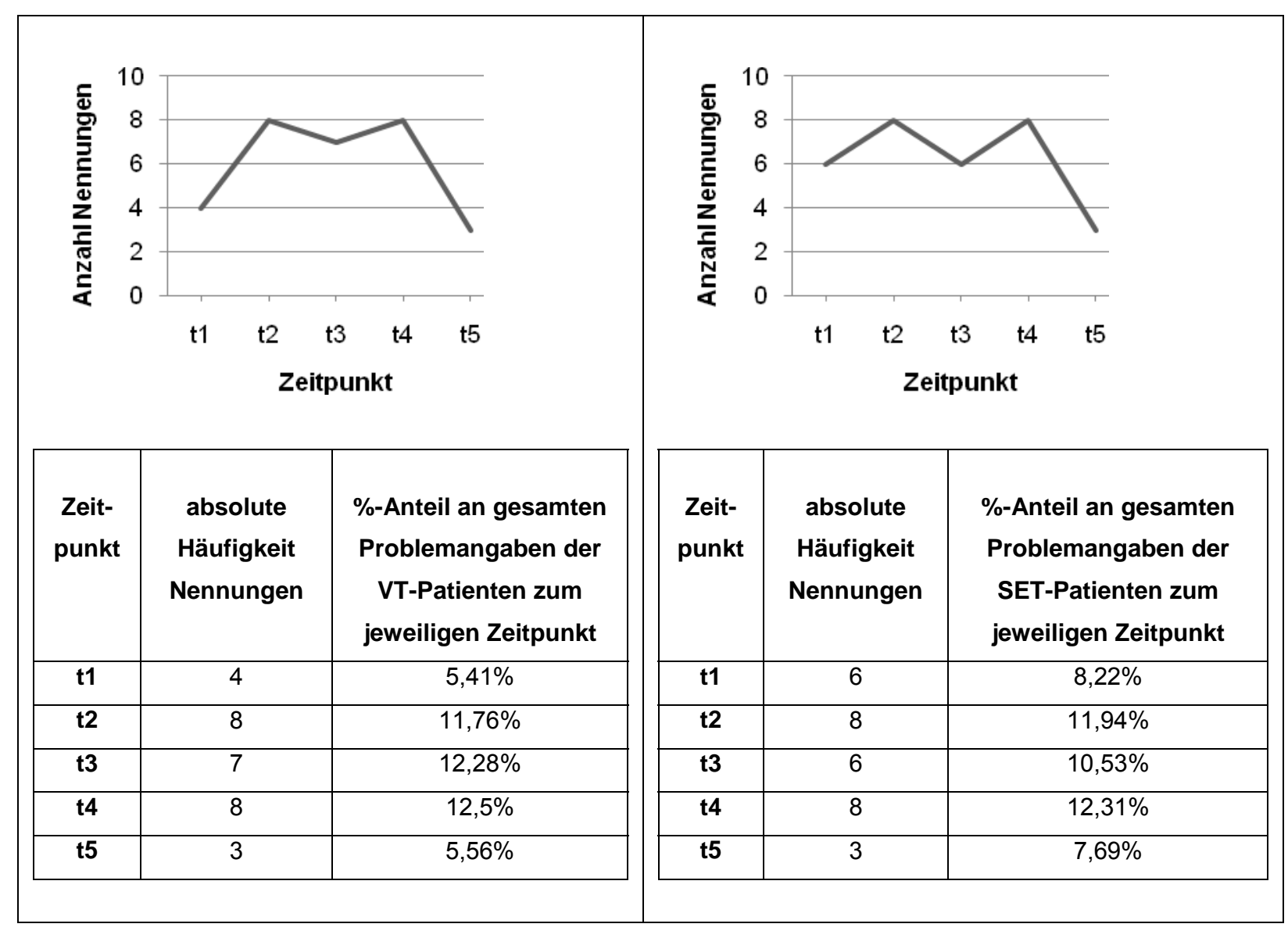

Ziele, welche inhaltlich der Kategorie „Ruhe/ Gelassenheit finden“ (Z7) zugeordnet wurden, formulierten im prozentualen Vergleich beider Therapierichtungen stets häufiger Patienten, die psychodynamisch behandelt wurden.

Abbildung 22: zeitliche Entwicklung von Kategorie Z7 („Ruhe/ Gelassenheit finden“) des Kategoriensystems, Vergleich der Therapiegruppen

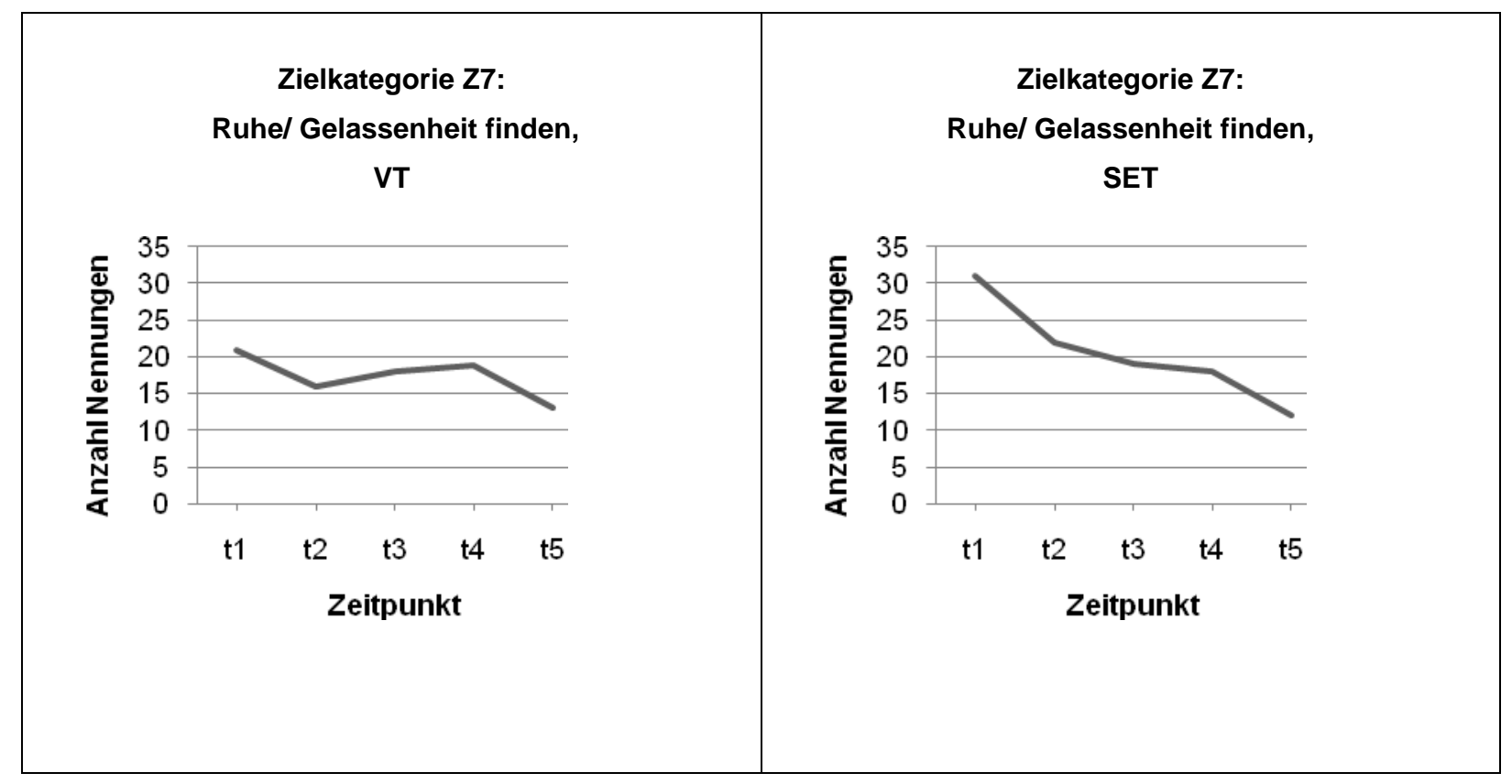




\begin{tabular}{|c|c|c|c|c|c|}
\hline $\begin{array}{l}\text { Zeit- } \\
\text { punkt }\end{array}$ & $\begin{array}{c}\text { absolute } \\
\text { Häufigkeit } \\
\text { Nennungen }\end{array}$ & $\begin{array}{c}\text { \%-Anteil an gesamten } \\
\text { Zielangaben der } \\
\text { VT-Patienten zum } \\
\text { jeweiligen Zeitpunkt }\end{array}$ & $\begin{array}{l}\text { Zeit- } \\
\text { punkt }\end{array}$ & $\begin{array}{c}\text { absolute } \\
\text { Häufigkeit } \\
\text { Nennungen }\end{array}$ & $\begin{array}{l}\text { \%-Anteil angesamten } \\
\text { Zielangaben der } \\
\text { SET-Patienten zum } \\
\text { jeweiligen Zeitpunkt }\end{array}$ \\
\hline t1 & 21 & $28,38 \%$ & t1 & 31 & $41,89 \%$ \\
\hline t2 & 16 & $24,62 \%$ & t2 & 22 & $31,88 \%$ \\
\hline t3 & 18 & $29,03 \%$ & t3 & 19 & $33,93 \%$ \\
\hline t4 & 19 & $27,94 \%$ & t4 & 18 & $28,57 \%$ \\
\hline t5 & 13 & $22,81 \%$ & t5 & 12 & $31,58 \%$ \\
\hline
\end{tabular}

Interpersonelle Probleme und das Ziel, diese zu lösen:

Die angegebenen Probleme und Ziele der Patienten, welche im Kategoriensystem der Kategorie „interpersonelle Probleme“ bzw. „interpersonelle Probleme lösen“ (P8, Z8) entsprechen, wurden verschiedenen Sub-Kategorien (8k, 8d, 8s, 8x) zugeordnet. Um eine bessere Übersicht dieser Kategorie zu erlangen, wird jedoch erst die Gesamtsumme von Kategorie P8 bzw. Z8 nach Therapierichtungen getrennt beurteilt. Interpersonelle Probleme wurden zu Therapiebeginn (t1) häufiger von Patienten der VT-Untersuchungsgruppe angegeben, hier liegt der Anteil an allen genannten Problemen bei 9,46\%, unter SET bei 4,11\%. Während bei VT zu t2 der Prozentsatz relativ gleich bleibt $(10,29 \%)$, steigt diese Kategorie im gleichen Zeitraum bei SET an $(16,42 \%)$.

Auch im weiteren Verlauf bleibt der Prozentsatz bei VT-Patienten fast unverändert, während es bis zum Zeitpunkt t5 (12 Monate nach der Therapie) in der SET-Gruppe zu einer prozentualen Abnahme derartiger Probleme kommt.

Abbildung 23: zeitliche Entwicklung von Kategorie P8 („interpersonelle Probleme“) des Kategoriensystems, Sub-Kategorien zusammengezählt, Vergleich der Therapiegruppen

\begin{tabular}{|c|c|}
\hline $\begin{array}{c}\text { Problemkategorie P8: interpersonelle } \\
\text { Probleme, Sub-Kategorien summiert, }\end{array}$ & $\begin{array}{c}\text { Problemkategorie P8: interpersonelle } \\
\text { PT }\end{array}$ \\
& SET \\
\hline
\end{tabular}




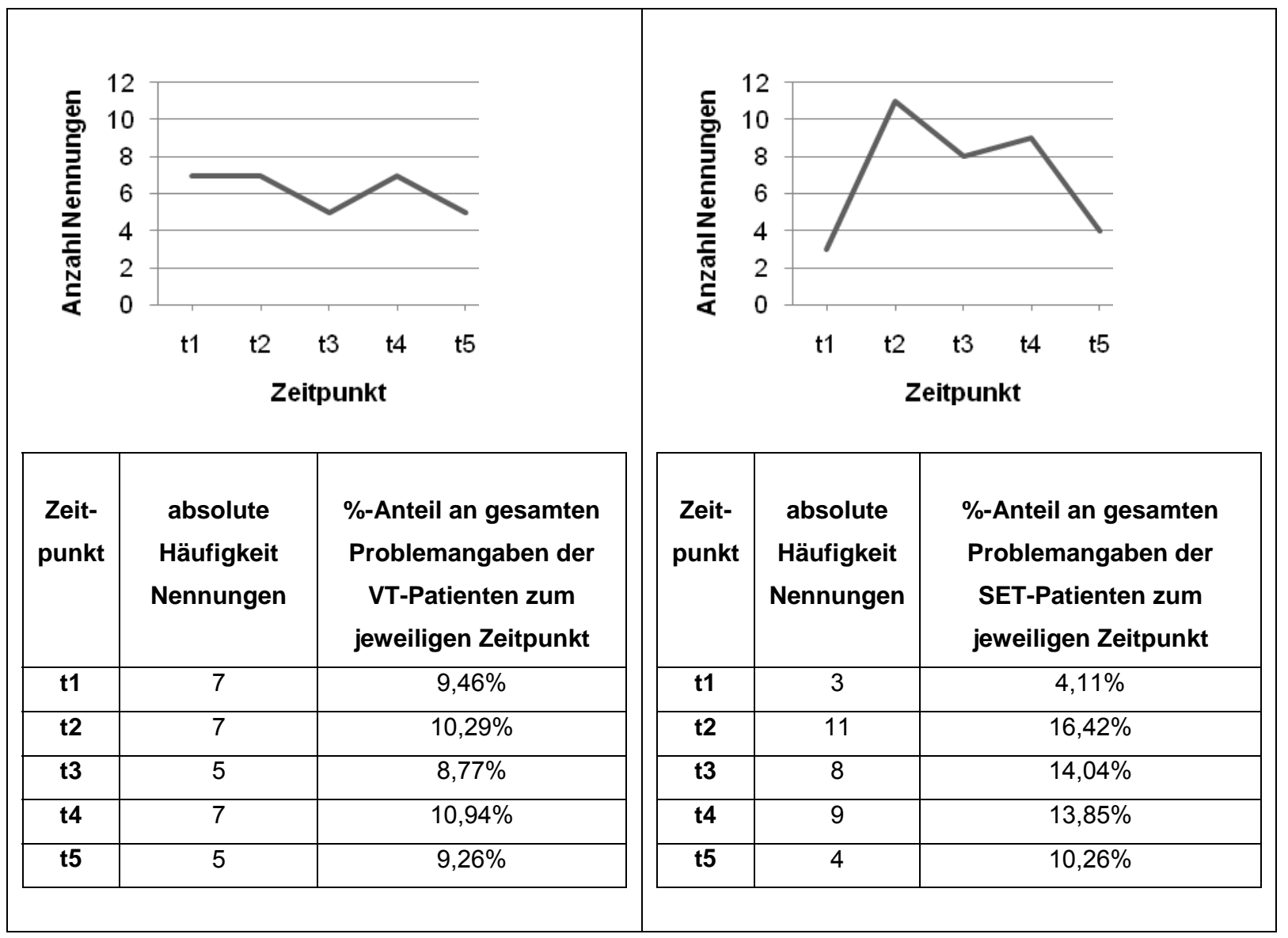

Ziele der Kategorie „interpersonelle Probleme Iösen“ (Z8) werden zu t1 prozentual häufiger von Patienten der VT angegeben, der Prozentsatz an allen zu diesem Zeitpunkt angegebenen Zielen beträgt 9,46\%, während er unter SET bei 2,7\% liegt. Im weiteren Verlauf steigt dieser Prozentsatz in der SET-Gruppe auf 13,16\% zu t5 an. In der VT-Behandlungsgruppe ist der höchste Wert mit 14,71\% zum Zeitpunkt $\mathrm{t} 4 \mathrm{zu}$ verzeichnen.

Abbildung 24: zeitliche Entwicklung von Kategorie Z8 (,interpersonelle Probleme lösen“) des Kategoriensystems, Sub-Kategorien zusammengezählt, Vergleich der Therapiegruppen

Zielkategorie Z8: interpersonelle Probleme lösen, Sub-Kategorien summiert, VT
Zielkategorie Z8: interpersonelle Probleme lösen, Sub-Kategorien summiert,

SET 


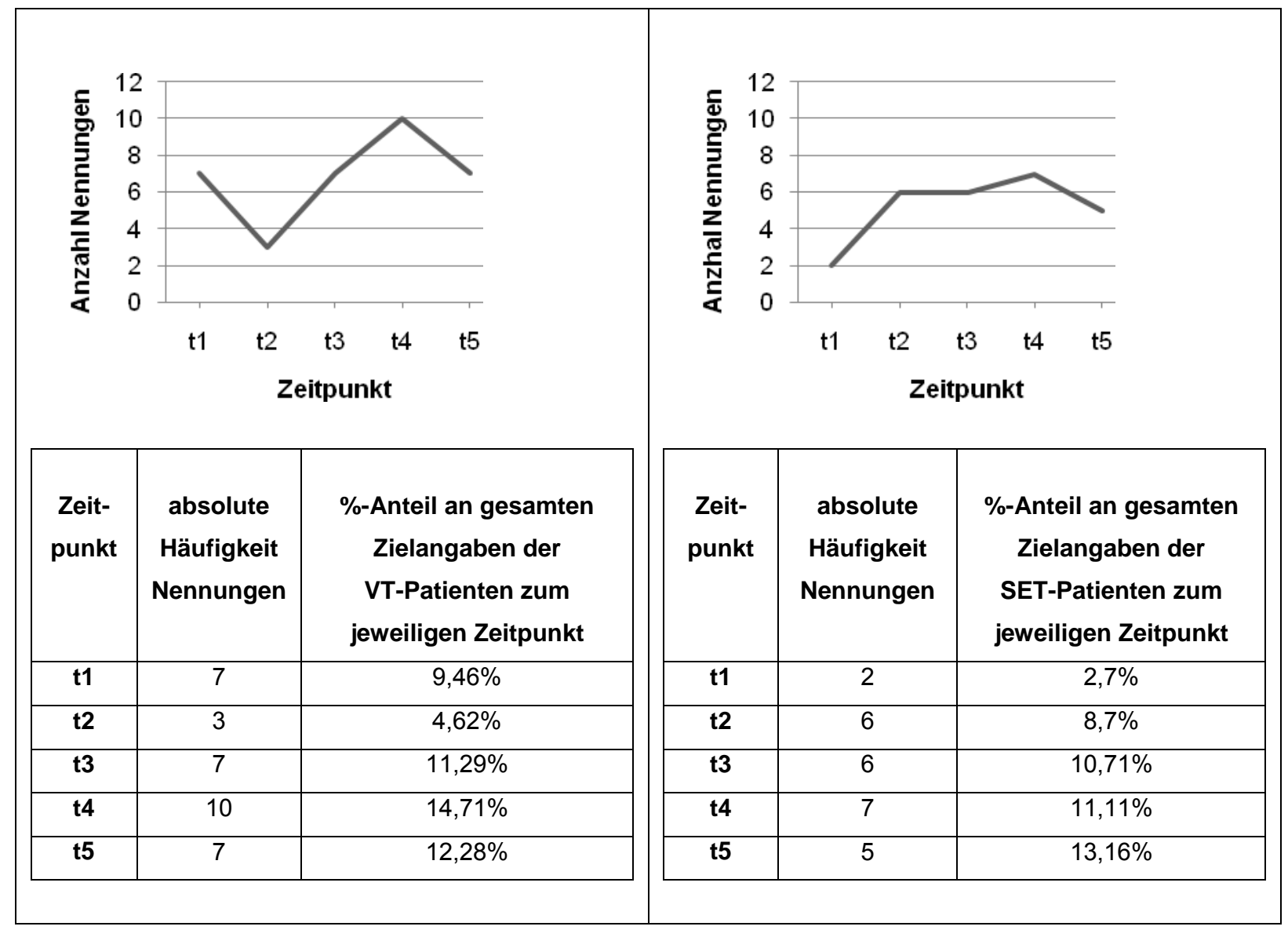

Die Unterkategorien von P8 und Z8 weisen meist nur geringe absolute Häufigkeiten an Problemen und Zielen auf, exemplarisch wird eine Unterkategorie näher erläutert: Kategorie „interpersonelle Probleme: Selbstverlust; Angst vor Ablehnung, Wunsch nach Anerkennung, etc." (Kategorie P8s) wird zu Therapiebeginn (t1) prozentual häufiger von verhaltenstherapeutisch betreuten Personen angegeben. Der höchste prozentuale Anteil dieser Sub-Kategorie an allen genannten Problemen einer Therapiegruppe ist mit 7,41\% bei Patienten unter VT zu t5 und unter SET mit 7,46\% zu t2 zu beobachten.

Tabelle 10: zeitliche Entwicklung von Kategorie P8s (,interpersonelle Probleme: Selbstverlust“) des Kategoriensystems, Vergleich der Therapiegruppen

\begin{tabular}{|c|c|c|c|c|c|}
\hline \multicolumn{6}{|c|}{ Problemkategorie 8s „interpersonelle Probleme: Selbstverlust“ } \\
\hline \multicolumn{3}{|c|}{ VT } & \multicolumn{3}{|c|}{ SET } \\
\hline $\begin{array}{l}\text { Zeit- } \\
\text { punkt }\end{array}$ & $\begin{array}{c}\text { absolute } \\
\text { Häufigkeit } \\
\text { Nennungen }\end{array}$ & $\begin{array}{c}\text { \%-Anteil an gesamten } \\
\text { Problemangaben der } \\
\text { VT-Patienten zum } \\
\text { jeweiligen Zeitpunkt }\end{array}$ & $\begin{array}{l}\text { Zeit- } \\
\text { punkt }\end{array}$ & $\begin{array}{c}\text { absolute } \\
\text { Häufigkeit } \\
\text { Nennungen }\end{array}$ & $\begin{array}{c}\text { \%-Anteil an gesamten } \\
\text { Problemangaben der } \\
\text { SET-Patienten zum } \\
\text { jeweiligen Zeitpunkt }\end{array}$ \\
\hline
\end{tabular}




\begin{tabular}{|c|c|c|c|c|c|}
\hline t1 & 4 & $5,41 \%$ & t1 & 1 & $1,37 \%$ \\
\hline t2 & 4 & $5,88 \%$ & t2 & 5 & $7,46 \%$ \\
\hline t3 & 2 & $3,51 \%$ & t3 & 3 & $5,26 \%$ \\
\hline t4 & 2 & $3,13 \%$ & t4 & 3 & $4,62 \%$ \\
\hline t5 & 4 & $7,41 \%$ & t5 & 1 & $2,56 \%$ \\
\hline
\end{tabular}

\section{Ziel Freiheiten und Fähigkeiten zu erlangen:}

Eine weitere Kategorie, welcher relativ häufig Ziele zugeordnet werden konnten, ist „Freiheiten/ Fähigkeiten erlangen“ (Kategorie Z9). Wird in dieser Zielkategorie nach den beiden Therapieformen differenziert, so zeigt sich, dass verhaltenstherapeutisch behandelte Patienten ab dem Zeitpunkt t2 (12. Therapiesitzung) häufiger Ziele dieser Kategorie angeben. Zum Behandlungsende (Befragungszeitpunkt t3) zeigt sich prozentual der größte Unterschied zwischen beiden Therapierichtungen: Während unter VT $27,42 \%$ der in dieser Gruppe angegebenen Ziele Kategorie Z9 zugeordnet werden konnten, sind es zum gleichen Zeitpunkt unter psychodynamischer Therapie nur $8,93 \%$.

Abbildung 25: zeitliche Entwicklung von Kategorie Z9 („Freiheiten/ Fähigkeiten erlangen“) des Kategoriensystems, Vergleich der Therapiegruppen

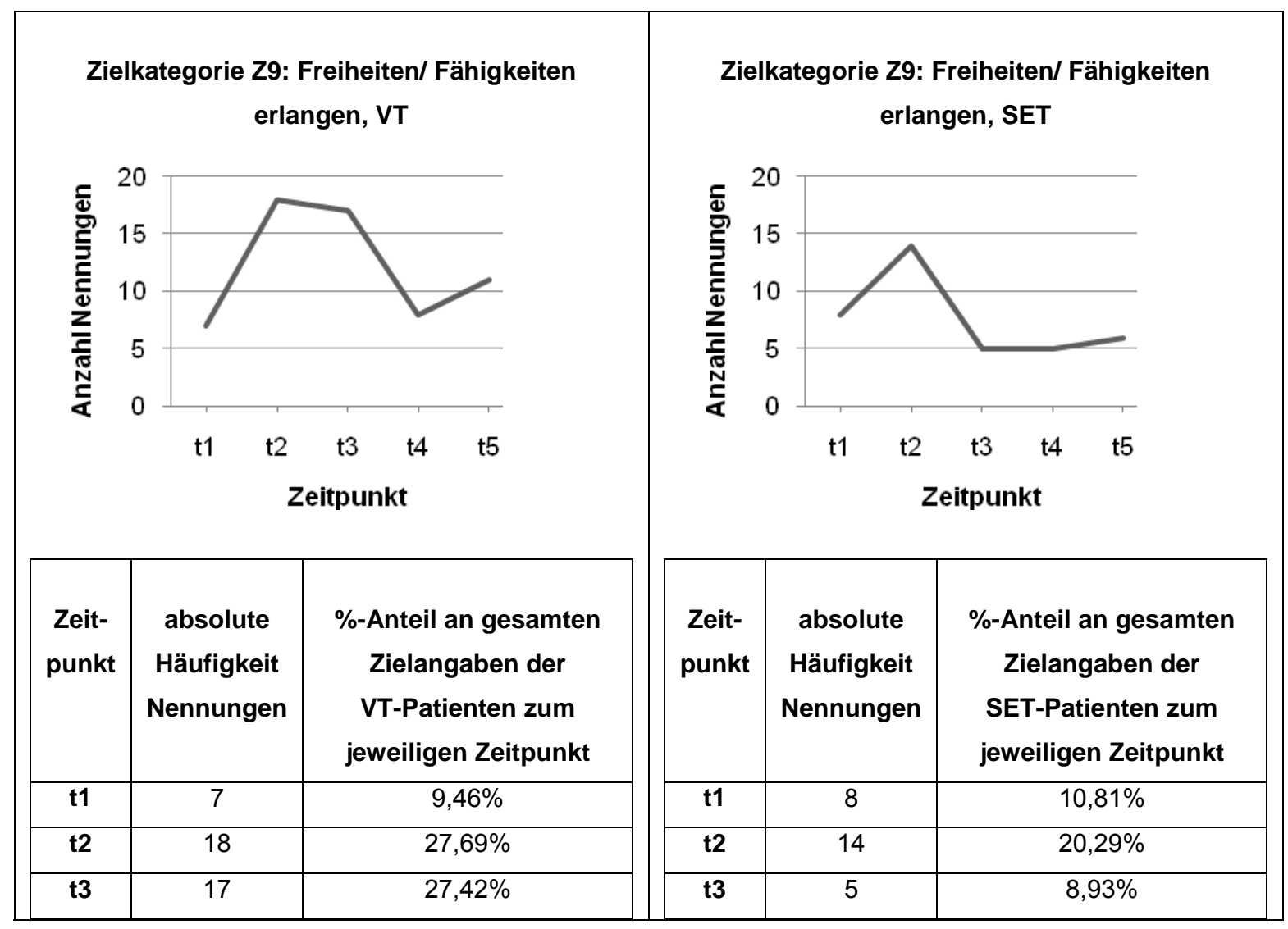




\begin{tabular}{|c|c|c||c|c|c|}
\hline $\mathbf{t} 4$ & 8 & $11,76 \%$ \\
\hline $\mathbf{t 5}$ & 11 & $19,3 \%$ & $\mathbf{t 4}$ & 5 & $7,94 \%$ \\
\hline $\mathbf{t 5}$ & 6 & $15,79 \%$ \\
\hline
\end{tabular}

Fazit:

Die Resultate der Überprüfung von Hypothese H1.3 wurden im Rahmen dieser Arbeit nicht auf ihre Signifikanz hin untersucht. Deskriptiv weist die nach beiden Therapieformen getrennte Analyse von Problem- und Zielangaben jedoch darauf hin, dass es sowohl Ähnlichkeiten, als auch Unterschiede zwischen den Therapiegruppen hinsichtlich einzelner Inhaltskategorien gibt. Dabei zeigen sich zu Therapiebeginn häufig Unterschiede in den Angaben beider Vergleichsgruppen, welche sich gegen Ende der Behandlung bzw. im KatamneseZeitraum angleichen.

\subsection{Prüfung von Hypothese H1.4}

„Im Therapieverlauf sind systematische Veränderungen festzustellen.“

Mit Überprüfung dieser Hypothese wird untersucht, ob es im Laufe einer psychotherapeutischen Behandlung zu systematischen Veränderungen kommt. Dabei wird nicht nur der zeitliche Rahmen der eigentlichen Therapie (also $\mathrm{t} 1$ bis $\mathrm{t} 3$ ) untersucht, sondern auch die Katamnese-Zeitpunkte t4 (ein halbes Jahr nach Therapieende) und t5 (ein Jahr nach Therapieende) in die Analyse mit einbezogen, da sich Effekte einer Behandlung möglichst auch nach deren Beendigung bemerkbar machen sollten.

Begriffsklärung systematischer Veränderungen:

Unter systematischen Veränderungen wird verstanden, dass im Verlauf einer Psychotherapie sowohl eine inhaltliche Umverteilung, als auch gleichzeitig eine veränderte Wahrnehmung und Bewältigung sowie Bewertung der von Patienten genannten Problemen und Ziele seitens der Patienten selbst stattfindet.

Mittels des Kategoriensystems sind solche Aspekte gut zu überprüfen, es kann beispielsweise zur Symptom-Reduktion von Angst kommen, während Alltagsprobleme zunehmend häufiger genannt werden. 
Mit der Mastery-Awareness-Scale lassen sich systematische Neuerungen in der Wahrnehmung und im Umgang mit Problemen darstellen.

Schließlich können systematische Veränderungen auch unter Zuhilfenahme der Selbsteinschätzung der Patienten nachvollzogen werden. Es kann ferner geprüft werden, ob sich der Leidensdruck unter zu Erhebungszeitpunkt t1 (3. Therapiesitzung) angegebenen Problemen verändert und ob die zu t1 formulierten Ziele im Laufe der Studie (nach Einschätzung der Patienten selbst) erreicht wurden.

Die Prüfung dieser Hypothese stellt mögliche Umverteilungen der Kategorien aller zum jeweiligen Zeitpunkt abgegebenen Problem- und Zielangaben dar, ohne dabei auf einzelne Patienten und deren individuelle Veränderung näher einzugehen.

\subsection{1 Überprüfung mit dem Kategoriensystem}

Inhaltliche Veränderungen der Patienaussagen wurden bereits im Rahmen vorheriger Hypothesenprüfungen untersucht. Diese können hinsichtlich der Analyse der vorliegenden Hypothese als Teilaspekt systematischer Veränderungen verstanden werden.

\subsection{2 Überprüfung mit der Mastery-Awareness-Scale}

Im Folgenden wird die Problemwahrnehmung und -bewältigung der 51 an der Untersuchung teilnehmenden Patienten auf potentiell systematische Veränderungen im Therapieverlauf überprüft. Es sei an dieser Stelle erneut darauf hingewiesen, dass mit der Mastery-Awareness-Scale die Problemangaben, nicht jedoch die Zielangaben der Patienten ausgwertet werden können.

Zusätzliche Abbildungen zu den folgenden Ergebnissen befinden sich aufgrund ihres Umfangs im Anhang.

\section{Problemwahrnehmung aller Patienten:}

Zur Übersicht der Entwicklung der Problemwahrnehmung dienen nachstehende Kreisdiagramme der Zeitpunkte t1 (3. Therapiesitzung), t3 (Behandlungsende) und t5 (ein Jahr nach Behandlungsende). Dabei beziehen sich die Prozentssätze auf 100\% als alle zum jeweiligen Zeitpunkt gemachte Problemaussagen. 
Abbildung 26: Verteilung zu t1, t3 und t5 angegebener Probleme auf Wahrnehmungskategorien der Mastery-Awareness-Scale, alle Patienten
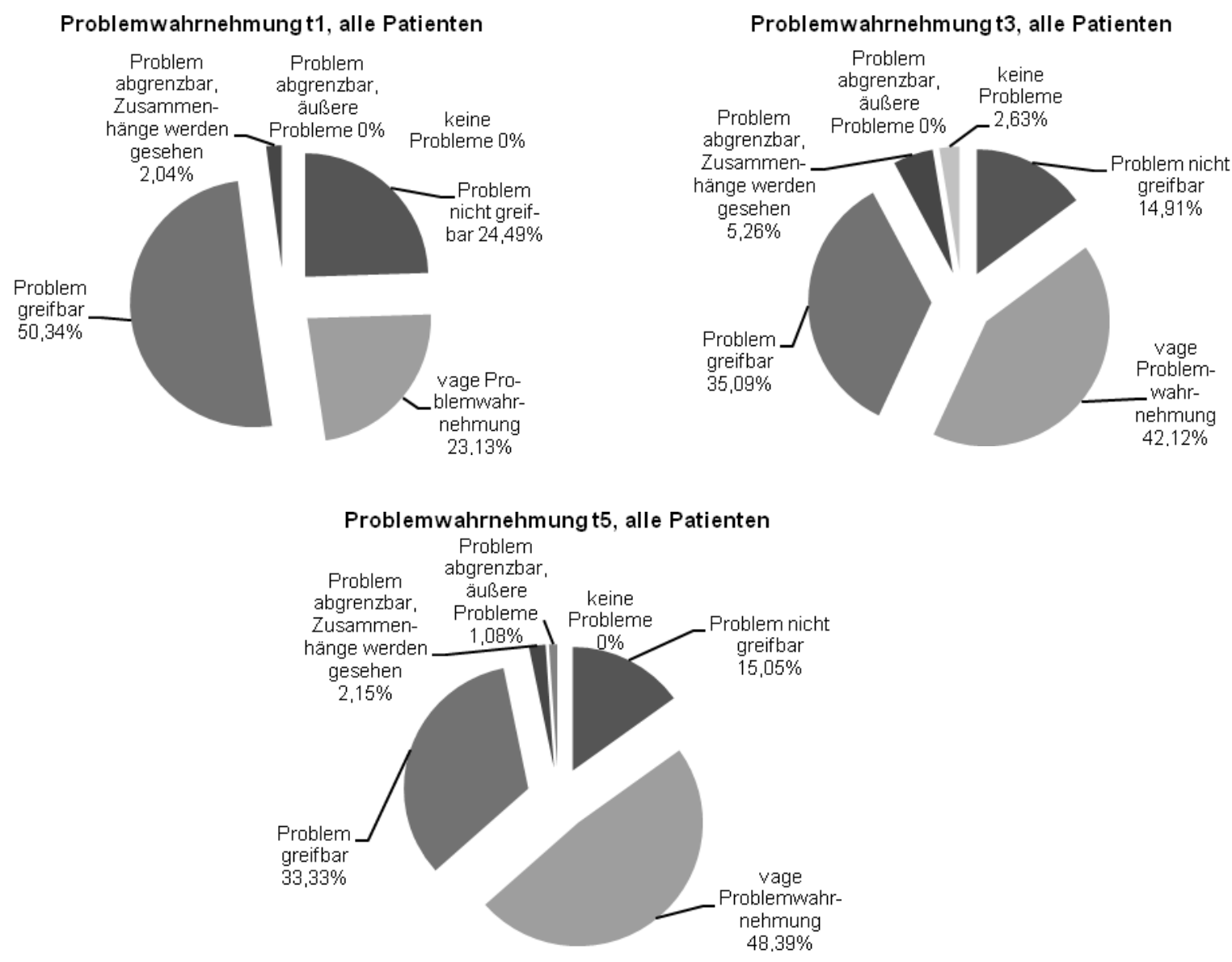

Bezüglich der Problemwahrnehmung werden anfangs häufig nicht greifbare Probleme (Kategorie W1) beschrieben, der Prozentsatz dieser Kategorie an allen zu t1 genannten Problemen liegt bei 24,49\%. Dieser Anteil kann im Verlauf der Therapie auf $14,91 \%$ aller zu Therapieende ( $\mathrm{t} 3$ ) angegebenen Probleme reduziert werden.

Gegesätzlich steigt mit der Zeit der Häufigkeit der Kategorie „vage Problemwahrnehmung" (W2). Der Prozentsatz an allen Problemaussagen liegt zu t1 bei 23,13\% und zu $\mathrm{t} 3$ bei $42,12 \%$. Sogar nach der Therapie, also von $\mathrm{t} 3$ bis $\mathrm{t} 5$, steigt der Anteil vage wahrgenommener Probleme kontinuierlich an (t5 48,39\%).

Als greifbar (Kategorie W3) werden Probleme am häufigsten zu Therapiebeginn (t1) beschrieben. Hier macht diese Kategorie 50,34\% aller angegebenen Probleme aus. $\mathrm{Zu}$ Therapieende (t3) liegt dieser Wert dann nur noch bei 35,09\%, zu t5 (ein Jahr nach Therapieende) bei 33,33\%.

Probleme werden insgesamt sehr selten als abgrenzbar wahrgenommen (Kategorie 4a, 4b), allerdings nimmt die Anzahl in Laufe der Zeit zu. 
Problembewältigung aller Patienten:

Zur Übersicht der Verteilung aller Problemangaben der Zeitpunkte t1, t 3 und t5 auf Bewältigungskategorien der Mastery-Awareness-Scale dienen die folgenden Kreisdiagramme. $100 \%$ entsprechen dabei, wie zuvor, allen zum betreffenden Zeitpunkt gemachten Problemaussagen.

Abbildung 27: Verteilung zu t1, t3 und t5 angegebener Probleme auf Bewältigungskategorien der Mastery-Awareness-Scale, alle Patienten
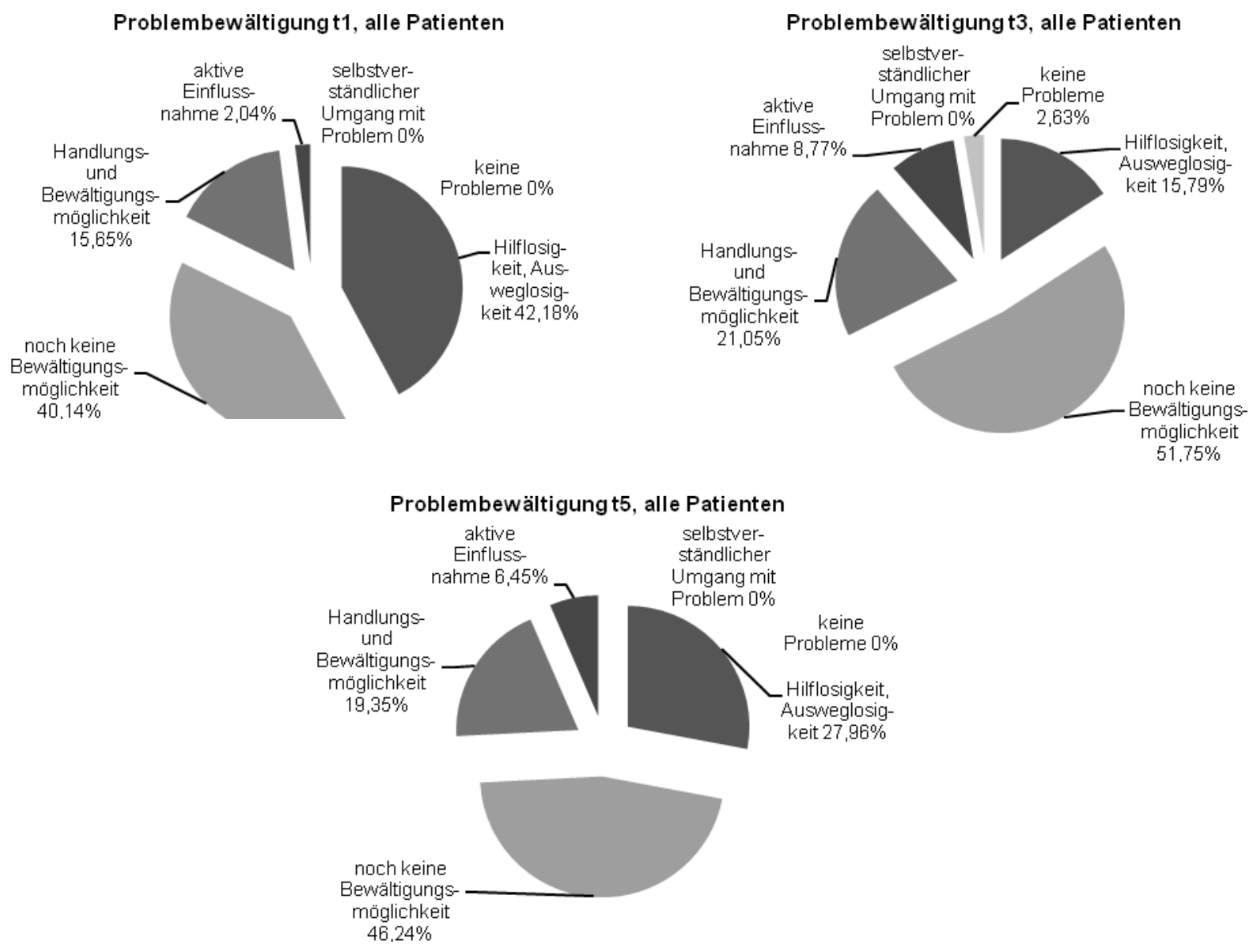

Bezüglich der Bewältigung angegebener Probleme offenbart sich insgesamt im Laufe der Behandlungen ein Rückgang von „Hilflosigkeit, Ausweglosigkeit“ (Kategorie M1) im Umgang mit diesen. Demnach sinkt der prozentuale Anteil an allen genannten Problemen von zu t1 42,18\% auf 15,79\% zum Ende der Therapie (t3). Nach der Behandlung steigt diese Kategorie wieder auf $27,96 \%$ zu Befragungszeitpunkt t5 (ein Jahr nach Therapieende) an.

„Noch keine Bewältigungsmöglichkeit“ genannter Probleme (Kategorie M2, eine Bewältigungsstufe höher als „Hilflosigkeit“) steigt im Verlauf der Therapie von 40,14\% 
aller angegebenen Probleme zu t1 auf 51,75\% zu Therapieende (Zeitpunkt t3). Nach der Behandlung kommt es zu einer Senkung auf 46,24\% zu t5.

„Handlungs- und Bewältigungsmöglichkeiten“ im Umgang mit den genannten Problemen (Kategorie M3) nehmen im Verlauf der Therapie stetig zu. So können zu t1 15,65\% aller Prolemangaben dieser Kategorie zugeordnet werden, während es zu t3 bereits 21,05\%, zu t4 24,03\% und zu t5 19,35\% sind.

Probleme werden im Laufe der Therapie zwar insgesamt selten, aber prozentual und in den absoluten Häufigkeiten zunehmend als abgrenzbar beschrieben. Aktive Einflussnahme (Kategorie M4a) steigt von 2,04\% aller genannten Probleme zu Befragungszeitpunkt t1 auf 8,77\% zu Behandlungsende (t3).

Vergleich beider Therapiegruppen bezüglich ihrer Problemwahrnehmung:

Um die Ergebnisse differenzierter einordnen zu können, wurden diese zusätzlich nach beiden Therapieformen getrennt ausgewertet. Kreisdiagramme der Befragungszeitpunkte t1 (3. Therapiesitzung), t3 (Therapieende) und t5 (ein Jahr nach Therapieende) zeigen die Verteilung der in den beiden Therapiegruppen genannten Probleme auf Wahrnehmungskategorien der Mastery-Awareness-Scale. $100 \%$ entsprechen dabei allen zum jeweiligen Zeitpunkt angebenenen Problemen einer Therapiegruppe.

Abbildung 28: Verteilung zu t1, t3 und t5 angegebener Probleme auf Wahrnehmungskategorien der Mastery-Awareness-Scale, Vergleich der Therapiegruppen
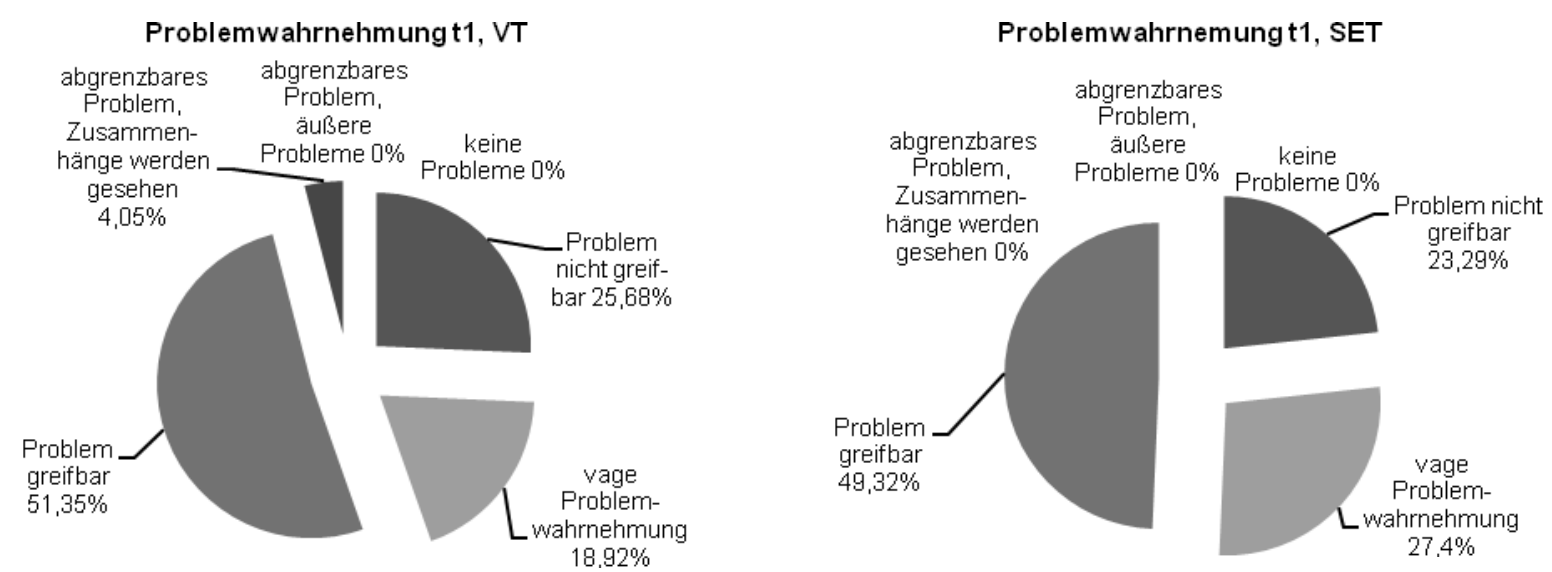


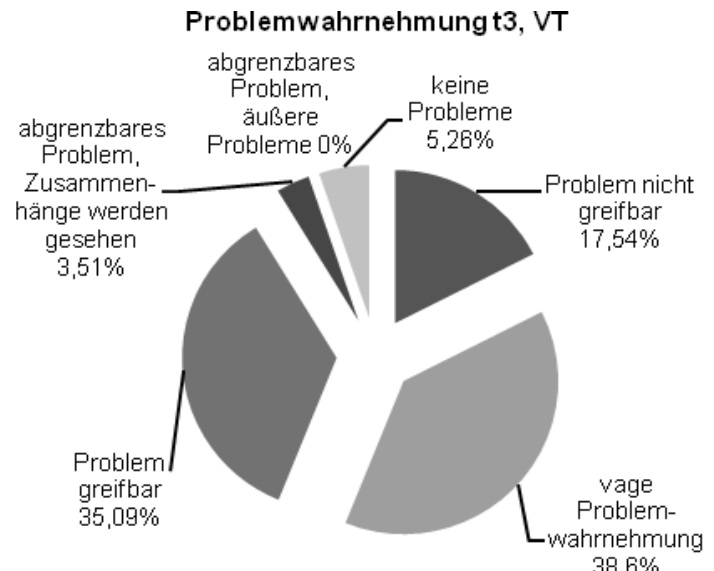

$38.6 \%$

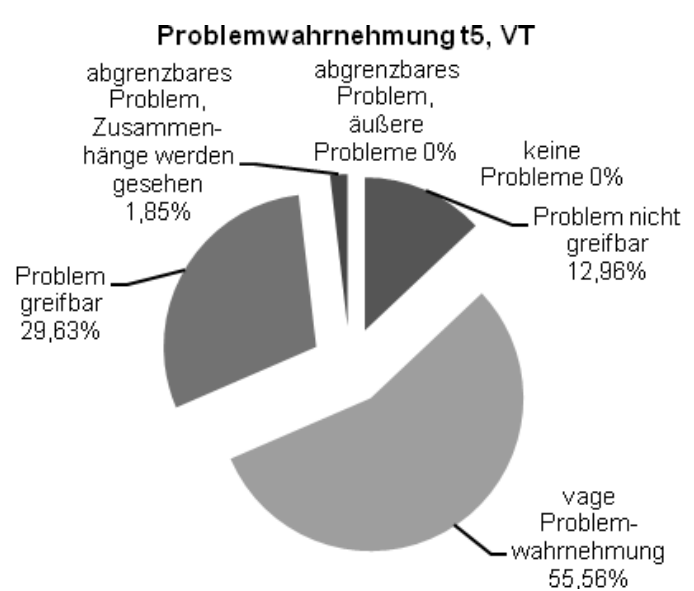

Problemwahrnehmungt3, SET
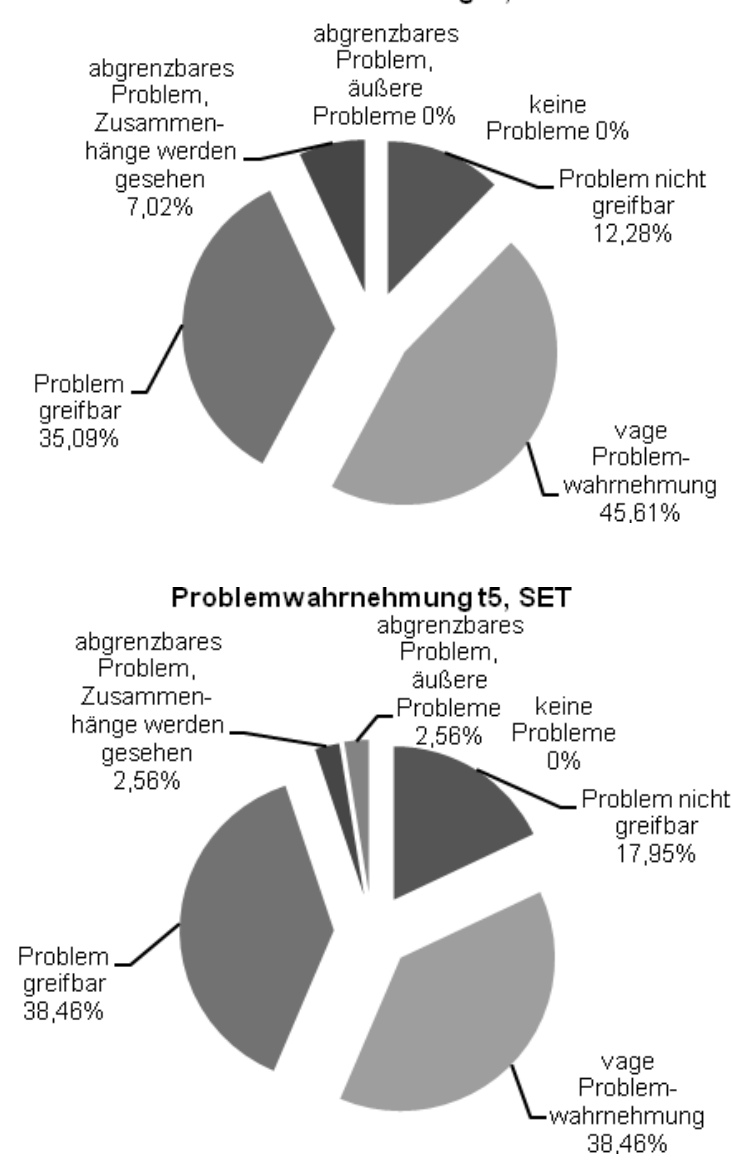

Diese Analyse zeigt, dass unter beiden Psychotherapieverfahren der Anteil nicht greifbarer Probleme (Kategorie W1) im Verlauf der Behandlung sinkt. Demnach liegt dieser an allen zum jeweiligen Zeitpunkt in einer Therapiegruppe genannten Probleme unter VT zu t1 bei $25,68 \%$, zu $\mathrm{t} 3$ bei $17,54 \%$ und zu t5 bei $12,96 \%$. Während der psychodynamischen Psychotherapie beträgt dieser Prozentsatz zu t1 $23,29 \%$, zu t3 $12,28 \%$ und zu t5 17,95\%.

Die vage Wahrnehmung von genannten Problemen (Kategorie W2) steigt hingegen unter beiden Therapieformen an. Der Anteil dieser Kategorie an allen Problemnennungen der Therapiegruppe zum jeweiligen Zeitpunkt liegt zu t1 (3. Therapiesitzung) unter VT bei $18,92 \%$ und unter SET bei $27,4 \%$. Zu Therapieende (t3) liegt dieser Prozentsatz nach VT bei $38,6 \%$ und nach SET bei $45,61 \%$. Bei SETPatienten kommt es nach t4 (ein halbes Jahr nach Therapieende) zu einem deutlichen Rückgang dieser Kategorie von $50,77 \%$ zu t4 auf $38,46 \%$ aller genannten Probleme ein Jahr nach Therapieende (t5). Bei Patienten der VT kommt es hingegen zu einem Anstieg dieser Kategorie von 40,63\% zu t4 auf 55,56\% zu t5.

Probleme, welche als greifbar wahrgenommen wurden (Kategorie W3), sinken 
anteilig im Verlauf beider Therapien. Bemerkenswert ist jedoch der Unterschied der zu t2 genannten Probleme beider Therapiegruppen: Hier beträgt der Anteil dieser Kategorie an allen angegebenen Problemen einer Therapiegruppe unter VT 36,76\% und unter SET 50,75\%. Zu t3 ist der Prozentsatz nach beiden Psychotherapierichtungen gleich hoch und liegt bei 35,09\%. Ein Jahr nach Therapeende (t5) sinkt er nach VT auf 29,63\% und steigt nach SET 38,46\%.

Als abgrenzbar (Kategorie W4a und b) werden in beiden Gruppen nur selten Probleme beschrieben. Kategorie „abgrenzabres Prolem: Zusammenhänge werden gesehen“ (W4a) steigt dabei im Verlauf der Behandlung prozentual unter SET auf 7,02\% der angegebenen Probleme zu t3 (Therapieende), während diese Kategorie im gleichen Zeitraum unter VT weitgehend unverändert bleibt $(4,05 \%$ zu t1, 3,51\% zu t3).

Vergleich beider Therapiegruppen bezüglich ihrer Problembewältigung:

Die in den Therapiegruppen zu Befragungszeitpunkt t1, t3 und t5 genannten Probleme und deren Verteilung auf Bewältigungskategorien der Mastery-AwarenessScale werden mittels einiger Kreisdiagramme verdeutlicht. Alle in einer Therapiegruppe zum jeweiligen Zeitpunkt genannten Probleme entsprechen dabei $100 \%$.

Abbildung 29: Verteilung zu t1, t3 und t5 angegebener Probleme auf Bewältigungskategorien der Mastery-Awareness-Scale, Vergleich der Therapiegruppen
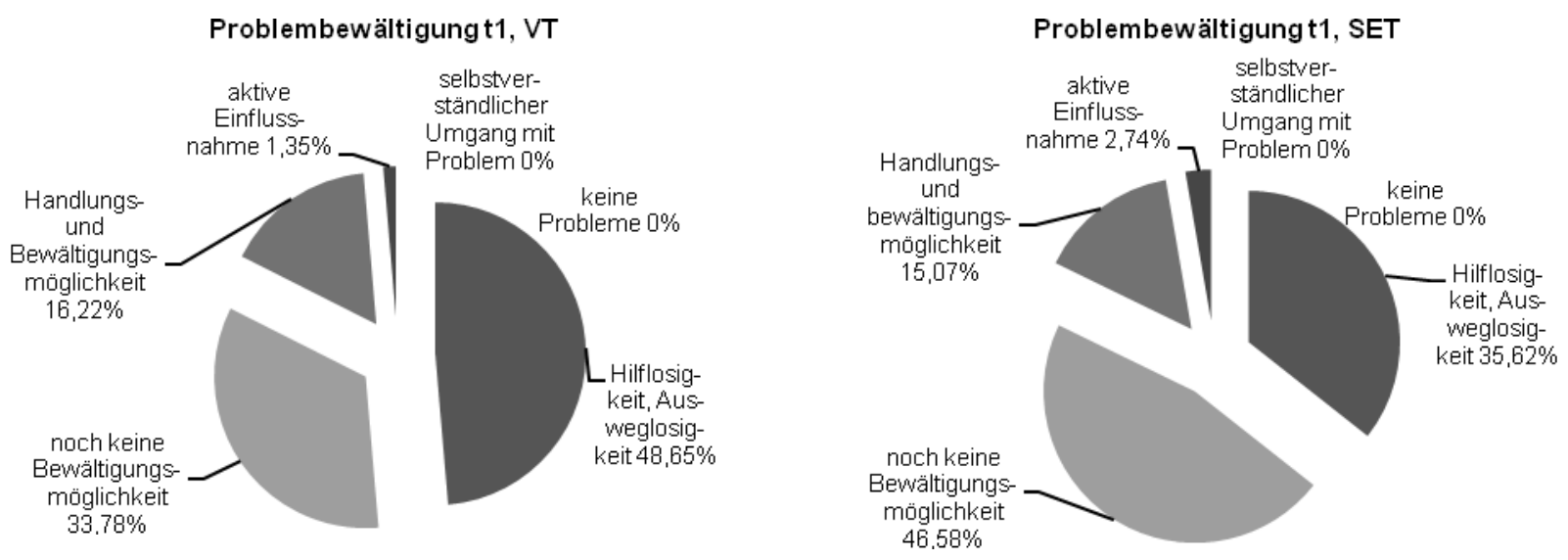

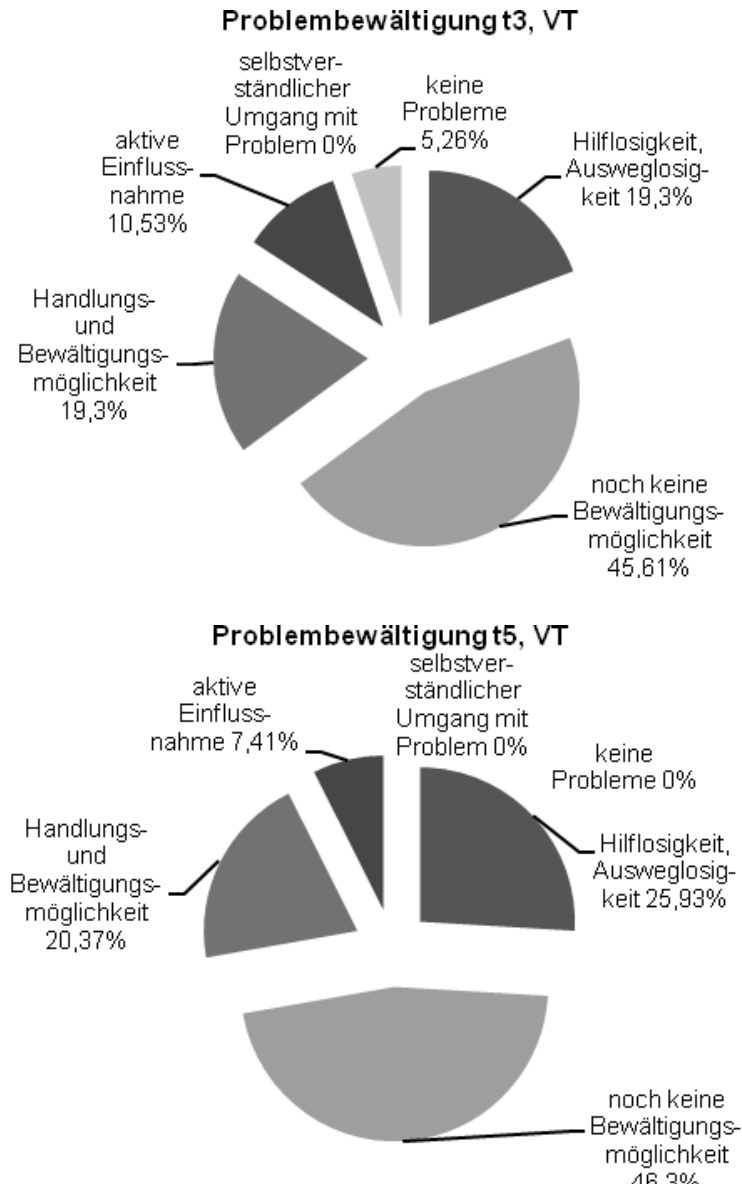
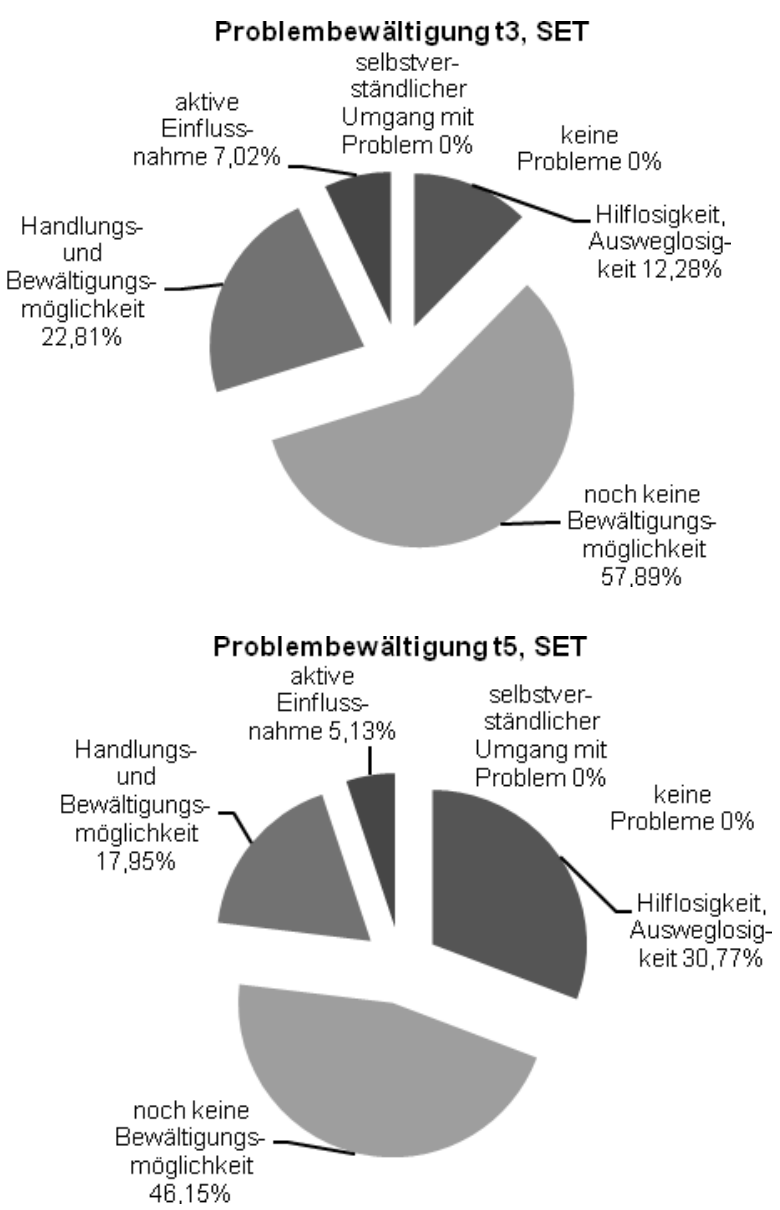

In Bezug auf die Bewältigung von angegebenen Problemen kommt es unter beiden Therapien zu einer Abnahme von Hilflosigkeit (Kategorie M1). Demnach beträgt der Anteil von Problemen, denen sich Patienten hilflos gegenüber sehen, zu Therapiebeginn (t1) in der VT-Gruppe $48,65 \%$, in der SET-Gruppe $35,62 \%$ der angegebenen Probleme. Zu Therapieende (Befragungszeitpunkt $\mathrm{t} 3$ ) beträgt dieser Prozentsatz in der VT-Gruppe nur noch $19,3 \%$ und in der SET-Gruppe nur noch $12,28 \%$ der Problemangaben. Von Befragungszeitpunkt t3 zu t5 (ein Jahr nach Therapieende) kommt es jedoch unter beiden Therapieformen zu einem prozentualen Anstieg der Hilflosigkeit im Umgang mit Problemen, die Prozentsätze betragen zu t5 in der VT-Gruppe $25,93 \%$ und in der SET-Gruppe 30,77\% der Problemangaben.

„Noch keine Bewältigungsmöglichkeit“ (Kategorie M2) bezüglich genannter Probleme steigt prozentual unter beiden Therapieformen im Behandlungsverlauf an, die Anteile an allen Problemangaben einer Therapiegruppe betragen zu t1 unter VT 33,78\%, unter SET 46,58\% und zu Behandlungsende (t3) unter VT 45,61\% sowie unter SETBehandlung 57,89\%. Zur Katamnese-Befragung ein Jahr nach Therapieende (t5) 
beträgt der Prozentsatz dieser Kategorie in beiden Untersuchungsgruppen fast identische Werte, nämlich in der VT-Gruppe 46,3\% und in der psychodynamisch behandelten Patientengruppe (SET) 46,15\% der angegebenen Probleme.

„Handlungs- und Bewältigungsmöglichkeiten“ bezüglich genannter Probleme (Kategorie M3) nehmen im Verlauf der Behandlung in beiden Untersuchungsgruppen prozentual zu. Von zu t1 (3. Therapesitzung) 16,22\% der angeführten Probleme in der VT-Gruppe steigt dieser Wert auf maximal 20,37\% zu t5. In der SET-Gruppe beträgt dieser Prozentsatz zu t1 15,07\% und das Maximum zu t4 32,31\%.

Wie bereits erwähnt, werden Probleme von den Studienteilnehmern insgesamt selten als „abgrenzbar“ mit „aktiver Einflussnahme“ empfunden (Kategorie M4a). Im Verlauf beider Behandlungen werden sie am häufigsten zu Therapeende (t3) derartig wahrgenommen (VT 10,53\%, SET 7,02\% aller Problemangaben zu t3).

\subsubsection{Prüfung der Hypothese mittels Selbsteinschätzung der Patienten bezüg-} lich ihres Leidensdrucks unter zu Befragungszeitpunkt $\mathrm{t} 1$ angegebenen Problemen und der Verwirklichung zu t1 angegebener Ziele

Systematische Veränderungen im Therapieverlauf können auch mit Hilfe der Selbsteinschätzung der Studienteilnehmer bezüglich ihrer zu Therapiebeginn genannten Probleme und Ziele überprüft werden. Dabei beurteilen sie wiederholt ihre anfangs formulierten Angaben zu nachfolgenden Befragungszeitpunkten, so dass die Entwicklung des Leidensdrucks sowie der Zielverwirklichung aus Sicht der Patienten selbst (ohne weitere Veränderung durch Ratings) dargestellt werden kann. Die Datenauswahl zugunsten der Angaben aus der ersten Befragung wurde gewählt, um einen möglichst langen Verlauf verfolgen zu können.

Zuerst werden alle zu t1 von den Patienten formulierten Problem- und Zielangaben im Verlauf analysiert, bevor die Kategorien „Angst“/ "Angst bewältigen“ sowie „körperliche Beschwerden“/ „körperliche Beschwerden bessern“ exemplarisch näher betrachtet werden. Im Rahmen der Analyse dieser Daten wurde ein Signifikanztest hinsichtlich der Unterschiede von Leidensdruck unter den Problemen und der Verwirklichung der Ziele zu einzelnen Zeitpunkten (t1, t2, t3 und t5) vorgenommen. Außerdem wurde anhand dieser Daten ein Vergleich beider Therapiegruppen (VT und SET) erstellt. Um diese Tests bei allen Problemen und Zielen von t1 durchführen zu können, war eine vorherige Indexbildung der Werte des Leidensdrucks bzw. des ZielErreichens jedes Patienten zu den untersuchten Befragungszeitpunkten nötig. Hierzu 
wurde jeweils der Median des angegebenen Leidensdrucks und des Maßes an Zielerreichen der Problem- und Zielangaben eines jeden Patienten zu den jeweils untersuchten Zeitpunkten berechnet. Fehlende Angaben wurden mit der Berechnung des Medians berücksichtigt. Bei der exemplarischen Analyse einzelner Kategorien wurde der Median der Selbsteinschätzungswerte nur bei Studienteilnehmern verwendet, welche zu t1 mehr als ein Problem oder Ziel der entsprechenden Kategorie nannten. Zur Analyse des Zielerreichens ist zu erwähnen, dass diese Angaben erst ab Befragungszeitpunkt t2 (12. Therapiesitzung) vorliegen. Ob ein zu t1 (3. Therapiesitzung) genanntes Ziel erreicht wurde, lässt sich frühestens einen Befragungszeitpunkt später beurteilen, somit sind zu t2 die ersten Werte des Zielerreichens vorhanden.

Ein Vergleich der Werte des Leidensdrucks bzw. der Zielverwirklichung zu t1 gemachter Angaben zwischen den verschiedenen Zeitpunkten sowie ein Vergleich der Selbsteinschätzungen zwischen beiden Therapiegruppen zu den einzelnen Zeitpunkten wurde mittels statistischer Tests durchgeführt (Sign-Test und Mann-Whitney-UTest, nähere Angaben zur statistischen Auswertung unter 2.2.3 und 7.3.2). Da Selbsteinschätzungswerte verschiedener Zeitpunkte miteinander verglichen werden, konnten nur Angaben von Patienten berücksichtigt werden, die zu diesen Zeitpunkten vorlagen, was eine kleinere Anzahl als $n=51$ der untersuchten Patienten erklärt. Um eine zu hohe Anzahl falsch positiver Testentscheidungen zu vermeiden, wurden die p-Werte gemäß Bonferroni adjustiert, so dass Testergebnisse mit einem Signifikanzniveau von alpha $=0,05 / 3=0,017$ als signifikant betrachtet werden können.

\section{Auswertung aller Problem- und Zielangaben des Zeitpunktes t1}

Leidensdruck unter den zu Therapiebeginn angegebenen Problemen im Verlauf:

Die Auswertung ergibt, dass der Leidensdruck im Therapiezeitraum selbst (t1 bis $\mathrm{t} 3$ ) sowie im Vergleich der Werte von Therapiebeginn mit der Einjahres-Katamnese (t5), signifikant sinkt (jeweils $p=<0,001$ ): Zu t1 beträgt der Median des Leidensdrucks 3 („stark“), während er zu t3 und t5 nur noch bei 1 (, wenig“) liegt. Zwischen Therapieende und der Befragung ein Jahr danach (t5) kommt es zu keiner weiteren signifikanten Änderung des Leidensdrucks ( $p=0,606)$, er bleibt im Median bei 1 .

Tabelle 11: Signifikanztest der Abnahme des Leidensdrucks, gesamtes Patientenkollektiv 
Sign-Test bezüglich der Reduktion des Leidensdrucks unter zu t1 formulierten Problemen, gesamtes

Patientenkollektiv; Vergleich verschiedener Zeitpunkte, signifikant ab $p<0,017$

\begin{tabular}{|c|c|c|c|c|}
\hline $\begin{array}{c}\text { Median } \\
\text { Leidensdruck }\end{array}$ & $\begin{array}{c}\text { verglichene } \\
\text { Zeitpunkte }\end{array}$ & $\begin{array}{c}\text { Anzahl n Patienten, die zu den jeweils vergli- } \\
\text { chenen Zeitpunkten Leidensdruck } \\
\text { angaben }\end{array}$ & $\mathrm{p}$ & $\begin{array}{c}\text { Signifikanz der } \\
\text { Reduktion des } \\
\text { Leidensdrucks? }\end{array}$ \\
\hline $\mathrm{t} 1=3$ & $\mathrm{t} 1$ mit t3 & 36 & $<0,001$ & ja \\
\cline { 2 - 5 } $\mathrm{t} 3=1$ & $\mathrm{t} 1$ mit t5 & 30 & $<0,001$ & ja \\
\cline { 2 - 5 } $\mathrm{t} 5=1$ & $\mathrm{t} 3$ mit t5 & 28 & 0,606 & nein \\
\hline
\end{tabular}

Der Therapiegruppenvergleich zeigt, dass sich zum Zeitpunkt $\mathrm{t} 1$ beide Vergleichsgruppen hinsichtlich der Stärke des Leidensdrucks unter zu t1 angegebenen Problemen unterscheiden, was sich im Signifkanztest als $p_{t 1}=0,016$ niederschlägt. Das arithmetische Mittel des Leidensdrucks ist in der Gruppe verhaltenstherapeutisch behandelter Patienten zu t1 höher (Mittelwert 3,29) als in der psychodynamisch therapierten Vergleichsgruppe (Mittelwert 2,92), die Mediane beider Vergleichsgruppen sind identisch. Zu den Zeitpunkten $\mathrm{t} 3$ und t5 kann kein signifikanter Unterschied der Leidensdruck-Intensität unter zu t1 genannter Problemen festgestellt werden $\left(p_{\mathrm{t} 3}=\right.$ $\left.0,129, p_{\mathrm{t} 5}=0,707\right)$.

Tabelle 12: Signifikanztest des Unterschiedes der Leidensdruck-Intensität, Vergleich beider Therapiegruppen

Zum Zeitpunkt $\mathrm{t} 1$ ist der Leidensdruck in beiden Therapiegruppen zwar im Median gleich, dennoch zeigt die Auswertung aller Einzelwerte beider Gruppen einen signifikanten Unterschied (arithmetisches Mittel Leidensdruck VT 3,29, SET 2,92). Zum Zeitpunkt t3 ist es umgekehrt: Zwar sind die Mediane des Leidensdrucks beider Gruppen unterschiedlich, jedoch zeigte sich in der Auswertung keine signifikante Differenz. Dies kann darin begründet sein, dass der Stichprobenumfang zu klein ist, um den Unterschied aufzudecken, oder der Effekt nur sehr klein, bzw. die Varianz in den Gruppen zu groß ist.

Mann-Whitney-U-Test für Vergleich des Leidensdrucks beider Therapiegruppen unter zu t1 formulierten Problemen; Vergleich zu t1, t3 und t5, signifikant ab $p<0,017$

\begin{tabular}{|c|c|c|c|c|c|c|}
\hline $\begin{array}{c}\text { Zeit- } \\
\text { punkt }\end{array}$ & \multicolumn{2}{|c|}{$\begin{array}{c}\text { Median } \\
\text { Leidensdruck }\end{array}$} & \begin{tabular}{c} 
Anzahl n VT- \\
Patienten, die zum \\
jeweiligen Zeitpunkt \\
\cline { 2 - 3 }
\end{tabular} & $\begin{array}{c}\text { Anzahl n SET- } \\
\text { Patienten, die zum } \\
\text { Leidensdruck angaben }\end{array}$ & $\begin{array}{c}\mathrm{p} \\
\text { Leidensdruck angaben }\end{array}$ & $\begin{array}{c}\text { Signifikanz im Unter- } \\
\text { schied des Leidens- } \\
\text { drucks beider } \\
\text { Therapiegruppen? }\end{array}$ \\
\hline $\mathrm{t} 1$ & 3 & 3 & 24 & 24 & 0,016 & ja \\
\hline $\mathrm{t} 3$ & 1 & 2 & 21 & 18 & 0,129 & nein \\
\hline $\mathrm{t} 5$ & 1 & 1 & 20 & 13 & 0,707 & nein \\
\hline
\end{tabular}

\section{Zielverwirklichung der zu Therapiebeginn formulierten Ziele im Verlauf:}


Das Maß an Zielverwirklichung zeigt einen signifikanten Unterschied zwischen den Befragungszeitpunkten t2 und t5, der Median verändert sich von 2 („wenig“) auf 3 („viel“, $p=0,009)$. Der Vergleich der Zielverwirklichungs-Werte von t2 mit t3 ( $p=$ $0,044)$ sowie $\mathrm{t} 3$ mit $\mathrm{t} 5(\mathrm{p}=0,546)$ zeigt keine signifikanten Unterschiede zwischen den jeweiligen Verteilungen der Leidensdruck-Mediane der Patienten.

Tabelle 13: Signifikanztest der Zunahme des Maßes an Zielverwirklichung, gesamtes Patientenkollektiv

Der Vergleich der Zielverwirklichung von $\mathrm{t} 2$ mit $\mathrm{t} 3$ zeigt trotz Zunahme des Medianes keinen signifikanten Unterschied, da der $p$-Wert aufgrund der Bonferroni-Adjustierung erst ab $<0,017$ als signifikant beurteilt wird.

Sign-Test bezüglich der Steigerung des Erreichens zu t1 formulierter Ziele, gesamtes

Patientenkollektiv; Vergleich verschiedener Zeitpunkte, signifikant ab p $<0,017$

\begin{tabular}{|c|c|c|c|c|}
\hline $\begin{array}{c}\text { Median Ziel- } \\
\text { verwirklichung }\end{array}$ & $\begin{array}{c}\text { verglichene } \\
\text { Zeitpunkte }\end{array}$ & $\begin{array}{c}\text { Anzahl n Patienten, die zu den jeweils } \\
\text { verglichenen Zeitpunkten Maß des Errei- } \\
\text { chens zu t1 genannter Ziele angaben }\end{array}$ & $\mathrm{p}$ & $\begin{array}{c}\text { Signifikanz der Zu- } \\
\text { nahme des Errei- } \\
\text { chens der Ziele? }\end{array}$ \\
\hline \multirow{2}{*}{ 2 $=2$} & $\mathrm{t} 2$ mit t3 & 38 & 0,044 & nein \\
t3 $=3$ & $\mathrm{t} 2$ mit t5 & 31 & 0,009 & ja \\
\cline { 2 - 5 } t5 $=3$ & $\mathrm{t} 3$ mit t5 & 28 & 0,546 & nein \\
\hline
\end{tabular}

Dabei kann zu keinem der überprüften Zeitpunkte zwischen beiden Therapiegruppen ein signifikanter Unterschied im Maß der Zielverwirklichung zu t1 formulierter Ziele festgestellt werden $\left(p_{t 2}=0,132, p_{t 3}=0,216, p_{t 5}=0,496\right)$.

Tabelle 14: Signifikanztest des Unterschiedes im Maß der Zielverwirklichung, Vergleich beider Therapiegruppen

Zwar zeigen sich zu den Zeitpunkten t2 und $\mathrm{t} 3$ Unterschiede Im Median der Zielverwirklichung zwischen beiden Vergleichsgruppen, jedoch sind diese nicht signifikant. Dies kann darin begründet sein, dass der Effekt ist nur sehr klein, die Varianz in den Gruppen zu groß, oder der Stichprobenumfang ist zu klein ist, um den Unterschied aufzudecken.

\begin{tabular}{|c|c|c|c|c|c|c|}
\hline \multicolumn{7}{|c|}{$\begin{array}{l}\text { Mann-Whitney-U-Test für Vergleich beider Therapiegruppen bezüglich des Erreichens der zu t1 formu- } \\
\qquad \text { lierter Ziele; Vergleich zu t2, t3 und t5, signifikant ab } p<0,017\end{array}$} \\
\hline \multirow[t]{2}{*}{$\begin{array}{l}\text { Zeit- } \\
\text { punkt }\end{array}$} & \multicolumn{2}{|c|}{$\begin{array}{c}\text { Median } \\
\text { Zielverwirklichung }\end{array}$} & \multirow{2}{*}{$\begin{array}{l}\text { Anzahl n VT-Patien- } \\
\text { ten, die zum jeweili- } \\
\text { gen Zeitpunkt Lei- } \\
\text { densdruck angaben }\end{array}$} & \multirow{2}{*}{$\begin{array}{l}\text { Anzahl n SET-Patien- } \\
\text { ten, die zum jeweili- } \\
\text { gen Zeitpunkt Lei- } \\
\text { densdruck angaben }\end{array}$} & \multirow[t]{2}{*}{$p$} & \multirow{2}{*}{$\begin{array}{l}\text { Signifikanz im Unter- } \\
\text { schied beider Thera- } \\
\text { pien bezüglich des } \\
\text { Erreichens der Ziele? }\end{array}$} \\
\hline & VT & SET & & & & \\
\hline t2 & 3 & 2 & 23 & 21 & 0,132 & nein \\
\hline t3 & 3 & 2 & 21 & 18 & 0,216 & nein \\
\hline t5 & 3 & 3 & 20 & 13 & 0,496 & nein \\
\hline
\end{tabular}




\section{Exemplarische Darstellung der Kategorien „Angst“ und „Angst bewältigen“ (P3}

\section{und Z3 des Kategoriensystems)}

Die Kategorie „Angst“ hat eine zentrale Bedeutung bei der Untersuchung der Therapieverläufe von Patienten mit GAS, weshalb auch die Selbsteinschätzung derjenigen Patienten, die zu Beginn der Therapie (Befragungszeitpunkt t1) Angst als Problem (32 Patienten), oder deren Bewältigung als Ziel (36 Patienten) nannten, wichtig zur Beurteilung des Therapieverlaufs ist.

\section{Leidensdruck unter zu Therapiebeginn genannter Angst im Verlauf:}

Untersucht wurden die Angaben aller Patienten, die zur 3. Therapiesitzung (Befragungszeitpunkt t1) Angst als Problem aufschrieben und auch im späteren Verlauf Angaben zum Leidensdruck darunter machten. Das Ergebnis zeigt, dass der Median des Leidensdrucks unter diesen Problemen im Therapieverlauf von 3/ „stark“ zu t1 auf $1 /$ „wenig“ zu Therapieende sinkt ( $p=<0,001)$. Auch ein Jahr nach Behandlungsabschluss (Zeitpunkt t5) liegt dieser Wert bei 1 (Vergleich $\mathrm{t} 1$ mit t5 $p=<0,001$ ). Im Vergleich der Befragungszeitpunkte Therapieende (t3) und ein Jahr nach Therapieende (t5) kann somit keine weitere signifikante Senkung des Leidensdrucks beobachtet werden $(p=0,228)$.

Tabelle 15: Signifikanztest der Abnahme des Leidensdrucks unter zu Therapiebeginn angegebener Angst

\begin{tabular}{|c|c|c|c|c|}
\hline \multicolumn{5}{|c|}{$\begin{array}{l}\text { Sign-Test bezüglich der Reduktion des Leidensdrucks unter zu t1 formulierter Angst; } \\
\text { Vergleich verschiedener Zeitpunkte, signifikant ab } p<0,017\end{array}$} \\
\hline $\begin{array}{l}\text { Median } \\
\text { Leidensdruck }\end{array}$ & $\begin{array}{l}\text { verglichene } \\
\text { Zeitpunkte }\end{array}$ & $\begin{array}{l}\text { Anzahl n Patienten, die zu den jeweils } \\
\text { verglichenen Zeitpunkten Leidensdruck } \\
\text { angaben }\end{array}$ & $p$ & $\begin{array}{l}\text { Signifikanz der } \\
\text { Reduktion des } \\
\text { Leidensdrucks? }\end{array}$ \\
\hline$t 1=3$ & t1 mit t3 & 22 & $<0,001$ & ja \\
\hline$t 3=1$ & t1 mit t5 & 21 & $<0,001$ & ja \\
\hline $\mathrm{t} 5=1$ & t3 mit t5 & 18 & 0,228 & nein \\
\hline
\end{tabular}

Der Vergleich der Leidensdruck-Werte der untersuchten Zeitpunkte in beiden Therapiegruppen ergibt keinen signifikanten Unterschied $\left(p_{t 1}=0,062, p_{t 3}=0,792, p_{t 5}=\right.$ $0,795)$. 
Tabelle 16: Signifikanztest des Unterschiedes der Leidensdruck-Intensität unter zu Therapiebeginn angegebener Angst, Vergleich beider Therapiegruppen

Dass die Mediane zu den Zeitpunkten $\mathrm{t} 1$ und $\mathrm{t} 3$ zwischen den Gruppen leicht voneinander abweichen, aber kein signifikanter Unterschied nachweisbar ist, kann daran liegen, dass der Effekt ist nur sehr klein, der Stichprobenumfang zu klein, oder die Varianz in den Gruppen zu groß ist, um den Unterschied aufzudecken.

Mann-Whitney-U-Test für Vergleich des Leidensdrucks beider Therapiegruppen unter zu t1 formulierter Angst; Vergleich zu t1, t3 und t5, signifikant ab $p<0,017$

\begin{tabular}{|c|c|c|c|c|c|c|}
\hline \multirow[t]{2}{*}{$\begin{array}{l}\text { Zeit- } \\
\text { punkt }\end{array}$} & \multicolumn{2}{|c|}{$\begin{array}{c}\text { Median } \\
\text { Leidensdruck }\end{array}$} & \multirow{2}{*}{$\begin{array}{c}\text { Anzahl n VT- } \\
\text { Patienten, die zum } \\
\text { jeweiligen Zeitpunkt } \\
\text { Leidensdruck angaben }\end{array}$} & \multirow{2}{*}{$\begin{array}{c}\text { Anzahl n SET- } \\
\text { Patienten, die zum } \\
\text { jeweiligen Zeitpunkt } \\
\text { Leidensdruck angaben }\end{array}$} & \multirow[t]{2}{*}{$p$} & \multirow{2}{*}{$\begin{array}{l}\text { Signifikanz im Unter- } \\
\text { schied des Leidens- } \\
\text { drucks beider } \\
\text { Therapiegruppen? }\end{array}$} \\
\hline & VT & SET & & & & \\
\hline $\mathrm{t} 1$ & 3,5 & 3 & 17 & 13 & 0,062 & nein \\
\hline t3 & 1,25 & 1 & 16 & 8 & 0,792 & nein \\
\hline t5 & 1 & 1 & 16 & 7 & 0,795 & nein \\
\hline
\end{tabular}

Zielverwirklichung der zu Therapiebeginn formulierten Ziele, Angst bewältigen zu wollen, im Verlauf:

Die Analyse der Selbsteinschätzungsdaten von Patienten, die zu Therapiebeginn Ziele im Zusammenhang mit der Bewältigung von Angst, sowie zu späteren Befragungen Angaben zu deren Verwirklichung machten, zeigt folgende Resultate: Das Ausmaß, in dem diese Ziele erreicht wurden, verändert sich im zeitlichen Verlauf nur tendenziell in Richtung einer Zunahme des Zielverwirklichung, die Veränderungen werden nicht signifikant (Vergleich t2 mit t3 $p=0,043$, Vergleich t2 mit t5 $p=0,181$, Vergleich $\mathrm{t} 3$ mit t5 $p=0,450$ ). Das Niveau befindet sich dabei im Median zwischen $2 /$ "etwas" und $3 /$ "viel".

Tabelle 17: Signifikanztest der Zunahme des Maßes an Zielverwirklichung zu Therapiebeginn genannter Ziele, Angst bewältigen zu wollen

Der Vergleich der Mediane von Zielverwirklichung der Zeitpunkte t2 mit t3 sowie t2 mit t5 zeigt einen Unterschied, dieser muss jedoch aufgrund des Signifikanzniveaus von alpha $=0,017$ (durch die Bonferroni-Adjustierung) als nicht signifikant beurteilt wird.

\begin{tabular}{|c|c|c|c|c|}
\hline \multicolumn{5}{|c|}{$\begin{array}{l}\text { Sign-Test bezüglich der Steigerung des Erreichens zu t1 formulierter Ziele, Angst zu bewältigen; } \\
\qquad \text { Vergleich verschiedener Zeitpunkte, signifikant ab } p<0,017\end{array}$} \\
\hline $\begin{array}{c}\text { Median } \\
\text { Zielver- } \\
\text { wirklichung }\end{array}$ & $\begin{array}{l}\text { verglichene } \\
\text { Zeitpunkte }\end{array}$ & $\begin{array}{c}\text { Anzahl n Patienten, die zu den jeweils } \\
\text { verglichenen Zeitpunkten Maß des Errei- } \\
\text { chens der Ziel angaben }\end{array}$ & $\mathrm{p}$ & $\begin{array}{l}\text { Signifikanz der } \\
\text { Zunahme des Errei- } \\
\text { chens der Ziele? }\end{array}$ \\
\hline $\mathrm{t} 2=2$ & $\mathrm{t} 2$ mit t3 & 25 & 0,043 & nein \\
\hline $\mathrm{t} 3=3$ & t2 mit t5 & 23 & 0,181 & nein \\
\hline $\mathrm{t} 5=3$ & t3 mit t5 & 19 & 0,450 & nein \\
\hline
\end{tabular}


Der Therapiegruppen-Vergleich der Patienten, die zu $\mathrm{t} 1$ das Ziel formulierten, Angst bewältigen zu wollen, lässt zu den überprüften Zeitpunkten ebenfalls keinen signifikanter Unterschied im Maß des Erreichens dieser Ziele erkennen $\left(p_{\mathrm{t} 2}=0,652, p_{\mathrm{t} 3}=\right.$ $\left.0,304, p_{\mathrm{t} 5}=0,667\right)$.

Tabelle 18: Signifikanztest des Unterschiedes im Maß der Zielverwirklichung zu Therapie genannter Ziele, Angst bewältigen zu wollen, Vergleich beider Therapiegruppen

\begin{tabular}{|c|c|c|c|c|c|c|}
\hline \multirow[t]{2}{*}{$\begin{array}{l}\text { Zeit- } \\
\text { punkt }\end{array}$} & \multicolumn{2}{|c|}{$\begin{array}{c}\text { Median Maß } \\
\text { Zielverwirklichung }\end{array}$} & \multirow{2}{*}{$\begin{array}{l}\text { Anzahl n VT-Patien- } \\
\text { ten, die zum jeweili- } \\
\text { gen Zeitpunkt Lei- } \\
\text { densdruck angaben }\end{array}$} & \multirow{2}{*}{$\begin{array}{l}\text { Anzahl n SET-Patien- } \\
\text { ten, die zum jeweili- } \\
\text { gen Zeitpunkt Lei- } \\
\text { densdruck angaben }\end{array}$} & \multirow[t]{2}{*}{$p$} & \multirow{2}{*}{$\begin{array}{l}\text { Signifikanz im Unter- } \\
\text { schied beider Thera- } \\
\text { pien bezüglich des } \\
\text { Erreichens der Ziele? }\end{array}$} \\
\hline & VT & SET & & & & \\
\hline t2 & 2 & 2 & 18 & 13 & 0,652 & nein \\
\hline t3 & 3 & 3 & 15 & 11 & 0,304 & nein \\
\hline t5 & 3 & 3 & 16 & 9 & 0,667 & nein \\
\hline
\end{tabular}

Exemplarische Darstellung der Kategorien „körperliche Beschwerden“ und „körperliche Beschwerden bessern“ (P1 und Z1 des Kategoriensystems)

Eine weitere Beispiel-Kategorie, die sich in der Anzahl der genannten Probleme und Ziele systematisch verändert, ist die der „körperlichen Beschwerden“ (Kategorie 1 des Kategoriensystems). Es wurden wiederum alle Patienten einbezogen, die zum Befragungszeitpunkt t1 Probleme (18 Patienten) oder Ziele (16 Patienten) formulierten, die körperliche Beschwerden beinhalteten. Ausgewertet werden konnten Angaben von Patienten, die durch Selbsteinschätzung den Leidensdruck oder das Erreichen dieser Ziele wiederholt beurteilten.

Leidensdruck unter den zu Therapiebeginn genannten körperlichen Beschwerden im Verlauf:

Der Median des Leidensdrucks unter anfangs angegebenen körperlichen Beschwerden sinkt signifikant von 3/ „stark“ zu t1 bis auf $1 /$ „wenig“ zu Therapieende ( $p=$ 0,004). Dieses Niveau wird bis zur Katamnese t5 ein Jahr nach Behandlungsabschluss unverändert beibehalten (Vergleich des Leidensdrucks von t1 mit t5 $p=$ $0,001$, Vergleich $\mathrm{t} 3$ mit $\mathrm{t} 5 \mathrm{p}=1,00)$. 
Tabelle 19: Signifikanztest der Abnahme des Leidensdrucks unter zu Therapiebeginn angegebenen körperlichen Beschwerden

Sign-Test bezüglich der Reduktion des Leidensdrucks unter zu t1 formulierten körperlichen Beschwerden; Vergleich verschiedener Zeitpunkte, signifikant ab $p<0,017$

\begin{tabular}{|c|c|c|c|c|}
\hline $\begin{array}{c}\text { Median } \\
\text { Leidensdruck }\end{array}$ & $\begin{array}{l}\text { verglichene } \\
\text { Zeitpunkte }\end{array}$ & $\begin{array}{c}\text { Anzahl n Patienten, die zu den jeweils } \\
\text { verglichenen Zeitpunkten } \\
\text { Leidensdruck angaben }\end{array}$ & $p$ & $\begin{array}{l}\text { Signifikanz der } \\
\text { Reduktion des } \\
\text { Leidensdrucks? }\end{array}$ \\
\hline $\mathrm{t} 1=3$ & $\mathrm{t} 1 \mathrm{mit} \mathrm{t} 3$ & 12 & 0,004 & ja \\
\hline $\mathrm{t} 3=1$ & t1 mit t5 & 14 & 0,001 & ja \\
\hline $\mathrm{t} 5=1$ & t3 mit t5 & 12 & 1,000 & nein \\
\hline
\end{tabular}

Die separate Untersuchung der beiden Vergleichsgruppen hinsichtlich möglicher Unterschiede in der Intensität des Leidensdrucks zeigt, dass sich diese zu keinem der untersuchten Zeitpunkte signifikant voneinander unterscheiden $\left(p_{\mathrm{t} 1}=0,825, p_{\mathrm{t} 3}=\right.$ $\left.0,257, p_{t 5}=0,017\right)$.

Tabelle 20: Signifikanztest des Unterschiedes der Leidensdruck-Intensität unter zu Therapiebeginn angegebenen körperlichen Beschwerden, Vergleich beider Therapiegruppen

Es besteht zu den Zeitpunkten $\mathrm{t} 3$ und $\mathrm{t} 5$ jeweils kein signifikanter Unterschied in der Stärke des Leidensdrucks zwischen beiden Therapiegruppen, obwohl sich die Mediane des Leidensdrucks voneinander unterscheiden. Dies kann folgende Gründe haben: Der Effekt ist nur sehr klein, die Varianz in den Gruppen ist zu groß oder der Stichprobenumfang ist zu klein, um den Unterschied aufzudecken.

Mann-Whitney-U-Test für Vergleich des Leidensdrucks beider Therapiegruppen unter zu t1 formulierten
körperlichen Beschwerden; Vergleich zu t1, t3 und t5, signifikant ab p $\mathbf{0 , 0 1 7}$

Zielverwirklichung der zu Therapiebeginn formulierten Ziele, körperliche Beschwerden bessern zu wollen, im Verlauf:

Das Ausmaß, in dem zu t1 formulierte Ziele, körperliche Beschwerden zu bessern erreicht wurden, verändert sich im zeitlichen Verlauf in der Gesamtgruppe der Patienten im Median nicht signifikant. Dieser liegt durchgängig von Befragungszeitpunkt t2 (12. Therapiesitzung) bis t5 (ein Jahr nach Therapieende) auf einem Niveau von $3 /$ 
Ziele „viel“ erreicht. Das Ergebnis wird durch den Signifikanztest bestätigt (Vergleich t2 mit t3 $p=0,248$, Vergleich t2 mit t5 $p=0,074$, Vergleich t3 mit t5 $p=0,248$ ).

Tabelle 21: Signifikanztest der Zunahme des Maßes an Zielverwirklichung zu Therapiebeginn genannter Ziele, körperliche Beschwerden bessern zu wollen

\begin{tabular}{|c|c|c|c|c|}
\hline \multicolumn{5}{|c|}{$\begin{array}{l}\text { Sign-Test bezüglich der Steigerung des Erreichens zu t1 formulierter Ziele, körperliche } \\
\text { Beschwerden zu bessern; Vergleich verschiedener Zeitpunkte, signifikant ab } p<0,017\end{array}$} \\
\hline $\begin{array}{l}\text { Median Ziel- } \\
\text { verwirklichung }\end{array}$ & $\begin{array}{l}\text { verglichene } \\
\text { Zeitpunkte }\end{array}$ & $\begin{array}{l}\text { Anzahl n Patienten, die zu den jeweils } \\
\text { verglichenen Zeitpunkten Maß des } \\
\text { Erreichens der Ziele angaben }\end{array}$ & $p$ & $\begin{array}{l}\text { Signifikanz der Zunahme } \\
\text { des Erreichens der Ziele? }\end{array}$ \\
\hline $\mathrm{t} 2=3$ & t2 mit t3 & 10 & 0,248 & nein \\
\hline$t 3=3$ & t2 mit t5 & 9 & 0,074 & nein \\
\hline $\mathrm{t} 5=3$ & t3 mit t5 & 9 & 0,248 & nein \\
\hline
\end{tabular}

Der Vergleich der Patienten beider Therapiegruppen, die zu t1 das Ziel formulierten, körperliche Beschwerden zu bessern zeigt, dass zu den drei untersuchten Befragungszeitpunkten zwischen beiden Therapiegruppen kein signifikanter Unterschied im Maß des Erreichens dieser Ziele besteht $\left(p_{t 2}=0,762, p_{t 3}=0,071, p_{t 5}=0,233\right)$.

Tabelle 22: Signifikanztest des Unterschiedes im Maß der Zielverwirklichung zu Therapie genannter Ziele, körperliche Beschwerden bessern zu wollen; Vergleich beider Therapiegruppen

Es besteht zu den Zeitpunkten $\mathrm{t} 2$ und $\mathrm{t} 3$ kein signifikanter Unterschied im Maß des Zielerreichens zwischen beiden Therapiegruppen, obwohl sich die Mediane beider Gruppen zu diesen Zeitpunkten jeweils voneinander unterscheiden (VT 3, SET 2,5). Dies kann folgende Gründe haben: Der Effekt ist nur sehr klein, die Varianz in den Gruppen ist zu groß oder der Stichprobenumfang ist zu klein, um den Unterschied aufzudecken.

Mann-Whitney-U-Test für Vergleich beider Therapiegruppen bezüglich des Erreichens zu t1 formulierter Ziele, körperliche Beschwerden zu bessern; Vergleich zu t2, t3 und t5, signifikant ab p $<0,017$

\begin{tabular}{|c|c|c|c|c|c|c|}
\hline $\begin{array}{c}\text { Zeit- } \\
\text { punkt }\end{array}$ & $\begin{array}{c}\text { Median Maß } \\
\text { Zielverwirklichung }\end{array}$ & \begin{tabular}{c} 
Anzahl n VT- \\
Patienten, die zum \\
jeweiligen Zeitpunkt \\
\cline { 2 - 3 }
\end{tabular} & $\begin{array}{c}\text { Anzahl n SET- } \\
\text { Patienten, die zum } \\
\text { jeidensdruck angaben } \\
\text { jeiligen Zeitpunkt } \\
\text { Leidensdruck angaben }\end{array}$ & SET & $\mathrm{p}$ & $\begin{array}{c}\text { Signifikanz im Unter- } \\
\text { schied beider Thera- } \\
\text { pien bezüglich des } \\
\text { Erreichens der } \\
\text { Ziele? }\end{array}$ \\
\hline t2 & 3 & 2,5 & 5 & 6 & 0,762 & nein \\
\hline t3 & 3 & 2,5 & 5 & 6 & 0,071 & nein \\
\hline t5 & 3 & 3 & 5 & 5 & 0,233 & nein \\
\hline
\end{tabular}


Fazit:

Die untersuchte Hypothese, dass es im Therapieverlauf zu systematischen Veränderungen kommt, kann bestätigt werden. Es wurde festgestellt, dass sich diese Änderungen in vielfältiger Weise darstellen lassen.

Demnach zeigt die Auswertung mittels des Kategoriensystems, dass Kategorien wie „körperliche Beschwerden“, „Angst“ oder „depressive Beschwerden“ im Therapieverlauf seltener, dafür z.B. Alltagsprobleme häufiger genannt werden. Die Prüfung auf systematische Veränderungen der Problemwahrnehmung und -bewältigung mit Hilfe der Mastery-Awareness-Scale lässt erkennen, dass Probleme im Laufe der Behandlung immer seltener als „nicht greifbar“ und häufiger als "vage“ erlebt werden. Die Bewältigungsmöglichkeiten verändern sich im Sinne einer abnehmenden Häufigkeit von Hilflosigkeit hin zu mehr Einflussmöglichkeiten im Umgang mit angegebenen Problemen. Schließlich wird auch anhand der Selbsteinschätzungsdaten bezüglich des Leidensdrucks unter zu Befragungszeitpunkt $\mathbf{t} 1$ genannten Problemen und der Umsetzung zu t1 angegebener Ziele deutlich, dass der Leidensdruck im Therapiezeitraum sinkt und die Verwirklichung zu t1 angegebener Ziele zeitweise steigt oder auf hohem Niveau bleibt. Hervorzuheben sind hier die Verläufe der Kategorien „Angst“ und „körperliche Beschwerden“, welche im Verlauf signifikant weniger Leidensdruck verursachen. Die Ergebnisse der Selbsteinschätzungen der Patienten konnten im Rahmen dieser Arbeit hinsichtlich ihrer statistischen Signifikanz geprüft und bestätigt werden.

\subsection{Prüfung von Hypothese H2.1}

„Es gibt Prädiktoren, die für einen guten oder eher mäßigen Therapieverlauf sprechen."

Zum besseren Verständnis der Hypothesen H2.1 und H2.2 wird an dieser Stelle kurz auf den Begriff ,Prädiktor‘ eingegangen. Hierbei handelt es sich um eine zur Vorhersage bestimmter Ereignisse oder Gegebenheiten benutze Variable, eine sogenannte Vorhersagevariable (Häcker und Stapf 2004). Es wird also bei der Überprüfung der beiden folgenden Hypothesen untersucht, ob es seitens der Studienteilnehmer Variablen gibt, welche möglicherweise einen guten oder eher mäßigen Therapieverlauf 
begünstigen könnten. Dies könnten z.B. biographische Merkmale oder bestimmte Inhalte in den Problem- und Zielangaben der Patienten sein. Die Identifikation eines möglichen Prädiktors im Sinne einer Vorhersagevariable bedeutet nicht zwangsläufig, dass alle Patienten, auf die dieser Prädiktor zutrifft, auch ein bestimmtes Therapieergebnis erreichen. Die Erkenntnisse der vorliegenden Hypothesenprüfung sollten daher als Tendenzen verstanden werden. Desweiteren gilt es zu berücksichtigen, dass sie im Rahmen der vorliegenden Arbeit keinen Signifikanztests unterzogen wurden.

Prädiktoren wurden in den Angaben der Studienteilnehmer von Therapiebeginn (Befragungszeitpunkt t1, 3. Therapiesitzung) gesucht. Ihre Auswirkungen wurden im weiteren Therapieverlauf analysiert, indem die Leidensdruck-Selbsteinschätzungen der Patienten (unter ihren zu t1 genannten Problemen) von Therapiebeginn mit denen von Therapieende ( $\mathrm{t} 3$ ) verglichen wurden. Diese Form der Beurteilung des Behandlungszeitraumes ist möglich, da die Patienten in den PATH-Fragebögen jeweils die von ihnen zu t1 genannten Probleme bezüglich des verursachten Leidensdrucks zu jedem Befragungszeitpunkt mit einer Zahl beurteilen ( $0=$ überhaupt nicht, $1=$ wenig, 2 = etwas, 3 = stark, 4 = könnte nicht schlimmer sein). Zur Überprüfung der Hypothesen $\mathrm{H} 2.1$ und H2.2 konnten folglich nur Daten von Patienten verwendet werden, die einerseits zum Zeitpunkt $\mathrm{t} 1$ mindestens ein Problem nannten und andererseits zu den Erhebungszeitpunkten $\mathrm{t} 1$ und $\mathrm{t} 3$ Angaben bezüglich des Leidensdrucks unter diesen Problemen machten. Die Aussagen, keine Probleme oder Ziele zu haben, kommen in den zur Prüfung der Hypothesen H2.1 und H2.2 untersuchten Formulierungen der Patienten nicht vor.

Die Studienteilnehmer wurden aufgrund der Veränderungen ihres in den PATHFragebögen angegebenen Leidensdrucks in Gruppen eingeteilt: Nach der Senkung der Mediane des Leidensdrucks unter zu Beginn der Therapie angegebenen Problemen von Erhebungszeitpunkt t1 zu t3 wurden drei Gruppen (im Folgenden Gruppe 1 bis 3 ) gebildet, die beste Gruppe (Gruppe 1) weist dabei die größte Differenz/ den stärksten Rückgang im Leidensdruck von t1 zu t3 auf. Dementsprechend ist ein Therapieverlauf umso besser, je deutlicher der Leidensdruck zwischen den zwei Erhebungszeitpunkten reduziert werden konnte.

- Gruppe 1 = guter Therapieverlauf (12 Patienten) 
- Gruppe 2 = mittlerer Therapieverlauf (11 Patienten)

- Gruppe 3 = mäßiger Therapieverlauf (13 Patienten)

Bei der Skalierung, ab welcher Abnahme des Leidensdruck-Medians eine Gruppenzuordnung erfolgte, wurde auf eine möglichst gleichmäßige Verteilung auf die drei Gruppen (guter, mittlerer und mäßiger Erfolg) geachtet. Patienten mit mittlerem Verlauf (Abnahme um ein bis zwei Median Werte) wurden aufgrund ihrer hohen Zahl gesondert den drei Verlaufsgruppen zugeordnet, indem ein Summenscore der Leidensdruck-Werte der einzelnen Probleme gebildet wurde.

Da sich die Therapien insgesamt (gemessen wie hier am Rückgang des Leidensdrucks und anderen Instrumenten) als überwiegend erfolgreich zeigten, liegt eine geringe Varianz der Verläufe vor: Bei allen Patienten, bei denen der Median des Leidensdrucks unter zu Befragungszeitpunkt $\mathrm{t} 1$ formulierten Problemen von $\mathrm{t} 1$ mit $\mathrm{t} 3$ verglichen werden konnte, sinkt dieser oder bleibt selten unverändert (keine Abnahme des Leidensdruck-Medians). Dies erschwert die Bestimmung von Prädiktoren für günstige und weniger günstige Behandlungsverläufe und relativiert möglicherweise eine Zuordnung zur Gruppe mit mäßigem Therapieverlauf (Gruppe 3), denn auch hier konnte der Leidensdruck gesenkt werden, allerdings nicht so stark wie in Gruppe 1 oder 2.

Nachdem die Einteilung der einzelnen Gruppen erfolgt war, konnten die Daten der Patienten im Hinblick auf bestimmte Prädiktoren zum Zeitpunkt t1 (3. Sitzung der Therapie) untersucht und verglichen werden. Dabei wurde sich verschiedener Möglichkeiten bedient:

- Die Patienten wurden einzeln hinsichtlich soziodemographischer Daten analysiert und die Häufigkeit dieser Merkmale in den drei Gruppen berechnet.

- Mit dem Kategoriensystem konnten die Patienten-Aussagen von t1 inhaltlich ausgewertet werden und es wurde untersucht, ob bestimmte Probleme oder Ziele in einer Gruppe häufiger oder seltener vorkommen und somit potentiell für einen erfolgreichen oder weniger erfolgreichen Therapieverlauf sprechen. 
- Auch mit der Mastery-Awareness-Scale kann die Entwicklung einer Therapie bewertet werden. Dazu wurde mit diesem Instrument ebenfalls in den drei Gruppen nach Prädiktoren für den jeweiligen Therapieverlauf gesucht.

- Schließlich wurde der Leidensduck unter den zu Befragungszeitpunkt $\mathrm{t} 1$ angegebenen Problemen zu diesem Zeitpunkt für jeden Patienten ermittelt und berechnet, wie hoch der Median dieser Daten in jeder Gruppe ist.

Es sei erneut daran erinnert, dass mit Mastery-Awareness-Scale und Kategoriensystem Probleme bzw. Ziele bestimmten Kategorien zugeordnet wurden. Hierbei wurden alle von den zu vergleichenden Patienten angegebenen Probleme und Ziele berücksichtigt. Da jeder Patient zum Zeitpunkt 11 (wie auch zu allen anderen Befragungszeitpunkten) angehalten war, jeweils (bis zu) drei Probleme und Ziele anzugeben, waren hier Mehrfachnennungen üblich, was in der absoluten Häufigkeit (wie bei allen Ratings der PATH-Daten mit diesen Instrumenten) z.T. höhere Werte, als Patienten in einer Gruppe sind, ergab. Um diese Tatsache zu berücksichtigen, beschreiben die angegebenen Prozentsätze jeweils den Anteil einer Kategorie an allen Problem- oder Zielangaben der jeweiligen Gruppe zu einem Zeitpunkt (hier t1).

Wegen der fehlenden Intervallskalierung und der Abhängigkeitsstruktur der Messungen zur Darstellung der Unterschiede zwischen den verschiedenen Messzeitpunkten wurden die Daten zur Überprüfung dieser Hypothese deskriptiv ausgewertet.

Weitere Tabellen, welche zusätzliche Prozentsätze wiedergeben, befinden sich im Anhang unter 7.4 .

\subsubsection{Allgemeine Prädiktoren verschiedener Therapieverläufe}

Jüngeres Alter als möglicher Prädiktor eines guten Therapieverlaufs:

Bezüglich des Alters der Patienten zu Therapiebeginn wurde herausgefunden, dass je jünger diese sind, desto besser der Therapieverlauf zu sein scheint. In Gruppe 1 (guter Therapieverlauf) liegt das Durchschnittsalter bei 40 Jahren (arithmetisches Mittel, SD = 13), in Gruppe 2 bei 43 Jahren (arithmetisches Mittel, SD = 8) und in Gruppe 3 bei 48 Jahren (arithmetisches Mittel, SD = 12).

\section{Therapieverfahren als mögliche Prädiktoren:}

Die Beurteilung des Therapieverlaufs von $\mathrm{t} 1$ bis $\mathrm{t} 3$ zeigt, dass der Anteil von verhal- 
tenstherapeutisch behandelten Patienten in Gruppe 1 (guter Therapieverlauf) mit 83,33\% am höchsten ist, in Gruppe 2 beträgt er 45,45\% und in Gruppe 3 30,77\%. Psychodynamisch (also nach SET) therapierte Personen machen in Gruppe 1 16,67\%, in Gruppe $254,55 \%$ und in Gruppe 3 69,23\% aus.

Im Rahmen unseres gewählten Untersuchungsansatzes kann VT, basierend auf der Selbsteinschätzung des Leidensdrucks der Patienten und dessen Abnahme, als möglicher Prädiktor eines guten Therapieverlaufs und SET eher als Prädiktor eines mittleren bis mäßigen Therapieerfolges angenommen werden. Die Interpretation dieser Ergebnisse wirft methodische Fragestellungen auf, die unter 4.3.5 erörtert werden.

Höherer Leidensdruck unter zu t1 formulierten Problemen zum Zeitpunkt t1 als möglicher Prädiktor eines guten Therapieverlaufs:

Der Vergleich der Erfolgsgruppen bezüglich des Leidensdrucks unter zu t1 angegebenen Problemen zu diesem Zeitpunkt zeigt, dass hier ein hoher Leidensdruck Prädiktor eines guten Therapieergebnisses sein könnte: Je höher der LeidensdruckMedian unter den zu $\mathrm{t} 1$ aufgeschriebenen Problemen zu diesem Erhebungszeitpunkt ist, umso erfolgreicher ist die Gruppe bezüglich der Abnahme des Leidensdrucks im weiteren Verlauf der Behandlung. In Gruppe 1 beträgt der Leidensdrucks im Median 3,5, in Gruppe 2 und 3 liegt dieser bei einem Wert von 3.

\subsubsection{Inhaltskategorien des Kategoriensystems als Prädiktoren verschiedener Therapieverläufe}

Eine Übersicht über die Verteilung der zum Befragungszeitpunkt $\mathrm{t1}$ (3. Therapiesitzung) angegebenen Probleme und Ziele auf Kategorien des Kategoriensystems innerhalb der drei Erfolgsgruppen sollen folgende Kreisdiagramme vermitteln. Dabei entsprechen $100 \%$ allen zu $\mathrm{t} 1$ gemachten Problem- oder Zielangaben einer Erfolgsgruppe. 
Abbildung 30: Verteilung zu t1 angegebener Probleme und Ziele der drei Erfolgsgruppen auf die Inhaltskategorien des Kategoriensystems

\section{Probleme Gruppe 1 (guter Therapieverlauf)}

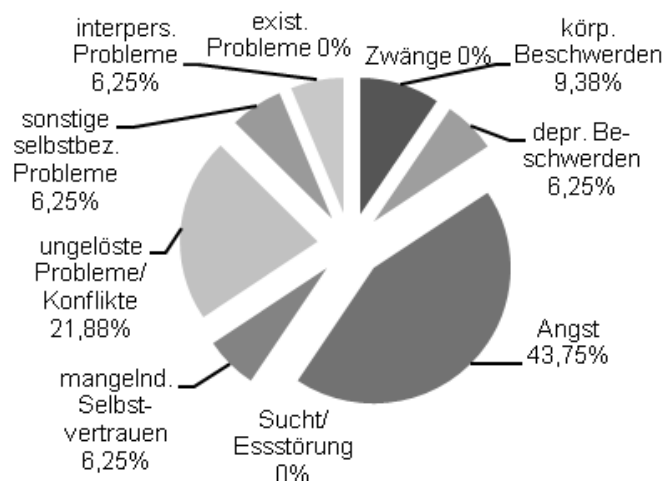

Probleme Gruppe 2 (mittlerer Therapieverlauf)

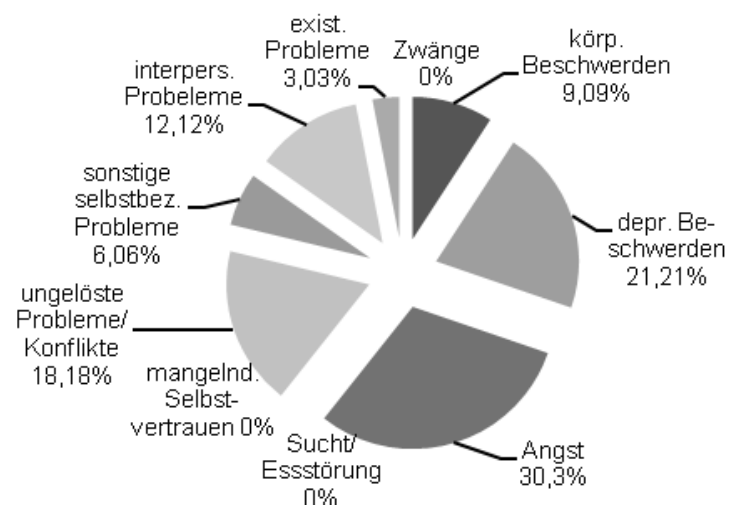

Probleme Gruppe 3 (mäßiger Therapieverlauf)

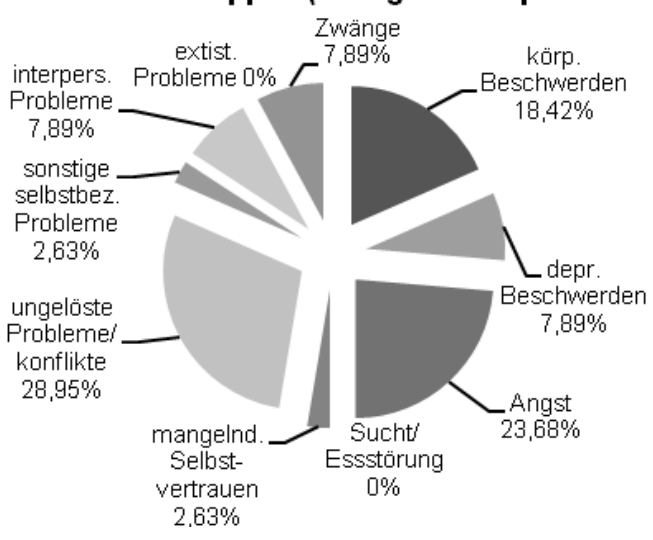

\section{Ziele Gruppe 1 (guter Therapieverlauf)}

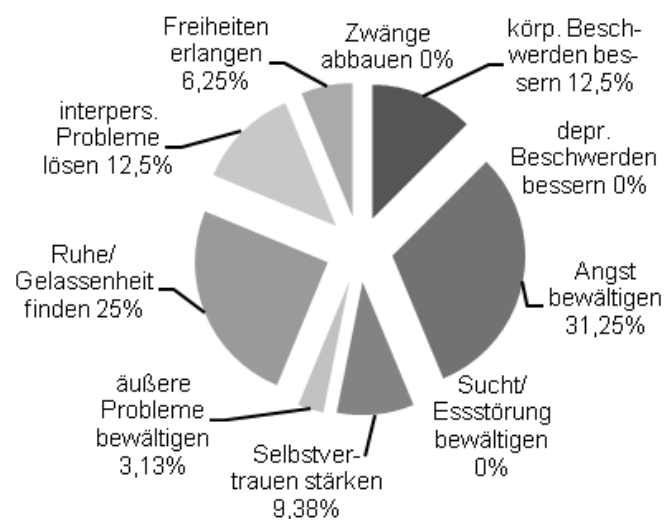

Ziele Gruppe 2 (mittlerer Therapieverlauf)

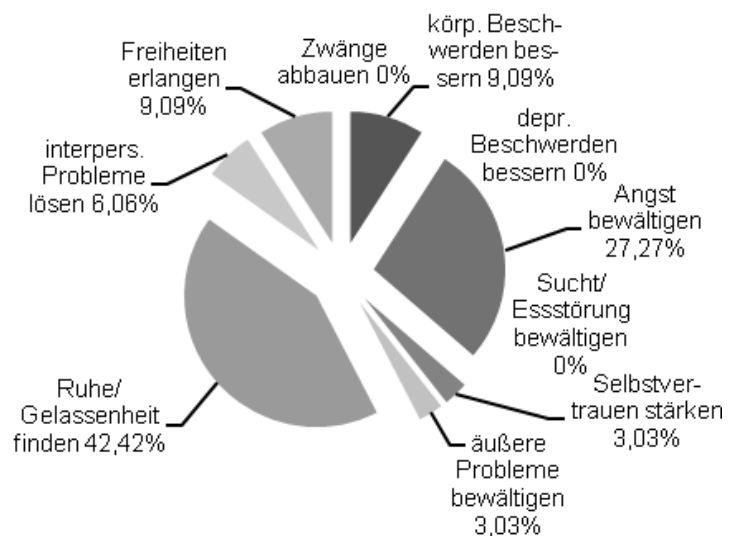

Ziele Gruppe 3 (mäßiger Therapieverlauf)

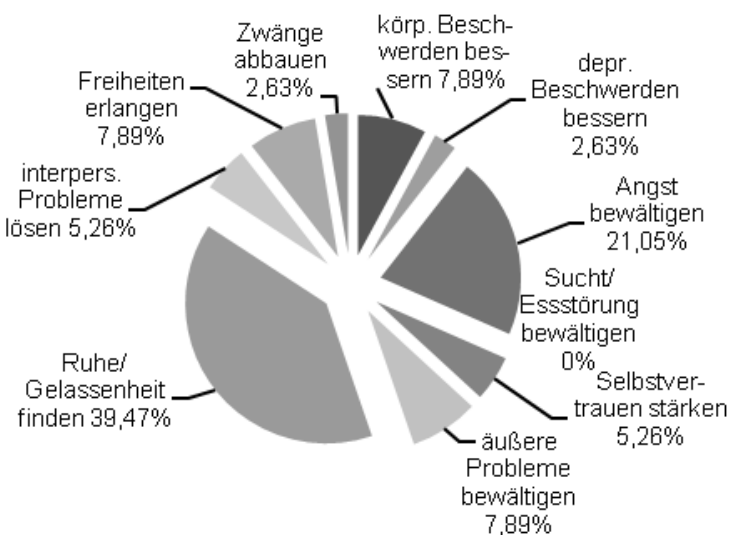

Problem- und Zielinhalte als mögliche Prädiktoren eines guten Therapieverlaufs:

Probleme im Zusammenhang mit Angst (Kategorie P3 Kategoriensystem) wurden zum Befragungszeitpunkt $\mathrm{t} 1$ in Gruppe 1 (guter Therapieerfolg) am häufigsten genannt (hier 43,75\% der Problemangaben, in Gruppe 2 30,3\% und in Gruppe 3 $23,68 \%$ der Probleme) und könnten somit Prädiktor für eine erfolgreiche Psychotherapie der GAS sein. 
Auch Ziele, Angst zu bewältigen (Kategorie Z3) werden zum Zeitpunkt t1 in Gruppe 1 mit gutem Therapieverlauf am häufigsten genannt. Hier beträgt der Prozentsatz an allen zu t1 genannten Zielen dieser Gruppe 31,25\%, in Gruppe 2 27,27\% und in Gruppe 3 21,05\%. Darüber hinaus werden Ziele, körperliche Beschwerden zu bessern (Kategorie Z1) mit 12,5\% der Zielangaben in der erfolgreichsten Gruppe $1 \mathrm{zu}$ diesem Zeitpunkt häufiger als in Gruppe 2 oder 3 angegeben. Diese Zielinhalte können somit ebenfalls als mögliche Prädiktoren einer erfolgreichen Psychotherapie bei GAS gelten.

Problem- und Zielinhalte als mögliche Prädiktoren eines mäßigen Therapieverlaufs: Problemangaben bezüglich körperlicher Beschwerden (Kategorie P1 des Kategoriensystems) werden zu Erhebungszeitpunkt $\mathrm{t} 1$ anteilig häufiger in der Gruppe mit mäßigem Therapieerfolg beschrieben (Gruppe 3 18,42\%, Gruppe 1 und 2 jeweils rund $9 \%$ der in der Gruppe angegebenen Probleme), sie können folglich als Prädiktoren eines eher mäßigen Therapieverlaufs gesehen werden.

Ziele, Ruhe und Gelassenheit zu finden (Kategorie Z7) werden in der mittel und mäBig erfolgreichen Gruppe zum Zeitpunkt t1 anteilig häufiger als in der erfolgreichen Gruppe 1 angeben (hier 25\% der Zielangaben, in Gruppe 2 42,42\% und in Gruppe 3 $39,47 \%)$.

\subsubsection{Wahrnehmungs- und Bewältigungskategorien der Mastery-Awareness- Scale als Prädiktoren verschiedener Therapieverläufe}

Folgende Kreisdiagramme vermitteln auch an dieser Stelle einen Überblick der Verteilung zu $\mathrm{t} 1$ angegebener Probleme der drei Erfolgsgruppen auf Wahrnehmungsund Bewältigungskategorien der Mastery-Awareness-Scale. Als 100\% gelten alle Problemangaben der jeweiligen Gruppe zu diesem Zeitpunkt.

Abbildung 31: Verteilung zu t1 angegebener Probleme der drei Erfolgsgruppen auf die Wahrnehmungskategorien der Mastery-Awareness-Scale 
Gruppe 1 (guter Therapieverlauf)

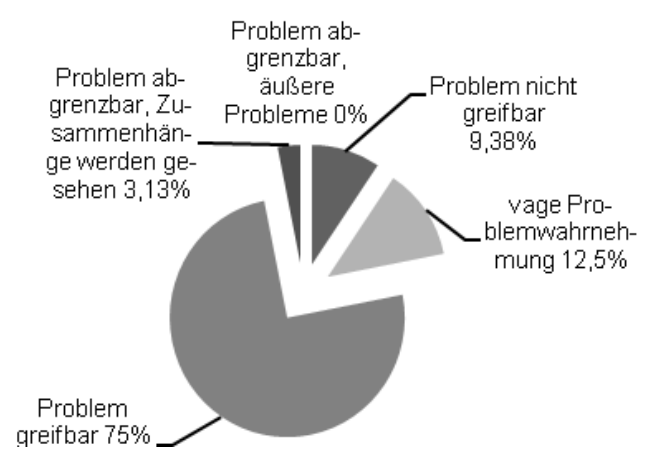

Gruppe 2 (mittlerer Therapieverlauf)

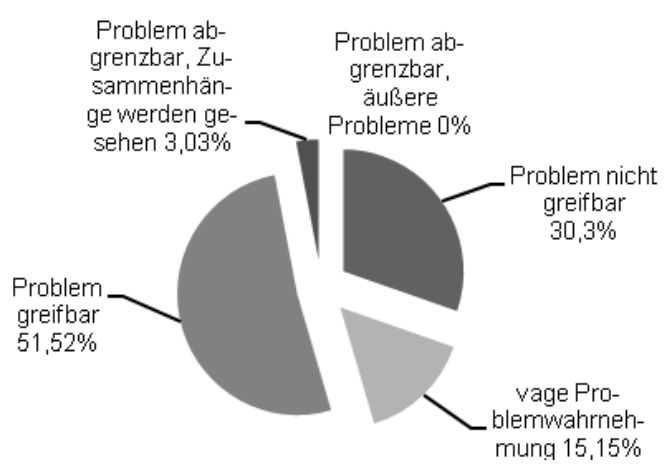

Gruppe 3 (mäßiger Therapieverlauf)

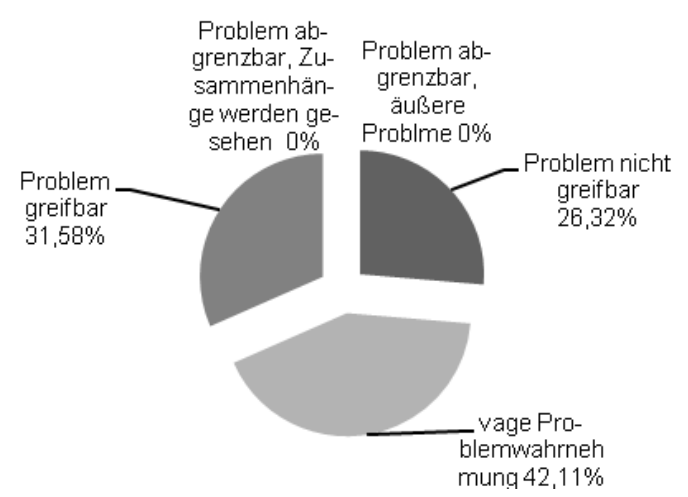

Abbildung 32: Verteilung zu t1 angegebener Probleme der drei Erfolgsgruppen auf Bewältigungskategorien der Mastery-Awareness-Scale

\section{Gruppe 1 (guter Therapieverlauf)}

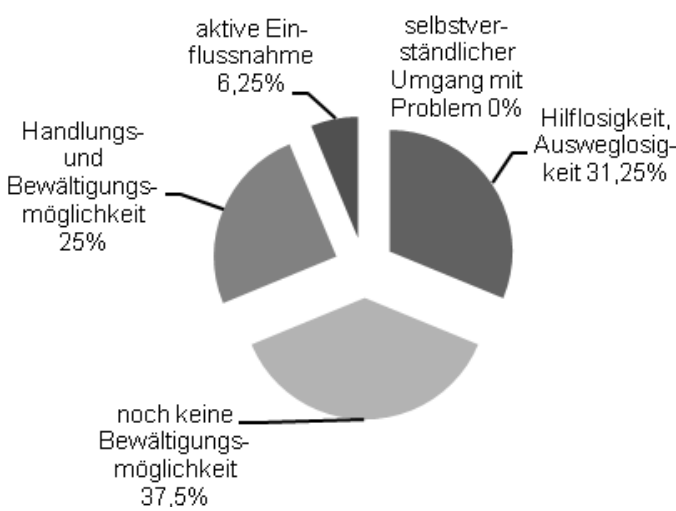

\section{Gruppe 2 (mittlerer Therapieverlauf)}

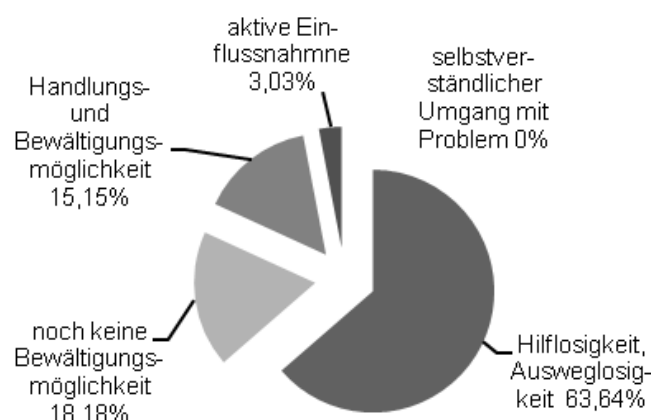




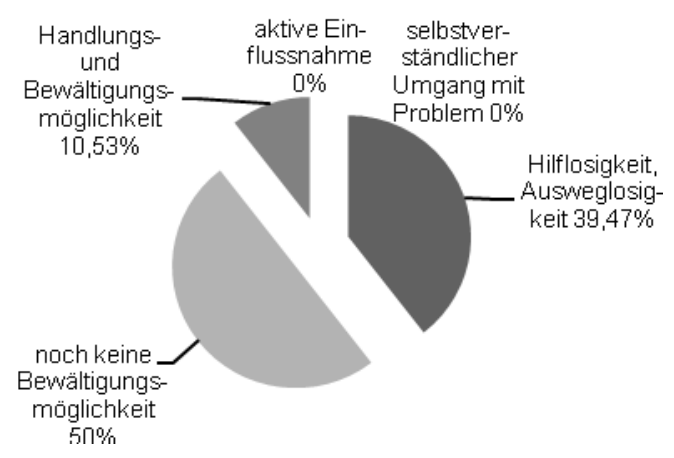

Differenzierte Wahrnehmung und vorhandene Bewältigungsmöglichkeit der Probleme als mögliche Prädiktoren eines guten Therapieverlaufs:

Die Beurteilung der Wahrnehmung der Patienten bezüglich ihrer zu $\mathrm{t} 1$ genannten Probleme (zu diesem Zeitpunkt) mittels Mastery-Awareness-Scale lässt in den drei Erfolgsgruppen erkennen, dass eine "greifbare“ Wahrnehmung dieser Probleme (Kategorie W3) Prädiktor eines guten Therapieergebnisses sein könnte. Demnach beträgt der Anteil derartig erlebter Probleme an allen formulierten Problemen einer Gruppe in Gruppe 1 (guter Therapieverlauf) 75\%, in Gruppe 2 (mittlerer Therapieverlauf) $51,52 \%$ und in Gruppe 3 (mäßiger Therapieverlauf) $31,58 \%$.

Außerdem sind in Erfolgsgruppe 1 zu Erhebungszeitpunkt t1, häufiger als in Gruppe 2 oder 3, „Handlungs- und Bewältigungsmöglichkeiten“ im Umgang mit den angegebenen Problemen (Kategorie M3) vorhanden. Die genauen Prozentsätze betragen $25 \%$ der Problemangaben von Gruppe 1, 15,15\% von Gruppe 2 und 10,53\% der Probleme von Gruppe 3. Diese Kategorie wird demnach als möglicher Prädiktor eines guten Therapieverlaufs in Erwägung gezogen.

Wenig differenzierte Wahrnehmung und geringe Bewältigungsmöglichkeit der Probleme als mögliche Prädiktoren eines mäßigen Therapieverlaufs:

Probleme werden zu t1 vor allem in Gruppe 2 (mittlerer Therapieverlauf) und 3 (mäBiger Therapieverlauf) als "nicht greifbar" erlebt (Kategorie W1): in Gruppe 2 sind $30,3 \%$ der genannten Probleme dieser Kategorie zuzuordnen, $26,32 \%$ sind es in Gruppe 3 und 9,38\% in Gruppe 1. Die vage Problemwahrnehmung (Kategorie W2) ist vornehmlich in Gruppe 3 zu finden (hier werden $42,11 \%$ der Probleme derartig wahrgenommen, in Gruppe 1 werden 12,5\% und in Gruppe $215,15 \%$ der angegebenen Probleme vage beschrieben). 
Werden die Problemangaben der Patienten nach Bewältigungsmöglichkeiten geratet, so zeigt das Ergebnis, dass "Hilflosigkeit und Ausweglosigkeit“ im Umgang mit den angegebenen Problemen (Kategorie M1) zum Erhebungszeitpunkt t1 in Gruppe 2 (mittlerer Therapieerfolg) mit 63,64\% der Probleme dieser Gruppe prozentual häufiger als in Gruppe $1(31,25 \%)$ oder in Gruppe $3(39,47 \%)$ vertreten sind. Damit könnte diese Kategorie Prädiktor für einen mittleren Therapieverlauf sein.

Probleme, bei welchen „noch keine Bewältigungsmöglichkeiten“ vorhanden sind (Kategorie M2), machen zu t1 in Gruppe 3 die Hälfte, in Gruppe $137,5 \%$ und in Gruppe 2 18,18\% der Problemangaben aus. Folglich könnte diese Kategorie Prädiktor eines eher mäßigen Therapieerfolges sein.

Fazit:

Die Ergebnisse der Überprüfung von Hypothese H2.1 wurden im Rahmen dieser Dissertation nicht auf Signifikanz getestet. Die deskriptive Auswertung zeigte jedoch Tendenzen möglicher Prädiktoren für gute und mäßige Therapieverläufe auf. Es muss an dieser Stelle bezüglich der Gruppeneinteilung der hier untersuchten Patienten erneut betont werden, dass es bei allen überprüften Personen zu einer Abnahme (oder selten keiner Veränderung) des Leidensdrucks unter den zu t1 formulierten Problemen kam und folglich nur einschränkend von „mäßigen“ Verläufen berichtet werden kann, da der Leidensdruck niemals anstieg.

\subsection{Prüfung von Hypothese H2.2}

„Die in Hypothese H2.1 ermittelten Prädiktoren für den Erfolg von Therapieverläufen sind innerhalb der beiden untersuchten Therapiegruppen (supportiv-expressive Therapie (SET) und Verhaltenstherapie) unterschiedlich verteilt."

Mit der Prüfung von Hypothese 2.1 wurde untersucht, ob es Hinweise auf Prädiktoren guter oder mäßiger Therapieverläufe gibt. Es zeigte sich dabei, dass zu Erhebungszeitpunkt $\mathrm{t} 1$ bestehende Faktoren wie jüngeres Alter, verhaltenstherapeutische Behandlung, ein hoher Leidensdruck unter zu $\mathrm{t} 1$ angegebenen Problemen zu diesem Zeitpunkt, Angst als Probleminhalt, Angst oder körperliche Beschwerden zu bessern 
als Zielinhalt, Probleme als „greifbar“ wahrzunehmen sowie über „Handlungs- und Bewältigungsmöglichkeiten“ zu verfügen, für einen erfolgreicheren Therapieverlauf im Sinne stärkerer Reduktion des Leidensdrucks unter zu t1 genannten Probleme sprechen könnten. Einen mäßigen Therapieverlauf bedingen möglicherweise zu Befragungszeitpunkt $\mathrm{t} 1$ vorhandene Probleme mit körperlichen Beschwerden, das Ziel „Ruhe und Gelassenheit“ zu finden sowie Probleme als „nicht greifbar“ oder „vage“ zu erleben und für diese „noch keine Bewältigungsmöglichkeit“ zu besitzen.

In der Überprüfung von Hypothese H2.2 wird nun weiter analysiert, welche Merkmale, Probleme oder Ziele in erfolgreichen oder weniger erfolgreichen Untergruppen innerhalb beider nach unterschiedlichen Therapierichtungen (VT versus SET) behandelten Patientengruppen auftreten.

Die Darstellung der Auswertung erfolgt deskriptiv, ein Signifikanztest wurde im Zuge dieser Dissertation nicht durchgeführt. Bei der Bewertung der Ergebnisse muss darüber hinaus berücksichtigt werden, dass die in dieser Hypothesen-Prüfung betrachteten Untergruppen relativ kleine Anzahlen von Patienten aufweisen (acht bis zehn Studienteilnehmer pro Untergruppe).

Um die beiden Psychotherapieformen SET und VT bezüglich möglicher Prädiktoren bestimmter Therapieverläufe getrennt voneinander zu analysieren, wurden die für Hypothese H2.1 überprüften Patienten jeweils innerhalb der Therapierichtung zwei möglichst gleich großen Gruppen (im Folgenden „Untergruppen“) zugeordnet. Diese Einteilung basiert somit auf der Gruppenbildung nach Therapieverlauf zur Überprüfung von Hypothese $\mathrm{H} 2.1$, entscheidend hierbei die Abnahme des Leidensdrucks unter zu t1 formulierten Problemen von Befragungszeitpunkt t1 (3. Therapiesitzung) bis $\mathrm{t} 3$ (Behandlungsende). Wie bereits erwähnt, konnten insgesamt 36 Patienten für die Hypothesen H2.1 und H2.2 überprüft werden, da sie sowohl zu t1, als auch zu t3 Angaben bezüglich des Leidensdrucks machten. Davon wurden 19 verhaltenstherapeutisch und 17 psychodynamisch behandelt.

Zur Überprüfung dieser Hypothese wurde daher folgende Einteilung vorgenommen:

$\underline{\text { VT: }}$

Untergruppe $1=$ guter Therapieverlauf (VT-Patienten aus Gruppe 1 der Prüfung von Hypothese H2.1, zehn Patienten) versus 
Untergruppe $2=$ mittlerer und mäßiger Therapieverlauf (VT-Patienten aus Gruppe 2 und 3 der Prüfung von Hypothese H2.1, neun Patienten)

Psychodynamische Therapie:

Untergruppe $1=$ guter und mittlerer Therapieverlauf (SET-Patienten aus Gruppe 1 und 2 der Prüfung von Hypothese H2.1, acht Patienten) versus

Untergruppe $2=$ mäßiger Therapieverlauf (SET-Patienten aus Gruppe 3 der Prüfung von Hypothese $\mathrm{H} 2.1$, neun Patienten)

Hinsichtlich möglicher Prädiktoren fand innerhalb der 19 für Hypothese H2.1 analysierten, verhaltenstherapeutisch behandelten Studienteilnehmer ein Vergleich zwischen einer Untergruppe von zehn Patienten mit guten, gegen eine Sub-Gruppe von neun Patienten mit mittleren und mäßigen Therapieverläufen statt. Bei den 17 für Hypothese H2.1 untersuchten SET-Patienten bestanden die bezüglich möglicher Prädiktoren untersuchten Sub-Gruppen aus acht Patienten mit gutem und mittlerem Therapieverlauf, gegenübergestellt neun Patienten mit mäßigem Therapieerfolg.

Diese zwischen den beiden Therapieformen unterschiedliche Sub-GruppenEinordnung wurde gewählt, da so eine auf der Erfolgsgruppen-Einteilung von Hypothese $\mathrm{H} 2.1$ basierende, fast gleich große Untergruppen-Zuteilung möglich war. Somit haben innerhalb beider Therapierichtungen annähernd $50 \%$ der Patienten bessere und $50 \%$ schlechtere Verläufe, was wiederum einen eingeschränkten Vergleich zulässt, obwohl die Grenzen der Gruppenzuteilung zwischen beiden Therapieverfahren ungleich sind. Dies ist deshalb unproblematisch, da die Prädiktoren bestimmter Therapieverläufe innerhalb der beiden Therapierichtungen ermittelt werden sollen. Es ist dabei von Interesse, welche Patienten unter der jeweiligen Therapie erfolgreich oder weniger erfolgreich behandelt werden konnten und ob dafür bestimmte Prädiktoren identifiziert werden können.

\subsubsection{Verhaltenstherapie}

Es folgt eine Übersicht der Verteilung aller von den 19 verhaltenstherapeutisch behandelten Patienten zu Befragungszeitpunkt $\mathrm{t} 1$ angegebenen Probleme und Ziele auf Kategorien des Kategoriensystems und der Mastery-Awareness-Scale. Den nachfolgenden Kreisdiagrammen können dabei prozentuale Anteile der einzelnen Kategorien an allen Problem- oder Zielangaben einer Untergruppe zu Befragungszeitpunkt 
t1 entnommen werden. Dabei entsprechen 100\% allen Problem- oder Zielangaben der jeweiligen Untergruppe zu Messzeitpunkt t1.

Abbildung 33: Verteilung der Problem- und Zielangaben von t1 auf Kategorien des Kategoriensystems,

\section{Erfolgsuntergruppen verhaltenstherapeutisch behandelter Patienten}

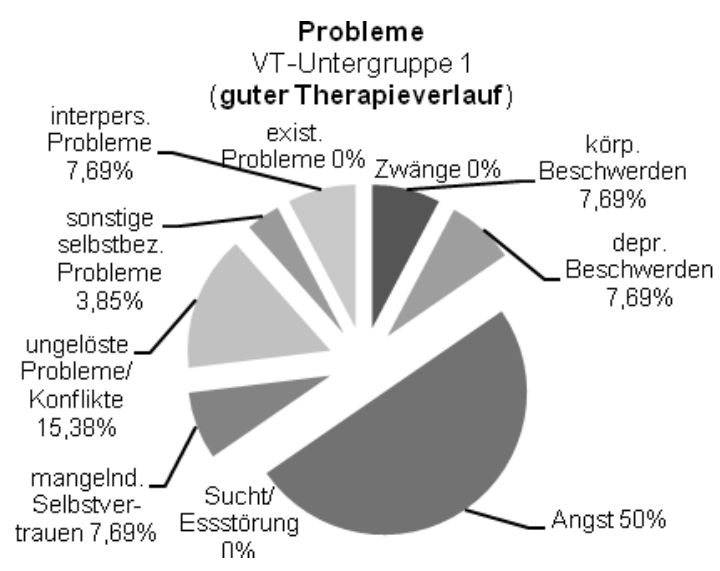

Ziele

VT-Untergruppe 1 (guter Therapieverlauf) körp

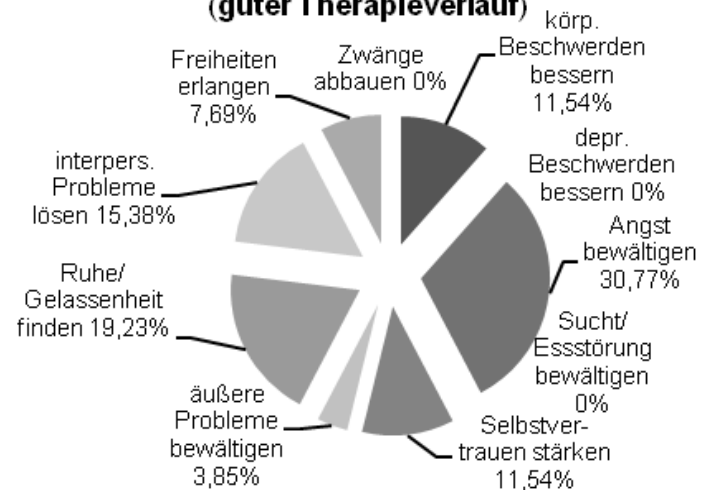

Probleme

VT-Untergruppe 2

(mittlerer und mäßiger Therapieverlauf)

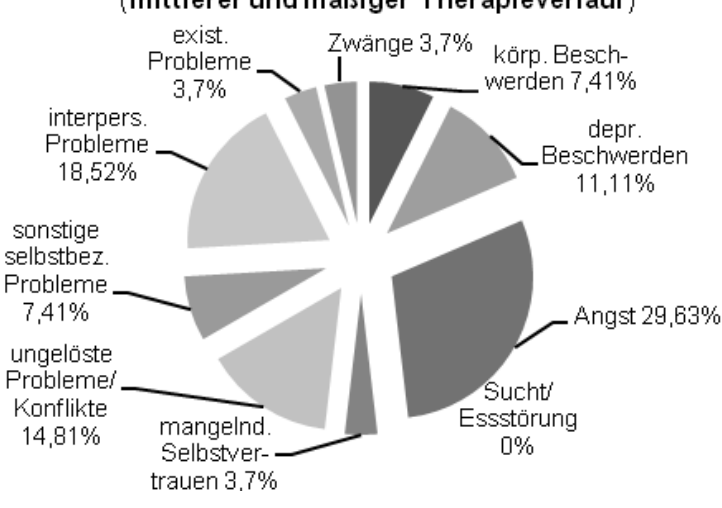

Ziele

VT-Untergruppe 2 (mittlerer und mäßiger Therapieverlauf)

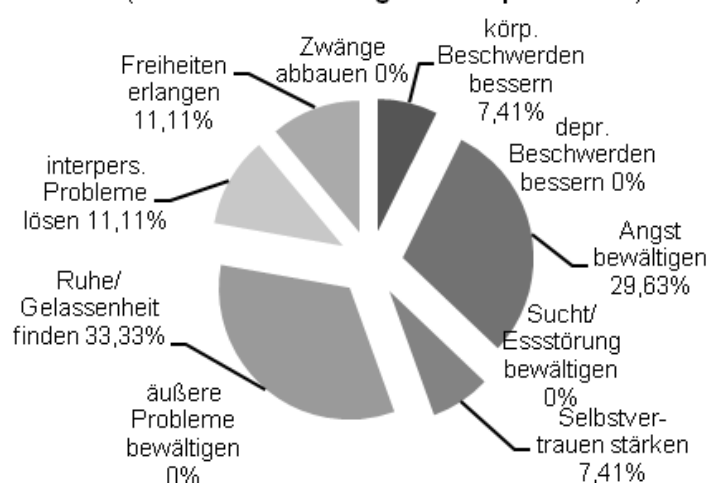

Abbildung 34: Verteilung der Problemangaben von t1 auf Kategorien der MasteryAwareness-Scale, Erfolgsuntergruppen verhaltenstherapeutisch behandelter Patienten
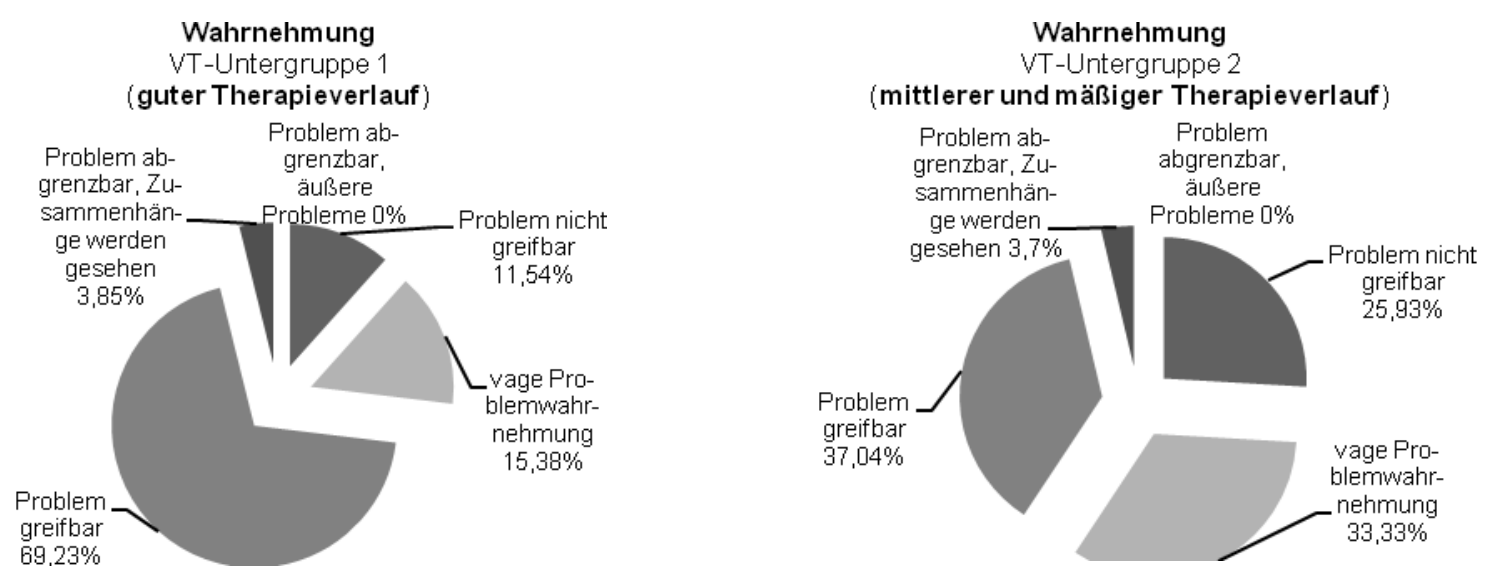

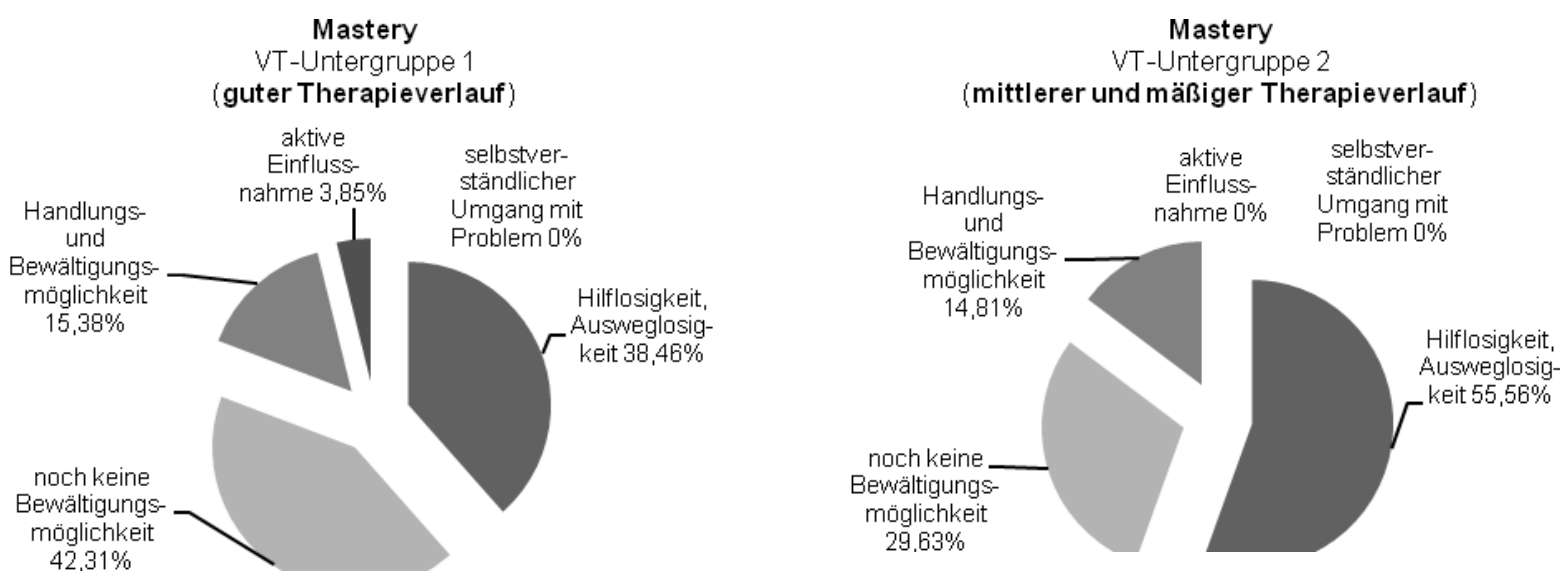

Mögliche Prädiktoren eines erfolgreichen Therapieverlaufs verhaltenstherapeutisch behandelter Patienten (VT-Untergruppe 1):

Verhaltenstherapeutisch behandelte Studienteilnehmer der erfolgreicheren Untergruppe 1 haben im Mittel ein jüngeres Alter als diejenigen der weniger erfolgreichen VT-Untergruppe 2. Demnach liegt in Untergruppe 1 das Durchschnittsalter zu Behandlungsbeginn bei 41 Jahren (arithmetisches Mittel, SD = 12), während es in Untergruppe 2 bei 51 Jahren liegt (arithmetisches Mittel, SD = 10).

Darüber hinaus ist der Median des Leidensdrucks unter den zu Befragungszeitpunkt t1 angegebenen Problemen in Untergruppe 1 höher (4 = Leidensdruck könnte nicht schlimmer sein) als in Sub-Gruppe 2 (hier beträgt der Median 3 = starker Leidensdruck).

Die inhaltliche Analyse der Angaben des Befragungszeitpunktes $\mathrm{t} 1$ mittels des Kategoriensystems zeigt, dass Probleme des Inhaltes Angst in der erfolgreichen Untergruppe 1 der VT-Patienten häufiger genannt werden (die Hälfte aller Problemangaben dieser Gruppe), als in Untergruppe 2 mit mäßigem Therapieverlauf (hier knapp ein Drittel Problemnennungen).

Die Beurteilung der Problemangaben mit der Mastery-Awareness-Scale ergibt bezüglich der Problemwahrnehmung, dass die meisten Probleme in Untergruppe 1 als "greifbar" erlebt werden (69,23\% der zu t1 genannten Probleme dieser Gruppe), was in Untergruppe 2 deutlich seltener der Fall ist (37,04\%). Das Rating der Angaben nach der Problembewältigung ergibt, dass in Untergruppe 1 häufiger „noch keine Bewältigungsmöglichkeit“ vorhanden ist (42,31\% Kategorie B2, welche jedoch aussagt, mehr Bewältigungsmöglichkeiten zur Verfügung zu haben als in Kategorie B1/ „Hilflosigkeit“), während diese Kategorie in der Sub-Gruppe 2 seltener auftritt $(29,63 \%)$. 
Mögliche Prädiktoren eines weniger erfolgreichen Therapieverlaufs verhaltenstherapeutisch behandelter Patienten (VT-Untergruppe 2):

Die Auswertung mit dem Kategoriensystem zeigt, dass interpersonelle Probleme und depressive Beschwerden öfters in Untergruppe 2 vorkommen. Das Ziel, Ruhe und Gelassenheit zu finden, wird zu $\mathrm{t} 1$ in Untergruppe 2 ebenfalls häufiger angegeben (ein Drittel der Problemäußerungen), als in der erfolgreichen VT-Untergruppe 1 (19,23\% der Probleme).

Aus dem Rating mit der Mastery-Awareness-Scale bezüglich der Wahrnehmung resultiert zudem, dass Probleme in Gruppe 2 öfters als "nicht greifbar" wahrgenommen werden (rund ein Viertel der Angaben, während in der erfolgreicheren SubGruppe 1 nur 11,54\% der Problemangaben dieser Kategorie zuzuordnen sind). AuBerdem wird eine vage Problemwahrnehmung (Kategorie W2) in Untergruppe 2 häufiger beschrieben (ein Drittel der Probleme) als in der Untergruppe mit gutem Therapieverlauf (hier 15,38\%). Darüber hinaus ergibt die Untersuchung der Bewältigungsmöglichkeiten der aufgezählten Probleme, dass über die Hälfte der Problemaussagen von Untergruppe 2 „Hilflosigkeit und Ausweglosigkeit“ im Umgang mit dem Problem erkennen lassen (zum Vergleich: in der guten VT-Gruppe nur 38,46\%).

\subsubsection{Psychodynamische Therapie (SET)}

Es folgen Kreisdiagramme für eine Übersicht der Aufteilung der Patientenangaben auf die Kategorien des Kategoriensystems und der Mastery-Awareness-Scale. Dabei entsprechen $100 \%$ jeweils allen Problemen bzw. Zielen, die zu Befragungszeitpunkt t1 von den für diese Hypothese ausgewerteten SET-Patienten einer Untergruppe genannt wurden. 
Abbildung 35: Verteilung der Problem- und Zielangaben von t1 auf Kategorien des Kategoriensystems,

Erfolgsuntergruppen psychodynamisch behandelter Patienten
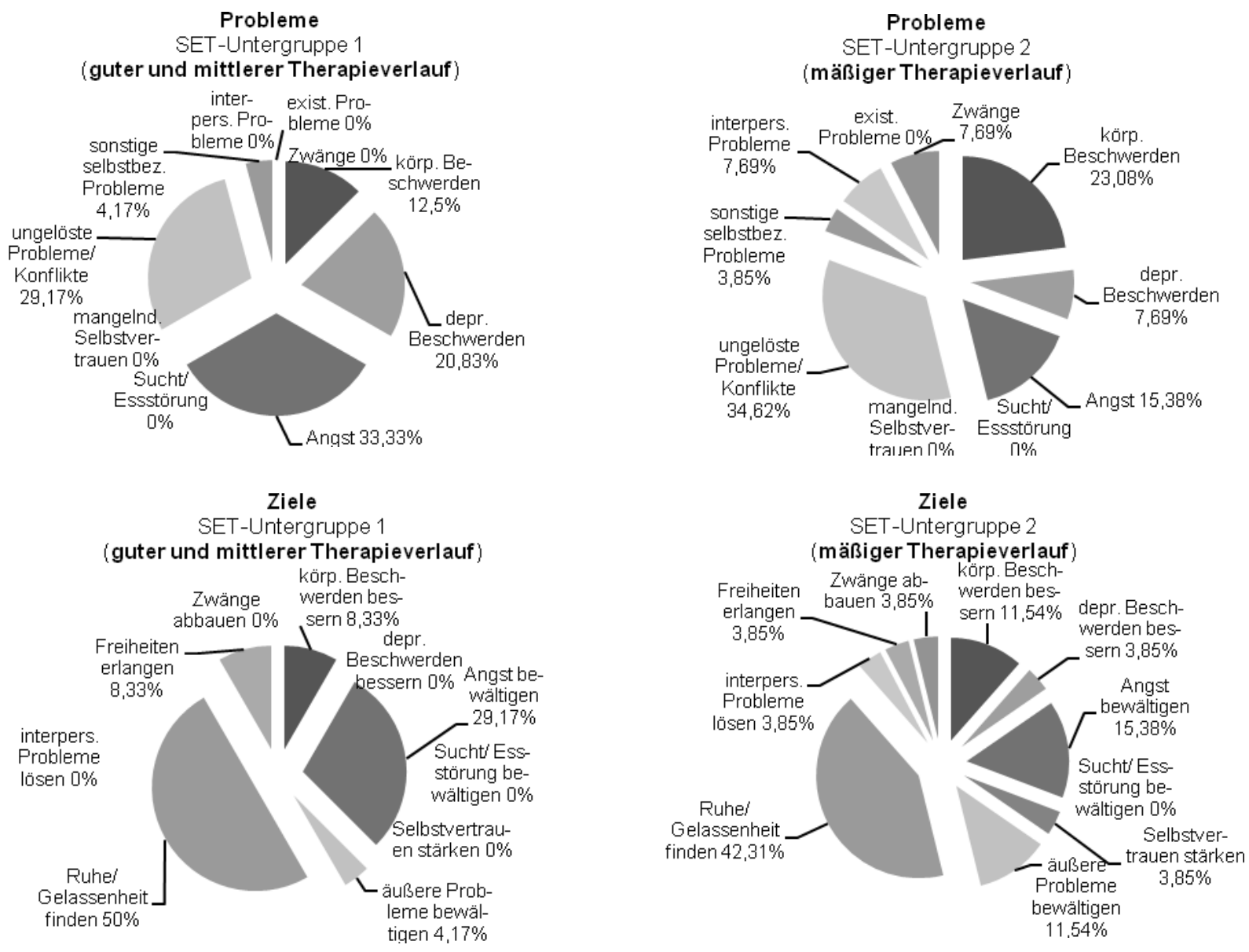

Abbildung 36: Verteilung der Problemangaben von t1 auf Kategorien der Mastery-

Awareness-Scale, Erfolgsuntergruppen psychodynamisch behandelter Patienten
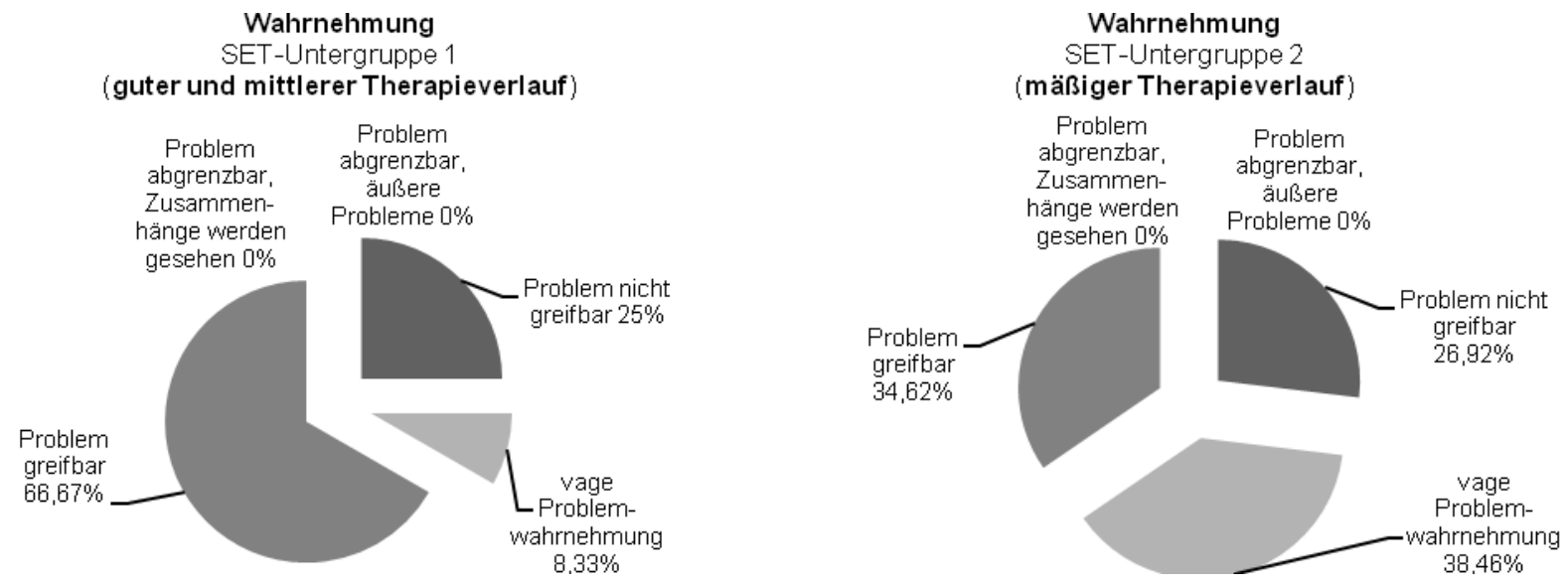

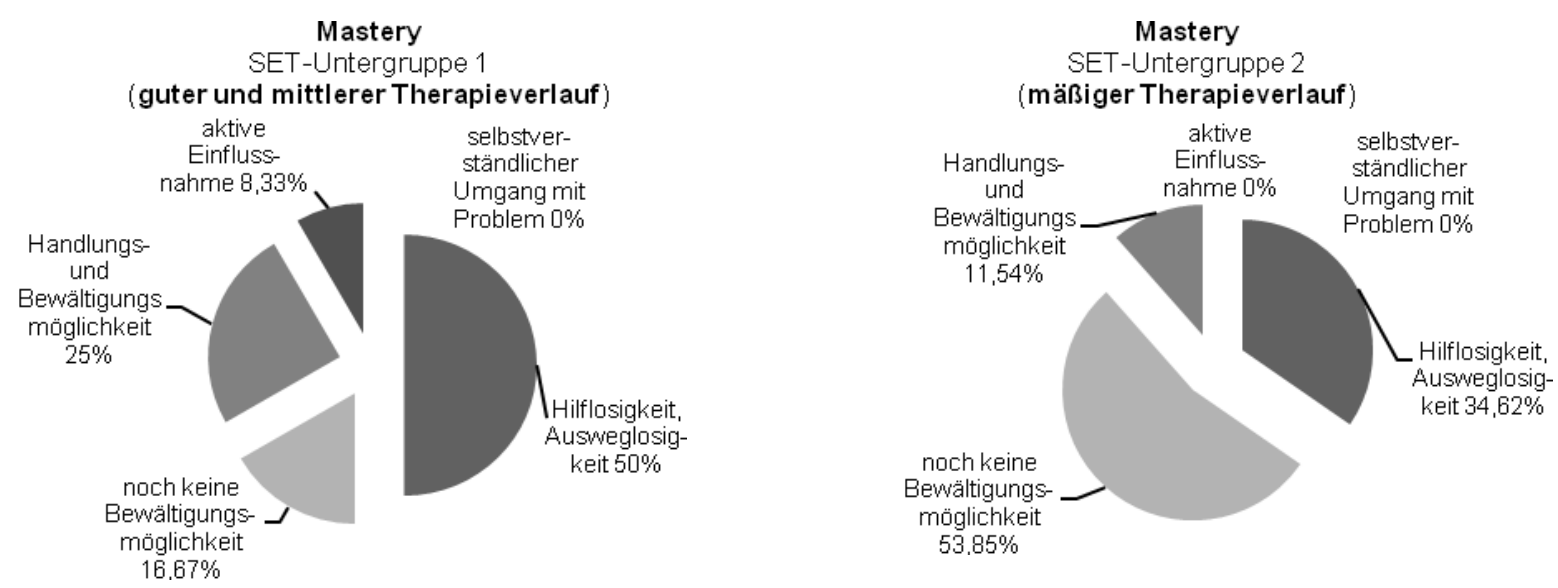

Mögliche Prädiktoren eines erfolgreichen Therapieverlaufs psychodynamisch behandelter Patienten (SET-Untergruppe 1):

Patienten der erfolgreicheren SET-Sub-Gruppe 1 sind im Mittel jüngeren Alters als die der Untergruppe 2 mit mäßigem Therapieverlauf: Das Alter zu Therapiebeginn liegt in Untergruppe 1 bei 34 Jahren (arithmetisches Mittel, $S D=6$ ), während es in Untergruppe 2 bei 46 Jahren liegt (arithmetisches Mittel, SD =11).

Geratet nach dem Kategoriensystem kommen inhaltlich in der SET-Untergruppe 1 zum Zeitpunkt t1 öfter depressive Beschwerden vor als in Untergruppe 2. Darüber hinaus beinhalten in Untergruppe 1 ein Drittel der Probleme Angst, während es in Untergruppe 2 nur 15,38\% der Angaben sind. Dementsprechend enthalten die Zielangaben von Sub-Gruppe 1 zu t1 häufiger das Ziel, Angst zu bewältigen, als dies in der Vergleichsgruppe 2 der Fall ist.

Das Rating der Problemäußerungen von 11 mit der Mastery-Awareness-Scale zeigt, dass bezüglich der Problemwahrnehmung in der überlegenen Sub-Gruppe 1 zwei Drittel der Probleme als greifbar erlebt werden, während dies in Untergruppe 2 seltener vorkommt (rund ein Drittel der Probleme). Das Rating der Problembewältigung aller verglichenen SET-Patienten ergibt, dass zu t1 „Hilflosigkeit/ Ausweglosigkeit“ im Umgang mit dem Problem (Kategorie B1) in Untergruppe 1 häufiger auftritt (die Hälfte der Problemangaben, in Sub-Gruppe $2 \mathrm{ca}$. ein Drittel der Problemnennungen). Ebenfalls öfters ist hier Kategorie „Handlungs- und Bewältigungsmöglichkeit im Umgang mit dem Problem" (B3) vorhanden (ein Viertel der genannten Probleme von Untergruppe 1, während es in Untergruppe $211,54 \%$ sind). Häufiger in Sub-Gruppe 1 , aber insgesamt relativ selten werden Probleme als „aktiv beeinflussbar“ (Kategorie 4a) erlebt. 
Mögliche Prädiktoren eines weniger erfolgreichen Therapieverlaufs psychodynamisch behandelter Patienten (SET-Untergruppe 2):

Die inhaltliche Prüfung der Problemaussagen mit Hilfe des Kategoriensystems zeigt, dass zu Befragungszeitpunkt $\mathrm{t} 1$ unter den SET-Patienten solche mit einem eher mäßigen Therapieverlauf (Untergruppe 2) häufiger körperliche Beschwerden nennen, als diejenigen mit erfolgreichem Therapieverlauf (Untergruppe 1).

Bezüglich der Problemwahrnehmung ergibt die Überprüfung mit der MasteryAwareness-Scale, dass zu t1 in Sub-Gruppe 2 häufiger (38,46\%) Probleme als vage wahrgenommen wurden, als in der besseren Untergruppe 1 (hier 8,33\%). Die Bewältigungsmöglichkeiten zu 11 genannter Probleme zu diesem Zeitpunkt wurden ebenfalls mittels der Mastery-Awareness-Scale näher betrachtet. In Untergruppe 2 lässt über die Hälfte der angegeben Probleme „noch keine Bewältigungsmöglichkeit“ (Kategorie B2) erkennen, in der besseren SET-Untergruppe 1 hingegen werden 16,67\% der Problemnennungen dieser Kategorie zugeordnet.

\subsubsection{Vergleich der Verteilung von Prädiktoren innerhalb der Therapierichtun- gen VT und SET}

Wegen der unterschiedlichen Sub-Gruppen-Einteilung der Studienteilnehmer ist ein Vergleich hinsichtlich möglicher Prädiktoren bestimmter Therapieverläufe zwischen den Behandlungsrichtungen nur eingeschränkt aussagekräftig. Für die Prüfung von Hypothese H2.2 gehört die mittlere Erfolgsgruppe aus Hypothese H2.1 bei verhaltenstherapeutisch behandelten Patienten zu Untergruppe 2, bei psychodynamisch therapierten Patienten zu Untergruppe 1. Ein Vergleich unter Vorbehalt der Berücksichtigung oben genannter Problematik zeigt jedoch folgende Differenzen (und z.T. Übereinstimmungen) der Verteilung möglicher Prädiktoren verschiedener Therapieverläufe zwischen den beiden psychotherapeutischen Verfahren auf:

In den erfolgreicheren Untergruppen 1 wird bei den verhaltenstherapeutisch behandelten Patienten zu t1 (3. Therapiesitzung) ein höherer Leidensdruck unter zu diesem Zeitpunkt angegebenen Problemen als in VT-Untergruppe 2 gefunden. Außerdem sehen erfolgreiche VT-Patienten hier häufig "noch keine Bewältigungsmöglichkeit" genannter Probleme, was bei den erfolgreichen psychodynamisch therapierten Studienteilnehmern nicht der Fall ist. In der erfolgreichen SET-Untergruppe 1 zeigen sich bei vielen Patienten depressive Beschwerden und „Hilflosigkeit/ Ausweglo- 
sigkeit" im Umgang mit Problemen, was bei VT-Patienten vielmehr für weniger erfolgreiche Therapieverläufe sprechen könnte, da diese eher in Sub-Gruppe 2 vorkommen. Darüber hinaus beschreiben erfolgreich psychodynamisch behandelte Studienteilnehmer zu $\mathrm{t} 1$ öfters „Handlungs- und Bewältigungsmöglichkeiten“ der Probleme, als dies in der SET-Untergruppe 2 der Fall ist. Selten, aber dennoch vorkommend werden Probleme sogar als „aktiv beeinflussbar" erlebt.

Den erfolgreichen Untergruppen 1 beider Therapierichtungen sind das jüngere Alter im Vergleich zu den weniger erfolgreichen Untergruppen 2, Probleme des Inhaltes Angst und die als "greifbar" beschriebene Wahrnehmung der Probleme zum Erhebungszeitpunkt $\mathrm{t} 1$ gemeinsam.

In der weniger erfolgreichen Untergruppe 2 der VT-Patienten werden zu t1 häufiger als in der besseren VT-Untergruppe 1 „depressive Beschwerden“ (bei SET eher in der guten Untergruppe 1) und "interpersonelle Probleme“ angegeben. Außerdem werden öfters Ziele des Inhaltes „Ruhe oder Gelassenheit“ zu finden, formuliert. Probleme des Zeitpunktes $\mathrm{t} 1$ werden oft als "nicht greifbar" beschrieben und es herrscht „Hilflosigkeit/ Ausweglosigkeit“ im Umgang mit innen. Letztere Kategorie ist, wie bereits erläutert, bei den psychodynamisch therapierten Studienteilnehmern eher in der erfolgreicheren Untergruppe $1 \mathrm{zu}$ finden.

In der SET-Untergruppe 2 mit mäßigem Therapieerfolg stehen zu t1 körperliche Beschwerden stärker im Vordergrund. Bezüglich der Problembewältigung herrscht öfters als in der besseren SET-Erfolgsgruppe 1 „noch keine Bewältigungsmöglichkeit“. Diese Kategorie ist bei den verhaltenstherapeutisch betreuten Patienten eher in der besseren Untergruppe 1 vertreten.

Ähnlichkeiten bestehen zwischen beiden Untergruppen 2 in Bezug auf die zum häufig „vage Problemwahrnehmung“ angegebener Probleme Zeitpunkt t1.

\section{Fazit:}

Aufgrund methodischer Einschränkungen kann die vorliegende Hypothese in ihrer Gültigkeit nicht eindeutig bestätigt oder abgelehnt werden, sie wurde daher innerhalb dieser Dissertation nicht bezüglich der Signifikanz ihrer Ergebnisse untersucht. Es ergeben sich jedoch durch die deskriptiv dargestellten, vorbehaltlichen Vergleiche der Erfolgsgruppen beider Therapierichtungen 
Hinweise darauf, dass je nach Psychotherapieverfahren Unterschiede betreffend potentieller Prädiktoren guter oder weniger erfolgreicher Therapieverläufe bestehen. Beispielweise werden depressive Beschwerden zu Therapiebeginn (t1) bei VT eher in der weniger erfolgreichen und bei SET in der erfolgreichen Gruppe angegeben.

\subsection{Motivation zur Studienteilnahme}

Insgesamt sank mit fortschreitender Studiendauer die Bereitschaft der Patienten, PATH-Fragebögen vollständig auszufüllen. So wurden zum Ende der Therapie häufiger keine Angaben mehr gemacht. Zu t1 liegt der Prozentsatz der fehlenden an allen möglichen 153 Angaben bei den Problemen bei 3,92\% und bei den Zielen bei 3,27\%. Zu t5 liegt dieser Prozentsatz bei den Problemen bei 39,22\% und bei den Zielen bei $38,56 \%$. Allerdings sind in diesen Zahlen auch drei Patienten enthalten, die im Katamnese-Jahr (also nach Therapieende) wegen zusätzlicher Behandlungsbedürftigkeit oder somatischer Erkrankung nicht mehr an der Studie teilnahmen.

Dies verdeutlicht, dass zu den unterschiedlichen Erhebungszeitpunkten $\mathrm{t} 1$ bis $\mathrm{t} 5$ nicht immer die gleiche Anzahl von Antworten vorlag. Um diesem Problem Rechnung zu tragen, wurde zu den Angaben der absoluten Häufigkeit stets die Angabe des Prozentsatzes der Nennungen einer Kategorie an zum jeweiligen Zeitpunkt vorhandenen Problem- oder Zielaussagen gewählt.

Tabelle 23: zeitlicher Verlauf von fehlenden Problem- oder Zielangaben

\begin{tabular}{|c|c|c|c|c|c|}
\hline \multicolumn{3}{|c|}{ fehlende Problemangaben } & \multicolumn{3}{|c|}{ fehlende Zielangaben } \\
\hline & $\begin{array}{l}\text { absolute } \\
\text { Häufigkeit }\end{array}$ & $\begin{array}{c}\text { \%-Anteil an möglichen } \\
153 \text { Problemangaben } \\
\text { der Patienten zum } \\
\text { jeweiligen Zeitpunkt }\end{array}$ & & $\begin{array}{l}\text { absolute } \\
\text { Häufigkeit }\end{array}$ & $\begin{array}{c}\text { \%-Anteil an möglichen } \\
153 \text { Zielangaben } \\
\text { der Patienten zum } \\
\text { jeweiligen Zeitpunkt }\end{array}$ \\
\hline t1 & 6 & $3,92 \%$ & t1 & 5 & $3,27 \%$ \\
\hline t2 & 18 & $11,76 \%$ & t2 & 19 & $12,42 \%$ \\
\hline t3 & 39 & $25,49 \%$ & t3 & 35 & $22,88 \%$ \\
\hline t4 & 24 & $15,69 \%$ & t4 & 22 & $14,38 \%$ \\
\hline t5 & 60 & $39,22 \%$ & t5 & 58 & $38,56 \%$ \\
\hline
\end{tabular}


Außer der Kategorie „keine Angaben" wurde noch eine andere Art von Aussage, welche keiner Inhaltskategorie zugeordnet werden konnte, berücksichtigt: Formulierungen wie „derzeit kein Problem“, „aktuell keine Ziele“, etc. wurden nicht in die Gruppe ohne Angaben subsummiert, sondern separat als Kategorie gezählt und ausgewertet. Ein Anstieg dieser Kategorie ergibt sich gleichermaßen mit fortschreitendem Therapieverlauf.

Tabelle 24: zeitlicher Verlauf von Angaben, kein Problem oder Ziel zu haben

\begin{tabular}{|c|c|c|c|c|c|}
\hline \multicolumn{3}{|c|}{ „kein Problem“ und ähnliche Angaben } & \multicolumn{3}{|c|}{ „kein Ziel“ und ähnliche Angaben } \\
\hline & $\begin{array}{l}\text { absolute } \\
\text { Häufigkeit }\end{array}$ & $\begin{array}{c}\text { \%-Anteil an möglichen } \\
153 \text { Problemangaben } \\
\text { der Patienten zum } \\
\text { jeweiligen Zeitpunkt }\end{array}$ & & $\begin{array}{l}\text { absolute } \\
\text { Häufigkeit }\end{array}$ & $\begin{array}{c}\text { \%-Anteil an möglichen } \\
153 \text { Zielangaben } \\
\text { der Patienten zum } \\
\text { jeweiligen Zeitpunkt }\end{array}$ \\
\hline t1 & 0 & $0 \%$ & t1 & 0 & $0 \%$ \\
\hline t2 & 6 & $4,44 \%$ & t2 & 6 & $4,48 \%$ \\
\hline t3 & 3 & $2,63 \%$ & t3 & 9 & $7,63 \%$ \\
\hline t4 & 9 & $6,98 \%$ & t4 & 13 & $9,92 \%$ \\
\hline t5 & 0 & $0 \%$ & t5 & 6 & $6,32 \%$ \\
\hline
\end{tabular}




\section{Diskussion}

\subsection{Positive Aspekte/ Stärken}

Studiendesign:

Der zentrale Ansatzpunkt der vorliegenden Studie besteht in der Untersuchung des Prozessverlaufs unter naturalistischen Bedingungen, was einen hohen Praxisbezug herstellt. Vorteile liegen darin, dass sie nicht schulenspezisfisch durchgeführt wurde, da die Konzeption von verhaltenstherapeutisch und psychodynamisch orientierten Forschern erfolgte. Dabei wurden Kriterien einer randomisierten, kontrollierten Studie erfüllt sowie mehrere Instrumente zur Datenerhebung vereint (u. A. PATH, was die mit dieser Arbeit ausgewerteten Daten lieferte).

\section{Evaluationsinstrument PATH:}

In der Anwendung dieses Instrumentes geht es um die Erfassung von Problemen und Zielen, welche die Patienten selbst benennen und hinsichtlich des Leidensdrucks und der Zielverwirklichung einschätzen. Diese Angaben beziehen sich nicht nur auf das unmittelbare Krankheitsgeschehen und die damit verbundenen Therapieziele. Die Bedeutung solcher störungsübergreifender Therapieziele wird auch von Trachsel et al. (2008) betont, die in Bezug auf eine verhaltenstherapeutische Studie phobischer Angststörungen vorschlagen, „individuelle Therapieziele zu vereinbaren“ (Trachsel et al. 2008, S. 364).

Mit PATH werden die Probleme und Ziele durch offene Fragestellung ohne Einschränkungen wiederholt erhoben. Fragebögen mit vorgegebenen Antwortmöglichkeiten haben demgegenüber den Nachteil, dass sie die Befindlichkeit der Patienten oft nicht gut genug erfassen können. Viele Instrumente zur Auswertung frei formulierter Angaben arbeiten mit häufig zu eng eingegrenzten Kategorien, denen sich nicht zwangsläufig alle dieser Aussagen zuordnen lassen.

Auch Grawe hebt die Bedeutung der Selbstbeurteilung durch Patienten hervor. „Das einfachste, aber auch relevanteste Kriterium dafür, ob eine Therapie das bewirkt, was sie bewirken soll, ist ja, den Patienten direkt zu fragen, ob und in welcher Hinsicht inm die Therapie genützt hat." (Grawe et al. 1994, S. 170). Die Bedeutung der Selbsteinschätzung hinsichtlich positiver Ergebnisse von Therapien bestätigen auch Fitzpatrick et al. (2005) in einer Studie zur Zusammenarbeit zwischen Patient und Therapeut (therapeutische Arbeitsbeziehung). Somit wird der Selbsteinschätzung der 
Patienten ein zentraler Stellenwert hinsichtlich der Aussagekraft im Vergleich zur Fremdeinschätzung (z.B. durch den Therapeuten) eingeräumt.

PATH unterscheidet sich von vergleichbaren Instrumenten, wie dem Personal Questionnaire (Elliot et al. 1999) oder der Goal Attainment Scaling (Kiresuk und Sherman 1968), dadurch, dass die Angaben der Patienten nicht modifiziert oder als Fokussierung durch den Auswertenden umformuliert und nicht nur auf anfänglichen Aussagen der Patienten basieren (Frölich 2009). In ihrem Ziel der Erfassung auch struktureller Veränderungen im Psychotherapieprozess sind zudem Instrumente wie die Operationalisierte Psychodynamische Diagnostik (OPD, Grande et al. 2005) und die Heidelberger Umstrukturierungsskala (HSCS, Rudolf et al. 2000) ähnlich ausgerichtet wie PATH. Sie sind jedoch eher an Diagnosen und der Symptomatik orientiert. Für die Beurteilung von Patienten-Aussagen mit diesen Instrumenten sind außerdem längere Interviews nötig, daher sind sie in klinischer Routine nur eingeschränkt verwendbar. Die schriftlichen Selbsteinschätzungen der Patienten durch PATH lassen sich in der ambulanten Praxis einfacher durchführen.

Der Vorteil, innerhalb eines Patientenkollektives mit der gleichen psychischen Störung (hier GAS) Probleme, Ziele und Leidensdruck im Verlauf zu beurteilen, liegt darin, dass sie näher am Krankheitsempfinden der Patienten liegen, als allein die Beurteilung über die Diagnose zulässt. So sind die Ziele und Probleme bei Patienten mit GAS durchaus unterschiedlich und verlangen damit nach einer diesen Voraussetzungen angepassten Psychotherapie.

Die alleinige Diagnose (wie z.B. mittels Klassifikation des ICD-10 ${ }^{10}$ kodiert) ist laut Freyberger und Schneider (2001) nur begrenzt verwendbar, wenn es darum geht, Indikationen besonderer Behandlungen herzuleiten und darüber hinaus die Prognose eines Krankheitsbildes zu liefern, oder gar die Wirksamkeit von Behandlungsverfahren zu überprüfen. Hohage (2001) stellt fest, dass gerade im Bereich psychischer Störungen nicht immer objektive Fakten, welche durch bestimmte Therapien voraussehbar modifizierbar behandelt werden können, vorliegen. Desweiteren berücksichtigt die Konzentration auf Funktionseinschränkungen, wie sie in der ICD Klassifikation stattfindet, z.B. nicht den Leidensdruck der einzelnen Patienten. Daher sind Indikati-

\footnotetext{
${ }^{10}$ ICD $10=$ Internationale statistische Klassifikation der Krankheiten und verwandter Gesundheitsprobleme, 10. Revision (DIMDI 2008)
} 
onen zur psychotherapeutischen Behandlung nur basierend auf derartigen Funktionsausfällen, ohne den Leidensdruck mit einzubeziehen, nicht praxisnah.

PATH kann unterschiedlichen therapeutischen Verfahren in Kurz- oder Langzeittherapie gerecht werden, da auch neu hinzukommende Probleme und Ziele im Therapieverlauf erfasst werden können. Staats merkt hierzu an: „Auch neu aufgetretene Probleme werden in Psychoanalysen (...) üblicherweise bearbeitet. Dies wird als ein Grund dafür angesehen, das Psychoanalysen länger dauern können als Verhaltenstherapien: sie beschränken sich nicht auf vor der Therapie definierte Probleme. Die Effektivität analytischer Therapie kann daher nicht erfasst werden, wenn nur die Veränderung vorher definierter Probleme untersucht wird." (Staats 2000, S. 23).

Zukünftig bestünde die Möglichkeit, persönliche Merkmale der Patienten (Prädiktoren, siehe dazu Hypothese H2.2 unter 3.8) bei der Indikationsstellung für eine bestimmte psychotherapeutische Behandlung (VT oder psychodynamische Therapie) zu berücksichtigen.

In der bisherigen Verwendung des Evaluationsinstrumentes PATH wurde die Rückmeldung der Patienten in Form ausgefüllter PATH-Fragebögen von dem behandelnden Psychotherapeuten getrennt ausgewertet. Dieser hatte somit keinen Einblick in angegebene Probleme und Ziele sowie die Selbsteinschätzung der Patienten. Die Anwendung von PATH könnte zukünftig erweitert werden, indem das Verfahren als klinische Feedback-Methode fungiert. Dies würde bedeuten, dass behandelnde Therapeuten Einsicht in die ausgefüllten Fragebögen erhalten oder diese auswerten. „Im Laufe der Therapie können die Ziele dazu verwendet werden, den Fortschritt der Therapie zu prüfen." (Leichsenring et al. 2005, S. 263). Gleichzeitig könnte so der Frage nachgegangen werden, inwieweit Beurteilungen von Patienten bezüglich des Therapieverlaufs denen der behandelnden Psychotherapeuten entsprechen.

\subsection{Einschränkungen der Untersuchungen}

Eine Einschränkung bezüglich der Beurteilung der Ergebnisse insgesamt stellt der Stichprobenumfang von $\mathrm{n}=51$ Patienten dar. Da vor allem mittels des Kategoriensystems die Problem- und Zielaussagen vielen Unterkategorien zugeordnet werden, ist die Anzahl der Nennungen in einigen dieser Kategorien recht klein, was die Aussagekraft der Ergebnisse schmälern kann. 
Weiterhin einschränkend berücksichtigt der Vergleich von manualisierter VT und manualisierter, psychodynamischer Kurzzeittherapie laut Milrod (2009) nicht, dass es im Spektrum der psychodynamischen Verfahren auch andere Orientierungen als die SET-Methode gibt. Der Preis der SET-Manualisierung läge darin, dass die Flexibilität anderer Arten psychodynamischer Psychotherapie vernachlässigt würde. Insofern sollte ein Vergleich, welcher den Anspruch hat, psychodynamische Therapie VT gegenüberzustellen, unter dieser Einschränkung betrachtet werden. Dieses Argument könnte auch bezüglich des in der Studie benutzen Manuals der VT bei GAS (Leibing et al. 2003) gelten, da auch hier eine bestimmte Form der Behandlung stellvertretend für das ganze Spektrum der VT steht.

Zudem sieht Milrod (2009) methodische Probleme im Studiendesign der mit der vorliegenden Dissertation ausgewerteten Studie. Wünschenswert für zukünftige Studien sei eine deutlich höhere Zahl an Studienteilnehmern

Diskutiert werden sollte ebenso die Frage, inwiefern eine eindeutige und sinnvolle Zuordnung der Problem- und Zielaussagen zu den einzelnen Kategorien des Kategoriensystems und der Mastery-Awareness-Scale möglich ist. Das Rating der Patienten-Aussagen wurde durch zwei Rater vorgenommen, die nicht an der Entwicklung der eingesetzten Skalen beteiligt waren. Diesem Problem wurde jedoch begegnet, indem sogenannte Ankerbeispiele zu den einzelnen Kategorien der Skalen vorlagen, sowie die Interrater-Reliabilität mit Cohens Kappa (Bortz 2005) überprüft wurde. Die Ergebnisse lagen dabei für die Kodierung der Patientenangaben mit dem Kategoriensystem im sehr guten Bereich (Kappa Problem-Rating = 0,7, Kappa Ziel-Rating = $0,75)$ und für die Kodierung mit der Mastery-Awareness-Scale im befriedigenden Bereich (Kappa Wahrnehmungs-Rating =0,43, Kappa Bewältigungs-Rating $=0,47$ ).

Eine Weiterentwicklung der Mastery-Awareness-Scale könnte die Anwendung auch auf Zielaussagen der Patienten beinhalten. Damit wäre ein umfassenderer Vergleich mit dem Kategoriensystem, welches ein Rating von Problemen und Zielen ermöglicht, durchführbar.

\section{Signifikanz-Prüfung}


Bei der bedeutenden Frage nach der Entwicklung des Leidensdrucks und der Zielverwirklichung der Patienten wurden Signifikanzprüfungen durchgeführt und ergaben bemerkenswerte Ergebnisse. Die übrigen Fragestellungen wurden deskriptiv ausgewertet und die Signifikanz festgestellter Veränderungen im zeitlichen Verlauf (vor allem in den Hypothesen $\mathrm{H} 1.1$ bis $\mathrm{H} 1.3$, sowie $\mathrm{H} 2.1$ und $\mathrm{H} 2.2$ ) somit nicht geprüft. Allerdings können die deskriptiv dargestellten Veränderungen von Problemen und Zielen sowie der Wahrnehmung und Bewältigung angegebener Probleme wichtige Tendenzen aufzeigen, welche für den Erfolg der angewandten Psychotherapien sprechen.

Die statistische Aussagefähigkeit der vorliegenden, nach dem Kategoriensystem und der Mastery-Awareness-Scale kodierten Daten zu überprüfen, ist problematisch, da diese verwendeten Rating-Systeme kein Intervallskalenniveau besitzen und eine Abhängigkeitsstruktur der Messungen zur Darstellung der Unterschiede der verschiedenen Messzeitpunkte besteht. Die Problemlage der fehlenden Intervallskalierung ist nicht auf PATH-Daten beschränkt und stellt somit auch andere Untersuchungsinstrumente vor ähnliche Schwierigkeiten.

Darüber hinaus treten bei mit PATH erfragten drei Problemen und Zielen zwangsläufig Mehrfachnennungen auf, welche sich jedoch inhaltlich unterscheiden. In zukünftigen Studien könnten diese Aspekte weiterführend überprüft werden.

Allgemein müssen bei der Interpretation der vorliegenden Untersuchungsergebnisse mehrere Variablen berücksichtigt werden. So können bezüglich der PATH-Daten neben den drei durch die Patienten genannten wichtigsten Problemen und Zielen auch andere Probleme vorhanden sein. Diese können jedoch in der Auswertung der PATH-Fragebögen nicht berücksichtigt werden, da dieses Setting nur drei schriftlich verfasste Probleme und Ziele beurteilt. Dies zeigt, dass hier weiterer Raum für zukünftige Forschungsschwerpunkte zu finden sein könnte.

\section{Diskussion der Kategorien „keine Angaben“ und „keine Probleme“/ "keine Ziele“}

Die Kategorie „keine Angabe“ wurde immer dann kodiert, wenn in den zurück gesendeten Antwortbögen der Patienten für ein Problem oder Ziel keine Eintragung vorgenommen wurde. Die Anzahl dieser Kategorie stieg (wie unter 3.9 beschrieben), je weiter vorangeschritten die Studie war. Dies kann seinen Ursprung einerseits in sinkender Motivation bezüglich der Mitarbeit zur Studie, andererseits auch im Therapie- 
erfolg selbst haben. Außerdem schieden (wie unter 3.9 erwähnt) drei Patienten nach Therapieende aus der Studie aus, so dass sie nicht an den Katamnese-Befragungen teilnahmen.

Gelegentlich gaben Patienten bei der Frage nach den drei Problemen und Zielen an, dass sie „keine Probleme“ oder "kein Ziel“ hätten. Auch solche Aussagen können als mangelnde Motivation seitens der Studienteilnehmer gewertet werden - schließlich ist es weniger aufwändig, dies zu schreiben, als sich aktuelle Probleme oder Ziele zu überlegen und zu formulieren. Die Angaben der Patienten könnten jedoch auch als Zeichen einer Erleichterung insofern gesehen werden, dass aktuelle (Krankheits-) Probleme in den Hintergrund treten und alltägliche Probleme demgegenüber als nicht "nennenswert" erlebt werden.

Es ist folglich von großer Bedeutung, diese beiden diskutierten Kategorien differenziert zu betrachten, da die Aussage, aktuell unter keinem Problem zu leiden, einem besseren Therapie-Ergebnis entsprechen kann, als keine Angabe zu machen.

Um dem Problem fehlender Daten zu begegnen könnte eine Erweiterung des PATHFragebogens zukünftig darin liegen, Patienten, die aktuell keine Probleme und Ziele angeben, jedoch weiter an der Studie teilnehmen, eine zusätzliche Antwortmöglichkeit im Form eines kurzen, ankreuzbaren Items anzubieten. So könnten fehlende Daten (im Sinne nicht ausgefüllter Fragebögen) reduziert werden. Dies scheint besonders bei der Evaluation von Kurzeittherapien sinnvoll zu sein.

\subsection{Diskussion der Ergebnisse im Einzelnen}

Es wurden Veränderungen der Problem- und Zielinhalte (z.B. im Zusammenhang mit Angst oder körperlichen Beschwerden), der Problembewältigung und -wahrnehmung sowie des Leidensdrucks und der Zielverwirklichung festgestellt.

Ebenso wie in der Untersuchung von Leichsenring et al. (2009) und Winkelbach (2007) bestätigt sich auch in dieser Auswertung, dass VT und SET beide zur Verbesserung vor allem des Hauptsymptoms Angst führen.

\subsubsection{Diskussion von Hypothese H1.1}

„Ziele und Probleme verändern sich im Therapieverlauf.“ 
In deskriptiver Darstellungsform kann deutlich gezeigt werden, dass sich im Kurzzeittherapieverlauf Probleme und Ziele der Patienten in erheblichem Umfang verändern. Das zentrale Problem Angst sowie das Ziel, Angst zu bewältigen (Kategorie P1 bzw. Z1 des Kategoriensystems), werden im Laufe der Behandlung rasch seltener angegeben. Dies ist von großer Bedeutung, da alle Studienteilnehmer an GAS litten. Somit verdeutlicht das Ergebnis den Erfolg der Therapien bezüglich dieses Störungsbildes (vorerst unabhängig vom Therapieverfahren). Leichsenring et al. (2005) weisen darauf hin, dass Veränderungen von Zielen auch darauf zurückzuführen sein können, dass sich Patienten im Therapieverlauf sicherer fühlen und Widerstand aufgeben. Ziele der Patienten könnten somit, wie bereits erwähnt, auch Wegweiser für den Behandler sein und negative therapeutische Entwicklungen verhindern.

Die Veränderung von Problem- und Zielinhalten wird anhand der Entwicklung von Kategorie „körperliche Beschwerden“ (P1) des Kategoriensystems ausführlich beschrieben. Je fortgeschrittener die Behandlung, desto seltener wurden körperliche Beschwerden als Problem angegeben. Dies zeigt, dass Patienten in differenzierter, selbstreflektiver Form in der Lage sind, Auskunft über ihre aktuellen Befindlichkeiten zu geben. Bemerkenswert ist zudem, dass bereits nach rund der Hälfte der Therapiedauer (Zeitpunkt t2 = 12. Therapiesitzung) die körperlichen Beschwerden, welche einen wichtigen Anteil des Leidensdrucks der Patienten einnehmen, reduziert werden konnten. Dass diese Kategorie im Untersuchungszeitraum seltener genannt wird, kann darin begründet sein, dass die körperlichen Symptome der GAS abnehmen, oder dass diese Krankheitszeichen von den Patienten als weniger wichtig erachtet werden.

Ungelöste Probleme und Konflikte, z.B. in Alltag oder Beruf (Kategorie P6 des Kategoriensystems), nehmen im Verlauf der Therapie und im Katamnese-Jahr stetig zu. Dies kann als eine gegen Ende der Therapie (auch durch den Rückgang der Angstsymptomatik) ermöglichte, zunehmende Fokussierung auf ungelöste Probleme oder Konflikte des Alltags interpretiert werden. Die Patienten sind nicht mehr so stark durch die generalisierte Angst beeinträchtigt und nehmen wieder mehr am Alltagsgeschehen teil, was die Lösung anderer Probleme in den Vordergrund stellt. Diese Argumentation wird darüber hinaus auch durch das Ergebnis belegt, dass die 
Zielkategorie „äußere Probleme bewältigen“ (Kategorie Z6) im Verlauf ansteigt. Anstatt der der GAS werden die aktuellen Herausforderungen des Alltags von den Patienten stärker gewichtet.

Nicht eindeutig zu beantworten ist, ob Alltagsprobleme im Laufe der Behandlung häufiger angegeben werden, weil die „drängendsten“ Probleme, wie z.B. solche mit Angstinhalten, reduziert werden konnten und in dem Fragebogen nach drei Problemangaben verlangt wird, oder ob neue, bisher noch nicht wahrgenommene Probleme an die Stelle der zuvor genannten treten.

$\mathrm{Zu}$ beachten ist in diesem Zusammenhang, dass eine psychoanalytisch orientierte Therapie (wie das in dieser Studie angewandte SET-Verfahren) nicht in dem Maße wie VT vorher definierte, krankheitswertige Probleme und Ziele fokussiert, da im Therapieprozess unvermeidlich andere Probleme aufgedeckt werden. Das Leiden nimmt, wenn sich Problemschwerpunkte verschieben, somit im Verlauf der Psychotherapie nicht zwangsläufig ab.

Laut Frölich (2009) stellt sich allgemein die Frage, wie eine Veränderung (im Sinne einer Änderung der Kategorie) von Problemen oder Zielen zu bewerten ist. Zu diskutieren bleibt, ob es sich bei einem inhaltlichen Wandel um Progression handelt, ob Probleme tatsächlich gelöst wurden oder schlicht eine Adaptation an bestehende Schwierigkeiten stattfand. Letzteres könnte auch als Ergebnis einer erfolgreichen Therapie bewertet werden, beispielsweise wenn Patienten sich mit (vor allem körperlichen, aber auch psychischen) Einschränkungen arrangieren. Zusätzlich wird zu bedenken gegeben, dass auch veränderte Lebensumstände einen günstigen Einfluss auf Patienten nehmen könnten. Diese Fragestellung kann auch mit dieser Arbeit nicht abschließend geklärt werden. Festzustellen bleibt jedoch, dass die große Anzahl an Veränderungen der Patienten-Aussagen vor allem hinsichtlich der Angstsymptomatik auf einen deutlichen Effekt der Psychotherapie hinweist.

Bei der Beurteilung der geschlechtsspezifischen Ergebnisse müssen einige Voraussetzungen beachtet werden. So muss zur Analyse der Anzahlen einer jeweiligen Kategorie berücksichtigt werden, dass wesentlich mehr Frauen (80\%) als Männer an der Studie teilgenommen haben, was jedoch epidemiologisch zur Geschlechtsverteilung des Krankheitsbildes GAS passt. Wird dies berücksichtigt, zeigt sich bei Frauen ein stärkerer Rückgang in den Problemangaben in Zusammenhang mit Angst, als bei 
Männern. Es könnte angenommen werden, dass Frauen sich unter Umständen intensiver auf die Sicherheit bietende, therapeutische Beziehung einlassen als Männer. Jedoch nehmen auch bei Männern die Probleme im Zusammenhang mit Angst im Untersuchungsverlauf deutlich ab, so dass letztlich beide Geschlechter von der Behandlung zu profitieren scheinen.

Auch bei den sehr bedeutsamen körperlichen Beschwerden lassen sich wiederum geschlechtsspezifische Besonderheiten feststellen, da bei den weiblichen Studienteilnehmern nach anfänglicher Abnahme solcher Beschwerden ein leichter Anstieg zu Therapieende erfolgt. Wie bereits bei der Analyse der Ergebnisse aller Patienten dargestellt, können körperliche Beschwerden jedoch im Untersuchungszeitraum insgesamt bei beiden Geschlechtern deutlich reduziert werden.

Die in dieser Hypothese getrennt nach Geschlecht der GAS-Patienten analysierten Veränderungen der Aussagen im Therapieverlauf sollten auch in Zukunft Gegenstand weiterer Forschungen auf diesem Gebiet sein. Bisher existieren kaum Untersuchungen bezüglich möglicher geschlechtsspezifischer Unterschiede des Störungsbildes GAS (vgl. Becker und Margraf 2007) sowie potentieller Unterschiede in der Behandlung. Zudem könnte beispielsweise weiter untersucht werden, ob das Geschlecht des Psychotherapeuten den Verlauf einer Behandlung beeinflussen kann.

Die Überprüfung dieser Hypothese bezieht sich auf die Auswertung von Problemund Zielangaben der Gesamtgruppe aller Patienten. Interessant wäre eine genauere Untersuchung anhand von Einzelfällen, was jedoch den Rahmen dieser Dissertation übersteigt.

\subsubsection{Diskussion von Hypothese H1.2}

„Die behandelten Patienten formulieren unterschiedliche Probleme und Ziele zu Befragungszeitpunkt $\mathrm{t} 1$, je nachdem, ob sie verhaltenstherapeutisch oder psychodynamisch behandelt werden. Die Patienten wurden zwar randomisiert den Therapieverfahren zugewiesen, jedoch fanden vor Zeitpunkt $\mathrm{t} 1$ bereits drei diagnostische und therapeutische Sitzungen statt.“

Diese Hypothese geht dem frühen Einfluss des Therapieverfahrens nach und fokussiert dabei einen bestimmten Befragungszeitpunkt der Studie zu Behandlungsbeginn: 
$\mathrm{t} 1$ bedeutet, dass die Patienten zur 3. Sitzung den PATH-Fragebogen erhielten. Es fanden folglich bereits Vorgespräche und Therapiestunden statt.

Ein interessanter und zugleich deutlicher Unterschied beider Therapiegruppen zeigt sich hinsichtlich der prozentualen Anteile von Problemen und Zielen im Zusammenhang mit Angst (Kategorie P3 bzw. Z3) zum Befragungszeitpunkt t1. Hier äußern mehr verhaltenstherapeutisch behandelte Patienten Angst, als dies in der psychodynamisch therapierten Patientengruppe der Fall ist. Offensichtlich haben bereits wenige Gespräche mit dem Therapeuten des jeweiligen Therapieverfahrens Einfluss auf die Wahrnehmung der Patienten bezüglich ihrer Ziele und Probleme. Ein Erklärungsansatz könnte sein, dass der Fokus der Behandlung bei VT eher symptombezogen ist, während psychodynamische Therapie auf die zu den Ängsten beitragenden Konflikte (z.B. Beziehungsprobleme) oder die Veränderung von in Beziehungen erworbenen, psychischen Strukturen abzielt und somit viele Problembereiche gleichermaßen gewichtet. Entsprechend werden beispielsweise körperliche Beschwerden (Kategorie P1 des Kategoriensystems), aber noch deutlich häufiger ungelöste Probleme Z.B. des Alltags (Kategorie P6) von SET-Patienten genannt.

Eine weitere große Differenz findet sich bei Zielkategorie „Ruhe und Gelassenheit finden" ( $Z 7$ des Kategoriensystems). Solche Ziele werden ebenfalls häufiger von psychodynamisch behandelten Studienteilnehmern angegeben. Dies könnte so interpretiert werden, dass der emotionalen Stabilität und einer Veränderung des eigenen Erlebens im Sinne von Ruhe und Gelassenheit gegenüber einer Lösungsorientierung bei Angstproblemen größere Bedeutung zugeschrieben wird.

Auch Faller et al. (2002) untersuchten bereits im diagnostischen Interview den Einfluss von Interaktion zwischen Patient und Behandler. Sie beschreiben psychodynamisch orientierte Therapeuten als stärker auf Gefühle und unterdrückte Erfahrungen fokussiert, wohingegen verhaltenstherapeutisch behandelnde Therapeuten an Problemlösungen und der Veränderung maladaptiver Verhaltensweisen arbeiten. Diese unterschiedlichen Arbeitsverfahren beeinflussen wiederum die Wahrnehmung der Patienten bezüglich vordringlicher, in der Behandlung zu bearbeitender Fragestellungen. Sie lernen, wofür sich ihr Therapeut interessiert und richten ihre Aufmerksamkeit darauf. Dies zeigt sich in unseren Ergebnissen bereits nach den ersten drei Sitzungen. 


\subsubsection{Diskussion von Hypothese H1.3}

"Je nach Therapieverfahren (verhaltenstherapeutisch oder psychodynamisch/ SET) werden im Therapieverlauf unterschiedliche Probleme und Ziele genannt."

Die bisher unter 4.3.2 diskutierten Ergebnisse lassen sich auch im weiteren Therapieverlauf bezüglich der Problem- und Zielinhalte erkennen. In beiden Gruppen findet eine deutliche Abnahme von Angst im Laufe der Zeit statt. Jedoch zeigt der Therapieverlauf eine stets häufigere Angabe von Angstproblemen (Kategorie P3) in der verhaltenstherapeutisch behandelten Patientengruppe. Wie bereits erwähnt, könnte ursächlich eine stärkere Fokussierung des Symptoms Angst in der VT sein. Dafür könnte auch sprechen, dass zu Beginn der Therapie, wie auch zur KatamneseBefragung ein Jahr nach Therapieende, eine häufigere Angabe von Zielen, Angst zu bewältigen (Kategorie Z3), bei verhaltenstherapeutisch behandelten Patienten zu verzeichnen ist. Interessanterweise gleichen sich diese Ziele in den Prozentsätzen beider Therapiegruppen zu den Zeitpunkten $\mathrm{t} 2$ bis $\mathrm{t} 4$ an, was dahingehend interpretiert werden kann, dass beide Verfahren im gleichen Umfang die vorrangigen Beschwerden der Patienten bearbeiten.

Einen ebenfalls ähnlichen Verlauf weist Zielkategorie „Ruhe/ Gelassenheit finden“ (Kategorie Z7) auf, da in beiden Vergleichsgruppen solche Ziele im Untersuchungszeitraum prozentual seltener genannt werden. Während der Behandlung erfolgt die Nennung dieser Kategorie häufiger in der SET-Gruppe, was als Beleg für den bereits erläuterten Wunsch nach emotionaler Stabilisierung gewertet werden kann.

Demgegenüber nähern sich Prozentsätze der ungelösten Altagsprobleme (Kategorie P6 des Kategoriensystems) beider Therapiegruppen erst im Laufe der Zeit an. Dies lässt erkennen, dass sie trotz unterschiedlicher Ausgangswerte beider Therapieverfahren aus Sicht der Patienten an Bedeutung gewinnen. Daraus könnte wiederum geschlossen werden, dass das Therapieergebnis inhaltlich ähnlich ausfällt.

Auch äußere Ziele, wie beispielsweise Berufs-, Altags- oder finanzielle Probleme zu lösen, werden in beiden Vergleichsgruppen im Laufe der Untersuchung häufiger angegeben. Wie bei der Analyse von Hypothese H1.1 erwähnt, könnte dies als Beleg einer zunehmenden Altagsorientierung nach Abklingen der durch Symptome der GAS bedingten Probleme gewertet und somit als Wirksamkeit der therapeutischen Intervention verstanden werden. 
Die Nennung interpersoneller Probleme (Kategorie P8) bleibt im Zeitverlauf bei VTPatienten relativ gleich, während sie in der SET-Gruppe einen dynamischen Verlauf nimmt: Zunächst weniger häufig genannt, scheinen derartige Probleme in den Fokus zu rücken, um anschließend nach gelungener Bewältigung seltener genannt zu werden. Zunehmende interpersonelle Probleme können in diesem Zusammenhang auch als Bearbeitung des ZBKT im Rahmen der SET-Therapie verstanden werden. Zur Erläuterung sei an dieser Stelle auf Luborsky (1999) verwiesen: „Bei der Formulierung der Beziehungskonfliktthemen ist die Aufmerksamkeit des Klinikers nicht nur auf das Verständnis der Symptomdeterminanten und des dazugehörenden Leidens gerichtet; es ist ebenso darauf ausgerichtet, die übergeordneten Muster zu erkennen, nach denen der Patient seine Beziehungen zu anderen Menschen gestaltet." (Luborsky 1999, S. 99). „Wenn man bedenkt, dass die Beziehungsprobleme tief im Erleben und Verhalten des Patienten eingebettet sind, dann erscheint es nicht mehr so überraschend, dass sie selbst nach langem und intensivem Durcharbeiten oft immer noch erkennbar sind; glücklicherweise erwirbt der Patient aber geeignete Möglichkeiten der Bewältigung seiner Beziehungsprobleme (...).“ (Luborsky 1999, S. 114).

Da VT stärker auf das manifeste Verhalten und konkrete Lösungsstrategien ausgerichtet ist, werden entsprechend Ziele, bestimmte Freiheiten und Fähigkeiten zu erlangen (Kategorie Z9), ab Erhebungszeitpunkt t2 (12. Therapiesitzung) häufiger von Patienten dieser Therapiegruppe formuliert. Besonders groß ist die Differenz der Vergleichsgruppen in dieser Kategorie zu Behandlungsende (t3).

Ein wichtiger Bereich der Hypothesenprüfung bezieht sich weiterhin auf körperliche Beschwerden. Diese sind eng mit GAS assoziiert, was sich entsprechend der Problemnennungen im Therapieverlauf beider Verfahren zeigt, da die Angaben, ebenso wie die von Angst, sinken. In beiden Untersuchungsgruppen werden derartige Probleme gegen Ende der Behandlung wieder etwas häufiger angegeben. Dies korrespondiert mit folgender Aussage von Leichsenring et al.: „Die Abschlussphase hat zentrale Bedeutung für die Therapie. Die Symptome nehmen häufig wieder zu, da das ZBKT durch den antizipierten Verlust des Therapeuten und durch die antizipierte Nichterfüllung des ZBKT-Wunsches (z.B. Sicherheit, Nähe, Versorgung, Unterstützung, Liebe, Akzeptiertwerden, etc.) aktiviert wird." (Leichsenring et al. 2005, S. 263). 


\subsubsection{Diskussion von Hypothese H1.4}

„Im Therapieverlauf sind systematische Veränderungen festzustellen."

\section{Prüfung mit dem Kategoriensystem:}

Veränderungen von Inhaltskategorien der Problem- und Zielangaben aller Patienten sowie diese im Vergleich der beiden Therapiegruppen wurden bereits im Rahmen der Prüfung von Hypothesen $\mathrm{H} 1.1$ und $\mathrm{H} 1.3$ und der diesbezüglichen Diskussion dargestellt. Sie werden in Zusammenhang mit Hypothese H1.4 als Teilaspekt systematischer Veränderungen verstanden.

\section{Prüfung mit der Mastery-Awareness-Scale:}

Wie Stiles (2006) aufzeigte, verändert sich in einer erfolgreichen Therapie die Problemwahrnehmung in mehreren Abstufungen. Die in dieser Studie gefundenen Ergebnisse können in ähnlicher Hinsicht interpretiert werden.

Die Tatsache, dass Probleme im Laufe der Therapie immer seltener von den Patienten als "nicht greifbar" (Kategorie W1) und häufiger wenigstens als "vage" (Kategorie W2) wahrgenommen werden, zeigt, dass sie durch die psychotherapeutische Behandlung die Fähigkeit erwerben, ihre Probleme besser zu analysieren und als abgrenzbarer zu beschreiben. Die Kategorie „vage Problemwahrnehmung“ hat gegenüber der Kategorie „nicht greifbares Problem“ zwar eine höhere Qualität. Bezüglich der Wahrnehmung von Problemen wäre es allerdings wünschenswert, auch hier zu noch konkreteren Formulierungen der Patienten zu kommen, was möglicherweise nur in längeren Therapien zu erreichen wäre. In Einzelfällen zeigt sich eine leichte Tendenz zur abgrenzbaren Wahrnehmung von Problemen. Im Therapieverlauf werden Probleme außerdem seltener als greifbar beschrieben. Dies könnte darauf hindeuten, dass die Patienten durch die reduzierten Angstsymptome auf neue Probleme stoßen, welche jedoch nicht in dem Maße "fassbar" sind wie Angst.

Auch die Bewältigung angegebener Probleme verändert sich im Laufe der Therapie bezogen auf die Gesamtgruppe der Patienten. Demnach findet ein Rückgang von "Hilflosigkeit" (Kategorie M1) im Umgang mit den Problemen statt, gelegentlich gelingt sogar die „aktive Einflussnahme“ (Kategorie M4a) auf ein Problem. Dies ist 
als positiv zu bewerten, da die Patienten im Umkehrschluss mehr Bewältigungsmöglichkeiten im Laufe der Therapie erlangen. Jedoch ist ein Anstieg der Hilflosigkeit gegenüber Problemen ab Therapieende (t3), vor allem aber ab t4 (sechs Monate nach Therapieende) festzustellen. Der Übergang, den in der Therapie erlernten Umgang mit Problemen nach Therapieabschluss alleine umsetzen zu müssen, scheint einigen Patienten schwer zu fallen. Eventuell könnte hier, wie bereits in der Diskussion der vorherigen Hypothese ausgeführt, auch die Trennung vom Therapeuten oder der therapeutischen Situation eine Rolle spielen.

Der Vergleich beider Behandlungsverfahren ergibt, dass Probleme in beiden Therapiegruppen im Verlauf seltener als „nicht greifbar" beschrieben werden. Von den SET-Patienten werden die angegebenen Probleme zu den Zeitpunkten t1 (Therapiebeginn), t3 (Therapieende) und $\mathrm{t} 4$ (ein halbes Jahr nach Therapieende) häufiger als "vage“ (Kategorie W2) wahrgenommen. Dies könnte erneut so interpretiert werden, dass psychodynamische Therapieverfahren wie SET eher struktur- und weniger symptomorientiert sind. Angaben von weniger deutlich greifbaren Problemen könnten in der analytischen Behandlung als Ergebnis einer Regression verstanden werden, in der die Patienten sich zunächst durch die Komplexität ihrer Befindlichkeit eher irritiert zeigen. Betrachtenswert erscheint die Umkehr des Ergebnisses zum Zeitpunkt t5: Ein Jahr nach Therapieende beschreiben mehr verhaltenstherapeutisch behandelte Studienteilnehmer Probleme als "vage“, sowohl im Vergleich zum vorherigen Erhebungszeitpunkt, als auch in Bezug auf die SET-Vergleichsgruppe. Das spricht dafür, Langzeiteffekte von Psychotherapie differenzierter zu untersuchen.

Als "abgrenzbar" (Kategorie W4a) werden Probleme insgesamt selten gesehen, jedoch etwas häufiger von Patienten aus der psychodynamisch behandlten Therapiegruppe. Dies könnte als Hinweis auf die durch SET vermittelte, selbstrefelxive Technik (siehe 1.1.3) verstanden werden. Da in der SET-Gruppe der höchste Prozentsatz dieser Kategorie an allen jeweils angegebenen Problemen zu Therapieende (t3) auftritt, könnte dies für ein Höchstmaß der Exploration verschiedenster Probleme im therapeutischen Prozess zu diesem Zeitpunkt stehen. Im Therapie-Vergleich der Problembewältigung wird deutlich, dass Hilflosigkeit im Umgang mit angegebenen Problemen mit beiden Therapieverfahren in der Zeit der Behandlung deutlich reduziert werden konnte. Nach Therapieende steigt jedoch 
(ebenfalls in beiden Therapiegruppen) der Anteil derartig erlebter Probleme an. Wie bereits ausgeführt, kann dies ein Hinweis darauf sein, dass in der Therapie erarbeitete Bewältigungsformen nicht immer leicht in die Zeit nach der Therapie „transferiert" werden können.

\section{Prüfung mittels Selbsteinschätzung:}

Ein weiteres zentrales Ergebnis dieser Untersuchung belegt durch Signifikanztests die Abnahme des Leidensdrucks unter zu Behandlungsbeginn (Befragungszeitpunkt t1) angegebenen Problemen im Therapieverlauf sowie die Aufrechterhaltung dieses Ergebnisses auch ein Jahr nach Therapieende. Gleichzeitig steigt das Maß der Zielverwirklichung zu $\mathrm{t} 1$ formulierter Ziele und bleibt ebenfalls nach Behandlungsende auf dem erreichten Niveau. Dieses positive Ergebnis kann so verstanden werden, dass die meisten Patienten sehr von ihrer Behandlung profitieren.

Die Überprüfung auf signifikante Unterschiede der Stärke des Leidensdrucks zu bestimmten Zeitpunkten im Therapieverlauf zeigt, dass zu t1 verhaltenstherapeutisch behandelte Studienteilnehmer durchschnittlich einen höheren Leidensdruck unter zu diesem Zeitpunkt genannten Problemen angeben, was mit der Fokussierung auf zentrale Probleme zusammenhängen könnte. Im Maß des Zielerreichens zu t1 formulierter Ziele können zwischen beiden Kontrollgruppen im Verlauf keine Unterschiede verzeichnet werden, was somit auch als Bestätigung der Wirksamkeit beider Verfahren gesehen werden kann.

Insbesondere ist hervorzuheben, dass die im Zusammenhang mit der Behandlung von GAS-Patienten wichtige Kategorie der „Angst“ (P3/ Z3 des Kategoriensystems) im Verlauf signifikant weniger Leiden verursacht. Gleiches gilt für die Kategorie „körperliche Beschwerden“ (P1/ Z1 des Kategoriensystems). Dabei können keine signifikanten Unterschiede beider Vergleichsgruppen festgestellt werden, was erneut für die Effektivität beider Therapiemethoden sprechen kann. Dies bestätigt die Ergebnisse anderer Untersuchungen, welche ebenfalls eine vergleichbare Effektivität beider Verfahren bezüglich der Besserung von Angst bei GAS-Patienten feststellen (Winkelbach 2007, Leichsenring et al. 2009).

Einschränkend bei der Bewertung dieser Hypothesen-Prüfung ist jedoch der relativ geringe Stichprobenumfang des Gruppenvergleiches der Patienten zu berücksichtigen, die zum Erhebungszeitpunkt t1 Angst bzw. körperliche Beschwerden nannten. 


\subsubsection{Diskussion von Hypothese H2.1}

„Es gibt Prädiktoren, die für einen guten oder eher mäßigen Therapieverlauf sprechen."

Bezüglich möglicher Prädiktoren für einen positiven oder negativen Therapieverlauf müssen einige Überlegungen berücksichtigt werden. Wenn solche Prädiktoren identifiziert werden (beispielsweise inhaltlich in Form von bestimmten Problem- oder Zielangaben, oder als soziodemographische Merkmale der Patienten), so bedeutet dies nicht, dass derartige Vorhersagevariablen auch im Einzelfall den Verlauf einer Psychotherapie beeinflussen müssen. Sicherlich werden solche Merkmale z.B. nicht bei allen Patienten mit gutem Therapieerfolg gefunden, ferner haben gewiss nicht alle "Merkmalsträger" zwangsläufig mit einer Psychotherapie Erfolg in Bezug auf ihre Erkrankung. Desweiteren wurde bei der Überprüfung von Hypothese H2.1 nur der eigentliche Therapiezeitraum und nicht das folgende Katamnese-Jahr berücksichtigt.

Dennoch bieten sich zahlreiche, interessante Ergebnisse für eine Interpretation an. Als potentieller Prädiktor eines erfolgreichen Therapieverlaufs lässt sich das Attribut Alter feststellen: Junge Patienten scheinen stärker von der Therapie zu profitieren, da das Durchschnittsalter in der erfolgreichsten Therapiegruppe am geringsten ist. Dies könnte damit begründet werden, dass der Krankheitsverlauf möglicherweise noch nicht so stark chronifiziert ist. Bezüglich Angststörungen im Alter stellen Eidecker und Gerlach (2009) fest, dass sich in der ambulanten psychotherapeutischen Behandlung eher selten Patienten über 60 Jahren befinden. „Ein möglicher Grund dafür könnte in der Unsicherheit der Behandler liegen, inwiefern eine altersspezifische Behandlungsform notwendig ist." (Eidecker und Gerlach 2009, S. 177). Maercker (2003) stellt aktuelle Konzepte zur Alterspsychotherapie vor und betont dabei die Notwendigkeit von "selektiv optimierten Therapiezielen“ und „altersspezifischen Problempräsentationen“ (Maercker 2003, S. 132).

Außerdem wird in der sehr erfolgreichen Gruppe 1 ein durchschnittlich höherer Leidensdruck unter zu t1 genannten Problemen angegeben. Dies könnte seinen Ur- 
sprung darin haben, dass Gruppe 1 durch die größte Abnahme des Leidensdrucks unter zu $\mathrm{t} 1$ formulierten Problemen innerhalb des Therapiezeitraumes definiert ist und somit ein höheres Niveau des Leidensdrucks zu t1 zu erwarten ist. Dieser Aspekt könnte als methodische Einschränkung betrachtet werden und sollte bei einer Generalisierung der Ergebnisse berücksichtigt werden. Wird dies beachtet, könnten die Befunde so verstanden werden, dass der höhere Leidensdruck stärkere Motivation für den Therapieprozess ist, was auch mit der Argumentation von Beutel (2004) übereinstimmt: „Neuere Studien zeigten, dass der Therapieerfolg durch die Kombination aus Schwere der Symptomatik und früher Verbesserung im Behandlungsverlauf vergleichsweise gut vorhergesagt werden konnte." (Beutel 2004, S. 292).

Ein weiterer bedeutender, möglicher Prädiktor sehr guter Therapieverläufe ist die zu Therapiebeginn häufigere Angabe von Problemen im Zusammenhang mit Angst. Demgegenüber werden zu Erhebungszeitpunkt $t 1$ körperliche Beschwerden öfters von Studienteilnehmern mit mäßigem Behandlungsergebnis berichtet. Diese Ergebnisse könnten darauf zurückzuführen sein, dass die Ausrichtung der GASBehandlungsmanuale beider Therapieverfahren primär störungsspezifisch auf Angst und nicht auf verschiedenartige psychosomatische Symptome bezogen ist. In diesem Zusammenhang ist auch zu verstehen, dass in der sehr guten Gruppe 1 (mit hoher Abnahme des Leidensdrucks im Therapiezeitraum) überwiegend Patienten der VT zu finden sind, was für die Effektivität dieses Verfahrens in Bezug auf den Leidensdruck durch GAS zu sprechen scheint. Dem könnte ein von Milrod (2009) dargelegtes Argument zugrunde liegen: Danach fokussiert VT gut die Symptome einer GAS und es sollte in Zukunft die Frage erörtert werden, ob psychodynamische Psychotherapien bei GAS gezielter auf Patienten mit diesem Störungsbild ausgerichtet werden sollten. Eine solche Orientierung sieht Milrod z.B. in der Konzentration auf Kontrollverlust und eigene Autonomie. Auch Winkelbach (2007) stellt fest, dass VT in der Reduzierung des Sorgenverhaltens SET überlegen ist (Sorgen kann als Teil der Angst bei GAS verstanden werden). Der Leidensdruck bei SET-Patienten wird während der Behandlung im Vergleich zu VT-Patienten weniger stark reduziert, da diese Patienten eher in der mittleren und mäßigen Erfolgsgruppe zu finden sind. Dies könnte damit erklärt werden, dass die der GAS zugrunde liegende Problematik durch SET breiter angesprochen wird und es mit dem Durcharbeiten des zentralen Beziehungskonfliktes in der Trennungssituation vom Therapeuten zu einer Zunahme von Symptomen kom- 
men kann. Im Rahmen anderer Arbeiten (Leichsenring et al. 2009, Winkelbach 2007) konnte jedoch mit unterschiedlichen Instrumenten, welche nicht nach der mit PATH erhobenen Reduktion des Leidensdrucks beurteilen, gezeigt werden, dass beide Therapieverfahren für GAS-Patienten eine effektive Behandlung darstellen.

Wenig differenzierte Problemwahrnehmung und geringere Bewältigungsmöglichkeiten zu Therapiebeginn sprechen eher für mäßige Behandlungsverläufe, während eine "greifbare“ Problemwahrnehmung in der erfolgreichen Gruppe 1 häufiger feststellbar ist. Dieses Ergebnis kann mit dem zur Verfügung stehenden zeitlichen Rahmen einer Kurzzeittherapie in Verbindung gebracht werden: Möglicherweise wäre das Resultat der Senkung des Leidensdrucks in Gruppe 3 mit mäßigem Therapieverlauf noch besser, wenn die Behandlung fortgesetzt worden wäre.

\subsubsection{Diskussion von Hypothese H2.2}

„Die in Hypothese H2.1 ermittelten Prädiktoren für den Erfolg von Therapieverläufen sind innerhalb der beiden untersuchten Therapiegruppen (supportiv-expressive Therapie (SET) und Verhaltenstherapie) unterschiedlich verteilt."

Wie bereits in der Überprüfung von Hypothese H2.2 erläutert, haben die Untergruppen der beiden Therapiegruppen verschiedene Einteilungsgrenzen nach der Abnahme des Leidensdrucks unter zu Befragungszeitpunkt t1 angegebenen Problemen im Verlauf der Behandlung. Daher wird die Verteilung möglicher Prädiktoren innerhalb der Sub-Gruppen gesondert für beide Therapieverfahren beurteilt.

Werden die beiden verhaltenstherapeutisch behandelten Untergruppen 1 (guter Therapieverlauf) und 2 (mittlerer und mäßiger Therapieverlauf) miteinander verglichen, so ergibt sich folgendes Bild: Erfolgreiche VT-Patienten der Untergruppe 1 sind jünger als die der VT-Untergruppe 2, haben zu $\mathrm{t} 1$ einen maximalen Leidensdruck und leiden öfter unter Angst. Sie beschreiben ihre Probleme als "greifbar" und haben dabei eher geringe Bewältigungsmöglichkeiten, jedoch mehr als in VT-Untergruppe 2. In dieser weniger erfolgreichen Vergleichs-Untergruppe 2 finden sich demgegenüber Patienten mit "nicht greifbarer" bzw. „vager" Problemwahrnehmung. Sie beschreiben ihren Zustand mit "Hilflosigkeit und Ausweglosigkeit“, wobei die geschilderten Probleme eher interpersonellen und depressiven Beschwerden zuzuordnen sind. 
Dies könnte, wie in der Diskussion von Hypothese H2.1 bereits erklärt, bedeuten, dass die vorwiegende Fokussierung auf Angst sowie die zu t1 vorhandene, differenziertere Problemwahrnehmung mit den Mitteln der VT erfolgreich gelingt.

Werden hingegen die Erfolgsuntergruppen der psychodynamisch behandelten Patienten näher betrachtet, ist folgende Verteilung feststellbar: Erfolgreiche SETPatienten (Sub-Gruppe 1) sind jüngeren Alters als diejenigen der SET-Untergruppe 2 , sie geben zu Befragungszeitpunkt t 1 vermehrt depressive Beschwerden und Angst an, nehmen diese als "greifbar" wahr und zeigen dabei größere "Hilflosigkeit“. Aus den Problembeschreibungen einiger Patienten geht jedoch hervor, dass diese Probleme bereits "aktiv beeinflussbar" sind. Weniger erfolgreiche SET-Patienten der Untergruppe 2 (mäßiger Therapieverlauf) leiden oftmals unter körperliche Beschwerden, nehmen Probleme öfter als „vage“ wahr und haben diesen gegenüber häufiger "noch keine Bewältigungsmöglichkeit". Interessanterweise unterscheiden sich beide SETUntergruppen nicht hinsichtlich des Leidensdrucks zu Befragungszeitpunkt t1.

Psychodynamische Therapie scheint daher geeignet, GAS-Patienten mit zusätzlich depressiver Symptomatik zu Therapiebeginn (t1) sowie Patienten, die unter großer Hilflosigkeit leiden, erfolgreich zu behandeln. Dies spricht für die Indikation von SET bei komplexeren Störungen.

Diese Ergebnisse sollten durch weitere Forschung validiert werden, da sich klinische Konsequenzen, wie z.B. die Indikationsstellung bestimmter Therapieverfahren für die Behandlung von GAS, daraus ableiten lassen könnten.

\subsection{Ausblick}

Die vorliegende Arbeit zeigt, dass sich Probleme und Ziele sowie die Problemwahrnehmung und -bewältigung im Rahmen einer Kurzzeittherapie von GAS-Patienten verändern. Zum Teil ergeben sich dabei Unterschiede je nachdem, mit welchem Therapieverfahren die Patienten behandelt wurden. Beide Behandlungsmethoden waren jedoch in der Lage, den Leidensdruck unter anfangs angegebenen Problemen signifikant zu reduzieren, im Besonderen jenen durch Angst oder körperliche Beschwerden ausgelösten Leidensdruck. 
Diese Ergebnisse legen nahe, dass folgende Forschungsfragen weiter verfolgt werden sollten:

- Es wäre lohnenswert, in eine Vergleichsstudie Patienten mit GAS einzubeziehen, die eine länger andauernde oder gruppentherapeutische Behandlung erfahren. Es könnte dabei überprüft werden, ob bestimmte Probleme oder Ziele erst nach längerer Therapie bearbeitet und genannt werden und ob sich darüber hinaus spezifische Indikationen für Langzeittherapien oder für Behandlungen im Gruppensetting finden lassen.

- Außerdem wäre es sinnvoll, mit weiteren empirischen Untersuchungen anhand größerer Stichproben die Ergebnisse dieser Untersuchung zu validieren (vgl. Milrod 2009).

- Gegenstand zukünftiger Forschung könnte zudem die Idee sein, PATHFragebögen parallel zur Befragung der Patienten auch an die behandelnden Therapeuten zu richten. Es könnten so Angaben von Patienten und Behandlern verglichen werden und Aspekte der Qualitätssicherung in der psychotherapeutischen Behandlung weiter erforscht werden. Als eine solche FeedbackMethode könnte PATH somit als gemeinsames Instrument von Patienten und Psychotherapeuten genutzt werden.

- Interessant wäre ferner, PATH auch bei weiteren Störungsbildern und störungsübergreifend anzuwenden. Eine erste solche störungsübergreifende Untersuchung mit PATH wurde bereits im Rahmen einer Studie zur Effektivität psychoanalytischer und tiefenpsychologischer Langzeitpsychotherapie durchgeführt (Frölich 2009). 


\section{Zusammenfassung}

Die vorliegende Dissertation wertet Daten einer von der Deutschen ForschungsGemeinschaft geförderten, kontrollierten und randomisierten Studie über die psychotherapeutische Behandlung von 51 Patienten mit Generalisierter Angststörung (GAS) aus, welche zwischen 2001 und 2007 an der Abteilung für Psychosomatische Medizin und Psychotherapie der Georg-August-Universität Göttingen durchgeführt wurde. In der psychotherapeutischen Therapie wurden dabei zwei unterschiedliche Verfahren eingesetzt: die Patienten wurden entweder verhaltenstherapeutisch (VT) oder psychodynamisch (nach Luborskys supportiv-expressiver Therapie, SET) behandelt. Die Studienteilnehmer wurden dabei randomisiert den beiden Therapiegruppen zugewiesen.

GAS ist ein häufig auftretendes, zu Chronifizierung neigendes Störungsbild, das trotz seiner (auch gesundheitspolitischen) Bedeutung und zunehmender Häufigkeit bisher vergleichsweise wenig untersucht ist.

Zudem ist die Forschungslage insbesondere zu psychodynamischen Verfahren bei der Behandlung von GAS bisher begrenzt.

Die ausgewerteten Daten wurden zu fünf Befragungszeitpunkten mit dem Evaluationsinstrument „PATH“ (Problems and Aims in Therapy) erhoben, welches hier erstmalig bei Patienten mit einem einheitlichen/ definierten Krankheitsbild sowie zum ersten Mal zur Auswertung von Kurzzeitpsychotherapie (ca. 25 bis 30 Sitzungen, meist einmal wöchentlich) eingesetzt wird. Dabei werden wiederholt frei formulierte Angaben der Patienten zu ihren drei wichtigsten Problemen und Zielen sowie des Leidensdrucks und der Zielverwirklichung bezüglich dieser erhalten.

Mittels zweier Auswertungsskalen (dem „Kategoriensystem“ und der "MasteryAwareness-Scale") wurde geprüft, ob und wie sich durch PATH erfasste Probleme und Ziele der Studienteilnehmer im Laufe einer Psychotherapie und in einem einjährigen Nachbeobachtungszeitraum verändern. Damit ist der Schwerpunkt der Untersuchung nicht allein auf das Behandlungsergebnis fokussiert, sondern es wird mit differenzierten Fragestellungen der gesamte Behandlungsprozess aus Sicht der $\mathrm{Pa}$ tienten selbst erfasst. Im Speziellen wurden die Ergebnisse der beiden Therapiegruppen miteinander verglichen. 
In überwiegend deskriptiver Weise wird dargestellt, dass sich Probleme und Ziele im Verlauf der Psychotherapie verändern. Der zentrale Punkt der Selbsteinschätzung bezüglich des Leidensdruckes unter zu Beginn der Behandlung angegebenen Problemen sowie die Verwirklichung anfangs genannter Ziele wurden hinsichtlich signifikanter Veränderungen im Therapieverlauf statistisch geprüft.

Die Hauptsymptome Angst und körperliche Beschwerden werden mit der Zeit in beiden Therapiegruppen seltener angegeben, wobei Angst insgesamt häufiger von verhaltenstherapeutisch behandelten Patienten genannt wird. Auch der Leidensdruck unter zu Therapiebeginn formulierten Problemen insgesamt sowie im Zusammenhang mit Angst und körperlichen Beschwerden kann im Therapieverlauf signifikant gesenkt werden. Nach Therapieende ist er gleichbleibend niedrig, wobei sich (abgesehen von einem höheren Leidensdruck unter allen angegebenen Problemen in der VT-Gruppe zu Beginn der Behandlung) keinerlei signifikante Unterschiede beider Therapie-Vergleichsgruppen ergeben. Zudem werden von den Studienteilnehmern depressive Beschwerden im Verlauf seltener als Probleme angeführt. Demgegenüber steigen mit fortschreitender Zeit „ungelöste Probleme/ Konflikte, z.B. in Alltag oder Beruf“ und Ziele, „äußere Probleme (Beruf, Finanzen, Alltag) zu bewältigen“, was als Teil systematischer Veränderungen der Patienten-Aussagen im Therapieprozess gewertet wird. Besonders zu Anfang der Psychotherapie werden solche Probleme häufiger von psychodynamisch behandelten Patienten beschrieben, ebenso wie das Ziel, „Ruhe und Gelassenheit“ zu finden. Dabei ist zu beachten, dass vor dem ersten Befragungszeitpunkt bereits drei Therapiesitzungen durchgeführt wurden, was möglicherweise Einfluss auf die Fokussierung bestimmter Problem- oder Zielinhalte seitens der Patienten nimmt.

Auch die Wahrnehmung und Bewältigung genannter Probleme wurde analysiert. Immer seltener werden diese als „nicht greifbar“ umschrieben und häufiger (vor allem von SET-Patienten) zumindest als „Vage“ erlebt. Der Anteil „greifbar“ beschriebener Probleme sinkt im Behandlungsverlauf. Bezüglich der Problembewältigung ist insgesamt ein Rückgang von „Hilflosigkeit“ im Umgang mit angegeben Problemen zu verzeichnen. Jedoch kommt es nach Therapieende in beiden Vergleichsgruppen zu einem leichten Anstieg dieser Kategorie. Zunehmend wird von den Patienten „noch keine Bewältigungsmöglichkeit“ gesehen, außerdem werden im Verlauf häufiger Probleme formuliert, die sehr wohl „Handlungs- und Bewältigungsmöglichkeiten“ erkennen lassen. Als „abgrenzbar“ und „aktiv beeinflussbar“ werden Probleme von 
den Patienten zwar insgesamt selten dargestellt, jedoch am häufigsten zu Therapieende.

Basierend auf dem Rückgang des Leidensdrucks unter zu Therapiebeginn genannten Problemen ergibt die Analyse der Patientenangaben dieses Zeitpunktes, dass als mögliche Prädiktoren erfolgreicher Therapieverläufe u. a. jüngeres Alter, verhaltenstherapeutische Behandlung, ein hoher Leidensdruck, Probleme des Inhaltes Angst, eine differenzierte Problemwahrnehmung sowie vorhandene Bewältigungsmöglichkeiten gelten könnten. Die nach beiden Therapieverfahren getrennte Auswertung zeigt zudem, dass in der VT-Gruppe depressive Beschwerden und „nicht greifbare“ Probleme potentielle Prädiktoren eines ungünstigen Behandlungsverlaufs sind. Demgegenüber könnten in der SET-Behandlungsgruppe neben jüngerem Alter - anders als in der erfolgreichen VT-Vergleichsgruppe - auch depressive Beschwerden und Hilflosigkeit im Umgang mit Problemen Prädiktoren für einen erfolgreichen Therapieverlauf sein. Von Patienten angegebener, hoher Leidensdruck ist hier kein Prädiktor eines ungünstigen Behandlungsverlaufs.

Selbst im Rahmen von Kurzzeittherapien lassen sich somit differenzierte Behandlungseffekte sowie die Wirksamkeit beider untersuchten Verfahren nachweisen. 


\section{Literaturverzeichnis}

Becker E, Margraf J: Generalisierte Angststörung: Ein Therapieprogramm. 2. Auflage, Beltz Verlag, Weinheim/ Basel 2007

Beutel ME (2000): Psychodynamische Kurztherapien. Psychotherapeut 4드, 203-213

Beutel ME (2004): Kurztherapie - die (neue) Norm? Psychotherapeut 49, 292-294

Bortz J: Statistik für Human- und Sozialwissenschaftler. 6. Auflage, Springer Medizin Verlag, Heidelberg 2005

Crits-Christoph P, Wolf-Palacio D, Ficher M, Rudick D: Brief supportiveexpressive psychodynamic therapy for generalized anxiety disorder. In: Barber JP, Crits-Christoph P: Dynamic therapies for psychiatric disorders (axis I). Basic Books, New York 1995, 43-83

Crits-Christoph P, Gibbons MB, Losardo D, Narducci J, Schamberger M, Gallop R (2004): Who Benefits from Brief Psychodynamic Therapy for Generalized Anxiety Disorder? Can J Psychoanal 12, 301-324

Dahlbender RW, Erena C, Reichenauer G, Kächele H (2001): Meisterung konflikthafter Beziehungsmuster im Verlaufe einer psychodynamischen Fokaltherapie. Psychother Psychosom Med Psychol 51 (3/4), 176-185

DIMDI (Deutsches Institut für Medizinische Dokumentation und Information): ICD-10-GM 2009, Systematisches Verzeichnis. Deutscher Ärzte Verlag, Köln 2008

Dirmaier J, Koch U, Kawski S, Schulz H (2002): Therapieziele als Qualitätsmanagement - Instrumentarium in der psychosomatischen Rehabilitation. Z Aerztl Fortbild Qualitätssich $\underline{96}$, 25-30 
Eidecker J, Gerlach AL (2009): Kognitiv-verhaltenstherapeutische Behandlung von Angststörungen im hohen Erwachsenenalter. Z Psychiatr Psychol Psychother $\underline{57}$ (3), 177-184

Elliot R, Marck C, Shapiro DA: Simplified Personal Questionnaire Procedure. University of Toledo, Toledo 1999 (www.experiential-researchers.org/instruments/elliott/pqprocedure.html, 24.06.2010)

Faller H, Wagner RF, Weiß H, Lang H (2002): Therapists' Relationships with their Patients in the Intake Interview: An Empirical Comparison of Psychodynamically and Cognitive-Behaviorally Oriented Psychotherapists. J Am Acad Psychoanal 30 (3), $451-461$

Fitzpatrick M, Iwakabe S, Stalikas A (2005): Perspective divergence in the working alliance. Psychother Res 15 (1-2), 69-79

Freyberger H, Schneider W (2001): ICD-10-Diagnostik in der Psychotherapie. Psychotherapeut $\underline{46}, 115-121$

Frölich S: Veränderungen von Problemen und Zielen während Langzeitpsychotherapien: Entwicklung und erste Anwendung eines Kategoriensystems zur Auswertung frei formulierter Patientenangaben. Med. Diss. Göttingen 2009

Grande T, Dahlbender R, Schauenburg H, Stasch M, Cierpka M (2005): Neue Möglichkeiten der Diagnostik dysfunktionaler Beziehungen - die Beziehungsachse des OPD-2. Z Psychosom Med Psychother 51, 403-418

Grande T, Dilg R, Jakobsen T, Keller W, Krawietz B, Langer M, Oberbracht C, Stehle S, Stennes M, Rudolf G (2006): Differential effects oft two forms of psychoanalytic therapy: Results oft the Heidelberg-Berlin study. Psychother Res 16 (4), 470485

Grawe K, Donati R, Bernauer F: Psychotherapie im Wandel. Von der Konfession zur Profession. Hogrefe, Göttingen 1994 
Grosse Holtforth M, Grawe K (2002): Bern Inventory of Treatment Goals: Part 1. Development and first Application of a Taxonomy of Treatment Goal Themes. Psychother Res 12 (1), 79-99

Häcker H, Stapf KH: Psychologisches Wörterbuch. Verlag Hans Huber, Bern 2004

Höfler M: Statistik in der Epidemiologie psychischer Störungen. Springer Verlag, Berlin/ Heidelberg 2004

Hohage R (2001): ICD-10-Diagnosen in der psychotherapeutischen Praxis. Psychotherapeut $\underline{46}, 110-114$

Kiresuk TJ, Sherman RE (1968): Goal attainment scaling: a general method for evaluating comprehensive community mental health programs. Community Ment Health J 4 (6), 443-453

Laplanche J, Pontalis JB: Das Vokabular der Psychoanalyse. Suhrkamp Verlag, Frankfurt am Main 1973

Leibing E, Winkelbach C, Leichsenring F (2003): Die Generalisierte Angststörung: Darstellung eines kognitiv-behavioralen Behandlungsmanuals. Verhaltensther Psychosoz Prax $\underline{35}, 517-529$

Leichsenring F (1996): Zur Meta-Analyse von Grawe und Mitarbeitern. Gruppenpsychother Gruppendynamik 32, 205-234

Leichsenring F, Winkelbach C, Leibing E (2005): Psychoanalytisch-orientierte Fokaltherapie der generalisierten Angststörung: Ein Manual. Psychotherapeut $\underline{50}$, 258264

Leichsenring F, Salzer S, Jaeger U, Kächele H, Kreische R, Leweke F, Rueger U, Winkelbach C, Leibing E (2009): Short-Term Psychodynamic Psychotherapy and Cognitive-Behavioral Therapy in Generalized Anxiety Disorder: A Randomized, Controlled Trial. Am J Psychiatry 166, 875-881 
Luborsky, L: Einführung in die analytische Psychotherapie: ein Lehrbuch. Vandenhoeck \& Ruprecht, Göttingen 1999

Maercker A (2003): Alterspsychotherapie - aktuelle Konzepte und Therapieaspekte. Psychotherapeut $\underline{48}, 132-149$

Milrod B (2009): Psychodynamic Psychotherapy for Generalized Anxiety Disorder. Am J Psychiatry 166, 841-844

Rudolf G: Strukturbezogene Psychotherapie - Leitfaden zur psychodynamischen Therapie struktureller Störungen. 2. Auflage, Schattenauer Verlag, Stuttgart 2006

Rudolf G, Grande T, Oberbracht C (2000): Die Heidelberger Umstrukturierungsskala. Psychotherapeut $\underline{45}, 237-246$

Saß H, Wittchen HU, Zaudig M, Houben I: Diagnostisches uns Statistisches Manual Psychischer Störungen - Textrevision - DSM-IV-TR. Hogrefe Verlag, Göttingen 2003

Senf W, Broda M: Praxis der Psychotherapie - ein integratives Lehrbuch. Georg Thieme Verlag, Stuttgart 2005

Staats H: Problems and Aims in Therapy (PATH). Prüfplan für die Ethikkommission, Universität Göttingen, Göttingen 2000

Staats H: Das zentrale Thema der Stunde. Vandenhoeck \& Ruprecht, Göttingen 2004

Staats H: Unveröffentlichtes Manuskript der Mastery-Awareness-Scale. Universität Göttingen, Göttingen 2009

Staats H, Biskup J, Leichsenring F: PATH - a method for investigating the development of problems and aims in therapy in the Göttingen study on the effectiveness of psychoanalytic psychotherapy. Abstract der Präsentation auf der $5^{\text {th }}$ European 
Conference on Psychotherapy Research, Paper Session 10 S.145, Cernobbio (Como) 04.-07.09.1996

Stiles WB (2006): Assimilation and the process of outcome: Introduction to a special section. Psychother Res 16 (4), 389-392

Trachsel M, Itten S, Stauffer B, Grosse Holtforth M, Hofer D (2008): Erreichung strörungsübergreifender Therapieziele in einer störungsspezifischen ambulanten Gruppenpsychotherapie phobischer Patienten. Psychother Psychosom Med Psychol $\underline{58(9 / 10)}, 359-365$

Wampold BE: The Great Psychotherapy Debate: Models, Methods, and Findings. Lawrence Erlbaum Associates, Mahwah NJ 2001

WBP (Wissenschaftlicher Beirat Psychotherapie, 2009): Methodenpapier des Wissenschaftlichen Beirats Psychotherapie nach § 11 PsychThG, Version 2.7. (www.wbpsychotherapie.de/downloads/Methodenpapier2720090709.pdf, 15.03.2010)

Weiß C: Datenanalyse und Modellierung mit STATISTICA. Oldenbourg Verlag, München 2007

Werling VAC: Darstellung der Ziele und Probleme von Patienten mit Generalisierter Angststörung: Vergleich einer Patientengruppe in psychotherapeutischer Behandlung mit einer Gruppe von Nicht-Patienten. Med. Diss. Göttingen 2010 (in Vorbereitung ${ }^{11}$ )

Winkelbach C: Psychodynamische Kurzzeittherapie und kognitive Verhaltenstherapie bei generalisierter Angststörung: Eine randomisierte, kontrollierte und manualisierte Therapiestudie. Psychol. Diss. Göttingen 2007

Wittchen HU, Hoyer J: Klinische Psychologie \& Psychotherapie. Springer Medizin Verlag, Heidelberg 2006

\footnotetext{
${ }^{11}$ mit freundlicher Genehmigung der Verfasserin
} 
Wittmund B, Wilms HU, Bull N, Angermeyer MC (2003): Zusammenarbeit einer Spezialambulanz mit niedergelassenen Allgemeinmedizinern und Nervenärzten bei der Behandlung von Angsterkrankungen. Verhaltenstherapie 13, 284-290 


\section{Anhang}

\subsection{Abbildungen zu Hypothese H1.1 („Ziele und Probleme verändern sich im Therapieverlauf.")}

\subsubsection{Problem- und Zielangaben aller Patienten, Verteilung auf Inhaltskatego- rien des Kategoriensystems}

Es stellen alle Problem- oder Zielangaben eines Befragungszeitpunktes im Kreisdiagramm $100 \%$ dar.

Abbildung 37: Verteilung aller Problem- und Zielangaben zu den Zeitpunkten t2 bis t4,

Rating mittels des Kategoriensystems
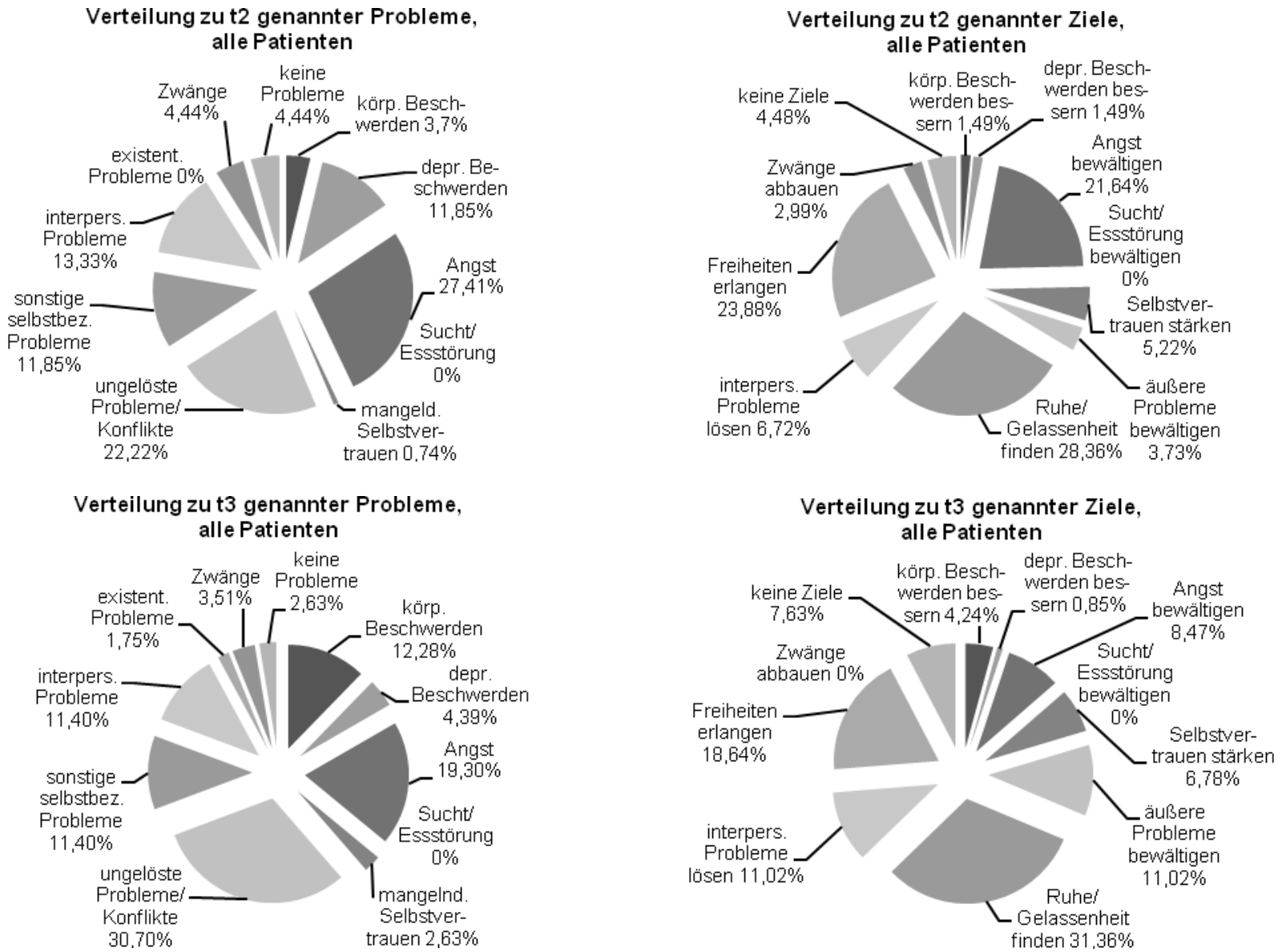

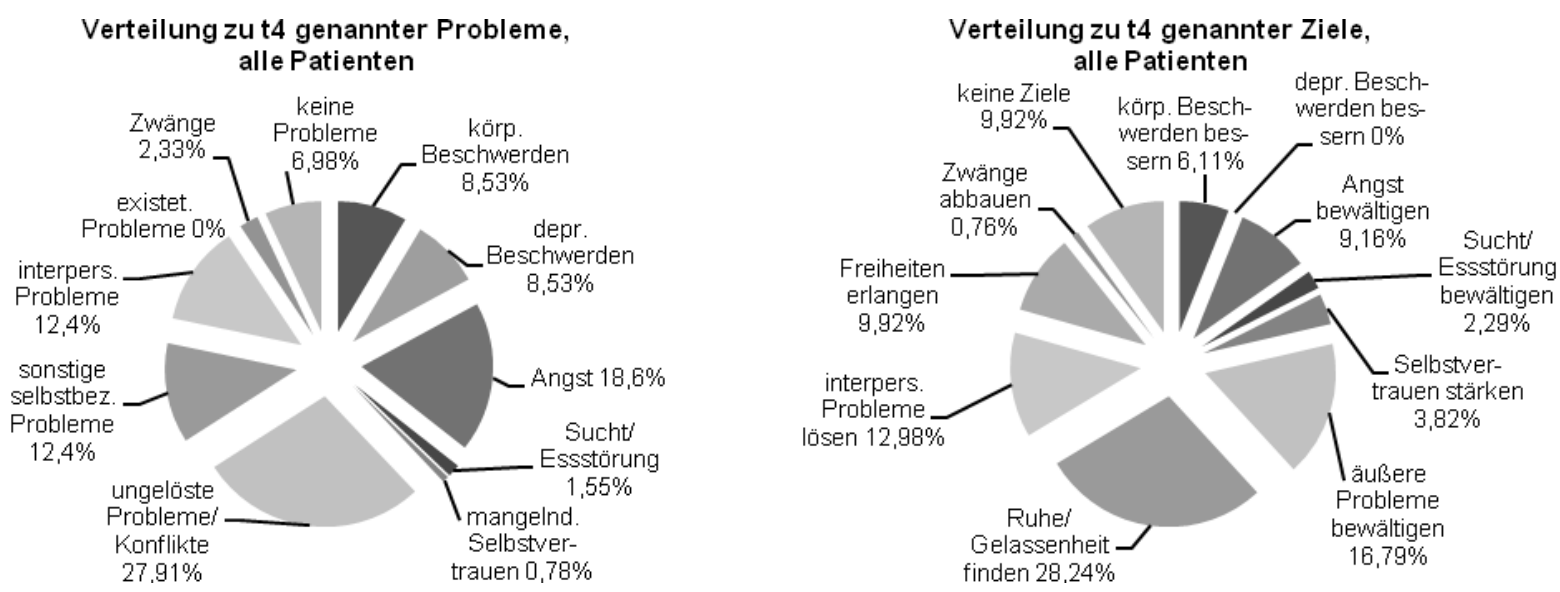

\subsubsection{Problem- und Zielangaben aller Patienten, Verteilung auf Inhaltskatego- rien des Kategoriensystems, Vergleich Frauen und Männer}

Die Prozentsätze beziehen sich auf $100 \%$ als alle zum jeweiligen Zeitpunkt von Frauen oder Männern angegebene Probleme oder Ziele.

Abbildung 38: Verteilung genannter Probleme und Ziele auf Inhaltskategorien des Kategoriensystems, Zeitpunkte $\mathbf{t} 2$ bis t4, Frauen und Männer getrennt
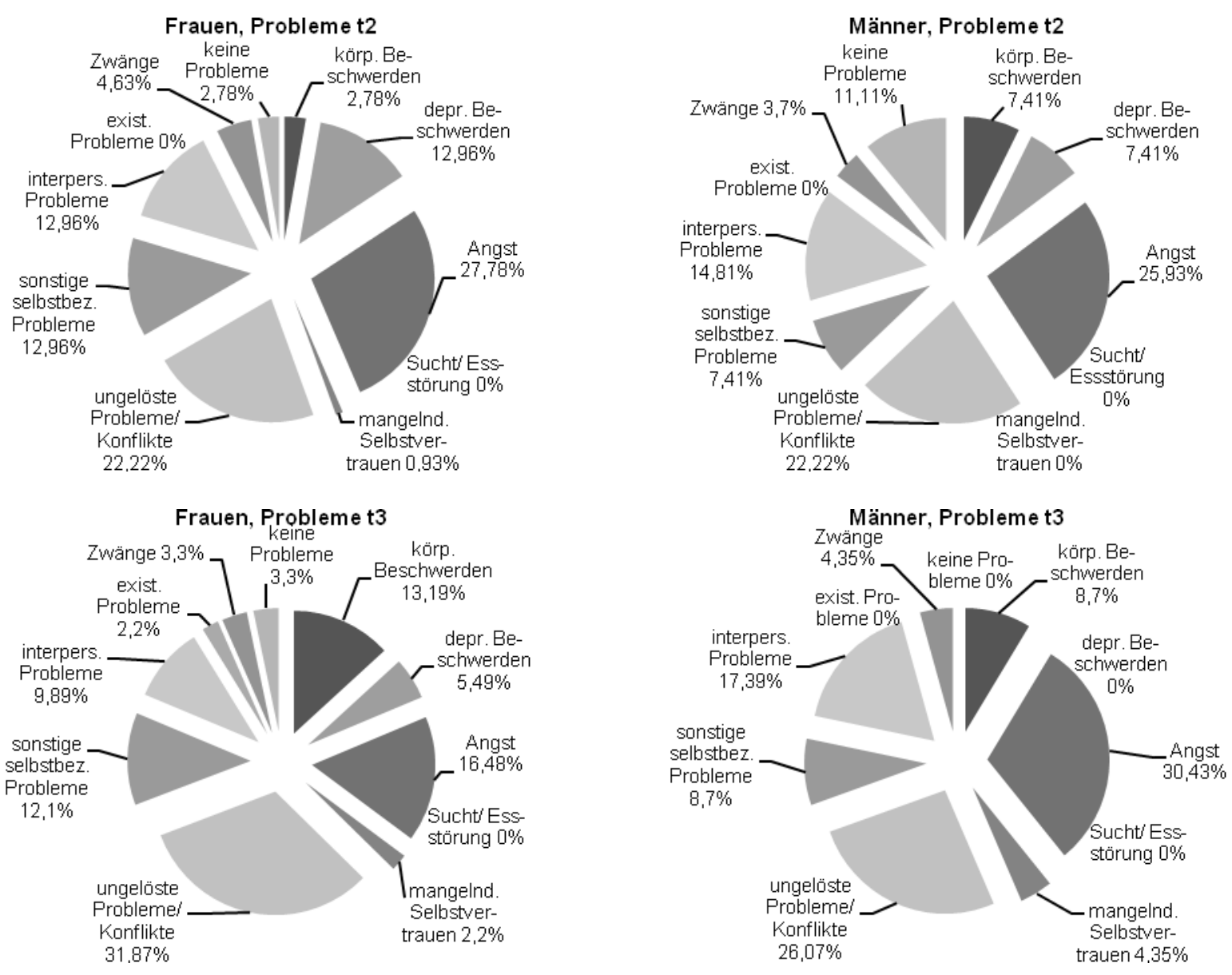

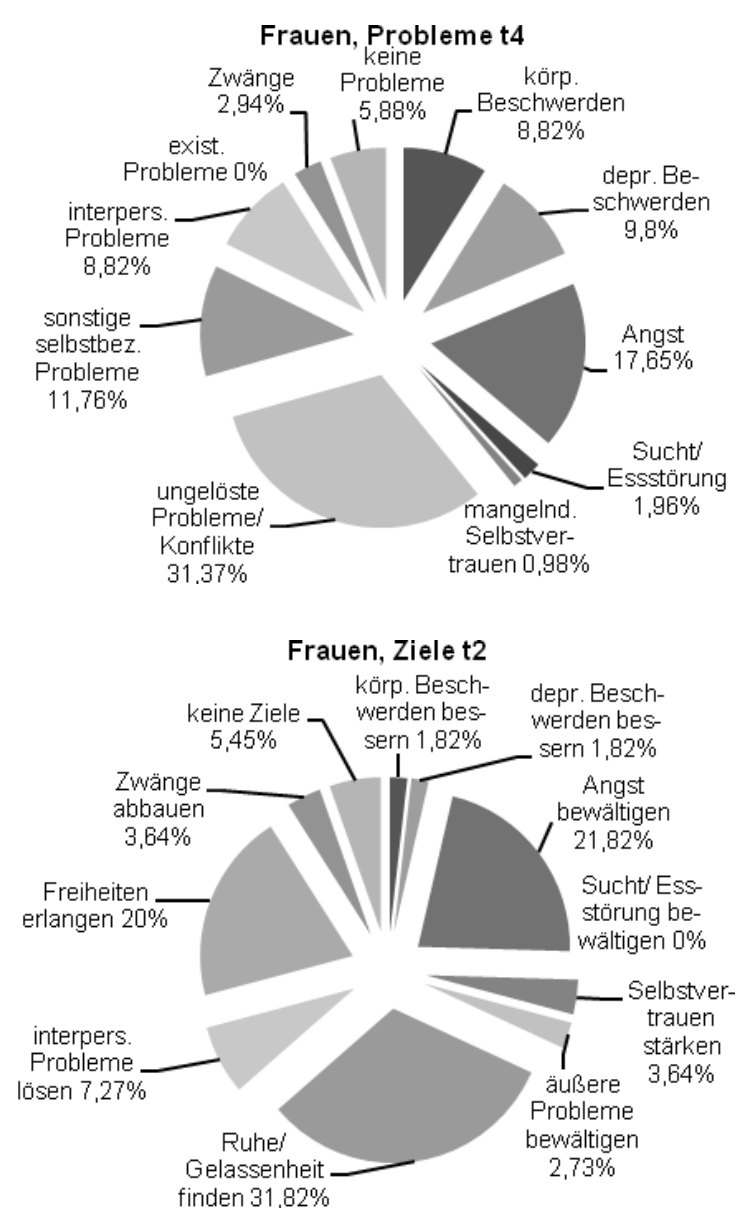

Frauen, Ziele $\mathrm{t} 3$

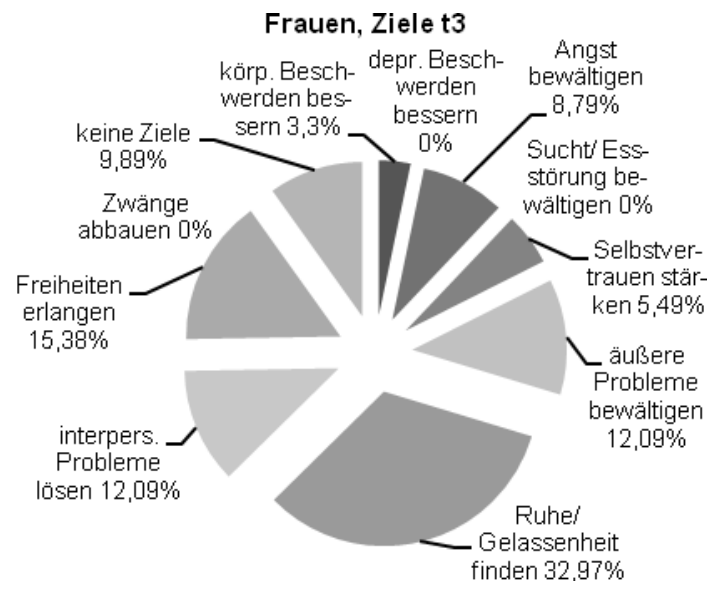

Frauen, Ziele t4

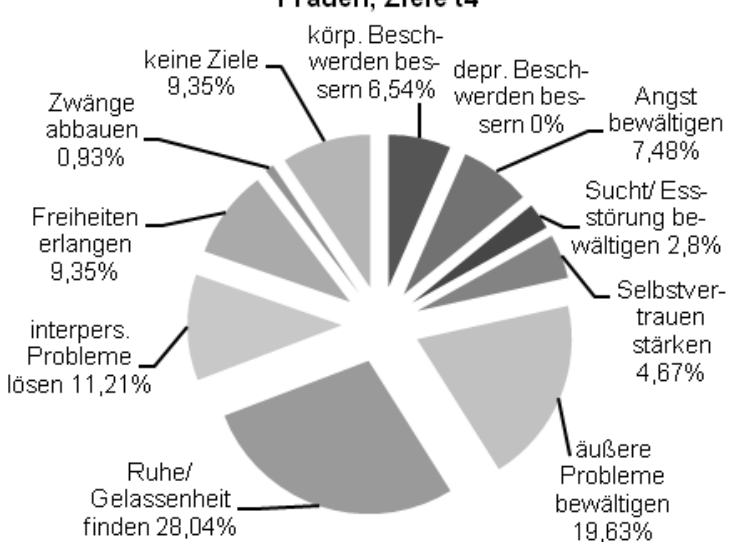

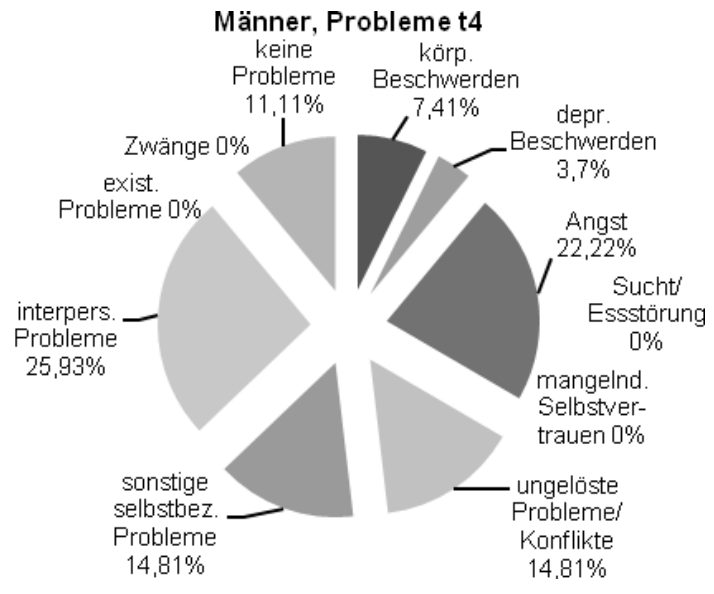

Männer, Ziele t2
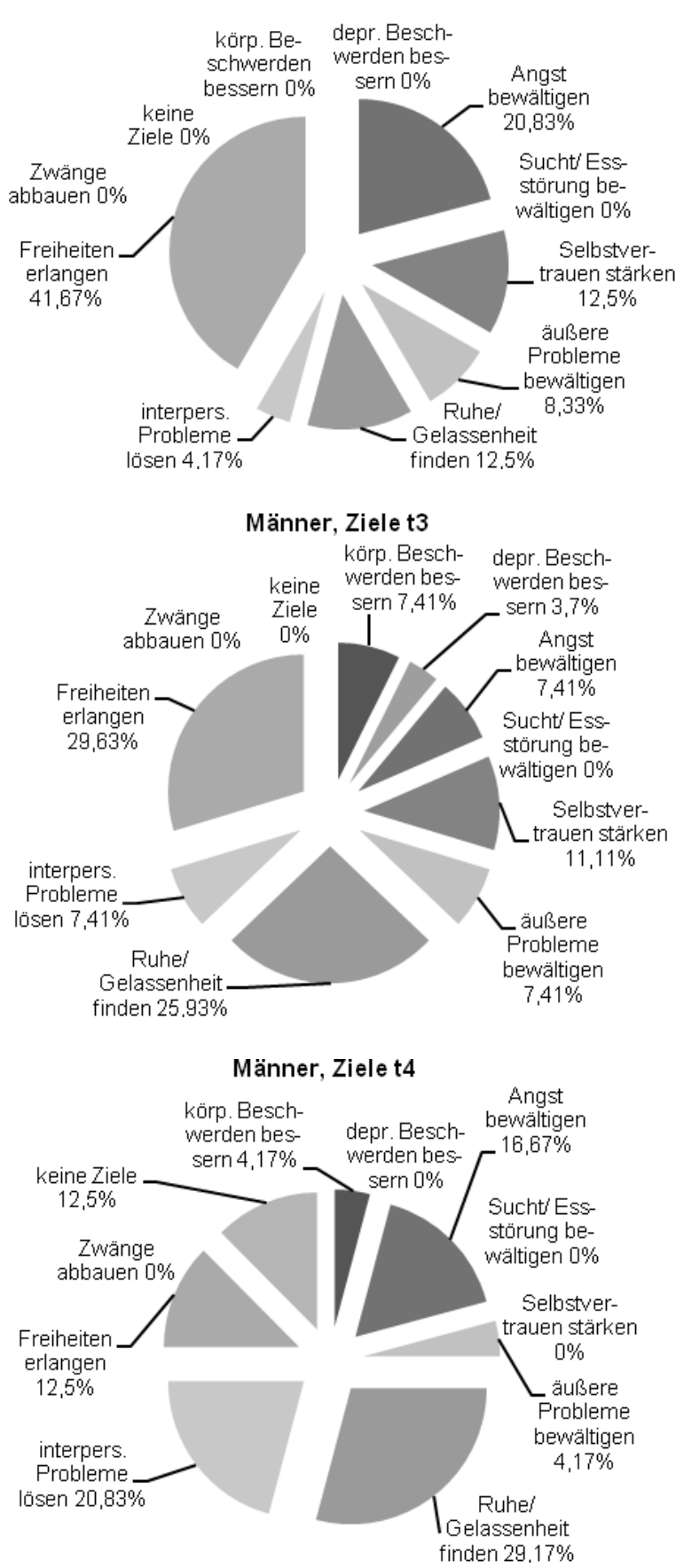


\subsection{Abbildungen zu Hypothese H1.3 („Je nach Therapieverfahren (verhal-}

tenstherapeutisch oder psychodynamisch/ SET) werden im Therapieverlauf unterschiedliche Probleme und Ziele genannt")

$100 \%$ entsprechen jeweils allen in einer Behandlungsgruppe angegebenen Problemen oder Zielen eines Zeitpunktes.

Abbildung 39: Verteilung genannter Probleme und Ziele beider Therapiegruppen auf Inhaltskategorien des Kategoriensystems, Zeitpunkte t2 bis t4
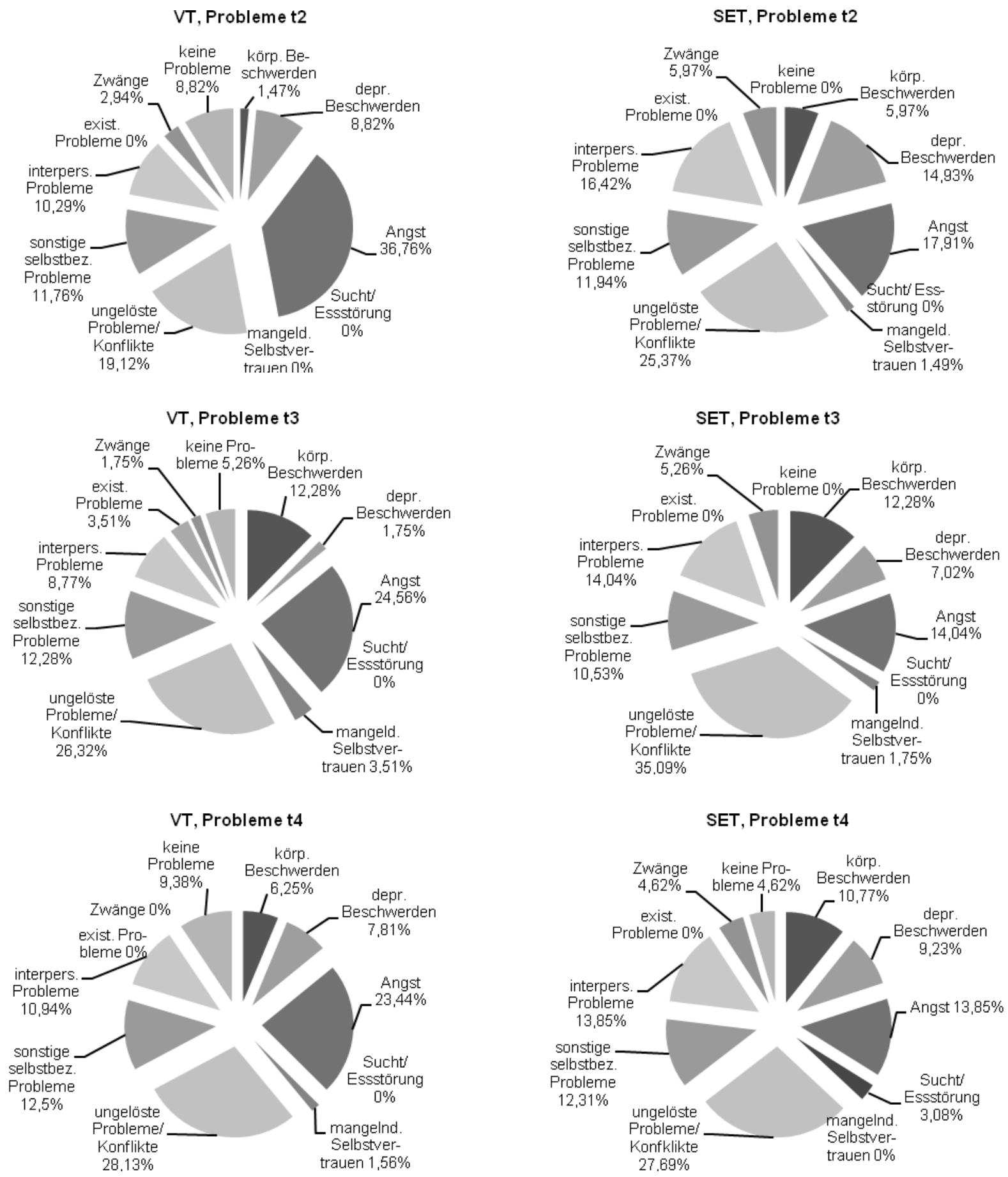
VT, Ziele t2

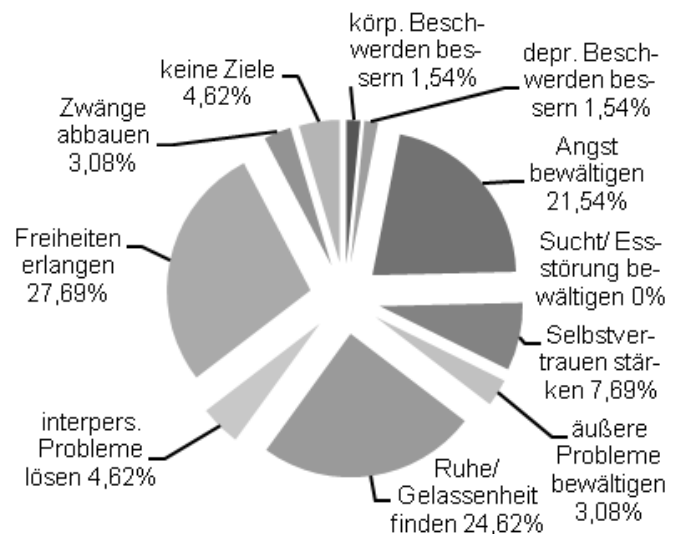

VT, Ziele t3

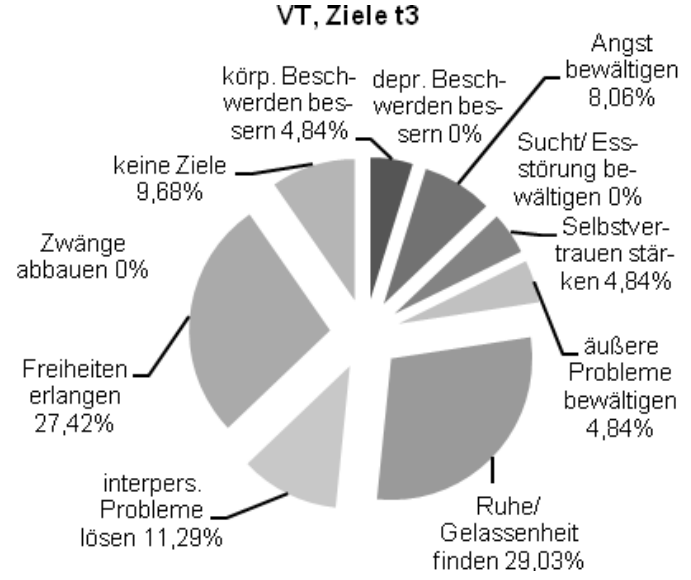

VT, Ziele t4

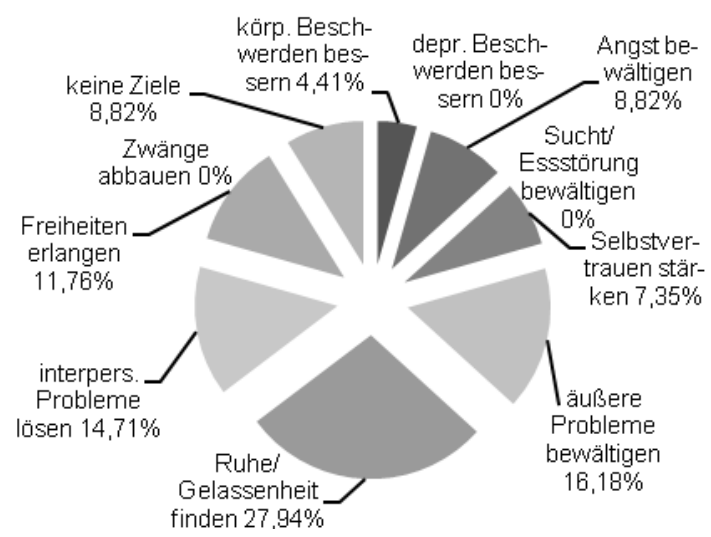

SET, Ziele t2

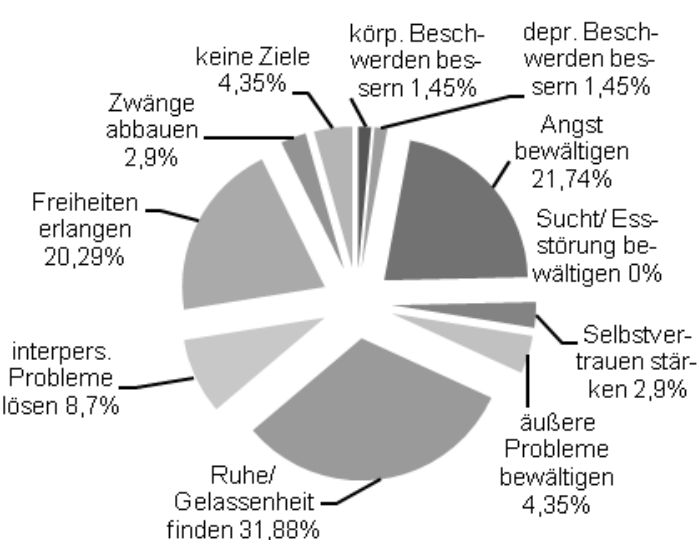

SET, Ziele t3

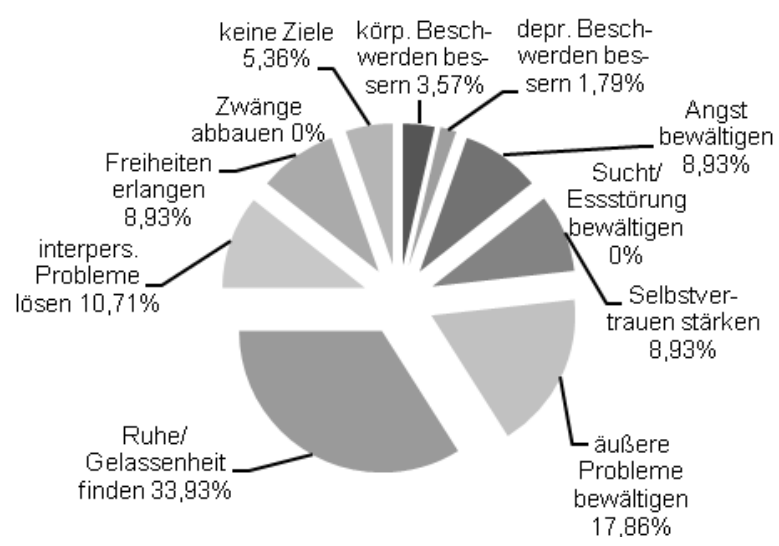

SET, Ziele t4

körp. Besch-

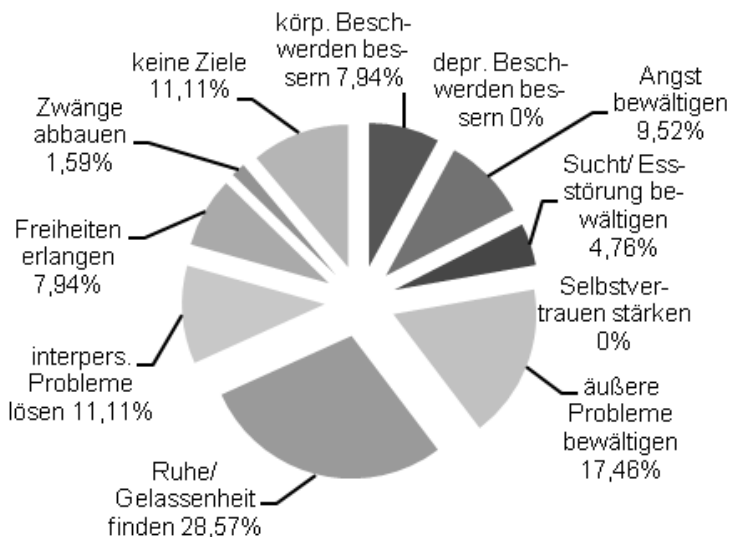

\subsection{Abbildungen, Tabellen und statistische Definitionen zu Hypothese}

\section{H1.4 („Im Therapieverlauf sind systematische Veränderungen festzustel-}

len.")

\subsubsection{Rating von Problemen und Zielen mit der Mastery-Awareness-Scale}


Abbildung 40: Mastery-Awareness-Scale, zeitlicher Verlauf der Problemwahrnehmung, gesamtes Patientenkollektiv

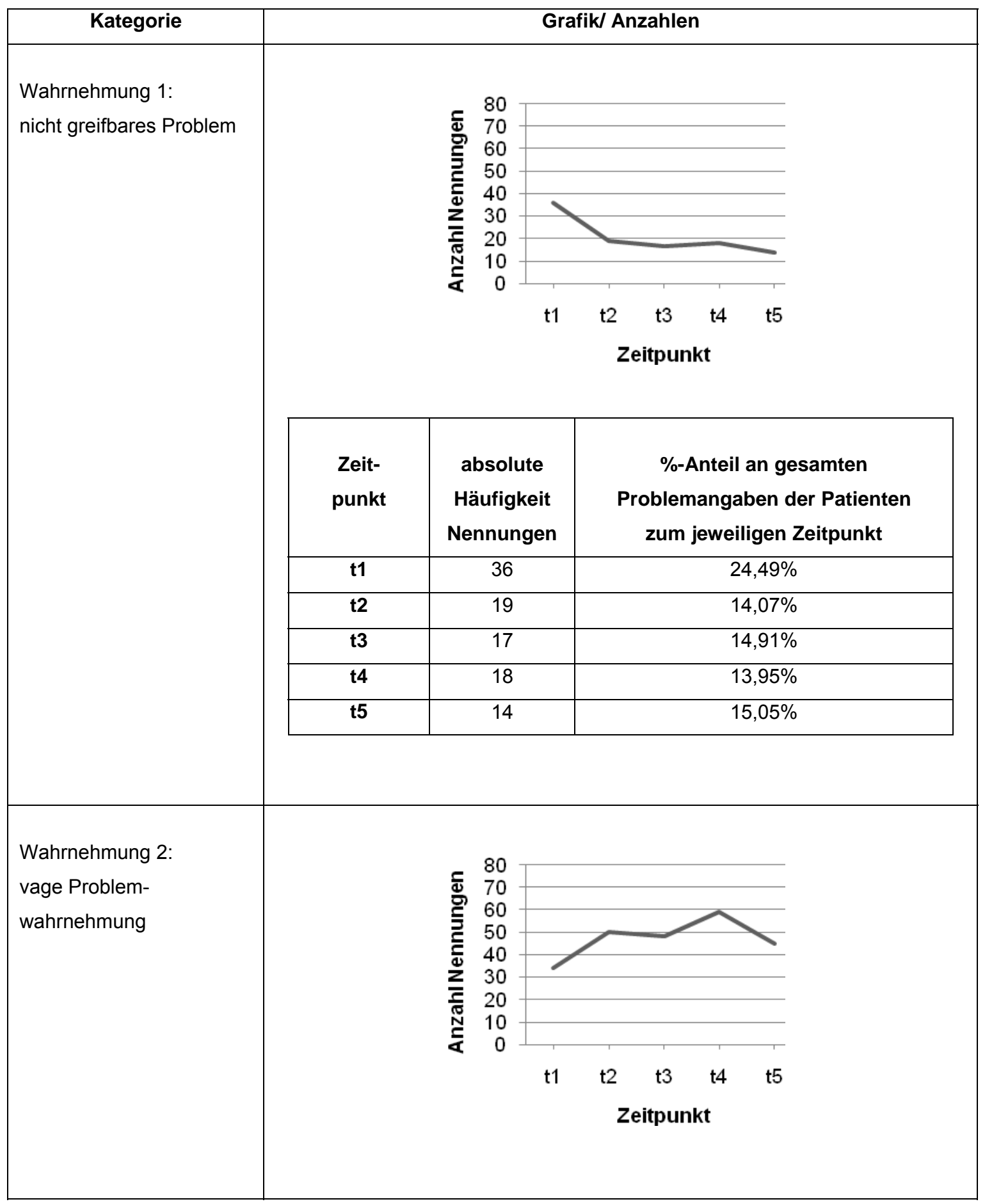




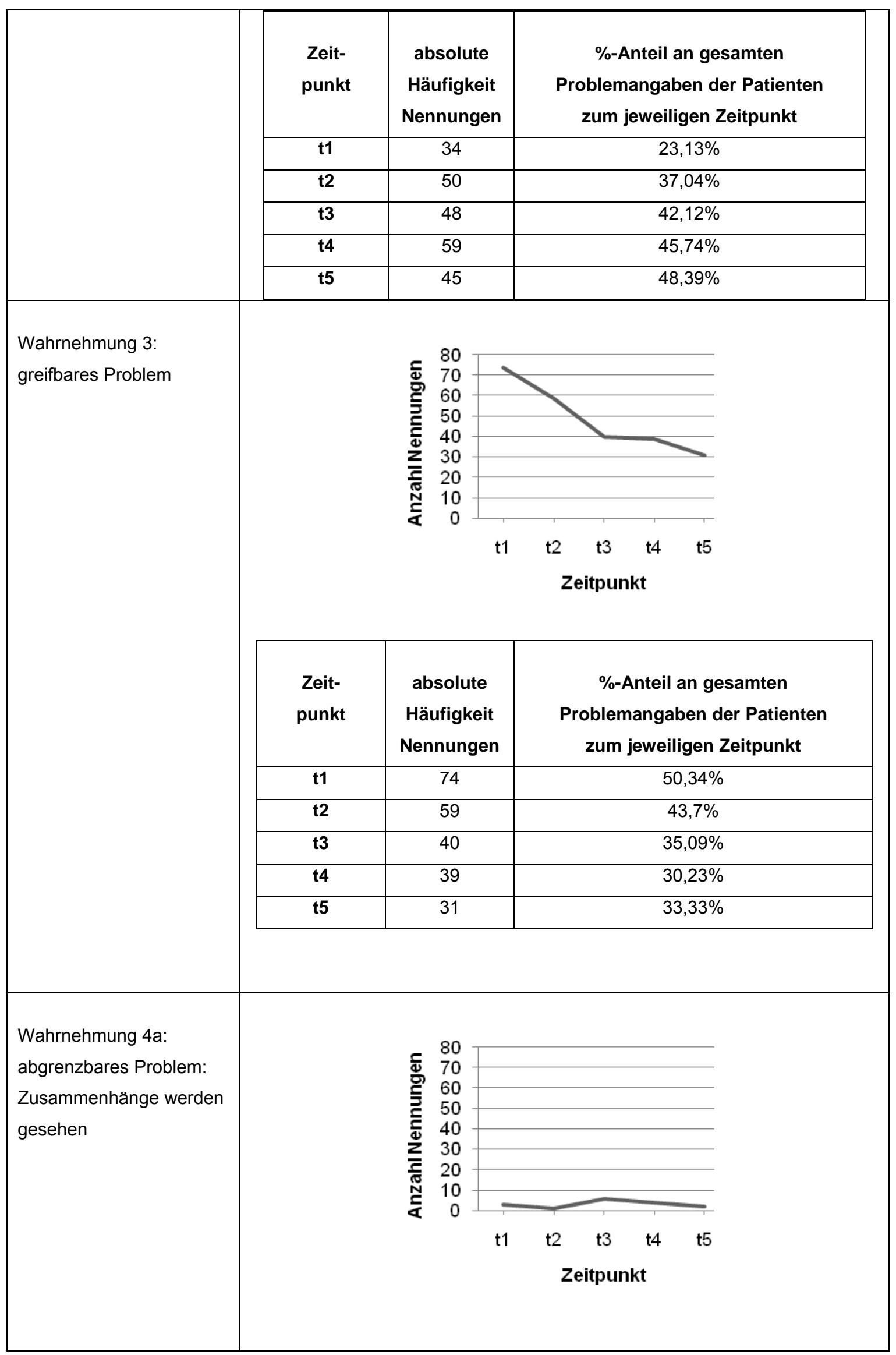




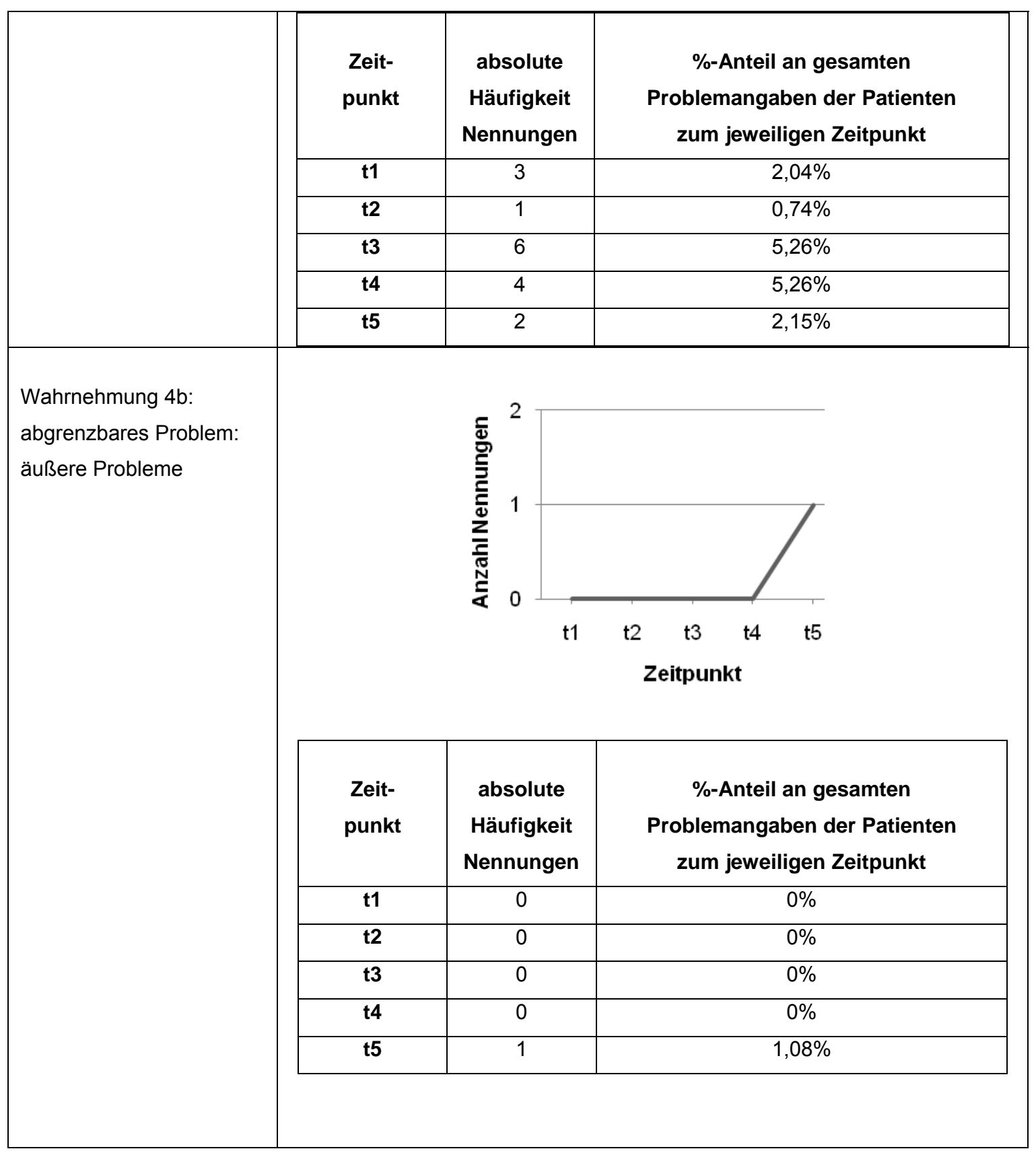

Abbildung 41: Mastery-Awareness-Scale, zeitlicher

Verlauf der Problembewältigung, gesamtes Patientenkollektiv 


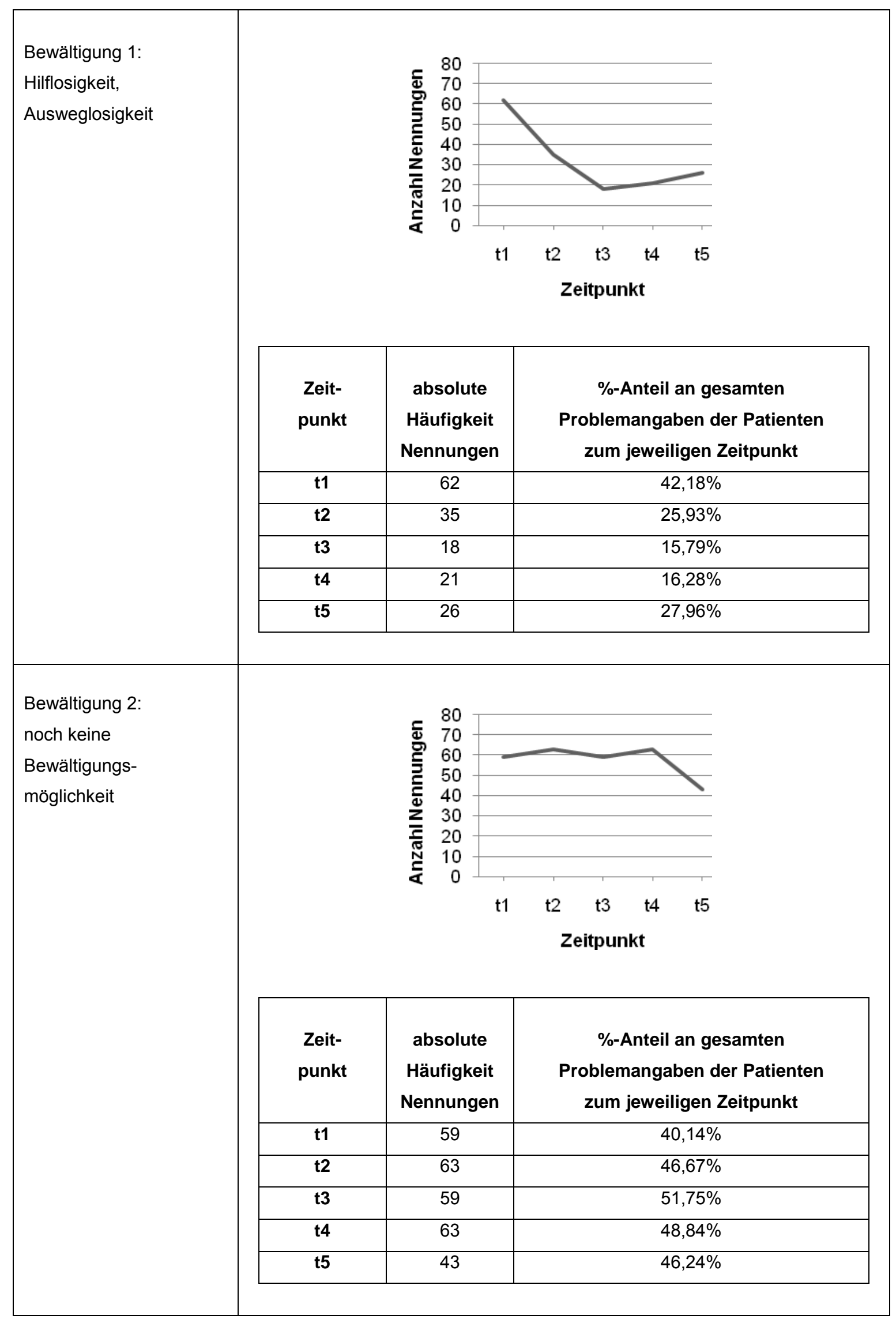




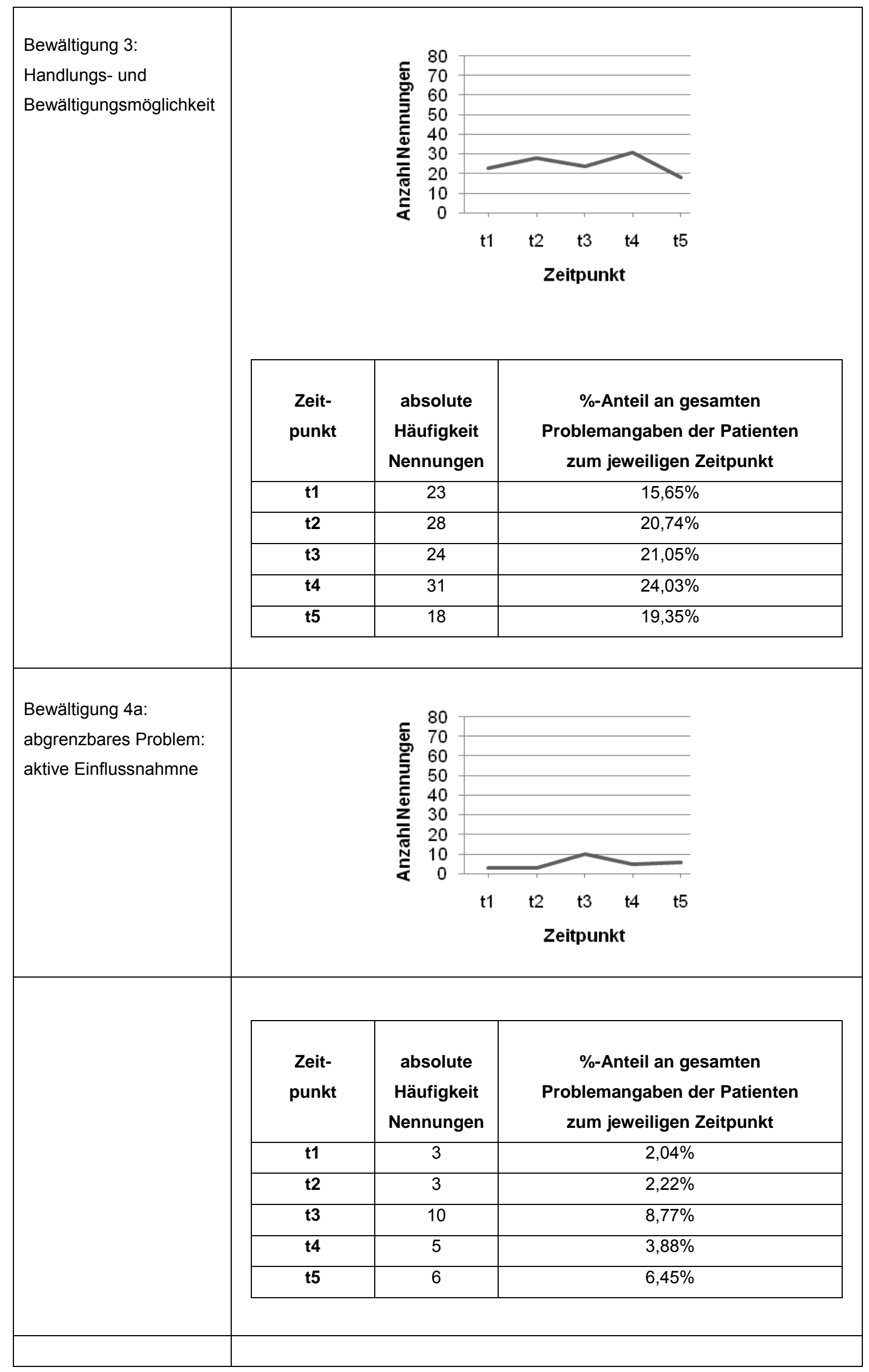




\begin{tabular}{|l|l|}
\hline Bewältigung 4b: & Probleme dieser Kategorie wurden nicht genannt. \\
abgrenzbares Problem: & \\
selbstverständlicher & \\
Umgang mit Problem & \\
\hline
\end{tabular}

Abbildung 42: Mastery-Awareness-Scale, zeitlicher Verlauf der Problemwahrnehmung, Vergleich der beiden Therapieformen VT und SET

Zusätzliche Erklärung am Beispiel von Kategorie W3:

Zum Zeitpunkt $\mathrm{t} 3$ wurden in beiden Therapiegruppen 20 Probleme dieser Kategorie zugeordnet. Da zu diesem

Zeitpunkt in beiden Gruppen gleich viele Problemaussagen vorliegen, ist auch der Prozentsatz an allen Problemen einer Therapiegruppe zu t3 identisch. Anders sieht dies zum Zeitpunkt t5 aus, da in der SET-Gruppe weniger Problemaussagen gemacht wurden (also mehr fehlende Angaben vorhanden sind), ist der Prozentsatz von 15 Problemen an allen von SET-Patienten angegebenen Problemen zu t5 höher, als der Prozentsatz von 16 Problemaussagen verhaltenstherapeutisch behandelter Patienten an allen zu t5 genannten Problemen in der VT-

Gruppe.

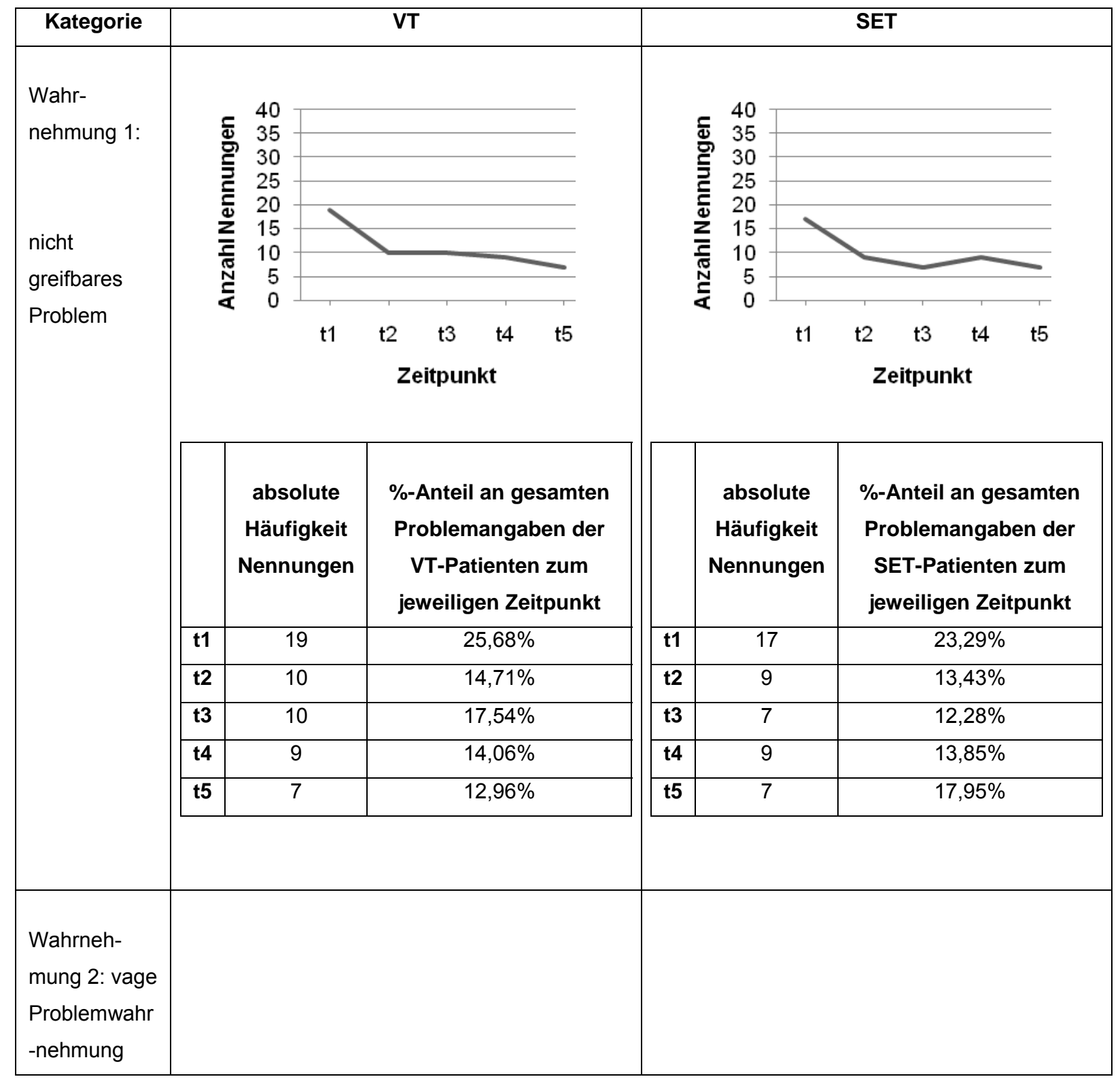




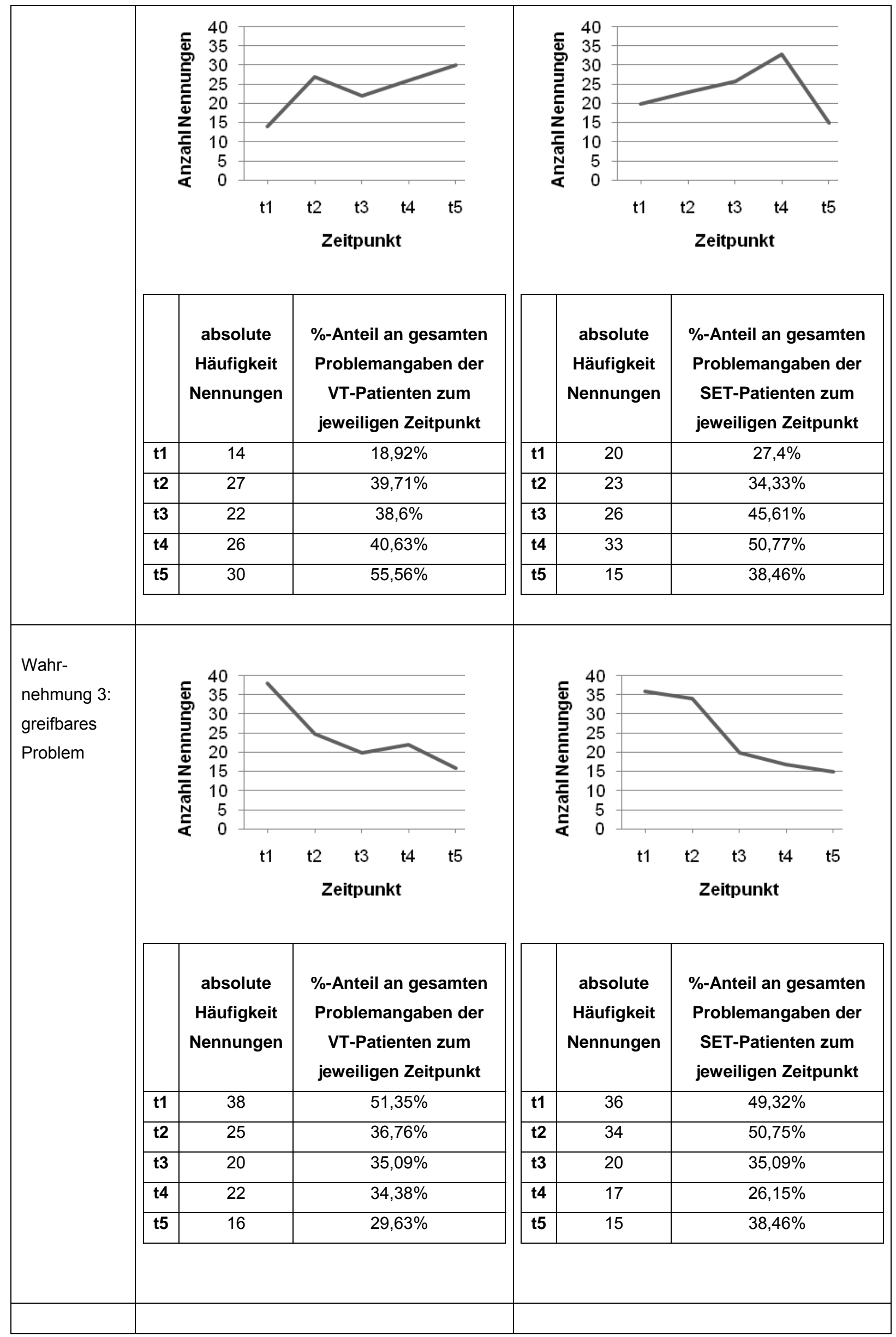




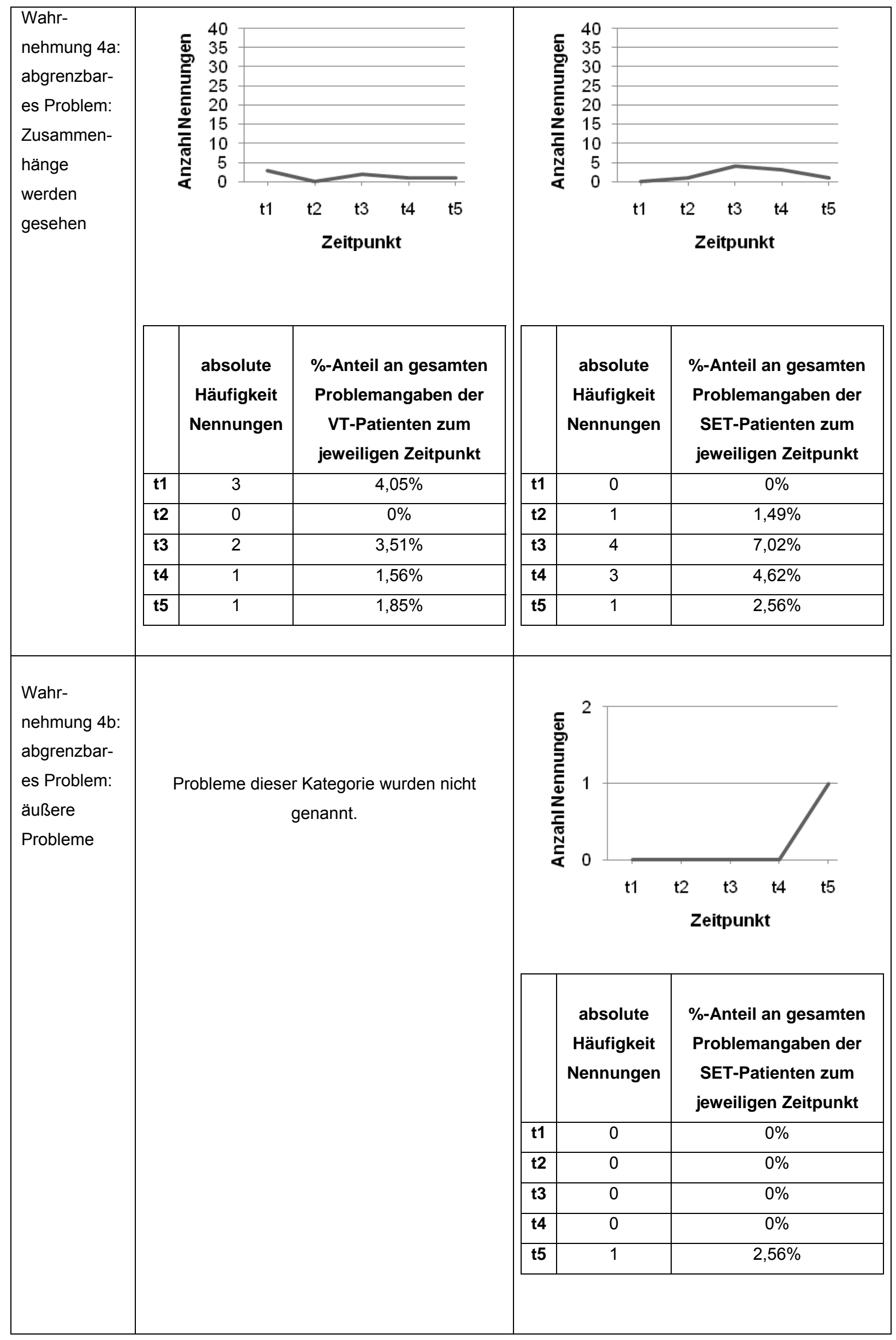


Abbildung 43: Mastery-Awareness-Scale, zeitlicher Verlauf der Problembewältigung, Vergleich der beiden Therapieformen VT und SET 


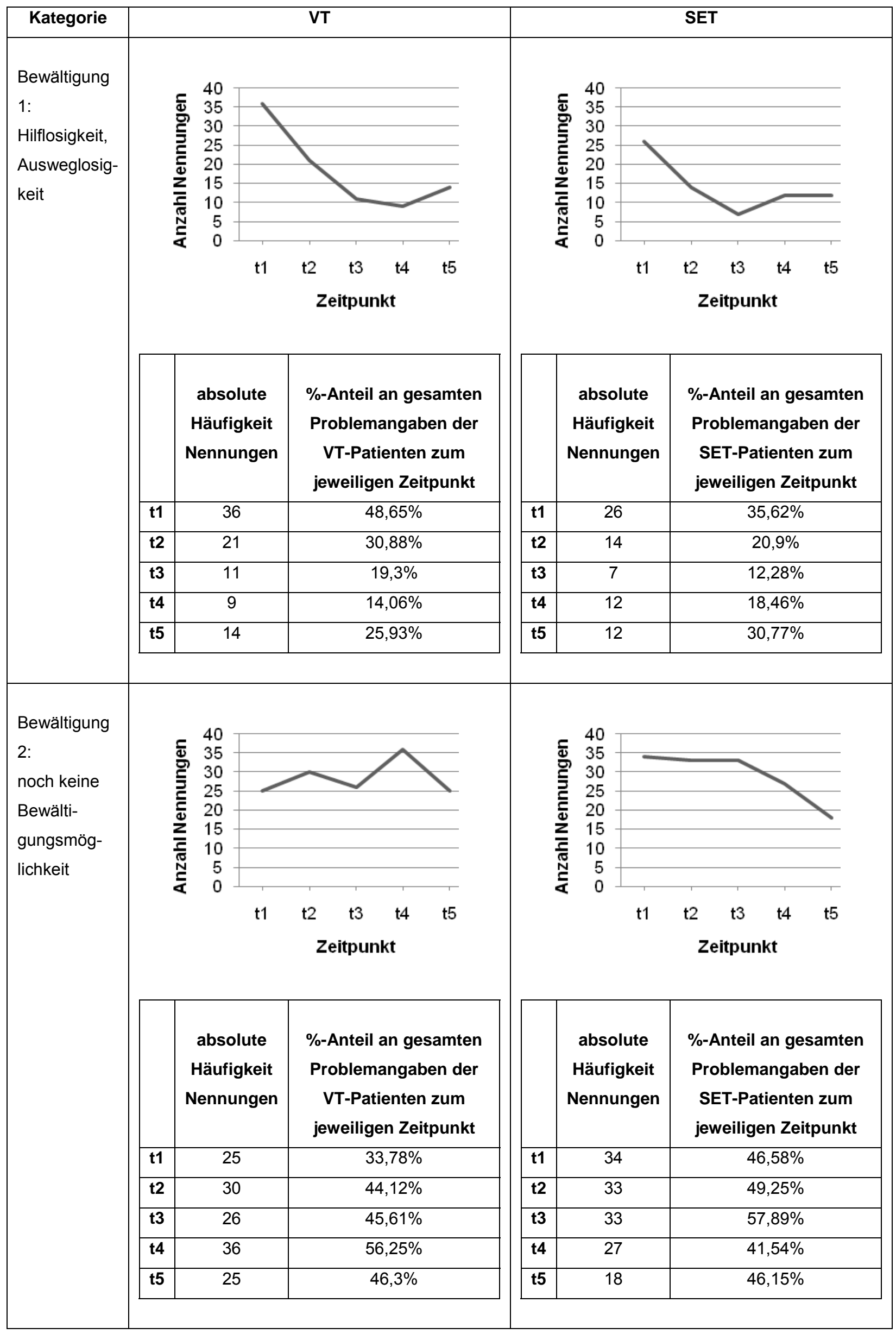




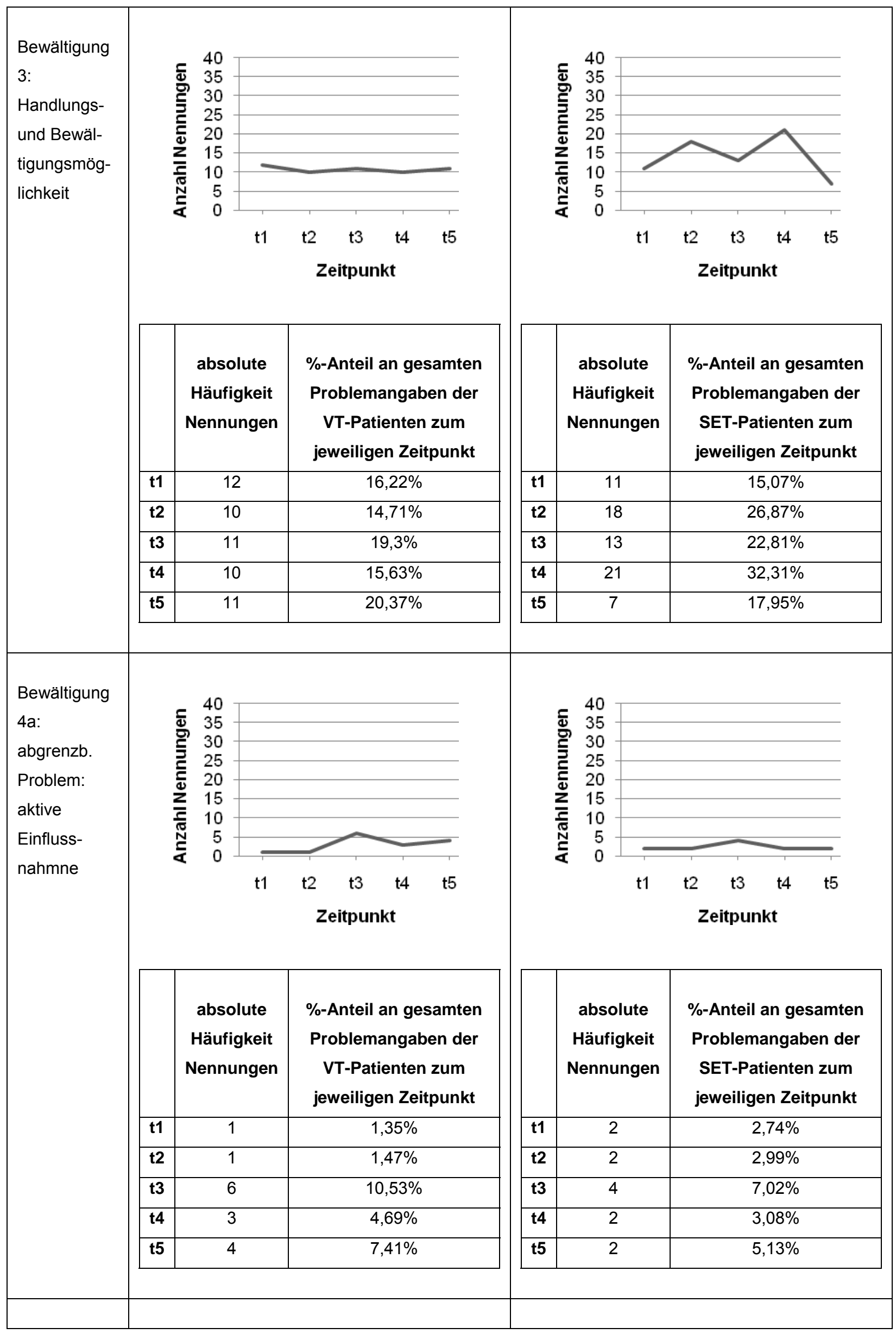




\begin{tabular}{|l|c|c|}
\hline Bewältigung & Probleme dieser Kategorie wurden nicht & Probleme dieser Kategorie wurden nicht \\
4b: abgrenz- & genannt. & genannt. \\
bares & & \\
Problem: & & \\
selbstver- & & \\
ständlicher & & \\
Umgang mit & & \\
Problem & & \\
\hline
\end{tabular}

\subsubsection{Systematische Veränderungen der Selbsteinschätzung von Patientenl Signifikanztests}

Begriffsdefinitionen der statistischen Größen:

- Median (Zentralwert):

In einer Reihe nach ihrer Größe sortierter Messwerte entspricht der Wert in der Mitte dem Median. Bei einer geraden Anzahl von Messwerten entspricht dieser Wert dem arithmetischen Mittel beider mittlerer Werte dieser Sortierung.

Dieser Wert teilt folglich eine Wertverteilung in zwei identisch große Hälften. Vorteilhaft gegenüber dem arithmetischen Mittel ist die geringere Störanfälligkeit des Medians gegenüber extremen Werten (Häcker und Stapf 2004).

- arithmetisches Mittel (in vorliegender Dissertation auch als Mittelwert bezeichnet):

Dies ist der Durchschnittswert, welcher berechnet wird, indem die Summe der Messgrößen durch ihre Anzahl dividiert wird.

- $\quad p-$ Wert ( $p$ steht für probability = Wahrscheinlichkeit):

Hierbei handelt es sich um einen Kennwert der Signifikanz bei der Anwendung statistischer Tests. Er sagt aus, wie groß die Wahrscheinlichkeit ist, dass ein ermittelter Unterschied zufällig ist (Weiß 2007). Als Testniveau (Ablehnbereich) werden in der vorliegenden Arbeit folgende Werte verwendet: $<0,05=$ signifikant, $\geq 0,05$ = nicht signifikant. Um eine zu hohe Anzahl falsch positiver Testentscheidungen zu vermeiden, wurden die p-Werte gemäß Bonferroni adjustiert (siehe 2.2.3). 


\subsection{Tabellen zu Hypothese H2.1 („Es gibt Prädiktoren, die für einen guten oder eher mäßigen Therapieverlauf sprechen.")}

Tabelle 25: Gruppeneinteilung der Patienten nach Senkung des

Leidesdruckes (unter zu t1 formulierten Problemen) zwischen $\mathrm{t} 1$ und $\mathrm{t} 3$

Gruppenzuordnung von Patienten anhand des Rückgangs im Leidensdruck von t1 zu t3 unter den zu t1 formulierten Problemen

(36 Patienten konnten in Gruppen eingeteilt und verglichen werden, da sie zu t1 und $\mathrm{t} 3$ Angaben zum Leidensdruck machten. Bei den Kategorien ist zu beachten, dass jeder Patient 3 Probleme und Ziele zu t1 angeben konnte, weshalb sich der Prozentsatz auf die angegebenen Probleme oder Ziele der jeweiligen

Gruppe zu t1 bezieht (also Anzahl Gruppenmitglieder x 3 abzüglich der fehlenden Angaben))

\begin{tabular}{|c|c|c|c|c}
\hline & Gruppe 1 & Gruppe 2 \\
$=$ guter & mittlerer & mäßiger \\
Erfolg & Erfolg & Erfolg
\end{tabular}

Gruppenzusammensetzung:

\begin{tabular}{|c|c|c|c|}
\hline Anzahl Patienten pro Gruppe & 12 & 11 & 13 \\
\hline $\begin{array}{l}\text { Anzahl in Prozent der } 36 \text { Patienten, deren Leidensdruckrückgang von } \\
\text { t1 zu t3 untersucht werden konnte }\end{array}$ & $33,33 \%$ & $30,56 \%$ & $36,11 \%$ \\
\hline $\begin{array}{l}\text { Anteil verhaltenstherapeutisch behandelter Patienten an jeweili- } \\
\text { ger Gruppe (absolute Häufigkeit und Prozentsatz der jeweiligen } \\
\text { Gruppe) }\end{array}$ & $\begin{array}{c}10 \\
(83,33 \%)\end{array}$ & $\begin{array}{c}5 \\
(45,45 \%)\end{array}$ & $\begin{array}{c}4 \\
(30,77 \%)\end{array}$ \\
\hline $\begin{array}{l}\text { Anteil nach SET behandelter Patienten an jeweiliger Gruppe (ab- } \\
\text { solute Häufigkeit und Prozentsatz der jeweiligen Gruppe) }\end{array}$ & $\begin{array}{c}2 \\
(16,67 \%)\end{array}$ & $\begin{array}{c}6 \\
(54,55 \%)\end{array}$ & $\begin{array}{c}9 \\
(69,23 \%)\end{array}$ \\
\hline $\begin{array}{l}\text { Anteil Frauen an jeweiliger Gruppe (absolute Häufigkeit und Pro- } \\
\text { zentsatz der jeweiligen Gruppe) }\end{array}$ & $\begin{array}{c}10 \\
(83,33 \%)\end{array}$ & $\begin{array}{c}9 \\
(81,82 \%)\end{array}$ & $\begin{array}{c}9 \\
(69,23 \%)\end{array}$ \\
\hline $\begin{array}{l}\text { Anteil Männer an jeweiliger Gruppe (absolute Häufigkeit und Pro- } \\
\text { zentsatz der jeweiligen Gruppe) }\end{array}$ & $\begin{array}{c}2 \\
(16,67 \%)\end{array}$ & $\begin{array}{c}2 \\
(18,18 \%)\end{array}$ & $\begin{array}{c}4 \\
(30,77 \%)\end{array}$ \\
\hline Median Geburtsjahrgang der jeweiligen Gruppe & 1964 & 1959 & 1952 \\
\hline $\begin{array}{l}\text { in fester Partnerschaft lebend (absolute Häufigkeit und Prozentsatz } \\
\text { der jeweiligen Gruppe) }\end{array}$ & $\begin{array}{c}10 \\
(83,33 \%)\end{array}$ & $\begin{array}{c}10 \\
(90,91 \%)\end{array}$ & $\begin{array}{c}11 \\
(84,62 \%)\end{array}$ \\
\hline $\begin{array}{l}\text { Median der einzelnen Mediane der Patienten einer Gruppe bezüglich } \\
\text { des Leidensdrucks der zu t1 formulierten Probleme zum Zeitpunkt t1 }\end{array}$ & 3,5 & 3 & 3 \\
\hline \multicolumn{4}{|l|}{ Kategoriensystem: } \\
\hline $\begin{array}{l}\text { körperliche Beschwerden zu t1 als Problem angegeben (Katego- } \\
\text { riensystem P1, absolute Häufigkeit der Nennungen und Prozentsatz } \\
\text { der Problemangaben der jeweiligen Gruppe) }\end{array}$ & $\begin{array}{c}3 \\
(9,38 \%)\end{array}$ & $\begin{array}{c}3 \\
(9,09 \%)\end{array}$ & $\begin{array}{c}7 \\
(18,42 \%)\end{array}$ \\
\hline
\end{tabular}




\begin{tabular}{|c|c|c|c|}
\hline $\begin{array}{l}\text { depressive Beschwerden zu t1 als Problem angegeben (Katego- } \\
\text { riensystem P2, absolute Häufigkeit der Nennungen und Prozentsatz } \\
\text { der Problemangaben der jeweiligen Gruppe) }\end{array}$ & $\begin{array}{c}2 \\
(6,25 \%)\end{array}$ & $\begin{array}{c}7 \\
(21,21 \%)\end{array}$ & $\begin{array}{c}3 \\
(7,89 \%)\end{array}$ \\
\hline $\begin{array}{l}\text { Angst zu t1 als Problem angegeben (Kategoriensystem P3, absolute } \\
\text { Häufigkeit der Nennungen und Prozentsatz der Problemangaben der } \\
\text { jeweiligen Gruppe) }\end{array}$ & $\begin{array}{c}14 \\
(43,75 \%)\end{array}$ & $\begin{array}{c}10 \\
(30,3 \%)\end{array}$ & $\begin{array}{c}9 \\
(23,68 \%)\end{array}$ \\
\hline $\begin{array}{l}\text { Sucht/ Essstörung zu t1 als Problem angegeben (Kategoriensystem } \\
\text { P4) }\end{array}$ & 0 & 0 & 0 \\
\hline $\begin{array}{l}\text { mangelndes Selbstwertgefühl/ Selbstvertrauen zu t1 als Problem } \\
\text { angegeben (Kategoriensystem P5, absolute Häufigkeit der Nennun- } \\
\text { gen und Prozentsatz der Problemangaben der jeweiligen Gruppe) }\end{array}$ & $\begin{array}{c}2 \\
(6,25 \%)\end{array}$ & 0 & $\begin{array}{c}1 \\
(2,63 \%)\end{array}$ \\
\hline $\begin{array}{l}\text { ungelöste Problemel Konflikte (z.B. Alltag und Beruf) zu t1 ange- } \\
\text { geben (Kategoriensystem P6, absolute Häufigkeit der Nennungen } \\
\text { und Prozentsatz der Problemangaben der jeweiligen Gruppe) }\end{array}$ & $\begin{array}{c}7 \\
(21,88 \%)\end{array}$ & $\begin{array}{c}6 \\
(18,18 \%)\end{array}$ & $\begin{array}{c}11 \\
(28,95 \%)\end{array}$ \\
\hline $\begin{array}{l}\text { sonstige selbstbezogene Probleme zu t1 angegeben (Kategorien- } \\
\text { system P7, absolute Häufigkeit der Nennungen und Prozentsatz der } \\
\text { Problemangaben der jeweiligen Gruppe) }\end{array}$ & $\begin{array}{c}2 \\
(6,25 \%)\end{array}$ & $\begin{array}{c}2 \\
(6,06 \%)\end{array}$ & $\begin{array}{c}1 \\
(2,63 \%)\end{array}$ \\
\hline $\begin{array}{l}\text { interpersonelle Probleme zu t1 angegeben (Kategoriensystem P8, } \\
\text { Sub-Kategorien summiert, absolute Häufigkeit der Nennungen und } \\
\text { Prozentsatz der Problemangaben der jeweiligen Gruppe) }\end{array}$ & $\begin{array}{c}2 \\
(6,25 \%)\end{array}$ & $\begin{array}{c}4 \\
(12,12 \%)\end{array}$ & $\begin{array}{c}3 \\
(7,89 \%)\end{array}$ \\
\hline $\begin{array}{l}\text { existentielle Probleme zu t1 angegeben (Kategoriensystem P9, } \\
\text { absolute Häufigkeit der Nennungen und Prozentsatz der Probleman- } \\
\text { gaben der jeweiligen Gruppe) }\end{array}$ & 0 & $\begin{array}{c}1 \\
(3,03 \%)\end{array}$ & 0 \\
\hline $\begin{array}{l}\text { Zwänge als Problem zu t1 angegeben (Kategoriensystem P10, abso- } \\
\text { lute Häufigkeit der Nennungen und Prozentsatz der Problemangaben } \\
\text { der jeweiligen Gruppe) }\end{array}$ & 0 & 0 & $\begin{array}{c}3 \\
(7,89 \%)\end{array}$ \\
\hline $\begin{array}{l}\text { einzelne fehlende Problemangaben zu t1 (absolute Häufigkeit und } \\
\text { Prozentsatz der möglichen Problemangaben der jeweiligen Gruppe) }\end{array}$ & $\begin{array}{c}4 \\
(11,11 \%)\end{array}$ & 0 & $\begin{array}{c}1 \\
(2,63 \%)\end{array}$ \\
\hline $\begin{array}{l}\text { körperliche Beschwerden zu bessern zu t1 als Ziel angegeben } \\
\text { (Kategoriensystem Z1, absolute Häufigkeit der Nennungen und Pro- } \\
\text { zentsatz der Zielangaben der jeweiligen Gruppe) }\end{array}$ & $\begin{array}{c}4 \\
(12,5 \%)\end{array}$ & $\begin{array}{c}3 \\
(9,09 \%)\end{array}$ & $\begin{array}{c}3 \\
(7,89 \%)\end{array}$ \\
\hline $\begin{array}{l}\text { depressive Beschwerden zu bessern zu t1 als Ziel angegeben } \\
\text { (Kategoriensystem Z2, absolute Häufigkeit der Nennungen und Pro- } \\
\text { zentsatz der Zielangaben der jeweiligen Gruppe) }\end{array}$ & 0 & 0 & $\begin{array}{c}1 \\
(2,63 \%)\end{array}$ \\
\hline $\begin{array}{l}\text { Angst zu bewältigen zu t1 als Ziel angegeben (Kategoriensystem } \\
\text { Z3, absolute Häufigkeit der Nennungen und Prozentsatz der Zielan- } \\
\text { gaben der jeweiligen Gruppe) }\end{array}$ & $\begin{array}{c}10 \\
(31,25 \%)\end{array}$ & $\begin{array}{c}9 \\
(27,27 \%)\end{array}$ & $\begin{array}{c}8 \\
(21,05 \%)\end{array}$ \\
\hline $\begin{array}{l}\text { Sucht/ Essstörung zu bewältigen zu t1 als Ziel angegeben (Kate- } \\
\text { goriensystem Z4) }\end{array}$ & 0 & 0 & 0 \\
\hline $\begin{array}{l}\text { Selbstvertrauen zu stärken zu t1 als Ziel angegeben (Kategorien- } \\
\text { system Z5, absolute Häufigkeit der Nennungen und Prozentsatz der } \\
\text { Zielangaben der jeweiligen Gruppe) }\end{array}$ & $\begin{array}{c}3 \\
(9,38 \%)\end{array}$ & $\begin{array}{c}1 \\
(3,03 \%)\end{array}$ & $\begin{array}{c}2 \\
(5,26 \%)\end{array}$ \\
\hline $\begin{array}{l}\text { äußere Probleme zu bewältigen (z.B. Beruf, Finanzen, Alltag) zu t1 } \\
\text { als Ziel angegeben (Kategoriensystem Z6, absolute Häufigkeit der }\end{array}$ & $\begin{array}{c}1 \\
(3,13 \%)\end{array}$ & $\begin{array}{c}1 \\
(3,03 \%)\end{array}$ & $\begin{array}{c}3 \\
(7,89 \%)\end{array}$ \\
\hline
\end{tabular}




\begin{tabular}{|c|c|c|c|}
\hline \multicolumn{4}{|l|}{ Nennungen und Prozentsatz der Zielangaben der jeweiligen Gruppe) } \\
\hline $\begin{array}{l}\text { Ruhel Gelassenheit zu finden zu t1 als Ziel angegeben (Katego- } \\
\text { riensystem Z7, absolute Häufigkeit der Nennungen und Prozentsatz } \\
\text { der Zielangaben der jeweiligen Gruppe) }\end{array}$ & $\begin{array}{c}8 \\
(25 \%)\end{array}$ & $\begin{array}{c}14 \\
(42,42 \%)\end{array}$ & $\begin{array}{c}15 \\
(39,47 \%)\end{array}$ \\
\hline $\begin{array}{l}\text { interpersonelle Probleme zu lösen zu t1 als Ziel angegeben (Kate- } \\
\text { goriensystem Z8, Sub-Kategorien summiert, absolute Häufigkeit der } \\
\text { Nennungen und Prozentsatz der Zielangaben der jeweiligen Gruppe) }\end{array}$ & $\begin{array}{c}4 \\
(12,5 \%)\end{array}$ & $\begin{array}{c}2 \\
(6,06 \%)\end{array}$ & $\begin{array}{c}2 \\
(5,26 \%)\end{array}$ \\
\hline $\begin{array}{l}\text { Freiheiten/ Fähigkeiten zu erlangen zu t1 als Ziel angegeben (Kate- } \\
\text { goriensystem Z9, absolute Häufigkeit der Nennungen und Prozent- } \\
\text { satz der Zielangaben der jeweiligen Gruppe) }\end{array}$ & $\begin{array}{c}2 \\
(6,25 \%)\end{array}$ & $\begin{array}{c}3 \\
(9,09 \%)\end{array}$ & $\begin{array}{c}3 \\
(7,89 \%)\end{array}$ \\
\hline $\begin{array}{l}\text { Zwänge abzubauen zu t1 als Ziel angegeben (Kategoriensystem } \\
\text { Z10, absolute Häufigkeit der Nennungen und Prozentsatz der Zielan- } \\
\text { gaben der jeweiligen Gruppe) }\end{array}$ & 0 & 0 & $\begin{array}{c}1 \\
(2,63 \%)\end{array}$ \\
\hline $\begin{array}{l}\text { einzelne fehlende Zielangaben zu t1 (absolute Häufigkeit und Pro- } \\
\text { zentsatz der möglichen Zielangaben der jeweiligen Gruppe) }\end{array}$ & $\begin{array}{c}4 \\
(11,11 \%)\end{array}$ & 0 & $\begin{array}{c}1 \\
(2,56 \%)\end{array}$ \\
\hline \multicolumn{4}{|l|}{ Mastery-Awareness-Scale: } \\
\hline $\begin{array}{l}\text { Problem wird als nicht greifbar wahrgenommen (Mastery- } \\
\text { Awareness-Scale W1, absolute Häufigkeit der Nennungen und Pro- } \\
\text { zentsatz der Problemangaben der jeweiligen Gruppe) }\end{array}$ & $\begin{array}{c}3 \\
(9,38 \%)\end{array}$ & $\begin{array}{c}10 \\
(30,3 \%)\end{array}$ & $\begin{array}{c}10 \\
(26,32 \%)\end{array}$ \\
\hline $\begin{array}{l}\text { vage Problemwahrnehmung (Mastery-Awareness-Scale W2, abso- } \\
\text { lute Häufigkeit der Nennungen und Prozentsatz der Problemangaben } \\
\text { der jeweiligen Gruppe) }\end{array}$ & $\begin{array}{c}4 \\
(12,5 \%)\end{array}$ & $\begin{array}{c}5 \\
(15,15 \%)\end{array}$ & $\begin{array}{c}16 \\
(42,11 \%)\end{array}$ \\
\hline $\begin{array}{l}\text { Problem wird als greifbar wahrgenommen (Mastery-Awareness- } \\
\text { Scale W3, absolute Häufigkeit der Nennungen und Prozentsatz der } \\
\text { Problemangaben der jeweiligen Gruppe) }\end{array}$ & $\begin{array}{c}24 \\
(75 \%)\end{array}$ & $\begin{array}{c}17 \\
(51,52 \%)\end{array}$ & $\begin{array}{c}12 \\
(31,58 \%)\end{array}$ \\
\hline $\begin{array}{l}\text { Problem wird als abgrenzbar wahrgenommen, Zusammenhänge } \\
\text { werden gesehen (Mastery-Awareness-Scale W4a, absolute Häufig- } \\
\text { keit der Nennungen und Prozentsatz der Problemangaben der jewei- } \\
\text { ligen Gruppe) }\end{array}$ & $\begin{array}{c}1 \\
(3,13 \%)\end{array}$ & $\begin{array}{c}1 \\
(3,03 \%)\end{array}$ & 0 \\
\hline $\begin{array}{l}\text { Problem wird als abgrenzbar wahrgenommen, äußere Probleme } \\
\text { (Mastery-Awareness-Scale W4b) }\end{array}$ & 0 & 0 & 0 \\
\hline $\begin{array}{l}\text { einzelne fehlende Problemangaben zu t1 (absolute Häufigkeit und } \\
\text { Prozentsatz der möglichen Problemangaben der jeweiligen Gruppe) }\end{array}$ & $\begin{array}{c}4 \\
(11,11 \%)\end{array}$ & 0 & $\begin{array}{c}1 \\
(2,63 \%)\end{array}$ \\
\hline $\begin{array}{l}\text { Hilflosigkeit, Ausweglosigkeit im Umgang mit dem Problem } \\
\text { (Mastery-Awareness-Scale M1, absolute Häufigkeit der Nennungen } \\
\text { und Prozentsatz der Problemangaben der jeweiligen Gruppe) }\end{array}$ & $\begin{array}{c}10 \\
(31,25 \%)\end{array}$ & $\begin{array}{c}21 \\
(63,64 \%)\end{array}$ & $\begin{array}{c}15 \\
(39,47 \%)\end{array}$ \\
\hline $\begin{array}{l}\text { noch keine Bewältigungsmöglichkeit des Problems (Mastery- } \\
\text { Awareness-Scale M2, absolute Häufigkeit der Nennungen und Pro- } \\
\text { zentsatz der Problemangaben der jeweiligen Gruppe) }\end{array}$ & $\begin{array}{c}12 \\
(37,5 \%)\end{array}$ & $\begin{array}{c}6 \\
(18,18 \%)\end{array}$ & $\begin{array}{c}19 \\
(50 \%)\end{array}$ \\
\hline $\begin{array}{l}\text { Handlungs- und Bewältigungsmöglichkeit im Umgang mit dem } \\
\text { Problem (Mastery-Awareness-Scale M3, absolute Häufigkeit der } \\
\text { Nennungen und Prozentsatz der Problemangaben der jeweiligen }\end{array}$ & $\begin{array}{c}8 \\
(25 \%)\end{array}$ & $\begin{array}{c}5 \\
(15,15 \%)\end{array}$ & $\begin{array}{c}4 \\
(10,53 \%)\end{array}$ \\
\hline
\end{tabular}




\begin{tabular}{|c|c|c|c|}
\hline Gruppe) & & & \\
\hline $\begin{array}{l}\text { aktive Einflussnahme bei Bewältigung des Problems (Mastery- } \\
\text { Awareness-Scale M4a, absolute Häufigkeit der Nennungen und Pro- } \\
\text { zentsatz der Problemangaben der jeweiligen Gruppe) }\end{array}$ & $\begin{array}{c}2 \\
(6,25 \%)\end{array}$ & $\begin{array}{c}1 \\
(3,03 \%)\end{array}$ & 0 \\
\hline $\begin{array}{l}\text { selbstverständlicher Umgang mit dem Problem (Mastery- } \\
\text { Awareness-Scale M4b) }\end{array}$ & 0 & 0 & 0 \\
\hline $\begin{array}{l}\text { einzelne fehlende Problemangaben zu t1 (absolute Häufigkeit und } \\
\text { Prozentsatz der möglichen Problemangaben der jeweiligen Gruppe) }\end{array}$ & $\begin{array}{c}4 \\
(11,11 \%)\end{array}$ & 0 & $\begin{array}{c}1 \\
(2,63 \%)\end{array}$ \\
\hline
\end{tabular}

\subsection{Tabellen zu Hypothese H2.2 („Die in Hypothese H2.1 ermittelten Prä- diktoren für den Erfolg von Therapieverläufen sind innerhalb der beiden untersuchten Therapieverfahren (supportiv-expressive Therapie (SET) und Verhaltenstherapie) unterschiedlich verteilt.")}

Tabelle 26: Sub-Gruppen-Einteilung der Patienten einer Therapierichtung (VT, SET) nach Senkung des Leidesdruckes (unter zu t1 formulierten Problemen) zwischen t1 und t3

Gruppenzuordnung von Patienten anhand des Leidensdruck-Rückgangs von t1 zu t3 unter den zu t1 formulierten Problemen

(36 Patienten konnten in Gruppen eingeteilt und verglichen werden, da sie zu t1 und t3 Angaben zum Leidensdruck machten. Bei den Kategorien ist zu beachten, dass jeder Patient 3 Probleme und Ziele zu t1 angeben konnte, weshalb sich der Prozentsatz auf die angegebenen Probleme oder Ziele der jeweiligen

Sub-Gruppe zu t1 bezieht (also Anzahl Gruppenmitglieder x 3 abzüglich der fehlenden Angaben))

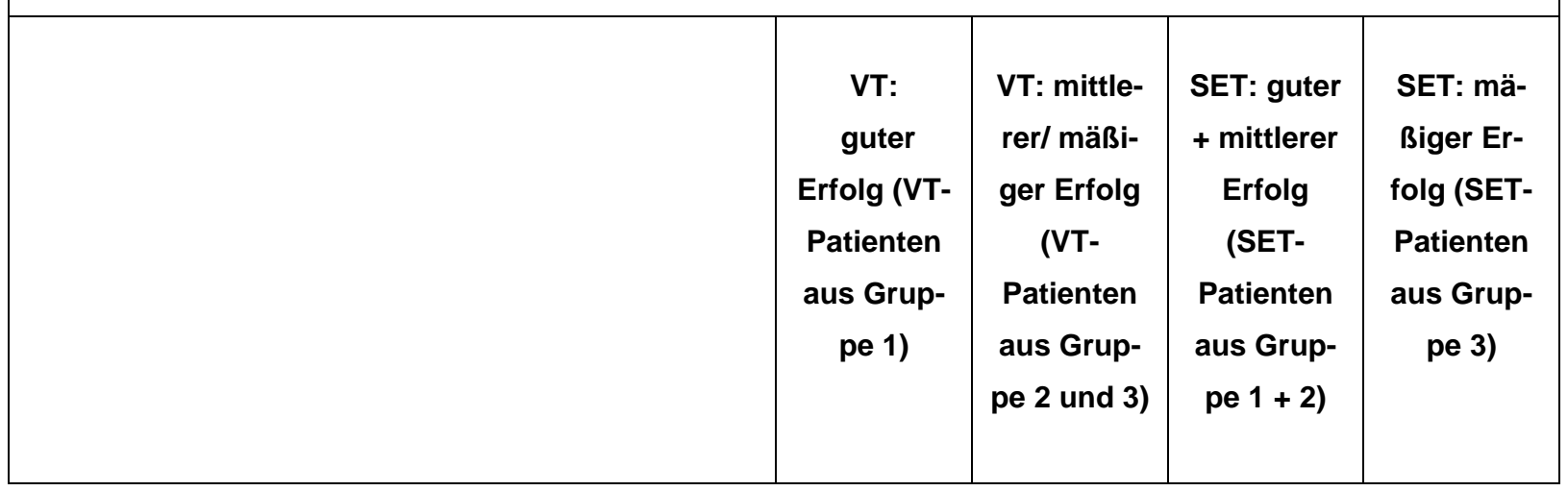




\section{Gruppenzusammensetzung:}

\begin{tabular}{|c|c|c|c|c|}
\hline Anzahl Patienten pro Sub-Gruppe & 10 & 9 & 8 & 9 \\
\hline $\begin{array}{l}\text { Anzahl in Prozent der Patienten einer Therapiegruppe } \\
\text { (VT } 19 \text { Patienten, SET } 17 \text { Patienten), deren Leidens- } \\
\text { druckrückgang von t1 zu t3 untersucht werden konnte }\end{array}$ & $52,63 \%$ & $47,37 \%$ & $47,06 \%$ & $52,94 \%$ \\
\hline $\begin{array}{l}\text { Anteil Frauen an jeweiliger Sub-Gruppe (absolute } \\
\text { Häufigkeit und Prozentsatz der jeweiligen Subgruppe) }\end{array}$ & $\begin{array}{c}8 \\
(80 \%)\end{array}$ & $\begin{array}{c}6 \\
(66,67 \%)\end{array}$ & $\begin{array}{c}7 \\
(87,5 \%)\end{array}$ & $\begin{array}{c}7 \\
(77,78 \%)\end{array}$ \\
\hline $\begin{array}{l}\text { Anteil Männer an jeweiliger Sub-Gruppe (absolute } \\
\text { Häufigkeit und Prozentsatz der jeweiligen Sub- } \\
\text { Gruppe) }\end{array}$ & $\begin{array}{c}2 \\
(20 \%)\end{array}$ & $\begin{array}{c}3 \\
(33,33 \%)\end{array}$ & $\begin{array}{c}1 \\
(12,5 \%)\end{array}$ & $\begin{array}{c}2 \\
(28,57 \%)\end{array}$ \\
\hline Median Geburtsjahrgang der jeweiligen Sub-Gruppe & 1964 & 1951 & 1963 & 1952 \\
\hline $\begin{array}{l}\text { in fester Partnerschaft lebend (absolute Häufigkeit } \\
\text { und Prozentsatz der jeweiligen Sub-Gruppe) }\end{array}$ & $\begin{array}{c}8 \\
(80 \%)\end{array}$ & $\begin{array}{c}6 \\
(66,67 \%)\end{array}$ & $\begin{array}{c}8 \\
(100 \%)\end{array}$ & $\begin{array}{c}9 \\
(100 \%)\end{array}$ \\
\hline $\begin{array}{l}\text { Median der einzelnen Mediane der Patienten einer } \\
\text { Sub-Gruppe bezüglich des Leidensdrucks der zu t1 } \\
\text { formulierten Probleme zum Zeitpunkt t1 }\end{array}$ & 4 & 3 & 3 & 3 \\
\hline
\end{tabular}

\section{Kategoriensystem:}

\begin{tabular}{|c|c|c|c|c|}
\hline $\begin{array}{l}\text { körperliche Beschwerden zu t1 als Problem ange- } \\
\text { geben (Kategoriensystem P1, absolute Häufigkeit der } \\
\text { Nennungen und Prozentsatz der Problemangaben der } \\
\text { jeweiligen Sub-Gruppe) }\end{array}$ & $\begin{array}{c}2 \\
(7,69 \%)\end{array}$ & $\begin{array}{c}2 \\
(7,41 \%)\end{array}$ & $\begin{array}{c}3 \\
(12,5 \%)\end{array}$ & $\begin{array}{c}6 \\
(23,08 \%)\end{array}$ \\
\hline $\begin{array}{l}\text { depressive Beschwerden zu t1 als Problem angege- } \\
\text { ben (Kategoriensystem P2, absolute Häufigkeit der } \\
\text { Nennungen und Prozentsatz der Problemangaben der } \\
\text { jeweiligen Sub-Gruppe) }\end{array}$ & $\begin{array}{c}2 \\
(7,69 \%)\end{array}$ & $\begin{array}{c}3 \\
(11,11 \%)\end{array}$ & $\begin{array}{c}5 \\
(20,83 \%)\end{array}$ & $\begin{array}{c}2 \\
(7,69 \%)\end{array}$ \\
\hline $\begin{array}{l}\text { Angst zu t1 als Problem angegeben (Kategoriensys- } \\
\text { tem P3, absolute Häufigkeit der Nennungen und Pro- } \\
\text { zentsatz der Problemangaben der jeweiligen Sub- } \\
\text { Gruppe) }\end{array}$ & $\begin{array}{c}13 \\
(50 \%)\end{array}$ & $\begin{array}{c}8 \\
(29,63 \%)\end{array}$ & $\begin{array}{c}8 \\
(33,33 \%)\end{array}$ & $\begin{array}{c}4 \\
(15,38 \%)\end{array}$ \\
\hline $\begin{array}{l}\text { Sucht/ Essstörung zu t1 als Problem angegeben } \\
\text { (Kategoriensystem P4) }\end{array}$ & 0 & 0 & 0 & 0 \\
\hline $\begin{array}{l}\text { mangelndes Selbstwertgefühl/ Selbstvertrauen zu } \\
\text { t1 als Problem angegeben (Kategoriensystem P5, } \\
\text { absolute Häufigkeit der Nennungen und Prozentsatz } \\
\text { der Problemangaben der jeweiligen Sub-Gruppe) }\end{array}$ & $\begin{array}{c}2 \\
(7,69 \%)\end{array}$ & $\begin{array}{c}1 \\
(3,7 \%)\end{array}$ & 0 & 0 \\
\hline $\begin{array}{l}\text { ungelöste Problemel Konflikte (z.B. Alltag und } \\
\text { Beruf) zu t1 angegeben (Kategoriensystem P6, abso- } \\
\text { lute Häufigkeit der Nennungen und Prozentsatz der } \\
\text { Problemangaben der jeweiligen Sub-Gruppe) }\end{array}$ & $\begin{array}{c}4 \\
(15,38 \%)\end{array}$ & $\begin{array}{c}4 \\
(14,81 \%)\end{array}$ & $\begin{array}{c}7 \\
(29,17 \%)\end{array}$ & $\begin{array}{c}9 \\
(34,62 \%)\end{array}$ \\
\hline $\begin{array}{l}\text { sonstige selbstbezogene Probleme zu t1 angege- } \\
\text { ben (Kategoriensystem P7, absolute Häufigkeit der }\end{array}$ & $\begin{array}{c}1 \\
(3,85 \%)\end{array}$ & $\begin{array}{c}2 \\
(7,41 \%)\end{array}$ & $\begin{array}{c}1 \\
(4,17 \%)\end{array}$ & $\begin{array}{c}1 \\
(3,85 \%)\end{array}$ \\
\hline
\end{tabular}




\begin{tabular}{|c|c|c|c|c|}
\hline $\begin{array}{l}\text { Nennungen und Prozentsatz der Problemangaben der } \\
\text { jeweiligen Sub-Gruppe) }\end{array}$ & & & & \\
\hline $\begin{array}{l}\text { interpersonelle Probleme zu t1 angegeben (Katego- } \\
\text { riensystem P8, Sub-Kategorien summiert, absolute } \\
\text { Häufigkeit der Nennungen und Prozentsatz der Prob- } \\
\text { lemangaben der jeweiligen Sub-Gruppe) }\end{array}$ & $\begin{array}{c}2 \\
(7,69 \%)\end{array}$ & $\begin{array}{c}5 \\
(18,52 \%)\end{array}$ & 0 & $\begin{array}{c}2 \\
(7,69 \%)\end{array}$ \\
\hline $\begin{array}{l}\text { existentielle Probleme zu t1 angegeben (Kategorien- } \\
\text { system P9, absolute Häufigkeit der Nennungen und } \\
\text { Prozentsatz der Problemangaben der jeweiligen Sub- } \\
\text { Gruppe) }\end{array}$ & 0 & $\begin{array}{c}1 \\
(3,7 \%)\end{array}$ & 0 & 0 \\
\hline $\begin{array}{l}\text { Zwänge als Problem zu t1 angegeben (Kategoriensys- } \\
\text { tem P10, absolute Häufigkeit der Nennungen und } \\
\text { Prozentsatz der Problemangaben der jeweiligen Sub- } \\
\text { Gruppe) }\end{array}$ & 0 & $\begin{array}{c}1 \\
(3,7 \%)\end{array}$ & 0 & $\begin{array}{c}2 \\
(7,69 \%)\end{array}$ \\
\hline $\begin{array}{l}\text { einzelne fehlende Problemangaben zu t1 (absolute } \\
\text { Häufigkeit und Prozentsatz der möglichen Probleman- } \\
\text { gaben der jeweiligen Sub-Gruppe) }\end{array}$ & $\begin{array}{c}4 \\
(13,33 \%)\end{array}$ & 0 & 0 & $\begin{array}{c}1 \\
(3,7 \%)\end{array}$ \\
\hline $\begin{array}{l}\text { körperliche Beschwerden zu bessern zu t1 als Ziel } \\
\text { angegeben (Kategoriensystem Z1, absolute Häufigkeit } \\
\text { der Nennungen und Prozentsatz der Zielangaben der } \\
\text { jeweiligen Sub-Gruppe) }\end{array}$ & $\begin{array}{c}3 \\
(11,54 \%)\end{array}$ & $\begin{array}{c}2 \\
(7,41 \%)\end{array}$ & $\begin{array}{c}2 \\
(8,33 \%)\end{array}$ & $\begin{array}{c}3 \\
(11,54 \%)\end{array}$ \\
\hline $\begin{array}{l}\text { depressive Beschwerden zu bessern zu t1 als Ziel } \\
\text { angegeben (Kategoriensystem Z2, absolute Häufigkeit } \\
\text { der Nennungen und Prozentsatz der Zielangaben der } \\
\text { jeweiligen Sub-Gruppe) }\end{array}$ & 0 & 0 & 0 & $\begin{array}{c}1 \\
(3,85 \%)\end{array}$ \\
\hline $\begin{array}{l}\text { Angst zu bewältigen zu t1 als Ziel angegeben (Kate- } \\
\text { goriensystem Z3, absolute Häufigkeit der Nennungen } \\
\text { und Prozentsatz der Zielangaben der jeweiligen Sub- } \\
\text { Gruppe) }\end{array}$ & $\begin{array}{c}8 \\
(30,77 \%)\end{array}$ & $\begin{array}{c}8 \\
(29,63 \%)\end{array}$ & $\begin{array}{c}7 \\
(29,17 \%)\end{array}$ & $\begin{array}{c}4 \\
(15,38 \%)\end{array}$ \\
\hline $\begin{array}{l}\text { Sucht/ Essstörung zu bewältigen zu t1 als Ziel an- } \\
\text { gegeben (Kategoriensystem Z4) }\end{array}$ & 0 & 0 & 0 & 0 \\
\hline $\begin{array}{l}\text { Selbstvertrauen zu stärken zu t1 als Ziel angegeben } \\
\text { (Kategoriensystem Z5, absolute Häufigkeit der Nen- } \\
\text { nungen und Prozentsatz der Zielangaben der jeweili- } \\
\text { gen Sub-Gruppe) }\end{array}$ & $\begin{array}{c}3 \\
(11,54 \%)\end{array}$ & $\begin{array}{c}2 \\
(7,41 \%)\end{array}$ & 0 & $\begin{array}{c}1 \\
(3,85 \%)\end{array}$ \\
\hline $\begin{array}{l}\text { äußere Probleme zu bewältigen (z.B. Beruf, Finan- } \\
\text { zen, Alltag) zu t1 als Ziel angegeben (Kategoriensys- } \\
\text { tem Z6, absolute Häufigkeit der Nennungen und Pro- } \\
\text { zentsatz der Zielangaben der jeweiligen Sub-Gruppe) }\end{array}$ & $\begin{array}{c}1 \\
(3,85 \%)\end{array}$ & 0 & $\begin{array}{c}1 \\
(4,17 \%)\end{array}$ & $\begin{array}{c}3 \\
(11,54 \%)\end{array}$ \\
\hline $\begin{array}{l}\text { Ruhe/ Gelassenheit zu finden zu t1 als Ziel angege- } \\
\text { ben (Kategoriensystem Z7, absolute Häufigkeit der } \\
\text { Nennungen und Prozentsatz der Zielangaben der } \\
\text { jeweiligen Sub-Gruppe) }\end{array}$ & $\begin{array}{c}5 \\
(19,23 \%)\end{array}$ & $\begin{array}{c}9 \\
(33,33 \%)\end{array}$ & $\begin{array}{c}12 \\
(50 \%)\end{array}$ & $\begin{array}{c}11 \\
(42,31 \%)\end{array}$ \\
\hline interpersonelle Probleme zu lösen zu t1 als Ziel & 4 & 3 & 0 & 1 \\
\hline
\end{tabular}




\begin{tabular}{|c|c|c|c|c|}
\hline $\begin{array}{l}\text { angegeben (Kategoriensystem Z8, Sub-Kategorien } \\
\text { summiert, absolute Häufigkeit der Nennungen und } \\
\text { Prozentsatz der Zielangaben der jeweiligen Sub- } \\
\text { Gruppe) }\end{array}$ & $(15,38 \%)$ & $(11,11 \%)$ & & $(3,85 \%)$ \\
\hline $\begin{array}{l}\text { Freiheiten/ Fähigkeiten zu erlangen zu t1 als Ziel } \\
\text { angegeben (Kategoriensystem Z9, absolute Häufigkeit } \\
\text { der Nennungen und Prozentsatz der Zielangaben der } \\
\text { jeweiligen Sub-Gruppe) }\end{array}$ & $\begin{array}{c}2 \\
(7,69 \%)\end{array}$ & $\begin{array}{c}3 \\
(11,11 \%)\end{array}$ & $\begin{array}{c}3 \\
(8,33 \%)\end{array}$ & $\begin{array}{c}1 \\
(3,85 \%)\end{array}$ \\
\hline $\begin{array}{l}\text { Zwänge abzubauen zu t1 als Ziel angegeben (Kate- } \\
\text { goriensystem Z10, absolute Häufigkeit der Nennungen } \\
\text { und Prozentsatz der Zielangaben der jeweiligen Sub- } \\
\text { Gruppe) }\end{array}$ & 0 & 0 & 0 & $\begin{array}{c}1 \\
(3,85 \%)\end{array}$ \\
\hline $\begin{array}{l}\text { einzelne fehlende Zielangaben zu t1 (absolute Häu- } \\
\text { figkeit und Prozentsatz der möglichen Zielangaben der } \\
\text { jeweiligen Sub-Gruppe) }\end{array}$ & $\begin{array}{c}4 \\
(13,33 \%)\end{array}$ & 0 & 0 & $\begin{array}{c}1 \\
(3,7 \%)\end{array}$ \\
\hline \multicolumn{5}{|l|}{ Mastery-Awareness-Scale: } \\
\hline $\begin{array}{l}\text { Problem wird als nicht greifbar wahrgenommen } \\
\text { (Mastery-Awareness-Scale W1, absolute Häufigkeit } \\
\text { der Nennungen und Prozentsatz der Problemangaben } \\
\text { der jeweiligen Sub-Gruppe) }\end{array}$ & $\begin{array}{c}3 \\
(11,54 \%)\end{array}$ & $\begin{array}{c}7 \\
(25,93 \%)\end{array}$ & $\begin{array}{c}6 \\
(25 \%)\end{array}$ & $\begin{array}{c}7 \\
(26,92 \%)\end{array}$ \\
\hline $\begin{array}{l}\text { vage Problemwahrnehmung (Mastery-Awareness- } \\
\text { Scale W2, absolute Häufigkeit der Nennungen und } \\
\text { Prozentsatz der Problemangaben der jeweiligen Sub- } \\
\text { Gruppe) }\end{array}$ & $\begin{array}{c}4 \\
(15,38 \%)\end{array}$ & $\begin{array}{c}9 \\
(33,33 \%)\end{array}$ & $\begin{array}{c}2 \\
(8,33 \%)\end{array}$ & $\begin{array}{c}10 \\
(38,46 \%)\end{array}$ \\
\hline $\begin{array}{l}\text { Problem wird als greifbar wahrgenommen (Maste- } \\
\text { ry-Awareness-Scale W3, absolute Häufigkeit der Nen- } \\
\text { nungen und Prozentsatz der Problemangaben der } \\
\text { jeweiligen Sub-Gruppe) }\end{array}$ & $\begin{array}{c}18 \\
(69,23 \%)\end{array}$ & $\begin{array}{c}10 \\
(37,04 \%)\end{array}$ & $\begin{array}{c}16 \\
(66,67 \%)\end{array}$ & $\begin{array}{c}9 \\
(34,62 \%)\end{array}$ \\
\hline $\begin{array}{l}\text { Problem wird als abgrenzbar wahrgenommen, } \\
\text { Zusammenhänge werden gesehen (Mastery- } \\
\text { Awareness-Scale W4a, absolute Häufigkeit der Nen- } \\
\text { nungen und Prozentsatz der Problemangaben der } \\
\text { jeweiligen Sub-Gruppe) }\end{array}$ & $\begin{array}{c}1 \\
(3,85 \%)\end{array}$ & $\begin{array}{c}1 \\
(3,7 \%)\end{array}$ & 0 & 0 \\
\hline $\begin{array}{l}\text { Problem wird als abgrenzbar wahrgenommen, } \\
\text { äußere Probleme (Mastery-Awareness-Scale W4b) }\end{array}$ & 0 & 0 & 0 & 0 \\
\hline $\begin{array}{l}\text { einzelne fehlende Problemangaben zu t1 (absolute } \\
\text { Häufigkeit und Prozentsatz der möglichen Probleman- } \\
\text { gaben der jeweiligen Sub-Gruppe) }\end{array}$ & $\begin{array}{c}4 \\
(13,33 \%)\end{array}$ & 0 & 0 & $\begin{array}{c}1 \\
(3,7 \%)\end{array}$ \\
\hline $\begin{array}{l}\text { Hilflosigkeit, Ausweglosigkeit im Umgang mit dem } \\
\text { Problem (Mastery-Awareness-Scale M1, absolute } \\
\text { Häufigkeit der Nennungen und Prozentsatz der Prob- } \\
\text { lemangaben der jeweiligen Sub-Gruppe) }\end{array}$ & $\begin{array}{c}10 \\
(38,46 \%)\end{array}$ & $\begin{array}{c}15 \\
(55,56 \%)\end{array}$ & $\begin{array}{c}12 \\
(50 \%)\end{array}$ & $\begin{array}{c}9 \\
(34,62 \%)\end{array}$ \\
\hline
\end{tabular}




\begin{tabular}{|c|c|c|c|c|}
\hline $\begin{array}{l}\text { noch keine Bewältigungsmöglichkeit des Prob- } \\
\text { lems (Mastery-Awareness-Scale M2, absolute Häufig- } \\
\text { keit der Nennungen und Prozentsatz der Probleman- } \\
\text { gaben der jeweiligen Sub-Gruppe) }\end{array}$ & $\begin{array}{c}11 \\
(42,31 \%)\end{array}$ & $\begin{array}{c}8 \\
(29,63 \%)\end{array}$ & $\begin{array}{c}4 \\
(16,67 \%)\end{array}$ & $\begin{array}{c}14 \\
(53,85 \%)\end{array}$ \\
\hline $\begin{array}{l}\text { Handlungs- und Bewältigungsmöglichkeit im Um- } \\
\text { gang mit dem Problem (Mastery-Awareness-Scale } \\
\text { M3, absolute Häufigkeit der Nennungen und Prozent- } \\
\text { satz der Problemangaben der jeweiligen Sub-Gruppe) }\end{array}$ & $\begin{array}{c}4 \\
(15,38 \%)\end{array}$ & $\begin{array}{c}4 \\
(14,81 \%)\end{array}$ & $\begin{array}{c}6 \\
(25 \%)\end{array}$ & $\begin{array}{c}3 \\
(11,54 \%)\end{array}$ \\
\hline $\begin{array}{l}\text { aktive Einflussnahme bei Bewältigung des Prob- } \\
\text { lems (Mastery-Awareness-Scale M4a, absolute Häu- } \\
\text { figkeit der Nennungen und Prozentsatz der Problem- } \\
\text { angaben der jeweiligen Sub-Gruppe) }\end{array}$ & $\begin{array}{c}1 \\
(3,85 \%)\end{array}$ & 0 & $\begin{array}{c}2 \\
(8,33 \%)\end{array}$ & 0 \\
\hline $\begin{array}{l}\text { selbstverständlicher Umgang mit dem Problem } \\
\text { (Mastery-Awareness-Scale M4b) }\end{array}$ & 0 & 0 & 0 & 0 \\
\hline $\begin{array}{l}\text { einzelne fehlende Problemangaben zu t1 (absolute } \\
\text { Häufigkeit und Prozentsatz der möglichen Probleman- } \\
\text { gaben der jeweiligen Sub-Gruppe) }\end{array}$ & $\begin{array}{c}4 \\
(13,33 \%)\end{array}$ & 0 & 0 & $\begin{array}{c}1 \\
(3,7 \%)\end{array}$ \\
\hline
\end{tabular}




\section{Danksagung}

Ich möchte mich bei allen Personen bedanken, die mir durch ihre vielfältige Unterstützung geholfen haben, diese Arbeit zu erstellen.

Mein Dank gilt den Studienteilnehmern und deren behandelnden Psychotherapeuten, ohne die die Durchführung dieser Studie nicht möglich gewesen wäre.

Herzlich danken möchte ich Prof. Dr. Hermann Staats für die Betreuung meiner Dissertation, dessen Ideen, Ratschläge und Motivation maßgeblich zur Ausführung der Arbeit beitrugen.

Ebenso spreche ich meinen besonderen Dank meiner Mutter Gisela Wiegand aus, die mir eine große Hilfe bei der formalen Fertigstellung der Arbeit war. Gleiches gilt für meinen Vater Gerd Wiegand sowie Cornelie und Michael Wiegand. Außerdem möchte ich mich sehr bei Lars Pape für die wertvollen Gespräche und seine Geduld bedanken.

Des Weiteren gilt mein Dank Verena Werling für die konstruktive und angenehme Zusammenarbeit.

Für die Unterstützung bei der statistischen Auswertung der Daten danke ich Dr. Klaus Jung sowie den wissenschaftlichen Mitarbeitern der Abteilung Medizinische Statistik der Georg-August-Universität Göttingen.

Prof. Dr. Christoph Hermann-Lingen der Abteilung Psychosomatische Medizin und Psychotherapie der Georg-August-Universität Göttingen danke ich für die wertvollen Ratschläge im Rahmen der Doktorandenkolloquien.

Für die Betreuung der Studiendaten bedanke ich mich abschließend bei Frau Dipl. Psychologin Simone Salzer der Abteilung Psychosomatische Medizin und Psychotherapie der Georg-August-Universität Göttingen. 